\title{
A Highly Active Palladium Catalyst for Intermolecular Hydroamination. Factors that Control Reactivity and Additions of Functionalized Anilines to Dienes and Vinylarenes
}

\author{
Adam M. Johns, Masaru Utsunomiya, Christopher D. Incarvito and John F. Hartwig* \\ Yale University, Department of Chemistry,P.O. Box 208107, New Haven, Connecticut 06520-8107
}

\section{Supporting Information}

\section{Table of Contents}

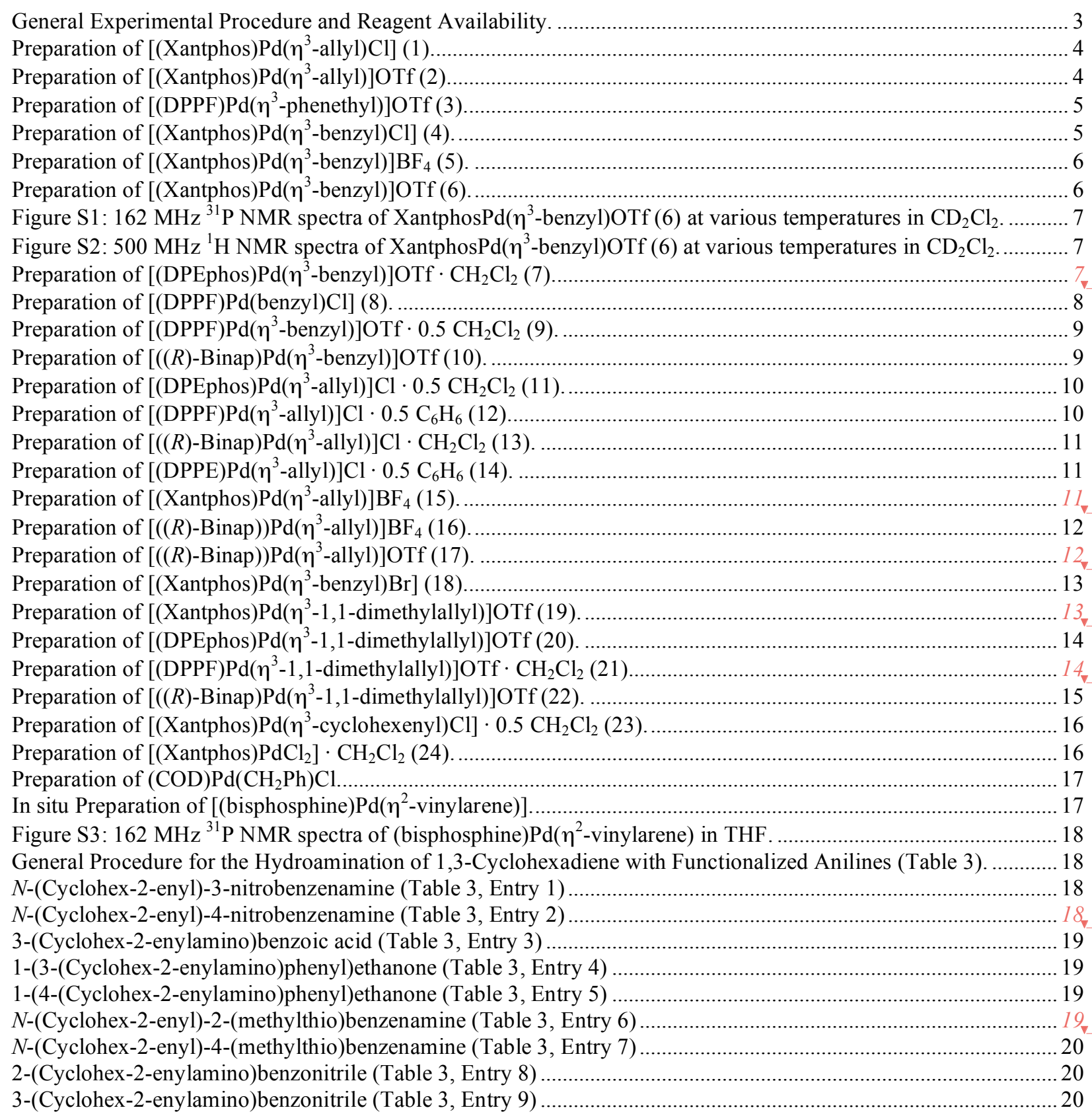




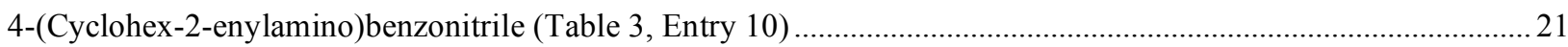

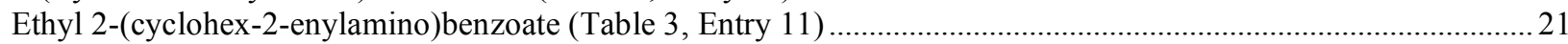

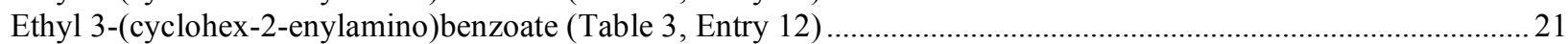

Ethyl 4-(cyclohex-2-enylamino)benzoate (Table 3, Entry 13) ................................................................................. 21

2-(Cyclohex-2-enylamino)phenol (Table 3, Entry 14) .............................................................................................22

General Procedure for the Hydroamination of Styrene with Functionalized Anilines (Table 4)................................22

2-(1-Phenylethylamino)phenol (Table 4, Entry 1) ……………..............................................................................22

| 2-(4-(1-Phenylethylamino)phenyl)ethanol (Table 4, Entry 2) ....................................................................................22

3-(1-Phenylethylamino)benzoic acid (Table 4, Entry 3) .......................................................................................23

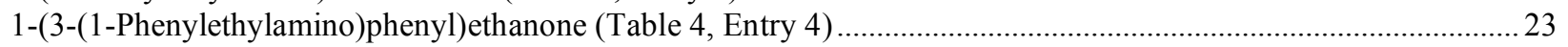

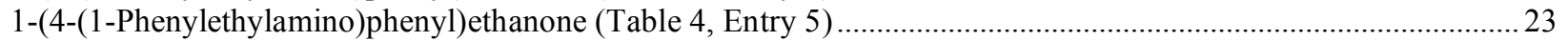

2-(Methylthio)-N-(1-phenylethyl)benzenamine (Table 4, Entry 6) ………………………………..............................2 24

4-(Methylthio)-N-(1-phenylethyl)benzenamine (Table 4, Entry 7) ……..................................................................... 24

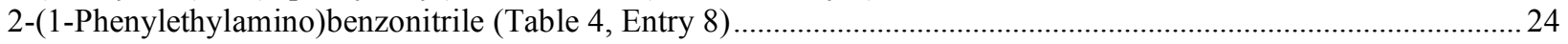

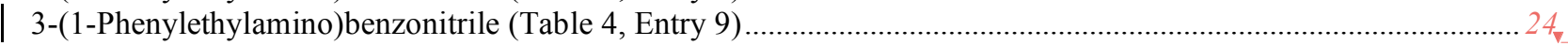

4-(1-Phenylethylamino)benzonitrile (Table 4, Entry 10) .......................................................................................25

Ethyl 2-(1-phenylethylamino)benzoate (Table 4, Entry 11) ....................................................................................2

Ethyl 3-(1-phenylethylamino)benzoate (Table 4, Entry 12) ........................................................................................25

| Ethyl 4-(1-phenylethylamino)benzoate (Table 4, Entry 13) ......................................................................................25

$N$-(3-(1-phenylethylamino)phenyl)acetamide (Table 4, Entry 14) ............................................................................. 26

4-(1-phenylethylamino)benzamide $\cdot 0.25 \mathrm{H}_{2} \mathrm{O}$ (Table 4, Entry 15) ........................................................................... 26

Determination of the Rate constants $\left(k_{\mathrm{obs}}\right)$ for the Nucleophilic Attack of Aniline and Substituted Anilines on

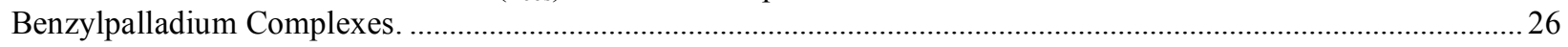

Determination of the Rate constants $\left(k_{\mathrm{obs}}\right)$ for the Nucleophilic Attack of Aniline and Substituted Anilines on

Allylpalladium Complexes. ................................................................................................................................2 27

Determination of the Rate constants $\left(k_{\mathrm{obs}}\right)$ for the Nucleophilic Attack of Aniline on 1,1-Dimethylallylpalladium

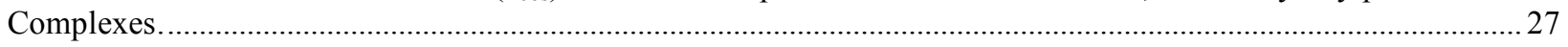

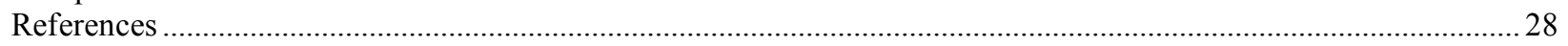

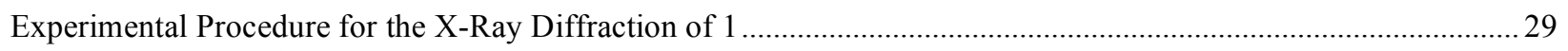

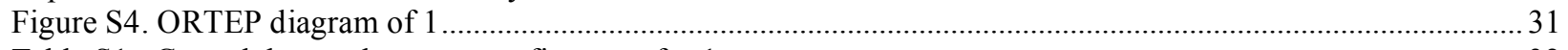

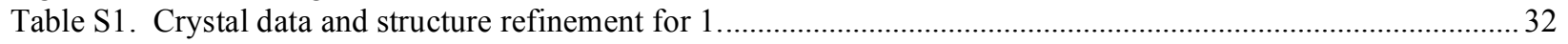

Table S2. Atomic coordinates ( $\left.\times 10^{4}\right)$ and equivalent isotropic displacement parameters $\left(\AA^{2} \times 10^{3}\right)$ for 1 . U(eq) is

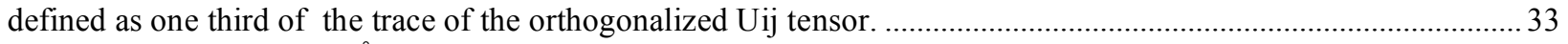

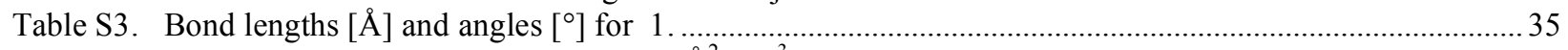

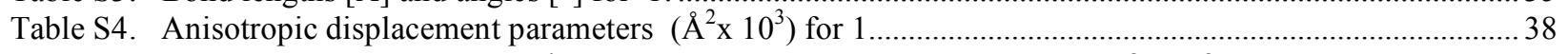

Table S5. Hydrogen coordinates $\left(\times 10^{4}\right)$ and isotropic displacement parameters $\left(\AA^{2} \times 10^{3}\right)$ for $1 \ldots \ldots \ldots \ldots \ldots \ldots \ldots \ldots . . . . . . . . . . .40$

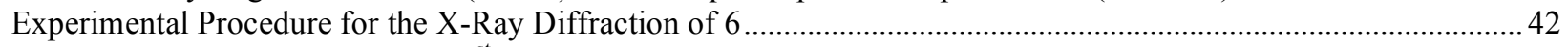

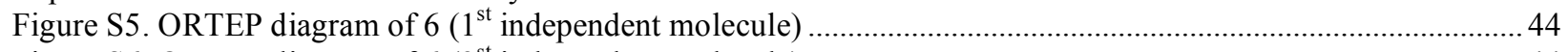

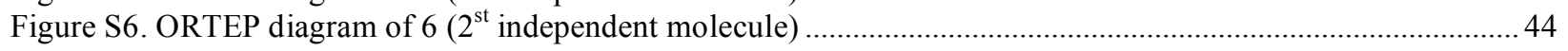

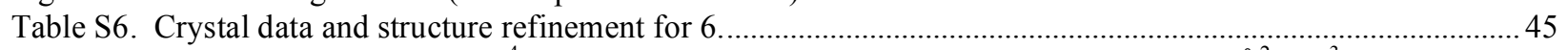

Table S7. Atomic coordinates $\left(\times 10^{4}\right)$ and equivalent isotropic displacement parameters $\left(\AA^{2} \times 10^{3}\right)$ for $6 \ldots \ldots \ldots \ldots . . . . .46$

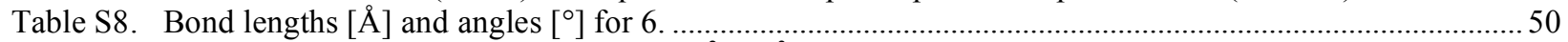

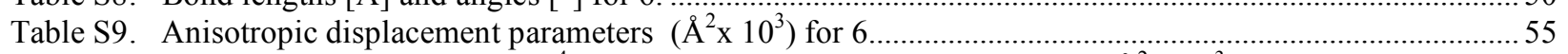

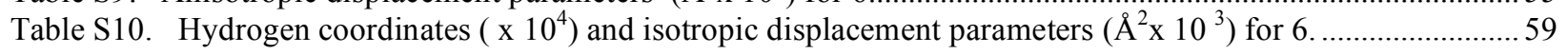

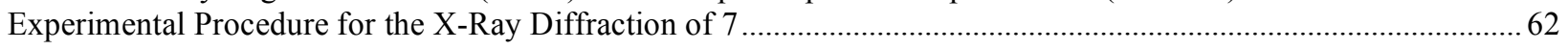

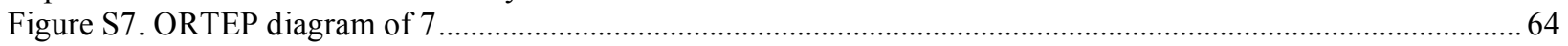

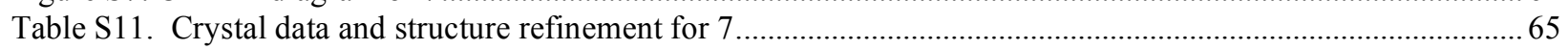

Table S12. Atomic coordinates ( $\times 10^{4}$ ) and equivalent isotropic displacement parameters $\left(\AA^{2} \times 10^{3}\right)$ for 7 . U(eq) is

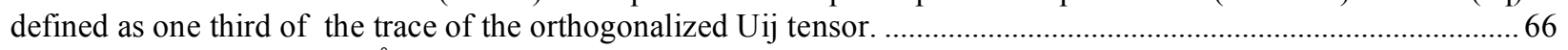

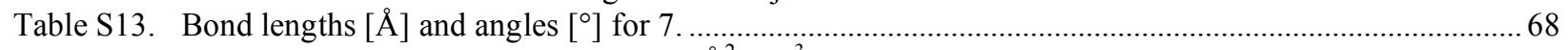

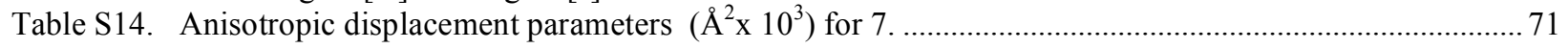

Table S15. Hydrogen coordinates $\left(\times 10^{4}\right)$ and isotropic displacement parameters $\left(\AA^{2} \times 10^{3}\right) \ldots \ldots \ldots \ldots \ldots \ldots \ldots \ldots \ldots \ldots . . .73$

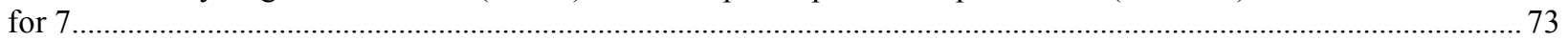

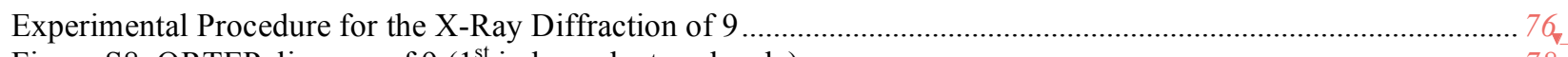

Figure S8. ORTEP diagram of 9 ( $1^{\text {st }}$ independent molecule $)$ 
Figure S9. ORTEP diagram of 9 ( $2^{\text {st }}$ independent molecule $)$

Table S16. Crystal data and structure refinement for 9 .

Table S17. Atomic coordinates $\left(\times 10^{4}\right)$ and equivalent isotropic displacement parameters $\left(\AA^{2} \times 10^{3}\right)$ for 9

Table S18. Bond lengths $[\AA]$ and angles $\left[{ }^{\circ}\right]$ for 9 .

Table S19. Anisotropic displacement parameters $\left(\AA^{2} \times 10^{3}\right)$ for 9

Table S20. Hydrogen coordinates $\left(\times 10^{4}\right)$ and isotropic displacement parameters $\left(\AA^{2} \times 10^{3}\right)$ for 9

Experimental Procedure for the X-Ray Diffraction of 12 .

Figure S10. ORTEP diagram of 12

Table S21. Crystal data and structure refinement for 12 .

Table S22. Atomic coordinates ( $\times 10^{4}$ ) and equivalent isotropic displacement parameters $\left(\AA^{2} \times 10^{3}\right)$ for 12 . U(eq)

is defined as one third of the trace of the orthogonalized Uij tensor.

Table S23. Bond lengths $[\AA]$ and angles $\left[{ }^{\circ}\right]$ for 12 .

Table S24. Anisotropic displacement parameters $\left(\AA^{2} \times 10^{3}\right)$ for 12 .

Table S25. Hydrogen coordinates $\left(\times 10^{4}\right)$ and isotropic displacement parameters $\left(\AA^{2} \times 10^{3}\right)$ for $12 \ldots \ldots \ldots \ldots \ldots \ldots . . . . .109$.

Experimental Procedure for the X-Ray Diffraction of 18 ...................................................................... 112

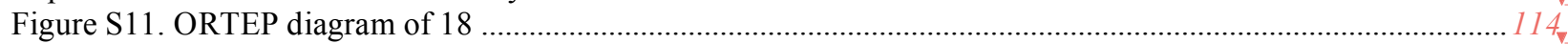

Table S26. Crystal data and structure refinement for 18 .

Table S27. Atomic coordinates ( x $10^{4}$ ) and equivalent isotropic displacement parameters $\left(\AA^{2} \times 10^{3}\right)$ for 18 . U(eq)

is defined as one third of the trace of the orthogonalized Uij tensor.

Table S28. Bond lengths $[\AA]$ and angles $\left[^{\circ}\right]$ for 18 .

Table S29. Anisotropic displacement parameters $\left(\AA^{2} \times 10^{3}\right)$ for 18 .

Table S30. Hydrogen coordinates $\left(\times 10^{4}\right)$ and isotropic displacement parameters $\left(\AA^{2} \times 10^{3}\right)$ for 18 .

Experimental Procedure for the X-Ray Diffraction of 24.

Figure S12. ORTEP diagram of 24

Table S31. Crystal data and structure refinement for 24.

Table S32. Atomic coordinates ( $\times 10^{4}$ ) and equivalent isotropic displacement parameters $\left(\AA^{2} \times 10^{3}\right)$ for 24 . U(eq) is defined as one third of the trace of the orthogonalized Uij tensor.

Table S33. Bond lengths $[\AA]$ and angles $\left[^{\circ}\right]$ for 24 .

Table S34. Anisotropic displacement parameters $\left(\AA^{2} \times 10^{3}\right)$ for 24.

Table S35. Hydrogen coordinates $\left(\times 10^{4}\right)$ and isotropic displacement parameters $\left(\AA^{2} \times 10^{3}\right)$ for 24

General Experimental Procedure and Reagent Availability. All manipulations were carried out under an inert atmosphere using a nitrogen-filled glovebox or standard Schlenk techniques. All glassware was oven or flame dried immediately prior to use. THF and diethyl ether were obtained as HPLC grade without inhibitors; benzene, toluene, dichloromethane, and pentane were obtained as ACS reagent grade. THF, diethyl ether, benzene, toluene, dichloromethane, and pentane were degassed by purging with nitrogen for $45 \mathrm{~min}$ and dried with a solvent purification system containing a $1 \mathrm{~m}$ column containing activated alumina. All reagents were obtained from commercial sources and used without further purification. ${ }^{1} \mathrm{H}$ NMR spectra were obtained at 400 or $500-\mathrm{MHz}$ and recorded relative to residual protio-solvent. ${ }^{13} \mathrm{C}$ NMR spectra were obtained at 101 or $126 \mathrm{MHz}$, and chemical shifts were recorded relative to the solvent resonance. ${ }^{31} \mathrm{P}$ NMR spectra were obtained at 122,162 or $202 \mathrm{MHz}$ and chemical shifts are reported relative to $85 \% \mathrm{H}_{3} \mathrm{PO}_{4} \cdot\left[\mathrm{Pd}\left(\eta^{3} \text {-allyl }\right) \mathrm{Cl}\right]_{2}{ }^{1},(\mathrm{COD}) \mathrm{PdCl}_{2}{ }^{2}(\mathrm{COD}=1,5-$ cyclooctadiene), (COD)Pd( $\eta^{3}$-allyl)OTf ${ }^{3},($ TMEDA $) \mathrm{PdBnBr}^{4},\left[\mathrm{Pd}\left(\eta^{3} \text {-cyclohexenyl }\right) \mathrm{Cl}\right]_{2}{ }^{5}$ and 
$\left[\mathrm{Pd}\left(\eta^{3}-1,1-\text { dimethylallyl }\right) \mathrm{Cl}\right]_{2}{ }^{6}$ were prepared using the reported procedures.

$(\mathrm{COD}) \mathrm{Pd}\left(\mathrm{CH}_{2} \mathrm{Ph}\right) \mathrm{Cl}^{7}$ and $\left[(\mathrm{DPPE}) \mathrm{Pd}\left(\eta^{3}\right.\right.$-allyl $\left.)\right] \mathrm{Cl}^{8}$ were prepared via alternate procedures to those found in the literature.

Preparation of [(Xantphos)Pd( $\eta^{3}$-allyl)Cl] (1). Into a $50 \mathrm{~mL}$ round bottom flask equipped with a magnetic stirbar was placed $\left[\operatorname{Pd}\left(\eta^{3} \text {-allyl }\right) \mathrm{Cl}\right]_{2}(200 . \mathrm{mg}, 0.547 \mathrm{mmol})$. Into a second $50 \mathrm{~mL}$ round bottom flask equipped with a magnetic stirbar was placed Xantphos (9,9-dimethyl-4,6bis(diphenylphosphino)xanthene) $(633 \mathrm{mg}, 1.09 \mathrm{mmol})$. Benzene $(15 \mathrm{~mL})$ was added to each flask, and the resulting solutions stirred for $10 \mathrm{~min}$ at $50{ }^{\circ} \mathrm{C}$ to ensure complete dissolution. The solutions were subsequently combined and an orange precipitate immediately began to form. After stirring for $30 \mathrm{~min}$ at $50{ }^{\circ} \mathrm{C}$, the resulting suspension was allowed to cool to room temperature, and the precipitate was isolated by filtration in air. The precipitate was washed two times with $30 \mathrm{~mL}$ of $\mathrm{Et}_{2} \mathrm{O}$ and dried in vacuo to give $807 \mathrm{mg}$ (97\%) of $\mathbf{1}$. Single crystals suitable for X-ray diffraction were obtained by allowing $\mathrm{Et}_{2} \mathrm{O}$ to diffuse into a saturated solution of $\mathbf{1}$ in benzene. ${ }^{1} \mathrm{H}$ NMR (400 MHz, CDCl 3 , TMS) $\delta 1.65$ (s, 6H), 3.60 (br s, $4 \mathrm{H}$ ), 5.70 (qt, $J=10.0 \mathrm{~Hz}$, $1 \mathrm{H}), 6.53(\mathrm{t}, J=9.0 \mathrm{~Hz}, 2 \mathrm{H}), 7.08(\mathrm{t}, J=7.8 \mathrm{~Hz}, 2 \mathrm{H}), 7.31(\mathrm{~m}, 20 \mathrm{H}), 7.55(\mathrm{dd}, J=7.6,1.2 \mathrm{~Hz}$, $2 \mathrm{H}) ;{ }^{13} \mathrm{C}\left\{{ }^{1} \mathrm{H}\right\} \operatorname{NMR}\left(101 \mathrm{MHz}, \mathrm{CD}_{2} \mathrm{Cl}_{2}\right) \delta 22.7,36.5,77.2(\mathrm{t}, J=13.2 \mathrm{~Hz}), 115.5,120.4(\mathrm{~m})$, 124.6 (t, $J=2.8 \mathrm{~Hz}), 127.8,128.8$ (t, $J=4.0 \mathrm{~Hz}), 130.4,132.1,133.0(\mathrm{~m}), 133.5$ (t, $J=5.0 \mathrm{~Hz}$ ), $134.6(\mathrm{t}, J=2.0 \mathrm{~Hz}), 155.7(\mathrm{t}, J=3.5 \mathrm{~Hz}) ;{ }^{31} \mathrm{P}\left\{{ }^{1} \mathrm{H}\right\} \mathrm{NMR}\left(122 \mathrm{MHz}, \mathrm{CDCl}_{3}\right) \delta$ 4.584; Anal. Calcd for $\mathrm{C}_{42} \mathrm{H}_{37} \mathrm{ClOP}_{2} \mathrm{Pd}$ : C, 66.24; H, 4.90 Found: C, 65.89; H, 4.75.

Preparation of [(Xantphos)Pd( $\eta^{3}$-allyl)]OTf (2). Into a $20 \mathrm{~mL}$ scintillation vial equipped with a magnetic stirbar was placed 1 (100. $\mathrm{mg}, 0.131 \mathrm{mmol})$ and $\mathrm{AgOTF}(33.7 \mathrm{mg}, 0.131 \mathrm{mmol})$. Dichloromethane $(8 \mathrm{~mL})$ was added, and the orange suspension was stirred for $20 \mathrm{~min}$. Filtration through Celite yielded a clear orange solution, which was concentrated to $1 \mathrm{~mL}$ under reduced pressure, layered with $\mathrm{Et}_{2} \mathrm{O}$, and cooled at $-35^{\circ} \mathrm{C}$ for $12 \mathrm{~h}$. The resulting orange crystals were separated from the supernatant, washed with $2 \times 10 \mathrm{~mL} \mathrm{Et}_{2} \mathrm{O}$, and dried to give $99.2 \mathrm{mg}$ (86\%) of 2. ${ }^{1} \mathrm{H}$ NMR (500 MHz, $\left.\mathrm{CD}_{2} \mathrm{Cl}_{2}\right) \delta 1.54$ (s, 3H), $1.82(\mathrm{~s}, 3 \mathrm{H}), 3.48(\mathrm{~m}, 2 \mathrm{H}), 3.78$ (dd, $J=$ 6.6, $3.6 \mathrm{~Hz}, 2 \mathrm{H}), 5.90$ (sept, $J=7.0 \mathrm{~Hz}, 1 \mathrm{H}), 6.61(\mathrm{~m}, 2 \mathrm{H}), 7.09$ (m, 4H), 7.20 (t, $J=7.5 \mathrm{~Hz}$, 2H), $7.23(\mathrm{~m}, 4 \mathrm{H}), 7.28(\mathrm{t}, J=6.8 \mathrm{~Hz}, 4 \mathrm{H}), 7.39(\mathrm{t}, J=7.5 \mathrm{~Hz}, 2 \mathrm{H}), 7.44(\mathrm{t}, J=7.5 \mathrm{~Hz}, 4 \mathrm{H}), 7.50$ (t, $J=7.0 \mathrm{~Hz}, 2 \mathrm{H}), 7.70(\mathrm{dd}, J=7.5,1.5 \mathrm{~Hz}, 2 \mathrm{H}) ;{ }^{13} \mathrm{C}\left\{{ }^{1} \mathrm{H}\right\}$ NMR $\left(126 \mathrm{MHz}, \mathrm{CD}_{2} \mathrm{Cl}_{2}\right) \delta 25.7$, 30.1, 36.4, 79.8 (t, $J=14.6 \mathrm{~Hz}), 118.0(\mathrm{~m}), 121.4$ (q, $J=320.5 \mathrm{~Hz}), 122.6$ (t, $J=4.3 \mathrm{~Hz}), 125.3$ (t, $J=3.8 \mathrm{~Hz}), 128.9,129.4$ (t, $J=5.2 \mathrm{~Hz}), 129.8(\mathrm{~m}), 131.2(\mathrm{~d}, J=8.9 \mathrm{~Hz}), 132.4,132.7(\mathrm{~m})$, 
$132.8(\mathrm{t}, J=6.5 \mathrm{~Hz}), 133.3(\mathrm{t}, J=7.0 \mathrm{~Hz}), 134.7(\mathrm{t}, J=1.9 \mathrm{~Hz}), 155.5(\mathrm{t}, J=3.6 \mathrm{~Hz}) ;{ }^{31} \mathrm{P}\left\{{ }^{1} \mathrm{H}\right\}$ NMR (202 MHz, $\mathrm{CD}_{2} \mathrm{Cl}_{2}$ ) $\delta$ 4.25; Anal. Calcd for $\mathrm{C}_{43} \mathrm{H}_{37} \mathrm{~F}_{3} \mathrm{O}_{4} \mathrm{P}_{2} \mathrm{PdS}: \mathrm{C}$, 59.01; H, 4.26 Found: C, 58.72; H, 4.26.

Preparation of [(DPPF)Pd( $\eta^{3}$-phenethyl)]OTf (3). Into a $50 \mathrm{~mL}$ round bottom flask equipped with a magnetic stirbar was placed $\mathrm{CpPd}\left(\eta^{3}\right.$-allyl $)(50.0 \mathrm{mg}, 0.235 \mathrm{mmol})$ and styrene $(1.30 \mathrm{~mL}$, $11.3 \mathrm{mmol}$ ). Into a second $50 \mathrm{~mL}$ round bottom flask equipped with a magnetic stirbar was placed DPPF (130. mg, $0.235 \mathrm{mmol})$. Tetrahydrofuran $(15 \mathrm{~mL})$ was added to each flask, and the resulting solutions stirred for $20 \mathrm{~min}$ to ensure complete dissolution. The solutions were subsequently combined and stirred at $50{ }^{\circ} \mathrm{C}$ for $1 \mathrm{~h}$. After cooling the solution to $22{ }^{\circ} \mathrm{C}$, a tetrahydrofuran solution $(0.5 \mathrm{~mL})$ of anilinium triflate $(57.2 \mathrm{mg}, 0.235 \mathrm{mmol})$ was slowly added via syringe. After $6 \mathrm{~h}$ of stirring at $22^{\circ} \mathrm{C}$, all volatile materials were removed, and the residue was dissolved in dichloromethane $(10 \mathrm{~mL})$. Filtration through Celite yielded a clear solution, which was concentrated to $2 \mathrm{~mL}$ under reduced pressure, layered with $\mathrm{Et}_{2} \mathrm{O}$, and cooled at $-35^{\circ} \mathrm{C}$ for $12 \mathrm{~h}$. The resulting orange crystals were isolated from the supernatant, washed with $2 \times 10$ $\mathrm{mL} \mathrm{Et}{ }_{2} \mathrm{O}$, and dried to give $166 \mathrm{mg}$ (77\%) of 3. ${ }^{1} \mathrm{H} \mathrm{NMR}\left(500 \mathrm{MHz}, \mathrm{CD}_{2} \mathrm{Cl}_{2}\right) \delta 0.86$ (ddd, $J=$ 12.3, 11.0, $6.7 \mathrm{~Hz}, 3 \mathrm{H}), 3.72(\mathrm{~m}, 2 \mathrm{H}), 3.90(\mathrm{~m}, 1 \mathrm{H}), 4.30(\mathrm{~m}, 2 \mathrm{H}), 4.45(\mathrm{~m}, 1 \mathrm{H}), 4.55(\mathrm{~m}, 1 \mathrm{H})$, $4.57(\mathrm{~m}, 1 \mathrm{H}), 4.66(\mathrm{~m}, 1 \mathrm{H}), 6.75(\mathrm{t}, J=6.0 \mathrm{~Hz}, 1 \mathrm{H}), 6.91(\mathrm{~m}, 2 \mathrm{H}), 6.96(\mathrm{~m}, 2 \mathrm{H}), 7.00(\mathrm{~m}, 1 \mathrm{H})$, 7.35-7.63 (m, 19H); ${ }^{13} \mathrm{C}\left\{{ }^{1} \mathrm{H}\right\}$ NMR $\left(126 \mathrm{MHz}, \mathrm{CD}_{2} \mathrm{Cl}_{2}\right) \delta 14.4(\mathrm{~d}, J=6.4 \mathrm{~Hz}), 70.7(\mathrm{dd}, J=$ 40.7, 8.6 Hz), 72.9 (d, $J=5.8 \mathrm{~Hz}), 73.7$ (d, $J=6.6 \mathrm{~Hz}), 74.1$ (d, $J=7.1 \mathrm{~Hz}), 74.3$ (dd, $J=49.8$, $2.8 \mathrm{~Hz}), 74.8(\mathrm{~d}, J=8.7 \mathrm{~Hz}), 74.8(\mathrm{~d}, J=11.2 \mathrm{~Hz}), 75.0(\mathrm{~d}, J=9.3 \mathrm{~Hz}), 75.5(\mathrm{dd}, J=55.1,4.4$ Hz), $76.3(\mathrm{~d}, J=9.2 \mathrm{~Hz}), 77.1(\mathrm{~d}, J=15.5 \mathrm{~Hz}), 109.8(\mathrm{~d}, J=10.1 \mathrm{~Hz}), 121.2(\mathrm{t}, J=6.4 \mathrm{~Hz})$, 121.5 (q, $J=321.5 \mathrm{~Hz}$ ), 122.3 (d, $J=4.5 \mathrm{~Hz}$ ), 129-135 (aromatic carbon resonances; because all aryl resonances are inequivalent and coupled to phosphorus these resonances were unresolved); ${ }^{31} \mathrm{P}\left\{{ }^{1} \mathrm{H}\right\}$ NMR $\left(202 \mathrm{MHz}, \mathrm{CD}_{2} \mathrm{Cl}_{2}\right) \delta 22.9(\mathrm{~d}, J=53.9 \mathrm{~Hz}), 33.6(\mathrm{~d}, J=54.7 \mathrm{~Hz})$; Anal. Calcd for $\mathrm{C}_{43} \mathrm{H}_{37} \mathrm{~F}_{3} \mathrm{FeO}_{3} \mathrm{P}_{2} \mathrm{PdS}: \mathrm{C}, 56.44 ; \mathrm{H}, 4.08$ Found: C, 56.61; H, 4.28.

Preparation of [(Xantphos)Pd( $\boldsymbol{\eta}^{3}$-benzyl)CI] (4). Into a $20 \mathrm{~mL}$ scintillation vial equipped with a magnetic stirbar was placed $(\mathrm{COD}) \mathrm{Pd}\left(\mathrm{CH}_{2} \mathrm{Ph}\right) \mathrm{Cl}(50.0 \mathrm{mg}, 0.147 \mathrm{mmol})$ and Xantphos $(84.8$ $\mathrm{mg}, 0.147 \mathrm{mmol})$. Dichloromethane $(4 \mathrm{~mL})$ was added, and the solution was stirred for $10 \mathrm{~min}$. Filtration through Celite yielded a clear solution, which was concentrated to $1 \mathrm{~mL}$ under reduced pressure, layered with pentane, and cooled at $-35^{\circ} \mathrm{C}$ for $8 \mathrm{~h}$. The resulting brown crystals were isolated from the supernatant, washed with 2 x $5 \mathrm{~mL}$ pentane, and dried to give $97.2 \mathrm{mg}(82 \%)$ 
of 4. ${ }^{1} \mathrm{H}$ NMR (500 MHz, $\left.\mathrm{CD}_{2} \mathrm{Cl}_{2}\right) \delta 1.71(\mathrm{~s}, 6 \mathrm{H}), 3.00(\mathrm{~s}, 2 \mathrm{H}), 6.51(\mathrm{~d}, J=7.0 \mathrm{~Hz}, 2 \mathrm{H}), 6.68(\mathrm{t}$, $J=7.5 \mathrm{~Hz}, 2 \mathrm{H}), 6.82(\mathrm{~m}, 1 \mathrm{H}), 6.93(\mathrm{~m}, 2 \mathrm{H}), 7.12(\mathrm{t}, J=7.8 \mathrm{~Hz}, 2 \mathrm{H}), 7.27(\mathrm{t}, J=7.3 \mathrm{~Hz}, 8 \mathrm{H})$, $7.34(\mathrm{t}, J=7.3 \mathrm{~Hz}, 4 \mathrm{H}), 7.47(\mathrm{~m}, 8 \mathrm{H}), 7.57(\mathrm{dd}, J=7.8,1.3 \mathrm{~Hz}, 2 \mathrm{H}) ;{ }^{13} \mathrm{C}\left\{{ }^{1} \mathrm{H}\right\} \mathrm{NMR}(126 \mathrm{MHz}$, THF-d $\left.\mathrm{d}_{8}\right) \delta 28.7,36.9,38.1(\mathrm{t}, J=14.2 \mathrm{~Hz}), 123.6(\mathrm{t}, J=18.8 \mathrm{~Hz}), 124.7,126.1,127.6,128.5$, $129.1(\mathrm{t}, J=5.3 \mathrm{~Hz}), 130.4,130.5,132.2,133.4(\mathrm{t}, J=18.3 \mathrm{~Hz}), 133.3,135.5(\mathrm{t}, J=6.9 \mathrm{~Hz})$, 138.9, $156.2(\mathrm{t}, J=5.7 \mathrm{~Hz}) ;{ }^{31} \mathrm{P}\left\{{ }^{1} \mathrm{H}\right\}$ NMR $\left(202 \mathrm{MHz}, \mathrm{CD}_{2} \mathrm{Cl}_{2}\right) \delta$ 12.9; Anal. Calcd for $\mathrm{C}_{46} \mathrm{H}_{39} \mathrm{ClOP}_{2} \mathrm{Pd}: \mathrm{C}, 68.07 ; \mathrm{H}, 4.84$ Found: C, 67.82; H, 4.72.

Preparation of $\left[(\right.$ Xantphos $) P d\left(\eta^{3}\right.$-benzyl) $] B^{-} F_{4}$ (5). Into a $20 \mathrm{~mL}$ scintillation vial equipped with a magnetic stirbar was placed $(\mathrm{COD}) \mathrm{Pd}\left(\mathrm{CH}_{2} \mathrm{Ph}\right) \mathrm{Cl}(100 . \mathrm{mg}, 0.293 \mathrm{mmol})$ and Xantphos (170. $\mathrm{mg}, 0.293 \mathrm{mmol})$. Dichloromethane $(5 \mathrm{~mL})$ was added, and the solution was stirred for 5 min. $\mathrm{AgBF}_{4}(57.1 \mathrm{mg}, 0.293 \mathrm{mmol})$ was added to the stirring solution, and the resulting suspension was stirred for $10 \mathrm{~min}$. Filtration through Celite yielded a clear solution, which was concentrated to $2 \mathrm{~mL}$ under reduced pressure, layered with $\mathrm{Et}_{2} \mathrm{O}$, and cooled at $-35^{\circ} \mathrm{C}$ for $6 \mathrm{~h}$. Black crystals of an unidentified impurity precipitated and were removed by filtration. The mother liquor was again layered with $\mathrm{Et}_{2} \mathrm{O}$ and cooled at $-35^{\circ} \mathrm{C}$ for $12 \mathrm{~h}$. The resulting white crystals were isolated from the supernatant, washed with $2 \times 10 \mathrm{~mL} \mathrm{Et}_{2} \mathrm{O}$, and dried to give 162 mg (64\%) of 5. ${ }^{1} \mathrm{H}$ NMR (500 MHz, $\left.\mathrm{CD}_{2} \mathrm{Cl}_{2}\right) \delta 1.65(\mathrm{~s}, 6 \mathrm{H}), 3.05(\mathrm{t}, J=3.3 \mathrm{~Hz}, 2 \mathrm{H}), 6.69(\mathrm{~m}$, 2H), $6.79(\mathrm{~m}, 2 \mathrm{H}), 6.85(\mathrm{t}, J=7.8 \mathrm{~Hz}, 2 \mathrm{H}), 7.01(\mathrm{~m}, 1 \mathrm{H}), 7.06(\mathrm{~m}, 8 \mathrm{H}), 7.20(\mathrm{dt}, J=7.8,1.5 \mathrm{~Hz}$, 2H), $7.34(\mathrm{dt}, J=7.8,1.5 \mathrm{~Hz}, 8 \mathrm{H}), 7.45(\mathrm{~m}, 4 \mathrm{H}), 7.67(\mathrm{dd}, J=8.0,1.0 \mathrm{~Hz}, 2 \mathrm{H}) ;{ }^{13} \mathrm{C}\left\{{ }^{1} \mathrm{H}\right\} \mathrm{NMR}$ $\left(126 \mathrm{MHz}, \mathrm{CD}_{2} \mathrm{Cl}_{2}\right) \delta 28.4,36.3,54.8$ (t, $\left.J=17.9 \mathrm{~Hz}\right), 118.4(\mathrm{~d}, J=40.3 \mathrm{~Hz}), 121.6,123.1$, $125.4(\mathrm{~d}, J=6.8 \mathrm{~Hz}), 129.1,129.3(\mathrm{~d}, J=10.6 \mathrm{~Hz}), 129.8$ (d, $J=42.5 \mathrm{~Hz}), 131.3,132.6,132.7$ (t, $J=3.4 \mathrm{~Hz}), 133.2(\mathrm{t}, J=4.3 \mathrm{~Hz}), 133.4(\mathrm{~d}, J=12.6 \mathrm{~Hz}), 134.5(\mathrm{~d}, J=3.8 \mathrm{~Hz}), 155.2(\mathrm{~d}, J=$ $8.8 \mathrm{~Hz}) ;{ }^{31} \mathrm{P}\left\{{ }^{1} \mathrm{H}\right\}$ NMR $\left(202 \mathrm{MHz}, \mathrm{CD}_{2} \mathrm{Cl}_{2}\right) \delta 11.1$; Anal. Calcd for $\mathrm{C}_{46} \mathrm{H}_{39} \mathrm{BF}_{4} \mathrm{OP}_{2} \mathrm{PdS}: \mathrm{C}$, 64.02; H, 4.56 Found: C, 63.74; H, 4.26.

Preparation of [(Xantphos)Pd( $\boldsymbol{\eta}^{3}$-benzyl)]OTf (6). Into a $20 \mathrm{~mL}$ scintillation vial equipped with a magnetic stirbar was placed (COD) $\mathrm{Pd}\left(\mathrm{CH}_{2} \mathrm{Ph}\right) \mathrm{Cl}$ (300. mg, $\left.0.879 \mathrm{mmol}\right)$, Xantphos (509 $\mathrm{mg}, 0.879 \mathrm{mmol})$ and AgOTF (226 mg , $0.879 \mathrm{mmol})$. Dichloromethane (12 mL) was added, and the orange suspension was stirred for $20 \mathrm{~min}$. Filtration through Celite yielded a clear orange solution, which was concentrated to $1 \mathrm{~mL}$ under reduced pressure, layered with $\mathrm{Et}_{2} \mathrm{O}$, and cooled at $-35{ }^{\circ} \mathrm{C}$ for $12 \mathrm{~h}$. The resulting white crystals were isolated from the supernatant, washed with $2 \times 10 \mathrm{~mL} \mathrm{Et}_{2} \mathrm{O}$, and dried to give $774 \mathrm{mg}$ (95\%) of 6 . Single crystals suitable for 
X-ray diffraction were obtained by allowing $\mathrm{Et}_{2} \mathrm{O}$ to diffuse into a saturated solution of $\mathbf{6}$ in dichloromethane. ${ }^{1} \mathrm{H}$ NMR (500 MHz, $\left.\mathrm{CD}_{2} \mathrm{Cl}_{2}\right) \delta 1.65(\mathrm{~s}, 6 \mathrm{H}), 3.06(\mathrm{t}, J=3.3 \mathrm{~Hz}, 2 \mathrm{H}), 6.70(\mathrm{~m}$, 2H), $6.79(\mathrm{~m}, 2 \mathrm{H}), 6.84(\mathrm{t}, J=7.8 \mathrm{~Hz}, 2 \mathrm{H}), 7.01(\mathrm{~m}, 1 \mathrm{H}), 7.07(\mathrm{~m}, 8 \mathrm{H}), 7.20(\mathrm{dt}, J=7.8,1.5 \mathrm{~Hz}$, 2H), $7.34(\mathrm{dt}, J=7.8,2.0 \mathrm{~Hz}, 8 \mathrm{H}), 7.45(\mathrm{~m}, 4 \mathrm{H}), 7.67(\mathrm{dd}, J=8.0,1.0 \mathrm{~Hz}, 2 \mathrm{H}) ;{ }^{13} \mathrm{C}\left\{{ }^{1} \mathrm{H}\right\} \mathrm{NMR}$ $\left(126 \mathrm{MHz}, \mathrm{CD}_{2} \mathrm{Cl}_{2}\right) \delta 28.4,36.3,54.7(\mathrm{t}, J=17.1 \mathrm{~Hz}), 118.4(\mathrm{~d}, J=41.2 \mathrm{~Hz}), 121.5(\mathrm{q}, J=$ $321.6 \mathrm{~Hz}), 121.5,122.9,125.4$ (d, $J=7.3 \mathrm{~Hz}), 129.0,129.3$ (d, $J=10.4 \mathrm{~Hz}), 129.9$ (d, $J=42.9$ $\mathrm{Hz}), 131.3(\mathrm{~d}, J=2.6 \mathrm{~Hz}), 132.5,132.7(\mathrm{t}, J=2.9 \mathrm{~Hz}), 133.2(\mathrm{t}, J=3.8 \mathrm{~Hz}), 133.4$ (d, $J=13.2$ $\mathrm{Hz}), 134.5(\mathrm{~d}, J=4.1 \mathrm{~Hz}), 155.2(\mathrm{~d}, J=8.3 \mathrm{~Hz}) ;{ }^{31} \mathrm{P}\left\{{ }^{1} \mathrm{H}\right\}$ NMR $\left(202 \mathrm{MHz}, \mathrm{CD}_{2} \mathrm{Cl}_{2}\right) \delta 13.3$; Anal. Calcd for $\mathrm{C}_{47} \mathrm{H}_{39} \mathrm{~F}_{3} \mathrm{O}_{4} \mathrm{P}_{2} \mathrm{PdS}$ : C, 61.01; H, 4.25 Found: C, 60.77; H, 4.04.

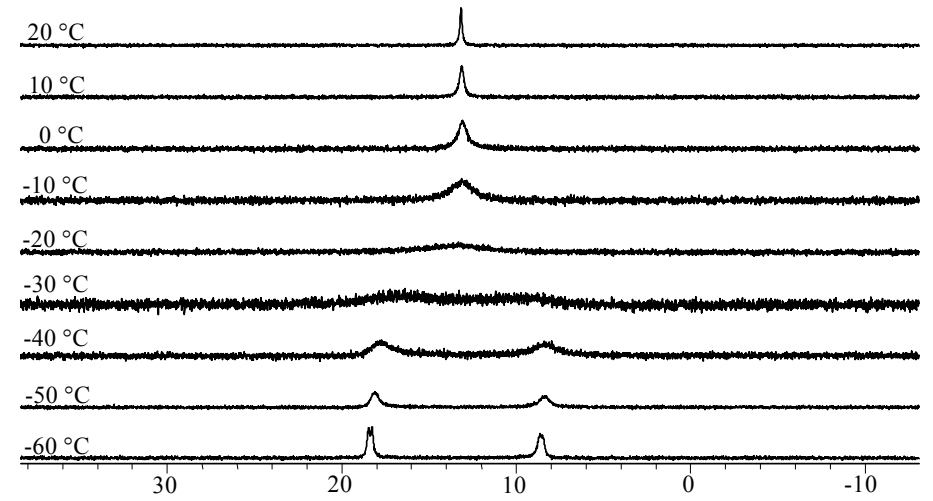

Figure S1: $162 \mathrm{MHz}{ }^{31} \mathrm{P}$ NMR spectra of XantphosPd( $\eta^{3}$-benzyl)OTf (6) at various temperatures in $\mathrm{CD}_{2} \mathrm{Cl}_{2}$.

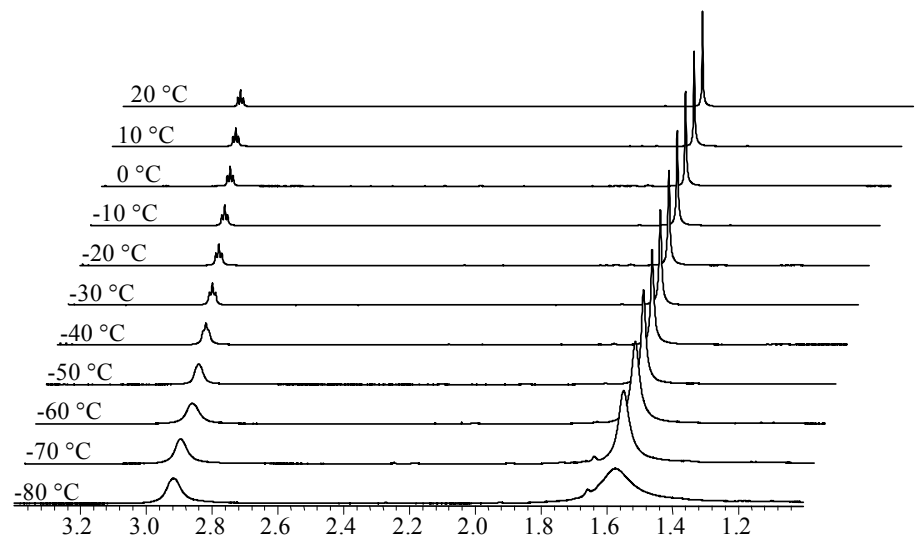

Figure S2: $500 \mathrm{MHz}^{1} \mathrm{H}$ NMR spectra of XantphosPd( $\left(\eta^{3}\right.$-benzyl)OTf (6) at various temperatures in $\mathrm{CD}_{2} \mathrm{Cl}_{2}$.

Preparation of [(DPEphos)Pd( $\eta^{3}$-benzyl)]OTf $\cdot \mathbf{C H}_{2} \mathbf{C l}_{\mathbf{2}}$ (7). Into a $20 \mathrm{~mL}$ scintillation vial equipped with a magnetic stirbar was placed $(\mathrm{COD}) \mathrm{Pd}\left(\mathrm{CH}_{2} \mathrm{Ph}\right) \mathrm{Cl}(50.0 \mathrm{mg}, 0.147 \mathrm{mmol})$ and 
DPEphos (78.9 mg, $0.147 \mathrm{mmol})$. Dichloromethane $(5 \mathrm{~mL})$ was added, and the solution was stirred for $10 \mathrm{~min}$. AgOTF (37.7 $\mathrm{mg}, 0.147 \mathrm{mmol}$ ) was added to the stirring solution and the resulting suspension was stirred for $10 \mathrm{~min}$. Filtration through Celite yielded a clear orange solution, which was concentrated to $1 \mathrm{~mL}$ under reduced pressure, layered with pentane, and cooled at $-35{ }^{\circ} \mathrm{C}$ for $12 \mathrm{~h}$. The resulting yellow crystals were isolated from the supernatant, washed with $2 \times 5 \mathrm{~mL}$ pentane, and dried to give $133 \mathrm{mg}$ (93\%) of 7. Single crystals suitable for $\mathrm{X}$-ray diffraction were obtained by allowing pentane to diffuse into a saturated solution of $\mathbf{7}$ in dichloromethane. ${ }^{1} \mathrm{H}$ NMR $\left(500 \mathrm{MHz}, \mathrm{C}_{2} \mathrm{D}_{2} \mathrm{Cl}_{4}, 75^{\circ} \mathrm{C}\right) \delta 3.05(\mathrm{t}, J=7.3 \mathrm{~Hz}, 2 \mathrm{H}), 6.77(\mathrm{~m}, 4 \mathrm{H})$, $6.84(\mathrm{dd}, J=7.8,3.8 \mathrm{~Hz}, 2 \mathrm{H}), 6.99(\mathrm{~m}, 5 \mathrm{H}), 7.16(\mathrm{dd}, J=10.3,8.3 \mathrm{~Hz}, 8 \mathrm{H}), 7.32(\mathrm{t}, J=7.8 \mathrm{~Hz}$, $2 \mathrm{H}), 7.46-7.55(\mathrm{~m}, 12 \mathrm{H}) ;{ }^{13} \mathrm{C}\left\{{ }^{1} \mathrm{H}\right\} \operatorname{NMR}\left(126 \mathrm{MHz}, \mathrm{C}_{2} \mathrm{D}_{2} \mathrm{Cl}_{4}, 75{ }^{\circ} \mathrm{C}\right) \delta 56.5(\mathrm{t}, J=22.4 \mathrm{~Hz})$, $118.6(\mathrm{t}, J=6.3 \mathrm{~Hz}), 120.2,120.3,120.8(\mathrm{q}, J=322.9 \mathrm{~Hz}), 121.8(\mathrm{~d}, J=43.5 \mathrm{~Hz}), 124.9$ (d, $J=$ $6.8 \mathrm{~Hz}), 128.9$ (d, $J=10.6 \mathrm{~Hz}), 129.2(\mathrm{~d}, J=44.7 \mathrm{~Hz}), 131.1,132.7$ (t, $J=3.5 \mathrm{~Hz}), 132.9,133.0$ (t, $J=13.4 \mathrm{~Hz}), 133.2(\mathrm{t}, J=4.3 \mathrm{~Hz}), 134.1,158.2(\mathrm{~d}, J=8.6 \mathrm{~Hz}) ;{ }^{31} \mathrm{P}\left\{{ }^{1} \mathrm{H}\right\} \mathrm{NMR}(202 \mathrm{MHz}$, $\left.\mathrm{C}_{2} \mathrm{D}_{2} \mathrm{Cl}_{4}, 75{ }^{\circ} \mathrm{C}\right) \delta 16.3$; Anal. Calcd for $\mathrm{C}_{45} \mathrm{H}_{37} \mathrm{Cl}_{2} \mathrm{~F}_{3} \mathrm{O}_{4} \mathrm{P}_{2} \mathrm{PdS}$ : C, 55.71; H, 3.84 Found: $\mathrm{C}$, $55.69 ; \mathrm{H}, 3.79$.

Preparation of [(DPPF)Pd(benzyl)Cl] (8). Into a $20 \mathrm{~mL}$ scintillation vial equipped with a magnetic stirbar was placed (COD) $\mathrm{Pd}\left(\mathrm{CH}_{2} \mathrm{Ph}\right) \mathrm{Cl}(50.0 \mathrm{mg}, 0.147 \mathrm{mmol})$ and DPPF $(81.2 \mathrm{mg}$, $0.147 \mathrm{mmol})$. Dichloromethane $(4 \mathrm{~mL})$ was added, and the solution was stirred for $10 \mathrm{~min}$. Filtration through Celite yielded a clear solution, which was concentrated to $1 \mathrm{~mL}$ under reduced pressure, layered with pentane, and cooled at $-35^{\circ} \mathrm{C}$ for $8 \mathrm{~h}$. The resulting orange crystals were isolated from the supernatant, washed with 2 x $5 \mathrm{~mL}$ pentane, and dried to give $105 \mathrm{mg}$ (91\%) of 8. ${ }^{1} \mathrm{H}$ NMR (500 MHz, $\mathrm{CD}_{2} \mathrm{Cl}_{2}$ ) $\delta 3.20$ (s, $\left.2 \mathrm{H}\right), 3.40$ (q, $\left.J=5.0 \mathrm{~Hz}, 2 \mathrm{H}\right), 4.08$ (s, 2H), 4.39 (s, 2H), $4.45(\mathrm{~s}, 2 \mathrm{H}), 7.01-7.11(\mathrm{~m}, 5 \mathrm{H}), 7.28(\mathrm{td}, J=7.8,1.0 \mathrm{~Hz}, 4 \mathrm{H}), 7.37-7.50(\mathrm{~m}, 8 \mathrm{H}), 7.60(\mathrm{dd}$, $J=11.3,7.8 \mathrm{~Hz}, 4 \mathrm{H}), 7.85(\mathrm{t}, J=8.8 \mathrm{~Hz}, 4 \mathrm{H}) ;{ }^{13} \mathrm{C}\left\{{ }^{1} \mathrm{H}\right\} \mathrm{NMR}\left(126 \mathrm{MHz}, \mathrm{THF}-\mathrm{d}_{8}\right) \delta 40.6(\mathrm{dd}, J$ $=88.6,4.2 \mathrm{~Hz}), 72.2(\mathrm{~d}, J=3.9 \mathrm{~Hz}), 73.8(\mathrm{~d}, J=7.4 \mathrm{~Hz}), 74.9(\mathrm{~d}, J=7.1 \mathrm{~Hz}), 75.5(\mathrm{dd}, J=53.4$, $5.6 \mathrm{~Hz}), 76.1(\mathrm{~d}, J=34.3 \mathrm{~Hz}), 77.0(\mathrm{~d}, J=13.0 \mathrm{~Hz}), 124.4(\mathrm{t}, J=2.9 \mathrm{~Hz}), 127.9,128.4(\mathrm{~d}, J=$ $9.3 \mathrm{~Hz}), 128.5(\mathrm{~d}, J=10.3 \mathrm{~Hz}), 129.6(\mathrm{dd}, J=4.7,2.3 \mathrm{~Hz}), 130.3(\mathrm{~d}, J=1.8 \mathrm{~Hz}), 131.1$ (d, $J=$ $2.6 \mathrm{~Hz}), 133.6$ (d, $J=31.5 \mathrm{~Hz}), 134.3(\mathrm{~d}, J=50.3 \mathrm{~Hz}), 135.0$ (d, $J=12.1 \mathrm{~Hz}), 135.5$ (d, $J=13.0$ $\mathrm{Hz}), 144.8(\mathrm{~d}, J=11.8 \mathrm{~Hz}) ;{ }^{31} \mathrm{P}\left\{{ }^{1} \mathrm{H}\right\} \mathrm{NMR}\left(202 \mathrm{MHz}, \mathrm{CD}_{2} \mathrm{Cl}_{2}\right) \delta 13.5(\mathrm{~d}, J=51.5 \mathrm{~Hz}), 34.7$ (d, $J=51.7 \mathrm{~Hz}$ ); Anal. Calcd for $\mathrm{C}_{41} \mathrm{H}_{35} \mathrm{ClFeP}_{2} \mathrm{Pd}: \mathrm{C}, 62.54 ; \mathrm{H}, 4.48$ Found: C, 62.67; H, 4.54. 
Preparation of [(DPPF)Pd $\left(\eta^{3}\right.$-benzyl)]OTf $\cdot \mathbf{0 . 5} \mathbf{C H}_{2} \mathbf{C l}_{\mathbf{2}}$ (9). Into a $20 \mathrm{~mL}$ scintillation vial equipped with a magnetic stirbar was placed (COD) $\mathrm{Pd}\left(\mathrm{CH}_{2} \mathrm{Ph}\right) \mathrm{Cl}(300 . \mathrm{mg}, 0.879 \mathrm{mmol})$, DPPF (1,1'-bis(diphenylphosphino)ferrocene) (487 mg, $0.879 \mathrm{mmol})$ and AgOTF (226 mg, 0.879 $\mathrm{mmol})$. Dichloromethane $(12 \mathrm{~mL})$ was added, and the orange suspension was stirred for $20 \mathrm{~min}$. Filtration through Celite yielded a clear orange solution, which was concentrated to $1 \mathrm{~mL}$ under reduced pressure, layered with $\mathrm{Et}_{2} \mathrm{O}$, and cooled at $-35^{\circ} \mathrm{C}$ for $12 \mathrm{~h}$. The resulting orange crystals were isolated from the supernatant, washed with $2 \times 10 \mathrm{~mL} \mathrm{Et}_{2} \mathrm{O}$, and dried to give $746 \mathrm{mg}$ (90\%) of 9. Single crystals suitable for X-ray diffraction were obtained by allowing $\mathrm{Et}_{2} \mathrm{O}$ to diffuse into a saturated solution of 9 in dichloromethane. ${ }^{1} \mathrm{H}$ NMR $\left(500 \mathrm{MHz}, \mathrm{CD}_{2} \mathrm{Cl}_{2}\right) \delta 2.93(\mathrm{~d}$, $J=9.5 \mathrm{~Hz}, 2 \mathrm{H}), 3.98(\mathrm{q}, J=2.0 \mathrm{~Hz}, 2 \mathrm{H}), 4.35(\mathrm{~s}, 2 \mathrm{H}), 4.46(\mathrm{q}, J=2.0 \mathrm{~Hz}, 2 \mathrm{H}), 4.56(\mathrm{~s}, 2 \mathrm{H})$, $5.34\left(\mathrm{~s}, 1 \mathrm{H}, 0.5 \mathrm{CH}_{2} \mathrm{Cl}_{2}\right), 6.75(\mathrm{~m}, 2 \mathrm{H}), 6.92(\mathrm{~m}, 1 \mathrm{H}), 7.07$ (t, $\left.J=7.3 \mathrm{~Hz}, 2 \mathrm{H}\right), 7.21(\mathrm{~m}, 4 \mathrm{H}), 7.48$ $(\mathrm{m}, 8 \mathrm{H}), 7.57(\mathrm{~m}, 8 \mathrm{H}) ;{ }^{13} \mathrm{C}\left\{{ }^{1} \mathrm{H}\right\}$ NMR $\left(126 \mathrm{MHz}, \mathrm{CD}_{2} \mathrm{Cl}_{2}\right) \delta 54.2\left(\mathrm{CH}_{2} \mathrm{Cl}_{2}\right), 57.5(\mathrm{dd}, J=39.3$, $6.3 \mathrm{~Hz}), 73.5(\mathrm{~d}, J=6.4 \mathrm{~Hz}), 74.2(\mathrm{dd}, J=50.3,2.7 \mathrm{~Hz}), 74.2(\mathrm{~d}, J=7.4 \mathrm{~Hz}), 75.3(\mathrm{~d}, J=9.7$ Hz), 75.4 (dd, $J=55.7,3.5 \mathrm{~Hz}), 76.5(\mathrm{~d}, J=11.9 \mathrm{~Hz}), 118.0$ (dd, $J=9.2,2.8 \mathrm{~Hz}), 121.3$ (t, $J=$ $6.3 \mathrm{~Hz}), 121.5$ (q, $J=321.4 \mathrm{~Hz}), 129.4(\mathrm{~d}, J=9.8 \mathrm{~Hz}), 129.4$ (d, $J=11.8 \mathrm{~Hz}), 130.9$ (d, $J=39.6$ $\mathrm{Hz}), 131.4(\mathrm{~d}, J=2.6 \mathrm{~Hz}), 131.7(\mathrm{~d}, J=3.0 \mathrm{~Hz}), 132.0(\mathrm{q}, J=3.8 \mathrm{~Hz}), 133.1(\mathrm{~d}, J=49.7 \mathrm{~Hz})$, $133.6(\mathrm{~d}, J=12.7 \mathrm{~Hz}), 133.7$ (d, $J=14.8 \mathrm{~Hz}), 133.9$ (dd, 4.3, $2.4 \mathrm{~Hz}) ;{ }^{31} \mathrm{P}\left\{{ }^{1} \mathrm{H}\right\} \mathrm{NMR}(202 \mathrm{MHz}$, $\left.\mathrm{CD}_{2} \mathrm{Cl}_{2}\right) \delta 23.9(\mathrm{~d}, J=48.7 \mathrm{~Hz}), 34.6(\mathrm{~d}, J=45.9 \mathrm{~Hz})$; Anal. Calcd for $\mathrm{C}_{42.5} \mathrm{H}_{36} \mathrm{ClF}_{3} \mathrm{FeO}_{3} \mathrm{P}_{2} \mathrm{PdS}$ : C, 54.10; H, 3.85 Found: C, 54.05; H, 3.97.

Preparation of $\left[\left((\boldsymbol{R})\right.\right.$-Binap)Pd$\left(\boldsymbol{\eta}^{3}\right.$-benzyl) $]$ OTf (10). Into a $20 \mathrm{~mL}$ scintillation vial equipped with a magnetic stirbar was placed $(\mathrm{COD}) \mathrm{Pd}\left(\mathrm{CH}_{2} \mathrm{Ph}\right) \mathrm{Cl}(50.0 \mathrm{mg}, 0.147 \mathrm{mmol})$ and $(R)$-Binap (91.3 $\mathrm{mg}, 0.147 \mathrm{mmol})$. Dichloromethane $(5 \mathrm{~mL})$ was added, and the solution was stirred for 10 min. AgOTF (37.7 $\mathrm{mg}, 0.147 \mathrm{mmol}$ ) was added to the stirring solution, and the resulting suspension was stirred for $10 \mathrm{~min}$. Filtration through Celite yielded a clear solution, which was concentrated to $1 \mathrm{~mL}$ under reduced pressure, layered with $\mathrm{Et}_{2} \mathrm{O}$, and cooled at $-35{ }^{\circ} \mathrm{C}$ for $14 \mathrm{~h}$. The resulting yellow crystals were isolated from the supernatant, washed with $2 \times 5 \mathrm{~mL} \mathrm{Et}_{2} \mathrm{O}$, and dried to give $139 \mathrm{mg}(97 \%)$ of $\mathbf{1 0} .{ }^{1} \mathrm{H}$ NMR $\left(500 \mathrm{MHz}, \mathrm{CD}_{2} \mathrm{Cl}_{2}\right) \delta 2.81(\mathrm{dd}, J=10.0,3.0 \mathrm{~Hz}$, $1 \mathrm{H}), 3.52$ (ddd, $J=9.5,3.5,1.5 \mathrm{~Hz}, 1 \mathrm{H}), 6.43(\mathrm{~d}, J=8.5 \mathrm{~Hz}, 1 \mathrm{H}), 6.62(\mathrm{~d}, J=9.0 \mathrm{~Hz}, 1 \mathrm{H}), 6.69-$ $6.73(\mathrm{~m}, 5 \mathrm{H}) 6.80-6.90(\mathrm{~m}, 5 \mathrm{H}), 6.92(\mathrm{~m}, 1 \mathrm{H}), 6.99(\mathrm{~m}, 1 \mathrm{H}), 7.05-7.11(\mathrm{~m}, 4 \mathrm{H}), 7.14(\mathrm{t}, J=9.0$ $\mathrm{Hz}, 1 \mathrm{H}), 7.24(\mathrm{dd}, J=13.3,7.3 \mathrm{~Hz}, 2 \mathrm{H}), 7.32-7.40(\mathrm{~m}, 2 \mathrm{H}), 7.50-7.80(\mathrm{~m}, 14 \mathrm{H}) ;{ }^{13} \mathrm{C}\left\{{ }^{1} \mathrm{H}\right\} \mathrm{NMR}$ $\left(126 \mathrm{MHz}, \mathrm{CD}_{2} \mathrm{Cl}_{2}\right)$ selected resonances; the core of the aromatic region has many severly 
overlapping resonances $\delta 56.9(\mathrm{dd}, J=41.3,8.5 \mathrm{~Hz}), 117.1$ (broad), $121.5(\mathrm{q}, J=322.5 \mathrm{~Hz})$, $122.3(\mathrm{dd}, J=8.9,5.7 \mathrm{~Hz}), 123.7$ (d, $J=46.9 \mathrm{~Hz}), 125.7(\mathrm{~d}, J=52.2) ;{ }^{31} \mathrm{P}\left\{{ }^{1} \mathrm{H}\right\}$ NMR $(162$ $\left.\mathrm{MHz}, \mathrm{CD}_{2} \mathrm{Cl}_{2}\right) \delta 21.1(\mathrm{~d}, J=57.7 \mathrm{~Hz}), 31.4(\mathrm{~d}, J=57.5 \mathrm{~Hz})$; Anal. Calcd for $\mathrm{C}_{52} \mathrm{H}_{39} \mathrm{~F}_{3} \mathrm{O}_{3} \mathrm{P}_{2} \mathrm{PdS}$ : C, 64.43; H, 4.06 Found: C, 64.46; H, 3.99.

Preparation of $\left[(D P E p h o s) P d\left(\eta^{3}\right.\right.$-allyl $\left.)\right] C l \cdot 0.5 \mathbf{C H}_{2} \mathbf{C l}_{2}$ (11). Into a $20 \mathrm{~mL}$ scintillation vial equipped with a magnetic stirbar was placed $\left[\mathrm{Pd}\left(\eta^{3} \text {-allyl }\right) \mathrm{Cl}\right]_{2}(25.0 \mathrm{mg}, 0.0683 \mathrm{mmol})$ and DPEphos (73.8 mg, $0.137 \mathrm{mmol})$. Benzene $(5 \mathrm{~mL})$ was added, and the solution was stirred for $10 \mathrm{~min}$. All volatile materials were removed in vacuo, and the residue was redissolved in $0.2 \mathrm{~mL}$ dichloromethane. Diffusion of pentane into this solution at $-35^{\circ} \mathrm{C}$ over $12 \mathrm{~h}$ afforded yellow crystals, which were isolated, washed two times with $5 \mathrm{~mL}$ of pentane and dried in vacuo to give $98.1 \mathrm{mg}(94 \%)$ of 11. ${ }^{1} \mathrm{H} \mathrm{NMR}\left(500 \mathrm{MHz}, \mathrm{CD}_{2} \mathrm{Cl}_{2}\right) \delta 3.77$ (br s, 4H), 5.33 (s, 1H, 1/2 $\left.\mathrm{CH}_{2} \mathrm{Cl}_{2}\right)$, $5.81(\mathrm{qt}, J=10.5 \mathrm{~Hz}, 1 \mathrm{H}), 6.75(\mathrm{~m}, 2 \mathrm{H}), 6.92(\mathrm{~m}, 2 \mathrm{H}), 6.98(\mathrm{t}, J=7.5 \mathrm{~Hz}, 2 \mathrm{H}), 7.25-7.50(\mathrm{~m}$, $22 \mathrm{H}) ;{ }^{13} \mathrm{C}\left\{{ }^{1} \mathrm{H}\right\}$ NMR $\left(126 \mathrm{MHz}, \mathrm{CD}_{2} \mathrm{Cl}_{2}\right) \delta 54.2\left(\mathrm{CH}_{2} \mathrm{Cl}_{2}\right), 78.2(\mathrm{t}, J=15.8 \mathrm{~Hz}), 121.0,121.1(\mathrm{t}$, $J=5.3 \mathrm{~Hz}), 122.5(\mathrm{t}, J=22.1 \mathrm{~Hz}), 125.3(\mathrm{t}, J=3.5 \mathrm{~Hz}), 129.3(\mathrm{t}, J=5.3 \mathrm{~Hz}), 131.3,131.5(\mathrm{t}, J=$ $21.8 \mathrm{~Hz}), 133.2,133.7(\mathrm{t}, J=6.8 \mathrm{~Hz}), 134.8,158.7(\mathrm{t}, J=3.8 \mathrm{~Hz}) ;{ }^{31} \mathrm{P}\left\{{ }^{1} \mathrm{H}\right\} \mathrm{NMR}(202 \mathrm{MHz}$, $\mathrm{CD}_{2} \mathrm{Cl}_{2}$ ) $\delta$ 12.9; Anal. Calcd for $\mathrm{C}_{39.5} \mathrm{H}_{34} \mathrm{Cl}_{2} \mathrm{OP}_{2} \mathrm{Pd}$ : C, 62.10; H, 4.49 Found: C, 62.40; H, 4.60.

Preparation of $\left[(D P P F) P d\left(\eta^{3}\right.\right.$-allyl) $] C l \cdot 0.5 \mathbf{C}_{6} \mathbf{H}_{6}$ (12). Into a $50 \mathrm{~mL}$ round bottom flask equipped with a magnetic stirbar was placed $\left[\operatorname{Pd}\left(\eta^{3} \text {-allyl }\right) \mathrm{Cl}\right]_{2}(200 . \mathrm{mg}, 0.547 \mathrm{mmol})$. Into a second $50 \mathrm{~mL}$ round bottom flask equipped with a magnetic stirbar was placed DPPF (604 mg, $1.09 \mathrm{mmol})$. Benzene $(15 \mathrm{~mL})$ was added to each flask, and the resulting solutions stirred for 10 min at $50{ }^{\circ} \mathrm{C}$ to ensure complete dissolution. The solutions were subsequently combined, and an orange-red precipitate immediately began to form. After stirring for $30 \mathrm{~min}$ at $50{ }^{\circ} \mathrm{C}$, the resulting suspension was allowed to cool to room temperature, and the precipitate was isolated by filtration in air. The precipitate was washed two times with $50 \mathrm{~mL}$ of $\mathrm{Et}_{2} \mathrm{O}$ and dried in vacuo to give $789 \mathrm{mg}(93 \%)$ of $\mathbf{1 2}$. Single crystals suitable for X-ray diffraction were obtained by allowing $\mathrm{Et}_{2} \mathrm{O}$ to diffuse into a saturated solution of 12 in dichloromethane. ${ }^{1} \mathrm{H}$ NMR $(500 \mathrm{MHz}$, $\left.\mathrm{CD}_{2} \mathrm{Cl}_{2}\right) \delta 3.88$ (br s, 4H), 4.39 (br s, $\left.4 \mathrm{H}\right), 4.44$ (s, 4H), 5.84 (qt, $\left.J=10.5 \mathrm{~Hz}, 1 \mathrm{H}\right), 7.44$ (t, $J=$ $7.5 \mathrm{~Hz}, 8 \mathrm{H}), 7.53(\mathrm{t}, J=7.3 \mathrm{~Hz}, 4 \mathrm{H}), 7.59(\mathrm{dd}, J=11.8,7.3 \mathrm{~Hz}, 8 \mathrm{H}) ;{ }^{13} \mathrm{C}\left\{{ }^{1} \mathrm{H}\right\} \mathrm{NMR}(126 \mathrm{MHz}$, $\left.\mathrm{CD}_{2} \mathrm{Cl}_{2}\right) \delta 73.7(\mathrm{t}, J=3.3 \mathrm{~Hz}), 75.5(\mathrm{t}, J=29.1 \mathrm{~Hz}), 76.4(\mathrm{t}, J=5.5 \mathrm{~Hz}), 78.2(\mathrm{t}, J=15.6 \mathrm{~Hz})$, $123.5(\mathrm{t}, J=5.5 \mathrm{~Hz}), 129.3(\mathrm{t}, J=5.5 \mathrm{~Hz}), 131.6,133.6(\mathrm{t}, J=22.5 \mathrm{~Hz}), 134.0(\mathrm{t}, J=6.9 \mathrm{~Hz})$; 
${ }^{31} \mathrm{P}\left\{{ }^{1} \mathrm{H}\right\}$ NMR (202 MHz, $\left.\mathrm{CD}_{2} \mathrm{Cl}_{2}\right) \delta$ 24.2; Anal. Calcd for $\mathrm{C}_{40} \mathrm{H}_{36} \mathrm{ClFeP}_{2} \mathrm{Pd}$ : C, 61.88; H, 4.67 Found: C, 61.56; H, 4.48.

Preparation of $\left[((R)-B i n a p) P d\left(\eta^{3}\right.\right.$-allyl $\left.)\right] \mathbf{C l} \cdot \mathbf{C H}_{2} \mathbf{C l}_{\mathbf{2}}$ (13). Into a $20 \mathrm{~mL}$ scintillation vial equipped with a magnetic stirbar was placed $\left[\mathrm{Pd}\left(\eta^{3} \text {-allyl }\right) \mathrm{Cl}\right]_{2}(50.0 \mathrm{mg}, 0.137 \mathrm{mmol})$ and $(R)$ Binap (170. mg, $0.274 \mathrm{mmol})$. Benzene $(5 \mathrm{~mL})$ was added, and the solution was stirred for 10 min. All volatile materials were removed in vacuo and the residue was redissolved in $0.2 \mathrm{~mL}$ dichloromethane. Diffusion of pentane into this solution at $-35^{\circ} \mathrm{C}$ over $12 \mathrm{~h}$ afforded off-white crystals which were isolated, washed two times with $5 \mathrm{~mL}$ of pentane and dried in vacuo to give $241 \mathrm{mg}(99 \%)$ of 13. ${ }^{1} \mathrm{H}$ NMR (500 MHz, $\mathrm{CD}_{2} \mathrm{Cl}_{2}$ ) $\delta 3.97$ (br s, 4H), 5.33 (s, 2H, $\mathrm{CH}_{2} \mathrm{Cl}_{2}$ ), 5.92 (qt, $J=10.5 \mathrm{~Hz}, 1 \mathrm{H}), 6.62(\mathrm{~d}, J=8.5 \mathrm{~Hz}, 2 \mathrm{H}), 6.69$ (t, $J=7.8 \mathrm{~Hz}, 4 \mathrm{H}), 6.84(\mathrm{t}, J=7.5 \mathrm{~Hz}, 2 \mathrm{H})$, $7.07(\mathrm{~m}, 2 \mathrm{H}), 7.25(\mathrm{dd}, J=12.8,6.8 \mathrm{~Hz}, 4 \mathrm{H}), 7.35(\mathrm{~m}, 4 \mathrm{H}), 7.52-7.61(\mathrm{~m}, 10 \mathrm{H}), 7.73(\mathrm{~m}, 4 \mathrm{H})$; ${ }^{13} \mathrm{C}\left\{{ }^{1} \mathrm{H}\right\}$ NMR $\left(126 \mathrm{MHz}, \mathrm{CD}_{2} \mathrm{Cl}_{2}\right) \delta 54.2\left(\mathrm{CH}_{2} \mathrm{Cl}_{2}\right), 77.1,123.0(\mathrm{t}, J=6.0 \mathrm{~Hz}), 125.6(\mathrm{t}, J=24.7$ $\mathrm{Hz}), 127.0,127.2(\mathrm{t}, J=4.8 \mathrm{~Hz}), 127.4,127.8,128.2(\mathrm{t}, J=6.1 \mathrm{~Hz}), 128.4,129.2,129.2(\mathrm{t}, J=$ $5.6 \mathrm{~Hz}), 129.6(\mathrm{t}, J=22.1 \mathrm{~Hz}), 130.6,131.3,131.7(\mathrm{t}, J=22.4 \mathrm{~Hz}), 133.8(\mathrm{t}, J=3.1 \mathrm{~Hz}), 134.1$, 134.2 (t, $J=5.9 \mathrm{~Hz}), 134.9(\mathrm{t}, J=7.4 \mathrm{~Hz}), 139.1(\mathrm{t}, J=7.4 \mathrm{~Hz}) ;{ }^{31} \mathrm{P}\left\{{ }^{1} \mathrm{H}\right\} \mathrm{NMR}(202 \mathrm{MHz}$, $\left.\mathrm{CD}_{2} \mathrm{Cl}_{2}\right) \delta$ 22.6; Anal. Calcd for $\mathrm{C}_{48} \mathrm{H}_{39} \mathrm{Cl}_{3} \mathrm{P}_{2} \mathrm{Pd}$ : C, 64.74; H, 4.41 Found: C, 64.74; H, 4.36 .

Preparation of $\left[(D P P E) P d\left(\eta^{3}\right.\right.$-allyl) $] C l \cdot 0.5 \mathbf{C}_{6} \mathbf{H}_{6}(\mathbf{1 4})$. Into a $50 \mathrm{~mL}$ round bottom flask equipped with a magnetic stirbar was placed $\left[\mathrm{Pd}\left(\eta^{3} \text {-allyl }\right) \mathrm{Cl}\right]_{2}(200 . \mathrm{mg}, 0.547 \mathrm{mmol})$. Into a second $50 \mathrm{~mL}$ round bottom flask equipped with a magnetic stirbar was placed DPPE (434 mg, $1.09 \mathrm{mmol})$. Benzene $(20 \mathrm{~mL})$ was added to each flask, and the resulting solutions were stirred for $10 \mathrm{~min}$ at $50{ }^{\circ} \mathrm{C}$ to ensure complete dissolution. The solutions were subsequently combined, and an off-white precipitate immediately began to form. After stirring for $30 \mathrm{~min}$ at $50{ }^{\circ} \mathrm{C}$, the resulting suspension was allowed to cool to room temperature, and the precipitate was isolated by filtration in air. The precipitate was washed two times with $30 \mathrm{~mL}$ of $\mathrm{Et}_{2} \mathrm{O}$ and dried in vacuo to give $656 \mathrm{mg}(97 \%)$ of $14 .{ }^{1} \mathrm{H} \mathrm{NMR}\left(500 \mathrm{MHz}, \mathrm{CD}_{2} \mathrm{Cl}_{2}\right) \delta 2.73(\mathrm{~d}, J=18.0 \mathrm{~Hz}, 4 \mathrm{H}), 4.16(\mathrm{~m}$, $4 \mathrm{H}), 5.82$ (qt, $J=10.5 \mathrm{~Hz}, 1 \mathrm{H}), 7.45-7.55(\mathrm{~m}, 12 \mathrm{H}), 7.58-7.65(\mathrm{~m}, 8 \mathrm{H}) ;{ }^{13} \mathrm{C}\left\{{ }^{1} \mathrm{H}\right\}$ NMR $(126$ $\left.\mathrm{MHz}, \mathrm{CD}_{2} \mathrm{Cl}_{2}\right) \delta 27.7(\mathrm{t}, J=22.9 \mathrm{~Hz}), 71.3(\mathrm{t}, J=17.0 \mathrm{~Hz}), 123.7(\mathrm{t}, J=6.0 \mathrm{~Hz}), 129.7(\mathrm{t}, J=$ $5.1 \mathrm{~Hz}), 130.4(\mathrm{t}, J=21.1 \mathrm{~Hz}), 132.0,133.1(\mathrm{t}, J=6.4 \mathrm{~Hz}) ;{ }^{31} \mathrm{P}\left\{{ }^{1} \mathrm{H}\right\} \mathrm{NMR}\left(202 \mathrm{MHz}, \mathrm{CD}_{2} \mathrm{Cl}_{2}\right)$

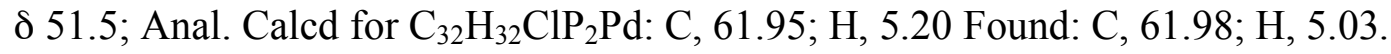

Preparation of $\left[\left(X_{\text {antphos })} \mathbf{P d}\left(\eta^{3}\right.\right.\right.$-allyl) $] \mathbf{B F}_{4}$ (15). Into a $20 \mathrm{~mL}$ scintillation vial equipped with a magnetic stirbar was placed $\left[\mathrm{Pd}\left(\eta^{3} \text {-allyl }\right) \mathrm{Cl}\right]_{2}(50.0 \mathrm{mg}, 0.137 \mathrm{mmol})$ and Xantphos $(159 . \mathrm{mg}$, 
$0.274 \mathrm{mmol})$. Dichloromethane $(10 \mathrm{~mL})$ was added, and the solution was stirred for $5 \mathrm{~min}$. $\mathrm{AgBF}_{4}$ (53.3 mg, $\left.0.274 \mathrm{mmol}\right)$ was added to the stirring solution, and the resulting suspension was stirred for $10 \mathrm{~min}$. Filtration through Celite yielded a clear solution, which was concentrated to $2 \mathrm{~mL}$ under reduced pressure, layered with $\mathrm{Et}_{2} \mathrm{O}$, and cooled at $-35^{\circ} \mathrm{C}$ for $12 \mathrm{~h}$. The resulting white crystals were washed two times with $5 \mathrm{~mL}^{\text {of }} \mathrm{Et}_{2} \mathrm{O}$ and dried in vacuo to give $203 \mathrm{mg}$ (91\%) of 15. ${ }^{1} \mathrm{H}$ NMR $\left(500 \mathrm{MHz}, \mathrm{CD}_{2} \mathrm{Cl}_{2}\right) \delta 1.67(\mathrm{~s}, 6 \mathrm{H}$, these two resonances for the inequivalent methyl groups are resolved at 1.80 and 1.54 in $\mathrm{CDCl}_{3}$, but the aromatic resonances are poorly resolved in $\left.\mathrm{CDCl}_{3}\right), 3.43(\mathrm{~m}, 2 \mathrm{H}), 3.77(\mathrm{~d}, J=7.5 \mathrm{~Hz}, 2 \mathrm{H}), 5.85$ (sept, $J=7.0 \mathrm{~Hz}$, 1H), $6.59(\mathrm{~m}, 2 \mathrm{H}), 7.13(\mathrm{~m}, 8 \mathrm{H}), 7.19$ (t, $J=8.0 \mathrm{~Hz}, 2 \mathrm{H}), 7.35$ (t, $J=7.3 \mathrm{~Hz}, 8 \mathrm{H}), 7.45(\mathrm{t}, J=7.3$ $\mathrm{Hz}, 4 \mathrm{H}), 7.69(\mathrm{dd}, J=8.0,1.0 \mathrm{~Hz}, 2 \mathrm{H}) ;{ }^{13} \mathrm{C}\left\{{ }^{1} \mathrm{H}\right\} \mathrm{NMR}\left(126 \mathrm{MHz}, \mathrm{CD}_{2} \mathrm{Cl}_{2}\right) \delta$ 27.9, 36.5, $79.8(\mathrm{t}$, $J=14.9 \mathrm{~Hz}), 118.0(\mathrm{~m}), 122.4(\mathrm{t}, J=4.9 \mathrm{~Hz}), 125.3(\mathrm{t}, J=3.7 \mathrm{~Hz}), 128.9,129.4(\mathrm{t}, J=5.4 \mathrm{~Hz})$, 131.3, 131.5 (broad), 132.4, $133.1(\mathrm{t}, J=6.2 \mathrm{~Hz}), 134.8,155.5(\mathrm{t}, J=3.9 \mathrm{~Hz}) ;{ }^{31} \mathrm{P}\left\{{ }^{1} \mathrm{H}\right\} \mathrm{NMR}$

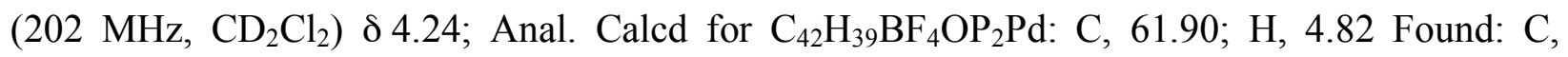
$61.82 ; \mathrm{H}, 4.67$.

Preparation of $\left[\left((\boldsymbol{R})-\mathrm{Binap}_{)}\right) \mathbf{P d}\left(\boldsymbol{\eta}^{\mathbf{3}}\right.\right.$-allyl $\left.)\right] \mathbf{B F}_{\mathbf{4}}$ (16). Into a $20 \mathrm{~mL}$ scintillation vial equipped with a magnetic stirbar was placed $\left[\mathrm{Pd}\left(\eta^{3} \text {-allyl }\right) \mathrm{Cl}\right]_{2}(29.4 \mathrm{mg}, 0.0804 \mathrm{mmol})$ and $(R)$-Binap (100. $\mathrm{mg}, 0.161 \mathrm{mmol})$. Dichloromethane $(4 \mathrm{~mL})$ was added, and the solution was stirred for 10 min. $\mathrm{AgBF}_{4}(31.3 \mathrm{mg}, 0.161 \mathrm{mmol})$ was added to the stirring solution, and the resulting suspension was stirred for $30 \mathrm{~min}$. Filtration through Celite yielded a clear solution, which was concentrated to $1 \mathrm{~mL}$ under reduced pressure, layered with $\mathrm{Et}_{2} \mathrm{O}$, and cooled at $-35^{\circ} \mathrm{C}$ for $12 \mathrm{~h}$. The resulting white crystals were washed two times with $3 \mathrm{~mL}$ of $\mathrm{Et}_{2} \mathrm{O}$ and dried in vacuo to give $124 \mathrm{mg}(90 \%)$ of $16 .{ }^{1} \mathrm{H} \mathrm{NMR}\left(500 \mathrm{MHz}, \mathrm{CD}_{2} \mathrm{Cl}_{2}\right) \delta 2.93(\mathrm{t}, J=11.8 \mathrm{~Hz}, 1 \mathrm{H}), 3.92(\mathrm{t}, J=$ $11.8 \mathrm{~Hz}, 1 \mathrm{H}), 4.03$ (t, $J=6.0 \mathrm{~Hz}, 1 \mathrm{H}), 4.17$ (t, $J=5.8 \mathrm{~Hz}, 1 \mathrm{H}), 5.85$ (sept, $J=7.0 \mathrm{~Hz}, 1 \mathrm{H}), 6.57$ $(\mathrm{d}, J=8.5 \mathrm{~Hz}, 1 \mathrm{H}), 6.68(\mathrm{~d}, J=8.5 \mathrm{~Hz}, 1 \mathrm{H}), 6.72(\mathrm{q}, J=8.0 \mathrm{~Hz}, 4 \mathrm{H}), 6.88(\mathrm{q}, J=7.5 \mathrm{~Hz}, 2 \mathrm{H})$, $7.02-7.08(\mathrm{~m}, 3 \mathrm{H}), 7.12(\mathrm{t}, J=7.8 \mathrm{~Hz}, 1 \mathrm{H}), 7.26(\mathrm{dd}, J=12.8,7.8 \mathrm{~Hz}, 2 \mathrm{H}), 7.30-7.43(\mathrm{~m}, 4 \mathrm{H})$, 7.55-7.65 (m, 14H); ${ }^{13} \mathrm{C}\left\{{ }^{1} \mathrm{H}\right\} \mathrm{NMR}\left(126 \mathrm{MHz}, \mathrm{CD}_{2} \mathrm{Cl}_{2}\right.$, allyl resonances only) $\delta 73.4(\mathrm{dd}, J=$ 29.7, 4.1 Hz), $79.6(\mathrm{dd}, J=27.9,4.1 \mathrm{~Hz}), 123.5(\mathrm{t}, J=6.0 \mathrm{~Hz}) ;{ }^{31} \mathrm{P}\left\{{ }^{1} \mathrm{H}\right\} \mathrm{NMR}(202 \mathrm{MHz}$, $\left.\mathrm{CD}_{2} \mathrm{Cl}_{2}\right) \delta 22.1(\mathrm{~d}, J=51 \mathrm{~Hz}), 23.8\left(\mathrm{~d}, J=51 \mathrm{~Hz}\right.$ ); Anal. Calcd for $\mathrm{C}_{47} \mathrm{H}_{37} \mathrm{BF}_{4} \mathrm{P}_{2} \mathrm{Pd}$ : C, 65.87; H, 4.35 Found: C, 65.83; H, 4.18.

Preparation of $[((\boldsymbol{R})-\operatorname{Binap})) \operatorname{Pd}\left(\eta^{3}\right.$-allyl)]OTf (17). Into a $20 \mathrm{~mL}$ scintillation vial equipped with a magnetic stirbar was placed $\left[\mathrm{Pd}\left(\eta^{3} \text {-allyl }\right) \mathrm{Cl}\right]_{2}(29.4 \mathrm{mg}, 0.0804 \mathrm{mmol})$ and $(R)$-Binap 
(100. $\mathrm{mg}, 0.161 \mathrm{mmol})$. Dichloromethane $(4 \mathrm{~mL})$ was added, and the solution was stirred for 10 min. AgOTf (41.3 mg, $0.161 \mathrm{mmol})$ was added to the stirring solution and the resulting suspension was stirred for $30 \mathrm{~min}$. Filtration through Celite yielded a clear solution, which was concentrated to $1 \mathrm{~mL}$ under reduced pressure, layered with $\mathrm{Et}_{2} \mathrm{O}$, and cooled at $-35^{\circ} \mathrm{C}$ for $12 \mathrm{~h}$. The resulting white crystals were washed two times with $3 \mathrm{~mL}$ of $\mathrm{Et}_{2} \mathrm{O}$ and dried in vacuo to give $105 \mathrm{mg}(71 \%)$ of $17 .{ }^{1} \mathrm{H} \mathrm{NMR}\left(500 \mathrm{MHz}, \mathrm{CD}_{2} \mathrm{Cl}_{2}\right) \delta 2.96(\mathrm{t}, J=11.8 \mathrm{~Hz}, 1 \mathrm{H}), 3.96(\mathrm{dd}, J=$ $13.5,9.5 \mathrm{~Hz}, 1 \mathrm{H}), 4.02(\mathrm{~m}, 1 \mathrm{H}), 4.16$ (t, $J=6.8 \mathrm{~Hz}, 1 \mathrm{H}), 5.86$ (sept, $J=7.0 \mathrm{~Hz}, 1 \mathrm{H}), 6.57$ (d, $J=$ $9.0 \mathrm{~Hz}, 1 \mathrm{H}), 6.67(\mathrm{~d}, J=8.5 \mathrm{~Hz}, 1 \mathrm{H}), 6.72(\mathrm{~m}, 4 \mathrm{H}), 6.88(\mathrm{~m}, 2 \mathrm{H}), 7.02-7.08(\mathrm{~m}, 3 \mathrm{H}), 7.12(\mathrm{t}, J=$ 7.8 Hz, 1H), 7.25-7.43 (m, 6H), 7.55-7.65 (m, 14H); ${ }^{13} \mathrm{C}\left\{{ }^{1} \mathrm{H}\right\} \mathrm{NMR}\left(126 \mathrm{MHz}, \mathrm{CD}_{2} \mathrm{Cl}_{2}\right.$, allyl and triflate resonances only) $\delta 73.5(\mathrm{dd}, J=29.4,3.7 \mathrm{~Hz}), 79.7(\mathrm{dd}, J=27.6,3.7 \mathrm{~Hz}), 121.5(\mathrm{q}, J=$ $322.6 \mathrm{~Hz}), 123.5$ (t, $J=6.0 \mathrm{~Hz}) ;{ }^{31} \mathrm{P}\left\{{ }^{1} \mathrm{H}\right\} \mathrm{NMR}\left(202 \mathrm{MHz}, \mathrm{CD}_{2} \mathrm{Cl}_{2}\right) \delta 22.0(\mathrm{~d}, J=49 \mathrm{~Hz}), 23.7$ (d, $J=49 \mathrm{~Hz}$ ); Anal. Calcd for $\mathrm{C}_{48} \mathrm{H}_{37} \mathrm{~F}_{3} \mathrm{O}_{3} \mathrm{P}_{2} \mathrm{PdS}: \mathrm{C}, 62.72 ; \mathrm{H}, 4.06$ Found: $\mathrm{C}, 62.47 ; \mathrm{H}, 3.86$.

Preparation of [(Xantphos)Pd $\left(\eta^{3}\right.$-benzyl)Br] (18). Into a $20 \mathrm{~mL}$ scintillation vial equipped with a magnetic stirbar was placed (TMEDA)PdBnBr (50.0 mg, $0.127 \mathrm{mmol})$ and Xantphos (73.5 mg, $0.127 \mathrm{mmol})$. Tetrahydrofuran $(5 \mathrm{~mL})$ was added, and the solution was stirred for 10 min. Filtration through Celite yielded a clear solution, which was concentrated to $1 \mathrm{~mL}$ under reduced pressure, layered with pentane, and cooled at $-35{ }^{\circ} \mathrm{C}$ for $12 \mathrm{~h}$. The resulting brown crystals were isolated from the supernatant, washed with $2 \times 10 \mathrm{~mL}$ pentane, and dried to give $73.9 \mathrm{mg}(68 \%)$ of $\mathbf{1 8}$. Single crystals suitable for X-ray diffraction were obtained by allowing pentane to diffuse into a saturated solution of $\mathbf{1 8}$ in tetrahydrofuran. ${ }^{1} \mathrm{H}$ NMR (500 MHz, $\left.\mathrm{CD}_{2} \mathrm{Cl}_{2}\right) \delta 1.72(\mathrm{~s}, 6 \mathrm{H}), 3.27(\mathrm{~s}, 2 \mathrm{H}), 6.38(\mathrm{~d}, J=7.0 \mathrm{~Hz}, 2 \mathrm{H}), 6.5(\mathrm{t}, J=7.8 \mathrm{~Hz}, 2 \mathrm{H}), 6.69(\mathrm{~m}$, 1H), $6.83(\mathrm{~m}, 2 \mathrm{H}), 7.13(\mathrm{t}, J=7.5 \mathrm{~Hz}, 2 \mathrm{H}), 7.20(\mathrm{t}, J=7.8 \mathrm{~Hz}, 8 \mathrm{H}), 7.28(\mathrm{t}, J=7.5 \mathrm{~Hz}, 4 \mathrm{H}), 7.44$ (m, 8H), $7.57(\mathrm{dd}, J=8.0,1.0 \mathrm{~Hz}, 2 \mathrm{H}),{ }^{13} \mathrm{C}\left\{{ }^{1} \mathrm{H}\right\} \mathrm{NMR}\left(126 \mathrm{MHz}, \mathrm{CD}_{2} \mathrm{Cl}_{2}\right) \delta 27.8,36.4,46.8(\mathrm{t}$, $J=21.7 \mathrm{~Hz}), 121.4(\mathrm{t}, J=18.8 \mathrm{~Hz}), 124.1(\mathrm{t}, J=2.9 \mathrm{~Hz}), 125.3,125.9(\mathrm{t}, J=4.2 \mathrm{~Hz}), 126.9$, $128.2(\mathrm{t}, J=3.7 \mathrm{~Hz}), 128.5(\mathrm{t}, J=4.7 \mathrm{~Hz}), 129.1(\mathrm{t}, J=3.3 \mathrm{~Hz}), 130.1,131.1,132.4(\mathrm{t}, J=18.8$ $\mathrm{Hz}), 134.5$ (t, $J=6.3 \mathrm{~Hz}), 134.7,155.1$ (t, $J=4.3 \mathrm{~Hz}) ;{ }^{31} \mathrm{P}\left\{{ }^{1} \mathrm{H}\right\} \mathrm{NMR}\left(202 \mathrm{MHz}, \mathrm{CD}_{2} \mathrm{Cl}_{2}\right)$ $\delta$ 12.6; Anal. Calcd for $\mathrm{C}_{46} \mathrm{H}_{39} \mathrm{BrOP} 2 \mathrm{Pd}: \mathrm{C}, 64.54 ; \mathrm{H}, 4.59$ Found: C, 64.80; H, 4.53.

Preparation of [(Xantphos)Pd( $\boldsymbol{\eta}^{3}$-1,1-dimethylallyl)]OTf (19). Into a $20 \mathrm{~mL}$ scintillation vial equipped with a magnetic stirbar was placed $\left[\operatorname{Pd}\left(\eta^{3}-1,1-\text { dimethylallyl }\right) \mathrm{Cl}\right]_{2}(40.0 \mathrm{mg}, 0.0948$ mmol) and Xantphos (109.7 mg, $0.190 \mathrm{mmol})$. Dichloromethane $(5 \mathrm{~mL})$ was added, and the solution was stirred for $10 \mathrm{~min}$. AgOTf $(48.7 \mathrm{mg}, 0.190 \mathrm{mmol})$ was added to the stirring 
solution, and the resulting suspension was stirred for $30 \mathrm{~min}$. Filtration through Celite yielded a clear solution, which was concentrated to $1 \mathrm{~mL}$ under reduced pressure, layered with $\mathrm{Et}_{2} \mathrm{O}$, and cooled at $-35^{\circ} \mathrm{C}$ for $12 \mathrm{~h}$. The resulting off-white precipitate was washed two times with $3 \mathrm{~mL}$ of $\mathrm{Et}_{2} \mathrm{O}$ and dried in vacuo to give $158 \mathrm{mg}(92 \%)$ of $19 .{ }^{1} \mathrm{H} \mathrm{NMR}\left(500 \mathrm{MHz}, \mathrm{CD}_{2} \mathrm{Cl}_{2}\right) \delta 0.92(\mathrm{t}, J=$ $8.8 \mathrm{~Hz}, 3 \mathrm{H}), 1.04$ (t, $J=5.5 \mathrm{~Hz}, 3 \mathrm{H}), 1.48$ (br s, 3H), 1.86 (br s, 3H), 2.76 (br s, 1H), 3.41 (br s, $1 \mathrm{H}), 5.45$ (t, $J=10.3 \mathrm{~Hz}, 1 \mathrm{H}), 6.72(\mathrm{t}, J=8.3 \mathrm{~Hz}, 2 \mathrm{H}), 7.16(\mathrm{br} \mathrm{s}, 10 \mathrm{H}), 7.21$ (td, $J=7.8,1.5 \mathrm{~Hz}$, 2H), 7.44 (br s, 10H), $7.68(\mathrm{~d}, J=7.5 \mathrm{~Hz}, 2 \mathrm{H}) ;{ }^{13} \mathrm{C}\left\{{ }^{1} \mathrm{H}\right\} \mathrm{NMR}\left(126 \mathrm{MHz}, \mathrm{CD}_{2} \mathrm{Cl}_{2}\right) \delta 21.2(\mathrm{t}, J=$ $2.1 \mathrm{~Hz}), 24.2(\mathrm{br}), 26.2(\mathrm{t}, J=2.8 \mathrm{~Hz}), 31.2(\mathrm{br}), 36.5,62.8(\mathrm{t}, J=16.0 \mathrm{~Hz}), 111.6(\mathrm{t}, J=5.5 \mathrm{~Hz})$, $118.9(\mathrm{~d}, J=38.3 \mathrm{~Hz}), 121.4(\mathrm{q}, J=322.4 \mathrm{~Hz}), 125.3(\mathrm{~d}, J=6.0 \mathrm{~Hz}), 128.2(\mathrm{t}, J=13.2 \mathrm{~Hz})$, 128.6, 129.3 (d, $J=10.8 \mathrm{~Hz}), 131.2,132.1,133.3$ (br), 134.9 (d, $J=3.7 \mathrm{~Hz}), 155.7$ (d, $J=8.2$ $\mathrm{Hz}$ ) (note: one ipso carbon resonance could not be located and is believed to lie under the broad resonance at 133.3, based on chemical shifts of the related Xantphos complexes in this work); ${ }^{31} \mathrm{P}\left\{{ }^{1} \mathrm{H}\right\}$ NMR (202 MHz, $\mathrm{CD}_{2} \mathrm{Cl}_{2}$ ) $\delta 5.53$ (br s), 10.8 (br, s); Anal. Calcd for $\mathrm{C}_{45} \mathrm{H}_{41} \mathrm{~F}_{3} \mathrm{O}_{4} \mathrm{P}_{2} \mathrm{PdS}$ : C, 59.84; H, 4.58 Found: C, 59.74; H, 4.58.

Preparation of [(DPEphos)Pd( $\boldsymbol{\eta}^{3}$-1,1-dimethylallyl)]OTf (20). Into a $20 \mathrm{~mL}$ scintillation vial equipped with a magnetic stirbar was placed $\left[\operatorname{Pd}\left(\eta^{3}-1,1-\text { dimethylallyl }\right) \mathrm{Cl}\right]_{2}(40.0 \mathrm{mg}, 0.0948$ mmol) and DPEphos (102 $\mathrm{mg}, 0.190 \mathrm{mmol})$. Dichloromethane $(5 \mathrm{~mL})$ was added, and the solution was stirred for $10 \mathrm{~min}$. AgOTf $(48.7 \mathrm{mg}, 0.190 \mathrm{mmol})$ was added to the stirring solution, and the resulting suspension was stirred for $30 \mathrm{~min}$. Filtration through Celite yielded a clear solution, which was concentrated to $1 \mathrm{~mL}$ under reduced pressure, layered with $\mathrm{Et}_{2} \mathrm{O}$, and cooled at $-35^{\circ} \mathrm{C}$ for $12 \mathrm{~h}$. The resulting orange crystals were washed two times with $5 \mathrm{~mL}$ of $\mathrm{Et}_{2} \mathrm{O}$ and dried in vacuo to give $151 \mathrm{mg}(93 \%)$ of $20 .{ }^{1} \mathrm{H}$ NMR $\left(500 \mathrm{MHz}, \mathrm{CD}_{2} \mathrm{Cl}_{2}\right) \delta 1.04(\mathrm{t}, J=$ $5.8 \mathrm{~Hz}, 3 \mathrm{H}), 1.26(\mathrm{dd}, J=11.5,6.0 \mathrm{~Hz}, 3 \mathrm{H}), 2.85(\mathrm{ddd}, J=12.8,10.5,2.3 \mathrm{~Hz}, 1 \mathrm{H}), 3.61(\mathrm{dt}, J=$ $7.5,2.5 \mathrm{~Hz}, 1 \mathrm{H}), 5.40(\mathrm{dd}, J=13.8,7.8 \mathrm{~Hz}, 1 \mathrm{H}), 6.71(\mathrm{dd}, J=7.8,4.8 \mathrm{~Hz}, 1 \mathrm{H}), 6.81(\mathrm{dt}, J=8.5$, $1.5 \mathrm{~Hz}, 1 \mathrm{H}), 6.86(\mathrm{t}, J=7.5,1 \mathrm{H}), 6.99-7.06(\mathrm{~m}, 2 \mathrm{H}), 7.15-7.60(\mathrm{~m}, 23 \mathrm{H}) ;{ }^{13} \mathrm{C}\left\{{ }^{1} \mathrm{H}\right\}$ NMR $(126$ $\mathrm{MHz}, \mathrm{CD}_{2} \mathrm{Cl}_{2}$ ) ( 3-1,1-dimethylallyl and triflate resonances) $\delta 21.3(\mathrm{~d}, J=4.7 \mathrm{~Hz}), 26.4(\mathrm{~d}, J=$ $3.5 \mathrm{~Hz}), 65.6(\mathrm{~d}, J=29.9 \mathrm{~Hz}), 113.3(\mathrm{t}, J=5.7 \mathrm{~Hz}), 119.0,121.5(\mathrm{q}, J=322.1 \mathrm{~Hz}) ;{ }^{31} \mathrm{P}\left\{{ }^{1} \mathrm{H}\right\}$ NMR (202 MHz, $\left.\mathrm{CD}_{2} \mathrm{Cl}_{2}\right) \delta 13.0(\mathrm{~d}, J=41.8 \mathrm{~Hz}), 14.8(\mathrm{~d}, J=41.4 \mathrm{~Hz})$; Anal. Calcd for $\mathrm{C}_{42} \mathrm{H}_{37} \mathrm{~F}_{3} \mathrm{O}_{4} \mathrm{P}_{2} \mathrm{PdS}$ : C, 58.44; $\mathrm{H}, 4.32$ Found: $\mathrm{C}, 58.22 ; \mathrm{H}, 4.57$.

Preparation of [(DPPF)Pd( $\boldsymbol{\eta}^{3}$-1,1-dimethylallyl)]OTf $\cdot \mathbf{C H}_{\mathbf{2}} \mathbf{C l}_{\mathbf{2}}$ (21). Into a $20 \mathrm{~mL}$ scintillation vial equipped with a magnetic stirbar was placed $\left[\operatorname{Pd}\left(\eta^{3}-1,1-\text { dimethylallyl }\right) \mathrm{Cl}\right]_{2}(40.0 \mathrm{mg}, 0.0948$ 
mmol) and DPPF (105.1 $\mathrm{mg}, 0.190 \mathrm{mmol})$. Dichloromethane $(5 \mathrm{~mL})$ was added, and the solution was stirred for $10 \mathrm{~min}$. AgOTf $(48.7 \mathrm{mg}, 0.190 \mathrm{mmol})$ was added to the stirring solution, and the resulting suspension was stirred for $30 \mathrm{~min}$. Filtration through Celite yielded a clear solution, which was concentrated to $1 \mathrm{~mL}$ under reduced pressure, layered with $\mathrm{Et}_{2} \mathrm{O}$, and cooled at $-35{ }^{\circ} \mathrm{C}$ for $12 \mathrm{~h}$. The resulting orange crystals were washed two times with $3 \mathrm{~mL}$ of $\mathrm{Et}_{2} \mathrm{O}$ and dried in vacuo to give $156 \mathrm{mg}(93 \%)$ of $21 .{ }^{1} \mathrm{H} \mathrm{NMR}\left(500 \mathrm{MHz}, \mathrm{CD}_{2} \mathrm{Cl}_{2}\right) \delta 1.11(\mathrm{t}, J=$ $6.0 \mathrm{~Hz}, 3 \mathrm{H}), 1.14(\mathrm{dd}, J=11.0,6.5 \mathrm{~Hz}, 3 \mathrm{H}), 2.85(\mathrm{ddd}, J=12.1,11.0,2.8 \mathrm{~Hz}, 1 \mathrm{H}), 3.65(\mathrm{dt}, J=$ 7.5, $2.5 \mathrm{~Hz}, 1 \mathrm{H}$ ), 3.96 (br s, 1H), 4.24 (br s, 1H), 4.28 (br s, 1H), 4.42 (br s, 1H), 4.48 (br s, 1H), $4.51($ br s, 1H), $4.55($ br s, 1H), $4.60($ br s, $1 \mathrm{H}), 5.34(\mathrm{dd}, J=13.0,8.5 \mathrm{~Hz}, 1 \mathrm{H}), 7.42-7.63(\mathrm{~m}$, $20 \mathrm{H}) ;{ }^{13} \mathrm{C}\left\{{ }^{1} \mathrm{H}\right\} \mathrm{NMR}\left(126 \mathrm{MHz}, \mathrm{CD}_{2} \mathrm{Cl}_{2}\right) \delta 21.0(\mathrm{~d}, J=6.0 \mathrm{~Hz}), 25.4(\mathrm{~d}, J=3.8 \mathrm{~Hz}), 65.3(\mathrm{~d}, J=$ $29.9 \mathrm{~Hz}$ ), 73.6-77.2 (broad Cp resonances, 10C), $113.8(\mathrm{t}, J=5.8 \mathrm{~Hz}), 116.4(\mathrm{~d}, J=23.1 \mathrm{~Hz})$, 121.5 (q, $J=321.6 \mathrm{~Hz}), 129.4$ (d, $J=9.5 \mathrm{~Hz}), 129.6$ (d, $J=10.3 \mathrm{~Hz}), 129.6(\mathrm{~d}, J=9.5 \mathrm{~Hz}, 2 \mathrm{C})$, 130.5 (d, $J=43.2 \mathrm{~Hz}), 131.0$ (d, $J=40.2 \mathrm{~Hz}), 131.7,131.8,132.3$ (d, $J=32.0 \mathrm{~Hz}), 132.3$ (d, $J=$ $30.6 \mathrm{~Hz}), 132.7$ (d, $J=45.4 \mathrm{~Hz}), 132.8(\mathrm{~d}, J=12.6 \mathrm{~Hz}), 133.1$ (d, $J=12.6 \mathrm{~Hz}), 133.7$ (d, $J=$ $44.2 \mathrm{~Hz}), 134.4(\mathrm{~d}, J=13.6 \mathrm{~Hz}), 135.1(\mathrm{~d}, J=14.4 \mathrm{~Hz}) ;{ }^{31} \mathrm{P}\left\{{ }^{1} \mathrm{H}\right\}$ NMR $\left(202 \mathrm{MHz}, \mathrm{CD}_{2} \mathrm{Cl}_{2}\right)$

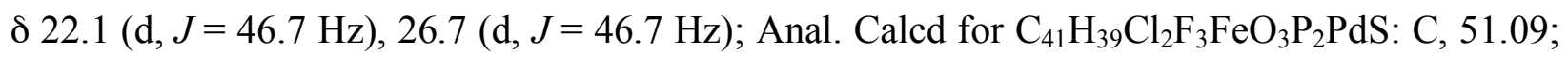
H, 4.08 Found: C, 51.11; H, 3.96.

Preparation of $\left[\left((\boldsymbol{R})\right.\right.$-Binap)Pd$\left(\eta^{3}\right.$-1,1-dimethylallyl $\left.)\right]$ OTf (22). Into a $20 \mathrm{~mL}$ scintillation vial equipped with a magnetic stirbar was placed $\left[\operatorname{Pd}\left(\eta^{3}-1,1-\text { dimethylallyl }\right) \mathrm{Cl}\right]_{2}(40.0 \mathrm{mg}, 0.0948$ $\mathrm{mmol})$ and $R$-Binap (118.0 $\mathrm{mg}, 0.190 \mathrm{mmol})$. Dichloromethane $(5 \mathrm{~mL})$ was added, and the solution was stirred for $10 \mathrm{~min}$. AgOTf $(48.7 \mathrm{mg}, 0.190 \mathrm{mmol}$ ) was added to the stirring solution, and the resulting suspension was stirred for $30 \mathrm{~min}$. Filtration through Celite yielded a clear solution, which was concentrated to $1 \mathrm{~mL}$ under reduced pressure, layered with $\mathrm{Et}_{2} \mathrm{O}$, and cooled at $-35{ }^{\circ} \mathrm{C}$ for $12 \mathrm{~h}$. The resulting white crystals were washed two times with $5 \mathrm{~mL}$ of pentane and dried in vacuo to give $147 \mathrm{mg}(88 \%)$ of $\mathbf{2 2}$. In solution, 22 exists as a pair of diastereomers in a ratio of 5:4. ${ }^{1} \mathrm{H}$ NMR $\left(500 \mathrm{MHz}, \mathrm{CD}_{2} \mathrm{Cl}_{2}\right)$ (major diastereomer, $\eta^{3}-1,1$ dimethylallyl resonances) $\delta 1.03(\mathrm{dd}, J=10.8,6.3 \mathrm{~Hz}, 3 \mathrm{H}), 1.60(\mathrm{t}, J=5.5 \mathrm{~Hz}, 3 \mathrm{H}), 2.29$ (ddd, $J$ $=12.5,11.0,3.0 \mathrm{~Hz}, 1 \mathrm{H}), 3.62(\mathrm{tdd}, J=8.0,3.3,1.5 \mathrm{~Hz}, 1 \mathrm{H}), 5.22(\mathrm{dd}, J=13.0,8.0 \mathrm{~Hz}, 1 \mathrm{H})$, (minor diastereomer, $\eta^{3}$-1,1-dimethylallyl resonances) $\delta 1.00(\mathrm{dd}, J=7.0,5.5 \mathrm{~Hz}, 3 \mathrm{H}), 1.21(\mathrm{dd}$, $J=11.3,7.3 \mathrm{~Hz}, 3 \mathrm{H}), 3.72(\mathrm{~m}, 1 \mathrm{H}), 3.86(\mathrm{td}, J=7.5,2.0 \mathrm{~Hz}, 1 \mathrm{H}), 5.51(\mathrm{dd}, J=13.5,8.0 \mathrm{~Hz}$, 1H), (combined aromatic resonances) $\delta 6.29(\mathrm{~d}, J=8.5 \mathrm{~Hz}, 0.8 \mathrm{H}), 6.39(\mathrm{~d}, J=8.5 \mathrm{~Hz}, 1 \mathrm{H}), 6.47$ 
$(\mathrm{d}, J=8.5 \mathrm{~Hz}, 1 \mathrm{H}), 6.61(\mathrm{~d}, J=7.0 \mathrm{~Hz}, 1.6 \mathrm{H}), 6.70-7.85(\mathrm{~m}, 57.6 \mathrm{H}) ;{ }^{13} \mathrm{C}\left\{{ }^{1} \mathrm{H}\right\} \mathrm{NMR}(126 \mathrm{MHz}$, $\left.\mathrm{CD}_{2} \mathrm{Cl}_{2}\right) \delta\left(\eta^{3}\right.$-1,1-dimethylallyl resonances of both diastereomers and triflate resonances) 20.7 $(\mathrm{d}, J=4.7 \mathrm{~Hz}), 22.8(\mathrm{~d}, J=5.8 \mathrm{~Hz}), 26.0(\mathrm{~d}, J=3.7 \mathrm{~Hz}), 26.3(\mathrm{~d}, J=4.9 \mathrm{~Hz}), 59.6(\mathrm{dd}, J=32.1$, $4.7 \mathrm{~Hz}), 64.0(\mathrm{~d}, J=28.7 \mathrm{~Hz}), 112.9$ (t, $J=6.4 \mathrm{~Hz}), 114.0(\mathrm{~d}, J=4.5 \mathrm{~Hz}), 114.2$ (d, $J=4.4 \mathrm{~Hz})$, $114.6(\mathrm{t}, J=6.4 \mathrm{~Hz}), 121.4(\mathrm{q}, J=312.4 \mathrm{~Hz}) ;{ }^{31} \mathrm{P}\left\{{ }^{1} \mathrm{H}\right\}$ NMR (202 MHz, $\mathrm{CD}_{2} \mathrm{Cl}_{2}$ ) (major diastereomer) $\delta 18.6(\mathrm{~d}, J=54.3 \mathrm{~Hz}), 27.2(\mathrm{~d}, J=56.6 \mathrm{~Hz})$, (minor diastereomer) $\delta 19.0(\mathrm{~d}, J=$ $51.7 \mathrm{~Hz}$ ), 30.8 (d, $J=51.7 \mathrm{~Hz}$ ); Anal. Calcd for $\mathrm{C}_{50} \mathrm{H}_{41} \mathrm{~F}_{3} \mathrm{O}_{3} \mathrm{P}_{2} \mathrm{PdS}$ : C, 63.40; H, 4.36 Found: C, $63.15 ; \mathrm{H}, 4.08$.

Preparation of $\left[(\mathrm{Xantphos}) \mathrm{Pd}\left(\eta^{3}\right.\right.$-cyclohexenyl)Cl] $\cdot \mathbf{0 . 5} \mathbf{C H}_{2} \mathrm{Cl}_{2}$ (23). Into a $20 \mathrm{~mL}$ scintillation vial equipped with a magnetic stirbar was placed $\left[\mathrm{Pd}\left(\eta^{3} \text {-cyclohexenyl }\right) \mathrm{Cl}\right]_{2}(50.0$ $\mathrm{mg}, 0.112 \mathrm{mmol})$ and Xantphos (130. $\mathrm{mg}, 0.224 \mathrm{mmol})$. Benzene $(5 \mathrm{~mL})$ was added, and the solution was stirred for $10 \mathrm{~min}$. All volatile materials were removed in vacuo, and the residue was redissolved in $0.2 \mathrm{~mL}$ dichloromethane. This solution was layered with pentane and cooled to $-35^{\circ} \mathrm{C}$ for $16 \mathrm{~h}$ to afford brown crystals which were isolated, washed two times with $5 \mathrm{~mL}$ of pentane, and dried in vacuo to give $68.6 \mathrm{mg}(38 \%)$ of $23 .{ }^{1} \mathrm{H}$ NMR $\left(500 \mathrm{MHz}, \mathrm{CD}_{2} \mathrm{Cl}_{2}\right) \delta 0.88$ $(\mathrm{m}, 1 \mathrm{H}), 1.29(\mathrm{~m}, 1 \mathrm{H}), 1.44(\mathrm{~m}, 2 \mathrm{H}), 1.56(\mathrm{~m}, 2 \mathrm{H}), 1.65(\mathrm{~s}, 6 \mathrm{H}), 4.90(\mathrm{t}, J=5.5 \mathrm{~Hz}, 2 \mathrm{H}), 5.33(\mathrm{~s}$, $\left.1 \mathrm{H}, \mathrm{CH}_{2} \mathrm{Cl}_{2}\right), 5.95(\mathrm{t}, J=7.0 \mathrm{~Hz}, 1 \mathrm{H}), 6.83(\mathrm{~m}, 2 \mathrm{H}), 7.22(\mathrm{~m}, 10 \mathrm{H}), 7.33(\mathrm{t}, J=7.0 \mathrm{~Hz}), 7.42(\mathrm{t}, J$ $=7.3 \mathrm{~Hz}, 4 \mathrm{H}), 7.66(\mathrm{dd}, J=7.8,1.8 \mathrm{~Hz}, 2 \mathrm{H}) ;{ }^{13} \mathrm{C}\left\{{ }^{1} \mathrm{H}\right\} \mathrm{NMR}\left(126 \mathrm{MHz}, \mathrm{CD}_{2} \mathrm{Cl}_{2}\right) \delta 20.0,28.3$, $28.9(\mathrm{t}, J=2.8 \mathrm{~Hz}), 36.4,54.2\left(\mathrm{CH}_{2} \mathrm{Cl}_{2}\right), 93.6(\mathrm{t}, J=16.0 \mathrm{~Hz}), 111.1(\mathrm{t}, J=5.6 \mathrm{~Hz}), 119.0(\mathrm{~m})$, $125.0(\mathrm{~d}, J=3.3 \mathrm{~Hz}), 128.5,129.2(\mathrm{t}, J=4.9 \mathrm{~Hz}), 130.9,131.8(\mathrm{t}, J=18.8 \mathrm{~Hz}), 132.2,133.3(\mathrm{t}, J$ $=6.4 \mathrm{~Hz}), 134.3(\mathrm{t}, J=1.7 \mathrm{~Hz}), 155.4(\mathrm{t}, J=4.3 \mathrm{~Hz}) ;{ }^{31} \mathrm{P}\left\{{ }^{1} \mathrm{H}\right\} \mathrm{NMR}\left(202 \mathrm{MHz}, \mathrm{CD}_{2} \mathrm{Cl}_{2}\right) \delta 5.22$; Anal. Calcd for $\mathrm{C}_{45.5} \mathrm{H}_{42} \mathrm{Cl}_{2} \mathrm{OP}_{2} \mathrm{Pd}$ : C, 64.74; H, 5.02 Found: C, 64.84; H, 4.63.

Preparation of $\left[(X a n t p h o s) P \mathbf{d C l}_{2}\right] \cdot \mathbf{C H}_{2} \mathbf{C l}_{\mathbf{2}}$ (24). Into a $20 \mathrm{~mL}$ scintillation vial equipped with a magnetic stirbar was placed $\mathrm{Pd}(\mathrm{NCMe})_{2} \mathrm{Cl}_{2}(100 \mathrm{mg}, 0.386 \mathrm{mmol})$ and Xantphos $(223.1 \mathrm{mg}$, $0.386 \mathrm{mmol})$. Dichloromethane $(5 \mathrm{~mL})$ was added, and the solution was stirred for $10 \mathrm{~min}$. Dichloromethane $(5 \mathrm{~mL})$ was added, and the solution was stirred for $10 \mathrm{~min}$. Filtration through Celite yielded a clear yellow solution, which was concentrated to $1 \mathrm{~mL}$ under reduced pressure, layered with $\mathrm{Et}_{2} \mathrm{O}$, and cooled at $-35^{\circ} \mathrm{C}$ for $12 \mathrm{~h}$. The resulting yellow crystals were washed two times with $10 \mathrm{~mL}$ of $\mathrm{Et}_{2} \mathrm{O}$ and dried in vacuo to give $305 \mathrm{mg}$ (94\%) of 24. Single crystals suitable for X-ray diffraction were obtained by allowing $\mathrm{Et}_{2} \mathrm{O}$ to diffuse into a saturated solution of 24 in dichloromethane. ${ }^{1} \mathrm{H}$ NMR $\left(500 \mathrm{MHz}, \mathrm{CD}_{2} \mathrm{Cl}_{2}\right) \delta 1.87(\mathrm{~s}, 6 \mathrm{H}), 5.33\left(\mathrm{~s}, 2 \mathrm{H}, \mathrm{CH}_{2} \mathrm{Cl}_{2}\right)$, 
$7.07(\mathrm{t}, J=6.5 \mathrm{~Hz}, 8 \mathrm{H}), 7.21(\mathrm{t}, J=7.5 \mathrm{~Hz}, 4 \mathrm{H}), 7.31(\mathrm{td}, J=7.8 \mathrm{~Hz}, 2.0 \mathrm{~Hz}, 2 \mathrm{H}), 7.36$ (t, $J=$ $8.8 \mathrm{~Hz}, 8 \mathrm{H}), 7.45(\mathrm{~m}, 2 \mathrm{H}), 7.74(\mathrm{~d}, J=7.5 \mathrm{~Hz}, 2 \mathrm{H}) ;{ }^{13} \mathrm{C}\left\{{ }^{1} \mathrm{H}\right\} \mathrm{NMR}\left(126 \mathrm{MHz}, \mathrm{CD}_{2} \mathrm{Cl}_{2}\right) \delta 26.8$, 37.4, $54.2\left(\mathrm{CH}_{2} \mathrm{Cl}_{2}\right), 120.2$ (d, $\left.J=56.8 \mathrm{~Hz}\right), 125.4$ (t, $\left.J=3.8 \mathrm{~Hz}\right), 128.4$ (d, $\left.J=15.6 \mathrm{~Hz}\right), 128.5$ (d, $J=11.6 \mathrm{~Hz}), 129.9$ (d, $J=55.7 \mathrm{~Hz}), 130.1,130.6,135.0$ (d, $J=10.6 \mathrm{~Hz}), 136.0,154.9$ (t, $J=$ $2.5 \mathrm{~Hz}) ;{ }^{31} \mathrm{P}\left\{{ }^{1} \mathrm{H}\right\} \mathrm{NMR}\left(202 \mathrm{MHz}, \mathrm{CD}_{2} \mathrm{Cl}_{2}\right) \delta$ 22.4; Anal. Calcd for $\mathrm{C}_{40} \mathrm{H}_{34} \mathrm{Cl}_{4} \mathrm{OP}_{2} \mathrm{Pd}$ : C, 57.13; H, 4.08 Found: C, 56.77; H, 3.97 .

Preparation of (COD) $\mathbf{P d}\left(\mathrm{CH}_{2} \mathbf{P h}\right) \mathbf{C l}$. This preparation is an alternative to the published route. ${ }^{6}$ Into a $100 \mathrm{~mL}$ round bottom flask equipped with a magnetic stirbar was placed (COD) $\mathrm{PdCl}_{2}$ $(1.65 \mathrm{~g}, 5.78 \mathrm{mmol})$. THF $(50 \mathrm{~mL})$ was added, and the suspension was cooled to $-78{ }^{\circ} \mathrm{C}$. Benzylmagnesium chloride $(2.90 \mathrm{~mL}, 5.80 \mathrm{mmol})$ was added dropwise, and the resulting suspension was stirred for $3 \mathrm{~h}$. After allowing the dark solution to warm to room temperature slowly, all volatile materials were removed under reduced pressure. The residual solid was dissolved in $25 \mathrm{~mL} \mathrm{CH}_{2} \mathrm{Cl}_{2}$ and washed with $2 \times 25 \mathrm{~mL} \mathrm{H}_{2} \mathrm{O}$. The organic layer was separated and dried over $\mathrm{MgSO}_{4}$ and filtered. The $\mathrm{CH}_{2} \mathrm{Cl}_{2}$ was removed by rotary evaporation to yield a yellow solid which was extracted into $35 \mathrm{~mL}$ of hot acetone and filtered through Celite. The acetone was removed by rotary evaporation yielding a yellow solid, which was recrystallized from $\mathrm{CH}_{2} \mathrm{Cl}_{2} / \mathrm{Et}_{2} \mathrm{O}$ to give $1.42 \mathrm{~g}(72 \%)$ of 5. ${ }^{1} \mathrm{H}$ NMR (400 MHz, $\left.\mathrm{CD}_{2} \mathrm{Cl}_{2}\right) \delta 2.51(\mathrm{~m}, 8 \mathrm{H}), 3.49$ (s, 2H), 4.95 (br s, 2H), 5.83 (br s, 2H), $7.16(\mathrm{~m}, 3 \mathrm{H}), 7.44(\mathrm{dd}, J=6.8,2.0 \mathrm{~Hz}, 2 \mathrm{H}) ;{ }^{13} \mathrm{C}\left\{{ }^{1} \mathrm{H}\right\}$ NMR (101 MHz, $\left.\mathrm{CD}_{2} \mathrm{Cl}_{2}\right) \delta$ 29.1, 32.5, 36.4, 106.0, 125.4, 127.0, 130.0, 131.1, 145.8.

In situ Preparation of [(bisphosphine)Pd( $\eta^{2}$-vinylarene)]. Into a $4 \mathrm{~mL}$ scintillation vial equipped with a magnetic stirbar was placed $\operatorname{CpPd}\left(\eta^{3}\right.$-allyl $)(11.0 \mathrm{mg}, 0.0517 \mathrm{mmol})$ and vinylarene (styrene: $300 \mu \mathrm{L}, 2.62 \mathrm{mmol}, 50$ equiv or 3-trifluoromethylstyrene: $7.7 \mu \mathrm{L}, 0.052$ mmol, 1 equiv). THF $(0.3 \mathrm{~mL})$ was added, and the solution stirred for $5 \mathrm{~min} .1$ equiv of bisphosphine (Xantphos: $30.0 \mathrm{mg}, 0.0518 \mathrm{mmol}$ or DPPF: $28.7 \mathrm{mg}, 0.0518 \mathrm{mmol}$ ) was weighed into a separate $4 \mathrm{~mL}$ scintillation vial and dissolved in THF $(0.3 \mathrm{~mL})$. The two solutions were combined, transferred into a screw-capped NMR tube and were characterized by ${ }^{31} \mathrm{P}$ NMR spectroscopy. The spectra are shown below in figure S3: 

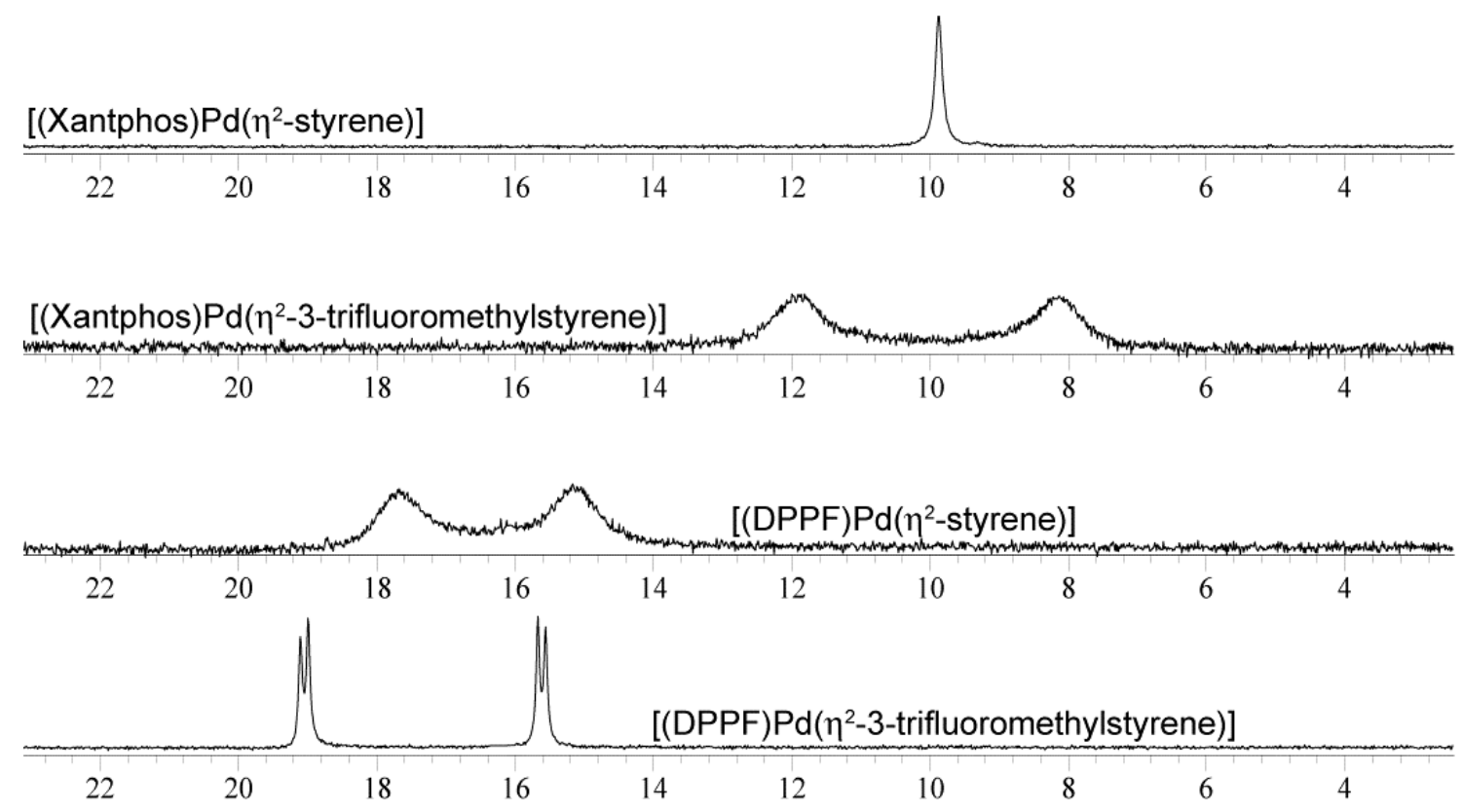

Figure S3: $162 \mathrm{MHz}^{31} \mathrm{P}$ NMR spectra of (bisphosphine)Pd( $\eta^{2}$-vinylarene) in THF.

General Procedure for the Hydroamination of 1,3-Cyclohexadiene with Functionalized Anilines (Table 3). In a drybox, XantphosPd( $\eta^{3}$-allyl)Cl (1) $\left(15.2 \mathrm{mg}, 2.00 \times 10^{-2} \mathrm{mmol}\right), 1,3-$ cyclohexadiene $(191 \mu \mathrm{L}, 2.00 \mathrm{mmol})$, and the aniline $(1.00 \mathrm{mmol})$ were placed into a small vial, dissolved in $2.00 \mathrm{~mL}$ of tetrahydrofuran and sealed with a cap containing a PTFE septum. The reaction mixture was stirred at $25{ }^{\circ} \mathrm{C}$ for $24 \mathrm{~h}$. Upon completion, the reaction mixture was directly adsorbed onto silica gel and purified by flash column chromatography.

$N$-(Cyclohex-2-enyl)-3-nitrobenzenamine (Table 3, Entry 1) The general procedure was followed with 3-nitrobenzenamine $(138 \mathrm{mg}, 1.00 \mathrm{mmol})$. The reaction mixture was purified by flash column chromatography to give $209 \mathrm{mg}$ (96\%) of the hydroamination product: ${ }^{1} \mathrm{H}$ NMR (500 MHz, $\left.\mathrm{CDCl}_{3}, \mathrm{TMS}\right) \delta$ 1.58-1.73 (m, 3H), $1.90(\mathrm{~m}, 1 \mathrm{H}), 2.02(\mathrm{~m}, 2 \mathrm{H}), 3.99(\mathrm{~s}, 1 \mathrm{H}), 4.10(\mathrm{~s}$, $1 \mathrm{H}), 5.68(\mathrm{~m}, 1 \mathrm{H}), 5.87(\mathrm{~m}, 1 \mathrm{H}), 6.85(\mathrm{ddd}, J=8.5,2.5,2.0 \mathrm{~Hz}, 1 \mathrm{H}), 7.22(\mathrm{dd}, J=8.5,8.0 \mathrm{~Hz}$, $1 \mathrm{H}), 7.36(\mathrm{dd}, J=2.5,2.0 \mathrm{~Hz}, 1 \mathrm{H}), 7.44(\mathrm{ddd}, J=8.0,2.5,2.0 \mathrm{~Hz}, 1 \mathrm{H}) ;{ }^{13} \mathrm{C}\left\{{ }^{1} \mathrm{H}\right\} \mathrm{NMR}(126$ $\left.\mathrm{MHz}, \mathrm{CDCl}_{3}\right) \delta 19.3,24.8,28.3,47.7,106.3,111.2,118.9,127.1,129.5,130.8,147.9,149.2$; Anal. Calcd for $\mathrm{C}_{12} \mathrm{H}_{14} \mathrm{~N}_{2} \mathrm{O}_{2}$ : C, 66.04; H, 6.47; N, 12.84. Found: C, 66.10; H, 6.33; N, 12.76.

$\mathbf{N}$-(Cyclohex-2-enyl)-4-nitrobenzenamine (Table 3, Entry 2) The general procedure was followed with the exception that the reaction was run at $50^{\circ} \mathrm{C}$ with 4-nitrobenzenamine $(138 \mathrm{mg}$, $1.00 \mathrm{mmol}$ ). The reaction mixture was purified by flash column chromatography to give $217 \mathrm{mg}$ (99\%) of the hydroamination product: ${ }^{1} \mathrm{H} \mathrm{NMR}\left(500 \mathrm{MHz}, \mathrm{CDCl}_{3}, \mathrm{TMS}\right) \delta 1.58-1.73$ (m, 3H), 
$1.90(\mathrm{~m}, 1 \mathrm{H}), 2.00(\mathrm{~m}, 2 \mathrm{H}), 4.03(\mathrm{~s}, 1 \mathrm{H}), 4.82(\mathrm{~d}, J=8.0 \mathrm{~Hz}, 1 \mathrm{H}), 5.64(\mathrm{~m}, 1 \mathrm{H}), 5.87(\mathrm{~m}, 1 \mathrm{H})$, $6.51(\mathrm{ddd}, J=9.5,3.0,2.0 \mathrm{~Hz}, 2 \mathrm{H}), 8.01$ (ddd, $J=9.0,3.0,2.0 \mathrm{~Hz}, 2 \mathrm{H}) ;{ }^{13} \mathrm{C}\left\{{ }^{1} \mathrm{H}\right\} \mathrm{NMR}(126$ $\left.\mathrm{MHz}, \mathrm{CDCl}_{3}\right) \delta 19.2,24.7,28.5,47.5,111.1,126.3,126.3,131.3,137.1,152.4$; Anal. Calcd for $\mathrm{C}_{12} \mathrm{H}_{14} \mathrm{~N}_{2} \mathrm{O}_{2}$ : C, 66.04; H, 6.47; N, 12.84. Found: C, 66.13; H, 6.58; N, 12.94.

3-(Cyclohex-2-enylamino)benzoic acid (Table 3, Entry 3) The general procedure was followed with 3 -aminobenzoic acid $(137 \mathrm{mg}, 1.00 \mathrm{mmol})$. The reaction mixture was purified by flash column chromatography to give $176 \mathrm{mg}(81 \%)$ of the hydroamination product: ${ }^{1} \mathrm{H}$ NMR $\left(500 \mathrm{MHz}, \mathrm{CDCl}_{3}, \mathrm{TMS}\right) \delta$ 1.60-1.75 (m, 3H), $1.93(\mathrm{~m}, 1 \mathrm{H}), 2.04(\mathrm{~m}, 2 \mathrm{H}), 4.05(\mathrm{~s}, 1 \mathrm{H}), 4.82(\mathrm{~d}$, $J=8.0 \mathrm{~Hz}, 1 \mathrm{H}), 5.74(\mathrm{~m}, 1 \mathrm{H}), 5.87(\mathrm{~m}, 1 \mathrm{H}), 6.84(\mathrm{dd}, J=8.5,3.0, \mathrm{~Hz}, 1 \mathrm{H}), 7.24$ (t, $J=7.5 \mathrm{~Hz}$, $1 \mathrm{H}), 7.35(\mathrm{t}, J=1.5 \mathrm{~Hz}, 1 \mathrm{H}), 7.43(\mathrm{dt}, J=7.5,1.5 \mathrm{~Hz}, 1 \mathrm{H}), 7.86($ br s, $1 \mathrm{H}) ;{ }^{13} \mathrm{C}\left\{{ }^{1} \mathrm{H}\right\} \mathrm{NMR}(126$ $\left.\mathrm{MHz}, \mathrm{CDCl}_{3}\right) \delta 19.6,25.1,28.7,47.9,114.2,118.6,118.9,128.0,129.3,130.3,130.5,147.2$, 172.7; Anal. Calcd for $\mathrm{C}_{13} \mathrm{H}_{15} \mathrm{NO}_{2}$ : C, 71.87; H, 6.96; N, 6.45. Found: C, 71.60; H, 6.92; N, 6.21. 1-(3-(Cyclohex-2-enylamino)phenyl)ethanone (Table 3, Entry 4) The general procedure was followed with 1-(3-aminophenyl)ethanone $(135 \mathrm{mg}, 1.00 \mathrm{mmol})$. The reaction mixture was purified by flash column chromatography to give $199 \mathrm{mg}(93 \%)$ of the hydroamination product: ${ }^{1} \mathrm{H}$ NMR (500 MHz, $\mathrm{CDCl}_{3}$, TMS) $\delta$ 1.55-1.72 (m, 3H), $1.88(\mathrm{~m}, 1 \mathrm{H}), 2.00(\mathrm{~m}, 2 \mathrm{H}), 2.52(\mathrm{~s}$, $3 \mathrm{H}), 3.92$ (br s, 1H), $4.00(\mathrm{~s}, 1 \mathrm{H}), 5.69(\mathrm{~m}, 1 \mathrm{H}), 5.82(\mathrm{~m}, 1 \mathrm{H}), 6.84(\mathrm{dt}, J=6.5,2.5 \mathrm{~Hz}, 1 \mathrm{H})$, 7.18-7.22 (m, 3H); ${ }^{13} \mathrm{C}\left\{{ }^{1} \mathrm{H}\right\}$ NMR $\left(126 \mathrm{MHz}, \mathrm{CDCl}_{3}\right) \delta$ 19.4, 24.8, 26.4, 28.5, 47.6, 111.7, 117.1, 117.6, 127.9, 129.1, 130.1, 137.9, 147.2, 198.4; Anal. Calcd for $\mathrm{C}_{14} \mathrm{H}_{17} \mathrm{NO}$ : C, 78.10; $\mathrm{H}$, 7.96; N, 6.51. Found: C, 77.87; H, 7.99; N, 6.68.

1-(4-(Cyclohex-2-enylamino)phenyl)ethanone (Table 3, Entry 5) The general procedure was followed with 1-(4-aminophenyl)ethanone $(135 \mathrm{mg}, 1.00 \mathrm{mmol})$. The reaction mixture was purified by flash column chromatography to give $214 \mathrm{mg}(99 \%)$ of the hydroamination product: ${ }^{1} \mathrm{H}$ NMR (500 MHz, $\mathrm{CDCl}_{3}$, TMS) $\delta$ 1.50-1.66 (m, 3H), $1.83(\mathrm{~m}, 1 \mathrm{H}), 1.94(\mathrm{~m}, 2 \mathrm{H}), 2.40$ (s, 3H), 3.96 (br s, 1H), 4.69 (d, $J=8.0 \mathrm{~Hz}, 1 \mathrm{H}), 5.62$ (m, 1H), 5.78 (m, 1H), 6.51 (d, J=9.0 Hz, 2H), $7.74(\mathrm{~d}, J=9.0 \mathrm{~Hz}, 2 \mathrm{H}) ;{ }^{13} \mathrm{C}\left\{{ }^{1} \mathrm{H}\right\} \mathrm{NMR}\left(126 \mathrm{MHz}, \mathrm{CDCl}_{3}\right) \delta 19.1,24.6,25.4,28.2,47.0$, 111.2, 125.6, 127.1, 130.4, 151.1, 195.7; Anal. Calcd for $\mathrm{C}_{14} \mathrm{H}_{17} \mathrm{NO}$ : C, 78.10; H, 7.96; N, 6.51. Found: C, 77.93; H, 8.24; N, 6.55.

N-(Cyclohex-2-enyl)-2-(methylthio)benzenamine (Table 3, Entry 6) The general procedure was followed with 2-(methylthio)benzenamine $(139 \mathrm{mg}, 1.00 \mathrm{mmol})$. The reaction mixture was purified by flash column chromatography to give $215 \mathrm{mg}(98 \%)$ of the hydroamination product: 
${ }^{1} \mathrm{H}$ NMR (500 MHz, $\mathrm{CDCl}_{3}$, TMS) $\delta ~ 1.58-1.75(\mathrm{~m}, 3 \mathrm{H}), 1.88(\mathrm{~m}, 1 \mathrm{H}), 2.02(\mathrm{~m}, 2 \mathrm{H}), 2.27$ (s, $3 \mathrm{H}), 4.01(\mathrm{~s}, 1 \mathrm{H}), 4.94(\mathrm{~s}, 1 \mathrm{H}), 5.75(\mathrm{~m}, 1 \mathrm{H}), 5.85(\mathrm{~m}, 1 \mathrm{H}), 6.60(\mathrm{t}, J=7.5 \mathrm{~Hz}, 1 \mathrm{H}), 6.64(\mathrm{~d}, J=$ $8.5 \mathrm{~Hz}, 1 \mathrm{H}), 7.14(\mathrm{t}, J=8.5 \mathrm{~Hz}, 1 \mathrm{H}), 7.38(\mathrm{~d}, J=7.5 \mathrm{~Hz}, 1 \mathrm{H}) ;{ }^{13} \mathrm{C}\left\{{ }^{1} \mathrm{H}\right\} \mathrm{NMR}\left(126 \mathrm{MHz}, \mathrm{CDCl}_{3}\right)$ $\delta 18.0,19.5,25.1,28.6,47.5,110.4,116.5,119.6,128.2$, 129.3, 130.2, 134.3, 147.1; Anal. Calcd for $\mathrm{C}_{13} \mathrm{H}_{17} \mathrm{NS}$ : C, 71.18; H, 7.81; N, 6.39. Found: C, 71.45; H, 7.65; N, 6.60.

$N$-(Cyclohex-2-enyl)-4-(methylthio)benzenamine (Table 3, Entry 7) The general procedure was followed with 4-(methylthio)benzenamine $(139 \mathrm{mg}, 1.00 \mathrm{mmol})$. The reaction mixture was purified by flash column chromatography to give $217 \mathrm{mg}(99 \%)$ of the hydroamination product: ${ }^{1} \mathrm{H}$ NMR (500 MHz, $\mathrm{CDCl}_{3}$, TMS) $\delta$ 1.57-1.68 (m, 3H), $1.86(\mathrm{~m}, 1 \mathrm{H}), 2.00(\mathrm{~m}, 2 \mathrm{H}), 2.37$ (s, $3 \mathrm{H}), 3.68(\mathrm{~s}, 1 \mathrm{H}), 3.93(\mathrm{~s}, 1 \mathrm{H}), 5.70(\mathrm{~m}, 1 \mathrm{H}), 5.83(\mathrm{~m}, 1 \mathrm{H}), 6.53(\mathrm{~d}, J=9.0 \mathrm{~Hz}, 2 \mathrm{H}), 7.18(\mathrm{~d}, J=$ $9.0 \mathrm{~Hz}, 2 \mathrm{H}) ;{ }^{13} \mathrm{C}\left\{{ }^{1} \mathrm{H}\right\}$ NMR $\left(126 \mathrm{MHz}, \mathrm{CDCl}_{3}\right) \delta 19.0,19.4,24.9,28.6,47.7,113.5,123.5$, 128.1, 130.0, 131.4, 145.9; Anal. Calcd for $\mathrm{C}_{13} \mathrm{H}_{17} \mathrm{NS}$ : C, 71.18; H, 7.81; N, 6.39. Found: C, 71.46; H, 7.77; N, 6.53 .

2-(Cyclohex-2-enylamino)benzonitrile (Table 3, Entry 8) The general procedure was followed with the following exceptions: the reaction was run at $50{ }^{\circ} \mathrm{C}$ in $1.0 \mathrm{~mL}$ of THF with $1,3-$ cyclohexadiene $(382 \mu \mathrm{L}, 4.00 \mathrm{mmol})$ and 2-aminobenzonitrile $(118 \mathrm{mg}, 1.00 \mathrm{mmol})$. The reaction mixture was purified by flash column chromatography to give $194 \mathrm{mg}(98 \%)$ of the hydroamination product: ${ }^{1} \mathrm{H}$ NMR $\left(500 \mathrm{MHz}, \mathrm{CDCl}_{3}\right.$, TMS) $\delta 1.60-1.77(\mathrm{~m}, 3 \mathrm{H}), 1.91(\mathrm{~m}, 1 \mathrm{H})$, $2.06(\mathrm{~m}, 2 \mathrm{H}), 4.06(\mathrm{~d}, J=2.0 \mathrm{~Hz}, 1 \mathrm{H}), 4.54(\mathrm{~d}, J=7.5 \mathrm{~Hz}, 1 \mathrm{H}), 5.70(\mathrm{~m}, 1 \mathrm{H}), 5.91(\mathrm{~m}, 1 \mathrm{H}), 6.64$ $(\mathrm{t}, J=7.5 \mathrm{~Hz}, 1 \mathrm{H}), 6.71(\mathrm{~d}, J=8.5 \mathrm{~Hz}, 1 \mathrm{H}), 7.37(\mathrm{~d}, J=7.5 \mathrm{~Hz}, 2 \mathrm{H}) ;{ }^{13} \mathrm{C}\left\{{ }^{1} \mathrm{H}\right\} \mathrm{NMR}(126 \mathrm{MHz}$, $\left.\mathrm{CDCl}_{3}\right) \delta 19.3,24.8,28.4,47.5,95.8,110.9,116.1,117.8,126.8,131.3,132.8,134.1,149.2$; Anal. Calcd for $\mathrm{C}_{13} \mathrm{H}_{14} \mathrm{~N}_{2}$ : C, 78.75; H, 7.12; N, 14.13. Found: C, 78.91; H, 6.92; N, 13.85.

3-(Cyclohex-2-enylamino)benzonitrile (Table 3, Entry 9) The general procedure was followed with 3-aminobenzonitrile $(118.1 \mathrm{mg}, 1.00 \mathrm{mmol})$. The reaction mixture was purified by flash column chromatography to give $196 \mathrm{mg}(99 \%)$ of the hydroamination product: ${ }^{1} \mathrm{H}$ NMR (500 $\mathrm{MHz}, \mathrm{CDCl}_{3}$, TMS) $\delta$ 1.56-1.72 (m, 3H), $1.87(\mathrm{~m}, 1 \mathrm{H}), 2.02(\mathrm{~m}, 2 \mathrm{H}), 3.92(\mathrm{~s}, 1 \mathrm{H}), 4.03(\mathrm{~s}, 1 \mathrm{H})$, $5.67(\mathrm{~m}, 1 \mathrm{H}), 5.86(\mathrm{~m}, 1 \mathrm{H}), 6.77(\mathrm{~m}, 2 \mathrm{H}), 6.87(\mathrm{dt}, J=7.5,1.5 \mathrm{~Hz}, 1 \mathrm{H}), 7.17$ (ddd, $J=7.5,7.5$, $2.0 \mathrm{~Hz}, 1 \mathrm{H}) ;{ }^{13} \mathrm{C}\left\{{ }^{1} \mathrm{H}\right\} \mathrm{NMR}\left(126 \mathrm{MHz}, \mathrm{CDCl}_{3}\right) \delta 19.2,24.8,28.2,47.4,112.6,114.8,117.3$, 119.3, 119.9, 127.2, 129.7, 130.3, 147.2; Anal. Calcd for $\mathrm{C}_{13} \mathrm{H}_{14} \mathrm{~N}_{2}$ : C, 78.75; H, 7.12; N, 14.13. Found: C, 78.53; H, 6.97; N, 13.97. 
4-(Cyclohex-2-enylamino)benzonitrile (Table 3, Entry 10) The general procedure was followed with 4-aminobenzonitrile $(118 \mathrm{mg}, 1.00 \mathrm{mmol})$. The reaction mixture was purified by flash column chromatography to give $192 \mathrm{mg}$ (97\%) of the hydroamination product: ${ }^{1} \mathrm{H}$ NMR (500 MHz, $\left.\mathrm{CDCl}_{3}, \mathrm{TMS}\right) \delta$ 1.60-1.75 (m, 3H), 1.89 (m, 1H), 2.04 (m, 2H), 4.00 (d, J=2.5 Hz, $1 \mathrm{H}), 4.42(\mathrm{~d}, J=7.5 \mathrm{~Hz}, 1 \mathrm{H}), 5.68(\mathrm{~m}, 1 \mathrm{H}), 5.90(\mathrm{~m}, 1 \mathrm{H}), 6.56(\mathrm{~d}, J=9.0 \mathrm{~Hz}, 2 \mathrm{H}), 7.37(\mathrm{~d}, J=$ $9.0 \mathrm{~Hz}, 2 \mathrm{H}) ;{ }^{13} \mathrm{C}\left\{{ }^{1} \mathrm{H}\right\}$ NMR $\left(126 \mathrm{MHz}, \mathrm{CDCl}_{3}\right) \delta 19.2,24.7,28.2,47.2,97.7,112.2,120.4$, 126.8, 131.0, 133.4, 150.2; Anal. Calcd for $\mathrm{C}_{13} \mathrm{H}_{14} \mathrm{~N}_{2}$ : C, 78.75; H, 7.12; N, 14.13. Found: C, 78.49; H, 7.40; N, 13.85 .

Ethyl 2-(cyclohex-2-enylamino)benzoate (Table 3, Entry 11) The general procedure was followed with ethyl 2-aminobenzoate $(165 \mathrm{mg}, 1.00 \mathrm{mmol})$. The reaction mixture was purified by flash column chromatography to give $215 \mathrm{mg}(88 \%)$ of the hydroamination product: ${ }^{1} \mathrm{H}$ NMR (400 MHz, $\left.\mathrm{CDCl}_{3}, \mathrm{TMS}\right) \delta 1.34(\mathrm{t}, J=7.2 \mathrm{~Hz}, 3 \mathrm{H}), 1.58-1.79(\mathrm{~m}, 3 \mathrm{H}), 1.89(\mathrm{~m}, 1 \mathrm{H}), 2.03(\mathrm{~m}$, $2 \mathrm{H}), 4.05(\mathrm{~d}, J=2.0 \mathrm{~Hz}, 1 \mathrm{H}), 4.28(\mathrm{q}, J=7.2 \mathrm{~Hz}, 2 \mathrm{H}), 5.74(\mathrm{~m}, 1 \mathrm{H}), 5.85(\mathrm{~m}, 1 \mathrm{H}), 6.53$ (ddd, $J$ $=7.8,7.8,1.2 \mathrm{~Hz}, 1 \mathrm{H}), 6.70(\mathrm{~d}, J=8.4 \mathrm{~Hz}, 1 \mathrm{H}), 7.29(\mathrm{ddd}, J=7.8,7.8,1.2 \mathrm{~Hz}, 1 \mathrm{H}), 7.82(\mathrm{~d}, J=$ $7.6 \mathrm{~Hz}, 1 \mathrm{H}), 7.92(\mathrm{dd}, J=8.2,1.8 \mathrm{~Hz}, 1 \mathrm{H}) ;{ }^{13} \mathrm{C}\left\{{ }^{1} \mathrm{H}\right\} \mathrm{NMR}\left(101 \mathrm{MHz}, \mathrm{CDCl}_{3}\right) \delta$ 14.2, 19.5, 24.9, 28.6, 46.6, 59.9, 110.0, 111.3, 114.0, 127.7, 130.1, 131.7, 134.2, 150.0, 168.4; Anal. Calcd for $\mathrm{C}_{15} \mathrm{H}_{19} \mathrm{NO}_{2}: \mathrm{C}, 73.44 ; \mathrm{H}, 7.81 ; \mathrm{N}, 5.71$. Found: C, 73.23; H, 7.55; N, 5.88.

Ethyl 3-(cyclohex-2-enylamino)benzoate (Table 3, Entry 12) The general procedure was followed with ethyl 3-aminobenzoate $(165 \mathrm{mg}, 1.00 \mathrm{mmol})$. The reaction mixture was purified by flash column chromatography to give $201 \mathrm{mg}(82 \%)$ of the hydroamination product: ${ }^{1} \mathrm{H} \mathrm{NMR}$ (400 MHz, $\mathrm{CDCl}_{3}$, TMS) $\delta 1.35(\mathrm{t}, J=7.2 \mathrm{~Hz}, 3 \mathrm{H}), 1.53-1.72(\mathrm{~m}, 3 \mathrm{H}), 1.87(\mathrm{~m}, 1 \mathrm{H}), 1.99$ (m, 2H), $3.92(\mathrm{~s}, 1 \mathrm{H}), 4.00(\mathrm{~s}, 1 \mathrm{H}), 4.33$ (q, $J=7.2 \mathrm{~Hz}, 2 \mathrm{H}), 5.70(\mathrm{~m}, 1 \mathrm{H}), 5.82(\mathrm{~m}, 1 \mathrm{H}), 6.76$ (ddd, $J$ $=8.0,1.6,0.4 \mathrm{~Hz}, 1 \mathrm{H}), 7.20(\mathrm{t}, J=8.0 \mathrm{~Hz}, 1 \mathrm{H}), 7.36(\mathrm{~m}, 2 \mathrm{H}) ;{ }^{13} \mathrm{C}\left\{{ }^{1} \mathrm{H}\right\} \mathrm{NMR}\left(101 \mathrm{MHz}, \mathrm{CDCl}_{3}\right)$ $\delta$ 14.0, 19.3, 24.8, 28.4, 47.5, 60.5, 113.5, 117.1, 117.8, 127.9, 128.8, 130.0, 131.0, 147.0, 166.7; Anal. Calcd for $\mathrm{C}_{15} \mathrm{H}_{19} \mathrm{NO}_{2}$ : C, 73.44; H, 7.81; N, 5.71. Found: C, 73.19; H, 7.94; N, 5.56.

Ethyl 4-(cyclohex-2-enylamino)benzoate (Table 3, Entry 13) The general procedure was followed with ethyl 4-aminobenzoate $(165 \mathrm{mg}, 1.00 \mathrm{mmol})$. The reaction mixture was purified by flash column chromatography to give $234 \mathrm{mg}(95 \%)$ of the hydroamination product: ${ }^{1} \mathrm{H}$ NMR $\left(500 \mathrm{MHz}, \mathrm{CDCl}_{3}, \mathrm{TMS}\right) \delta 1.33(\mathrm{t}, J=7.0 \mathrm{~Hz}, 3 \mathrm{H}), 1.53-1.71(\mathrm{~m}, 3 \mathrm{H}), 1.88(\mathrm{~m}, 1 \mathrm{H}), 1.99(\mathrm{~m}$, $2 \mathrm{H}), 4.00(\mathrm{~s}, 1 \mathrm{H}), 4.29(\mathrm{q}, J=7.0 \mathrm{~Hz}, 2 \mathrm{H}), 4.41(\mathrm{~d}, J=7.5,1 \mathrm{H}), 5.66(\mathrm{~m}, 1 \mathrm{H}), 5.83(\mathrm{~m}, 1 \mathrm{H})$, $6.54(\mathrm{~d}, J=9.0 \mathrm{~Hz}, 2 \mathrm{H}), 7.85(\mathrm{t}, J=9.0 \mathrm{~Hz}, 2 \mathrm{H}), 7.36(\mathrm{~m}, 2 \mathrm{H}) ;{ }^{13} \mathrm{C}\left\{{ }^{1} \mathrm{H}\right\} \mathrm{NMR}(126 \mathrm{MHz}$, 
$\left.\mathrm{CDCl}_{3}\right) \delta 14.1,19.2,24.7,28.3,47.1,59.7,111.3,117.7,127.3,130.3,131.2,150.8,166.5$; Anal. Calcd for $\mathrm{C}_{15} \mathrm{H}_{19} \mathrm{NO}_{2}$ : C, 73.44; $\mathrm{H}, 7.81 ; \mathrm{N}$, 5.71. Found: C, 73.35; H, 8.11; N, 5.55.

2-(Cyclohex-2-enylamino)phenol (Table 3, Entry 14) The general procedure was followed with the following exceptions: the reaction was run at $50{ }^{\circ} \mathrm{C}$ in $1.0 \mathrm{~mL}$ of THF with $1,3-$ cyclohexadiene $(382 \mu \mathrm{L}, 4.00 \mathrm{mmol})$ and 2-aminophenol $(109 \mathrm{mg}, 1.00 \mathrm{mmol})$. The reaction mixture was purified by flash column chromatography to give $109 \mathrm{mg}(57 \%)$ of the hydroamination product: ${ }^{1} \mathrm{H}$ NMR (500 MHz, $\mathrm{CDCl}_{3}$, TMS) $\delta 1.63(\mathrm{~m}, 2 \mathrm{H}), 1.77(\mathrm{~m}, 1 \mathrm{H}), 1.95$ (m, 1H), 2.06 (m, 2H), 4.00 (br s, 1H), 5.09 (br s, 2H), 5.79-5.90 (m, 2H), 6.66 (br s, 2H), 6.79$6.90(\mathrm{~m}, 2 \mathrm{H}) ;{ }^{13} \mathrm{C}\left\{{ }^{1} \mathrm{H}\right\}$ NMR $\left(126 \mathrm{MHz}, \mathrm{CDCl}_{3}\right) \delta$ 19.6, 25.0, 28.7, 48.7, 113.8, 114.7, 118.2, 121.2, 128.4, 130.0, 135.5, 144.4; Anal. Calcd for $\mathrm{C}_{15} \mathrm{H}_{19} \mathrm{NO}_{2}: \mathrm{C}, 76.16 ; \mathrm{H}, 8.12 ; \mathrm{N}, 7.40$. Found: C, 76.14; H, 8.12; N, 7.24.

General Procedure for the Hydroamination of Styrene with Functionalized Anilines (Table 4). In a drybox, XantphosPd( $\eta^{3}$-allyl)OTf (2) $\left(17.5 \mathrm{mg}, 2.00 \times 10^{-2} \mathrm{mmol}\right)$, styrene (229 $\mu \mathrm{L}$, $2.00 \mathrm{mmol})$, and the aniline $(1.00 \mathrm{mmol})$ were placed into a small vial, dissolved in $1.00 \mathrm{~mL}$ of 1,4-dioxane and sealed with a cap containing a PTFE septum. Trifluoromethanesulfonic acid ( $8.8 \mu \mathrm{L}, 1.00 \times 10^{-1} \mathrm{mmol}$ ) was subsequently added by syringe to the reaction. The resulting reaction mixture was stirred at $100{ }^{\circ} \mathrm{C}$ for $12 \mathrm{~h}$. Upon completion, the reaction mixture was directly adsorbed onto silica gel and purified by flash column chromatography.

2-(1-Phenylethylamino)phenol (Table 4, Entry 1) The general procedure was followed with ethyl 2-aminophenol $(109 \mathrm{mg}, 1.00 \mathrm{mmol})$. The reaction mixture was purified by flash column chromatography to give $151 \mathrm{mg}(71 \%)$ of the hydroamination product: ${ }^{1} \mathrm{H}$ NMR (400 MHz, $\left.\mathrm{CDCl}_{3}, \mathrm{TMS}\right) \delta 1.50(\mathrm{~d}, J=6.8 \mathrm{~Hz}, 3 \mathrm{H}), 4.45(\mathrm{~d}, J=5.2 \mathrm{~Hz}, 1 \mathrm{H}), 4.87$ (br s, 2H), 6.45 (d, $J=$ $7.6 \mathrm{~Hz}, 1 \mathrm{H}), 6.59(\mathrm{~m}, 3 \mathrm{H}), 7.17(\mathrm{~m}, 1 \mathrm{H}), 7.27(\mathrm{t}, J=7.6,2 \mathrm{H}), 7.33(\mathrm{~d}, J=7.2,1 \mathrm{H}) ;{ }^{13} \mathrm{C}\left\{{ }^{1} \mathrm{H}\right\}$ NMR (101 MHz, $\left.\mathrm{CDCl}_{3}\right) \delta 24.8,53.8,113.7,114.3,117.7,121.4,125.8,126.8,128.5,135.8$, 143.4, 145.0; Anal. Calcd for $\mathrm{C}_{14} \mathrm{H}_{15} \mathrm{NO}$ : C, 78.84; H, 7.09; N, 6.57. Found: C, 78.83; H, 6.91; $\mathrm{N}, 6.51$.

2-(4-(1-Phenylethylamino)phenyl)ethanol (Table 4, Entry 2) The general procedure was followed with 2-(4-aminophenyl)ethanol $(137 \mathrm{mg}, 1.00 \mathrm{mmol})$. The reaction mixture was purified by flash column chromatography to give $189 \mathrm{mg}$ (79\%) of the hydroamination product: ${ }^{1} \mathrm{H}$ NMR (400 MHz, CDCl 3 , TMS) $\delta 1.43$ (d, $J=6.8 \mathrm{~Hz}, 3 \mathrm{H}$ ), 2.20 (br s, $1 \mathrm{H}$ ), 2.60 (t, $J=6.4 \mathrm{~Hz}$, 2H), 3.61 (t, $J=6.4 \mathrm{~Hz}, 2 \mathrm{H}), 3.90$ (br s, $1 \mathrm{H}), 4.39$ (q, $J=6.6 \mathrm{~Hz}, 1 \mathrm{H}), 6.41$ (d, $J=8.0 \mathrm{~Hz}, 2 \mathrm{H}$ ), 
$6.87(\mathrm{~d}, J=8.0 \mathrm{~Hz}, 2 \mathrm{H}), 7.16(\mathrm{t}, J=7.2 \mathrm{~Hz}, 1 \mathrm{H}), 7.25(\mathrm{t}, J=7.6,2 \mathrm{H}), 7.31(\mathrm{~d}, J=7.2,2 \mathrm{H})$; ${ }^{13} \mathrm{C}\left\{{ }^{1} \mathrm{H}\right\}$ NMR $\left(101 \mathrm{MHz}, \mathrm{CDCl}_{3}\right) \delta 24.7,38.0,53.3,63.5,113.2,125.6,126.6,126.7,128.4$, 129.4, 145.1, 145.6; Anal. Calcd for $\mathrm{C}_{16} \mathrm{H}_{19} \mathrm{NO}$ : C, 79.63; H, 7.94; N, 5.80. Found: C, 79.40; H, $8.09 ; \mathrm{N}, 5.72$.

3-(1-Phenylethylamino)benzoic acid (Table 4, Entry 3) The general procedure was followed with 3-aminobenzoic acid $(137 \mathrm{mg}, 1.00 \mathrm{mmol})$. The reaction mixture was purified by flash column chromatography to give $184 \mathrm{mg}(76 \%)$ of the hydroamination product: ${ }^{1} \mathrm{H}$ NMR (500 MHz, $\mathrm{CDCl}_{3}$, TMS) $\delta 1.58(\mathrm{~d}, J=6.5 \mathrm{~Hz}, 3 \mathrm{H}), 4.59(\mathrm{q}, J=6.5 \mathrm{~Hz}, 1 \mathrm{H}), 6.74(\mathrm{dd}, J=8.5,2.5$ $\mathrm{Hz}, 1 \mathrm{H}), 7.20(\mathrm{t}, J=8.5 \mathrm{~Hz}, 2 \mathrm{H}), 7.28(\mathrm{~m}, 1 \mathrm{H}), 7.35-7.45(\mathrm{~m}, 6 \mathrm{H}), 7.90($ br s, $2 \mathrm{H}) ;{ }^{13} \mathrm{C}\left\{{ }^{1} \mathrm{H}\right\}$ NMR $\left(126 \mathrm{MHz}, \mathrm{CDCl}_{3}\right) \delta 24.7,53.3,114.7,118.2,119.0,125.8,127.0,128.7,129.1,130.0$, 144.5, 147.2, 172.6; Anal. Calcd for $\mathrm{C}_{15} \mathrm{H}_{15} \mathrm{NO}_{2}$ : C, 74.67; H, 6.27; N, 5.81. Found: C, 74.39; H, $6.11 ; \mathrm{N}, 5.70$.

1-(3-(1-Phenylethylamino)phenyl)ethanone (Table 4, Entry 4) The general procedure was followed with 1-(3-aminophenyl)ethanone $(135 \mathrm{mg}, 1.00 \mathrm{mmol})$ with the following exception; $\left[\mathrm{Pd}\left(\mathrm{CH}_{3} \mathrm{CN}\right)_{4}\right]\left(\mathrm{BF}_{4}\right)_{2}\left(8.9 \mathrm{mg}, 2.00 \times 10^{-2} \mathrm{mmol}\right)$ and Xantphos $\left(11.6 \mathrm{mg}, 2.00 \times 10^{-2} \mathrm{mmol}\right)$ were used in place of $\operatorname{XantphosPd}\left(\eta^{3}\right.$-allyl)OTf. The reaction mixture was purified by flash column chromatography to give $137 \mathrm{mg}(57 \%)$ of the hydroamination product: ${ }^{1} \mathrm{H}$ NMR (400 MHz, $\mathrm{CDCl}_{3}$, TMS) $\delta 1.50$ (d, $\left.J=6.8 \mathrm{~Hz}, 3 \mathrm{H}\right), 2.45$ (s, $\left.3 \mathrm{H}\right), 4.33$ (d, $\left.J=4.8 \mathrm{~Hz}, 1 \mathrm{H}\right), 4.51$ (dq, $\left.J_{\mathrm{NH}}=4.8 \mathrm{~Hz}, J_{\mathrm{CH}}=6.8 \mathrm{~Hz}, 1 \mathrm{H}\right), 6.65(\mathrm{dd}, J=7.6,2.4 \mathrm{~Hz}, 1 \mathrm{H}), 7.12(\mathrm{~m}, 2 \mathrm{H}), 7.20(\mathrm{~m}, 2 \mathrm{H})$, 7.23-7.36 (m, 4H); ${ }^{13} \mathrm{C}\left\{{ }^{1} \mathrm{H}\right\}$ NMR $\left(101 \mathrm{MHz}, \mathrm{CDCl}_{3}\right) \delta$ 24.7, 26.5, 53.2, 112.5, 117.2, 117.5, 125.7, 126.9, 128.6, 129.1, 137.7, 144.5, 147.3, 198.5; Anal. Calcd for $\mathrm{C}_{16} \mathrm{H}_{17} \mathrm{NO}$ : C, 80.30; $\mathrm{H}$, 7.16; N, 5.85. Found: C, 80.17; H, 7.02; N, 5.82.

1-(4-(1-Phenylethylamino)phenyl)ethanone (Table 4, Entry 5) The general procedure was followed with 1-(4-aminophenyl)ethanone $(135 \mathrm{mg}, 1.00 \mathrm{mmol})$. The reaction mixture was purified by flash column chromatography to give $112 \mathrm{mg}$ (47\%) of the hydroamination product: ${ }^{1} \mathrm{H}$ NMR (400 MHz, $\mathrm{CDCl}_{3}$, TMS) $\delta 1.52$ (d, $\left.J=6.8 \mathrm{~Hz}, 3 \mathrm{H}\right), 2.42(\mathrm{~s}, 3 \mathrm{H}), 4.55$ (dq, $J_{\mathrm{NH}}=4.8$ $\left.\mathrm{Hz} J_{\mathrm{CH}}=6.8 \mathrm{~Hz}, 1 \mathrm{H}\right), 4.83(\mathrm{~d}, J=4.8 \mathrm{~Hz}, 1 \mathrm{H}), 6.47(\mathrm{~d}, J=8.8 \mathrm{~Hz}, 2 \mathrm{H}), 7.22(\mathrm{~m}, 1 \mathrm{H}), 7.30(\mathrm{~m}$, 4H), $7.72(\mathrm{~d}, J=8.8 \mathrm{~Hz}, 2 \mathrm{H}) ;{ }^{13} \mathrm{C}\left\{{ }^{1} \mathrm{H}\right\} \mathrm{NMR}\left(101 \mathrm{MHz}, \mathrm{CDCl}_{3}\right) \delta 24.5,25.9,52.9,111.9,125.6$, 126.4, 127.0, 128.6, 130.5, 144.0, 151.1, 196.3; Anal. Calcd for $\mathrm{C}_{16} \mathrm{H}_{17} \mathrm{NO}$ : C, 80.30; H, 7.16; N, 5.85. Found: $\mathrm{C}, 80.03 ; \mathrm{H}, 7.08 ; \mathrm{N}, 5.82$. 
2-(Methylthio)-N-(1-phenylethyl)benzenamine (Table 4, Entry 6) The general procedure was followed with 2-(methylthio)benzenamine $(139 \mathrm{mg}, 1.00 \mathrm{mmol})$. The reaction mixture was purified by flash column chromatography to give $133 \mathrm{mg}(57 \%)$ of the hydroamination product: ${ }^{1} \mathrm{H}$ NMR (500 MHz, $\mathrm{CDCl}_{3}$, TMS) $\delta 1.53$ (d, $J=6.5 \mathrm{~Hz}, 3 \mathrm{H}$ ), 2.32 (s, 3H), 4.47 (q, $J=6.0 \mathrm{~Hz}$, 1H), 5.33 (br s, 1H), 6.34 (d, $J=8.5 \mathrm{~Hz}, 1 \mathrm{H}), 6.55$ (dt, $J=7.8,1.0 \mathrm{~Hz}, 1 \mathrm{H}), 6.96$ (dt, $J=8.5,1.5$ $\mathrm{Hz}, 1 \mathrm{H}), 7.17$ (t, $J=7.5 \mathrm{~Hz}, 1 \mathrm{H}), 7.26$ (t, $J=8.0 \mathrm{~Hz}, 2 \mathrm{H}), 7.31$ (d, $J=7.0 \mathrm{~Hz}, 2 \mathrm{H}), 7.36$ (dd, $J=$ 7.5, $1.0 \mathrm{~Hz}, 1 \mathrm{H}) ;{ }^{13} \mathrm{C}\left\{{ }^{1} \mathrm{H}\right\} \mathrm{NMR}\left(126 \mathrm{MHz}, \mathrm{CDCl}_{3}\right) \delta 18.1,25.1,53.3,111.3,116.9,119.4$, 125.6, 126.8, 128.5, 129.2, 133.8, 144.9, 147.1; Anal. Calcd for $\mathrm{C}_{15} \mathrm{H}_{17} \mathrm{NS}$ : C, 74.03; H, 7.04; N, 5.76. Found: C, 74.32; H, 6.87; N, 5.51.

4-(Methylthio)-N-(1-phenylethyl)benzenamine (Table 4, Entry 7) The general procedure excluding the trifluoromethanesulfonic acid cocatalyst was followed with 4(methylthio)benzenamine $(139 \mathrm{mg}, 1.00 \mathrm{mmol})$. The reaction mixture was purified by flash column chromatography to give $215 \mathrm{mg}(88 \%)$ of the hydroamination product: ${ }^{1} \mathrm{H}$ NMR (500 $\mathrm{MHz}, \mathrm{CDCl}_{3}$, TMS) $\delta 1.44$ (d, $\left.J=7.0 \mathrm{~Hz}, 3 \mathrm{H}\right), 2.31$ (s, 3H), 4.04 (br s, 1H), 4.41 (q, $J=6.5 \mathrm{~Hz}$, $1 \mathrm{H}), 6.41(\mathrm{~d}, J=8.5 \mathrm{~Hz}, 2 \mathrm{H}), 7.09$ (d, $J=8 \mathrm{~Hz}, 2 \mathrm{H}), 7.18(\mathrm{t}, J=7 \mathrm{~Hz}, 1 \mathrm{H}), 7.25-7.31(\mathrm{~m}, 4 \mathrm{H})$; ${ }^{13} \mathrm{C}\left\{{ }^{1} \mathrm{H}\right\}$ NMR $\left(126 \mathrm{MHz}, \mathrm{CDCl}_{3}\right) \delta 18.8,24.8,53.3,113.8,123.8,125.6,126.8,128.5,131.1$, 144.8, 145.9; Anal. Calcd for $\mathrm{C}_{15} \mathrm{H}_{17} \mathrm{NS}$ : C, 74.03; H, 7.04; N, 5.76. Found: C, 74.01; H, 7.23; N, 5.62 .

2-(1-Phenylethylamino)benzonitrile (Table 4, Entry 8) The general procedure was followed with 2-aminobenzonitrile $(118 \mathrm{mg}, 1.00 \mathrm{mmol})$. The reaction mixture was purified by flash column chromatography to give $112 \mathrm{mg}(50 \%)$ of the hydroamination product: ${ }^{1} \mathrm{H}$ NMR (400 $\left.\mathrm{MHz}, \mathrm{CDCl}_{3}, \mathrm{TMS}\right) \delta 1.55(\mathrm{~d}, J=6.8 \mathrm{~Hz}, 3 \mathrm{H}), 4.54\left(\mathrm{dq}, J_{\mathrm{NH}}=4.8 \mathrm{~Hz}, J_{\mathrm{CH}}=6.8 \mathrm{~Hz}, 1 \mathrm{H}\right), 4.92$ $(\mathrm{d}, J=4.8 \mathrm{~Hz}, 1 \mathrm{H}), 6.41$ (d, $J=8.8 \mathrm{~Hz}, 1 \mathrm{H}), 6.58$ (t, $J=6.8 \mathrm{~Hz}, 1 \mathrm{H}), 7.16$ (t, $J=7.2 \mathrm{~Hz}, 2 \mathrm{H})$, 7.20-7.36 (m, 4H); ${ }^{13} \mathrm{C}\left\{{ }^{1} \mathrm{H}\right\}$ NMR (101 MHz, $\left.\mathrm{CDCl}_{3}\right) \delta$ 24.8, 53.1, 95.6, 111.8, 116.5, 117.8, 125.4, 127.2, 128.7, 132.4, 133.9, 143.5, 149.1; Anal. Calcd for $\mathrm{C}_{15} \mathrm{H}_{14} \mathrm{~N}_{2}: \mathrm{C}, 81.05 ; \mathrm{H}, 6.35 ; \mathrm{N}$, 12.60. Found: C, 80.94; H, 6.52; N, 12.36 .

3-(1-Phenylethylamino)benzonitrile (Table 4, Entry 9) The general procedure was followed with 3-aminobenzonitrile $(118 \mathrm{mg}, 1.00 \mathrm{mmol})$. The reaction mixture was purified by flash column chromatography to give $151 \mathrm{mg}(68 \%)$ of the hydroamination product: ${ }^{1} \mathrm{H}$ NMR (400 MHz, $\mathrm{CDCl}_{3}$, TMS) $\delta 1.49(\mathrm{~d}, J=6.0 \mathrm{~Hz}, 3 \mathrm{H}), 4.43(\mathrm{~m}, 2 \mathrm{H}), 6.68(\mathrm{~m}, 2 \mathrm{H}), 6.84(\mathrm{~d}, J=8.0 \mathrm{~Hz}$, 1H), $7.08(\mathrm{~m}, 1 \mathrm{H}), 7.22(\mathrm{~m}, 1 \mathrm{H}), 7.30(\mathrm{~d}, J=4.0 \mathrm{~Hz}, 4 \mathrm{H}) ;{ }^{13} \mathrm{C}\left\{{ }^{1} \mathrm{H}\right\} \mathrm{NMR}\left(101 \mathrm{MHz}, \mathrm{CDCl}_{3}\right) \delta$ 
24.7, 53.1, 115.3, 115.3, 117.5, 119.5, 120.3, 125.5, 127.1, 128.7, 129.6, 143.8, 147.3; Anal. Calcd for $\mathrm{C}_{15} \mathrm{H}_{14} \mathrm{~N}_{2}$ : C, 81.05; H, 6.35; N, 12.60. Found: C, 80.92; H, 6.44; N, 12.42 .

4-(1-Phenylethylamino)benzonitrile (Table 4, Entry 10) The general procedure was followed with 4-aminobenzonitrile $(118 \mathrm{mg}, 1.00 \mathrm{mmol})$. The reaction mixture was purified by flash column chromatography to give $114 \mathrm{mg}(51 \%)$ of the hydroamination product: ${ }^{1} \mathrm{H}$ NMR (500 $\left.\mathrm{MHz}, \mathrm{CDCl}_{3}, \mathrm{TMS}\right) \delta 1.51(\mathrm{~d}, J=6.5 \mathrm{~Hz}, 3 \mathrm{H}), 4.48\left(\mathrm{dq}, J_{\mathrm{NH}}=5.0 \mathrm{~Hz}, J_{\mathrm{CH}}=6.5 \mathrm{~Hz}, 1 \mathrm{H}\right), 4.84$ $(\mathrm{d}, J=5.0 \mathrm{~Hz}, 1 \mathrm{H}), 6.45(\mathrm{dd}, J=6.8,6.5 \mathrm{~Hz}, 2 \mathrm{H}), 7.19-7.32(\mathrm{~m}, 7 \mathrm{H}) ;{ }^{13} \mathrm{C}\left\{{ }^{1} \mathrm{H}\right\} \mathrm{NMR}(126 \mathrm{MHz}$, $\left.\mathrm{CDCl}_{3}\right) \delta$ 24.4, 52.9, 98.1, 112.7, 120.5, 125.5, 127.1, 128.7, 133.3, 143.6, 150.3; Anal. Calcd for $\mathrm{C}_{15} \mathrm{H}_{14} \mathrm{~N}_{2}: \mathrm{C}, 81.05 ; \mathrm{H}, 6.35 ; \mathrm{N}, 12.60$. Found: $\mathrm{C}, 80.94 ; \mathrm{H}, 6.41 ; \mathrm{N}, 12.59$.

Ethyl 2-(1-phenylethylamino)benzoate (Table 4, Entry 11) The general procedure was followed with ethyl 2-aminobenzoate $(165 \mathrm{mg}, 1.00 \mathrm{mmol})$. The reaction mixture was purified by flash column chromatography to give $228 \mathrm{mg}(85 \%)$ of the hydroamination product: ${ }^{1} \mathrm{H}$ NMR (400 MHz, $\left.\mathrm{CDCl}_{3}, \mathrm{TMS}\right) \delta 1.34$ (t, $\left.J=6.8 \mathrm{~Hz}, 3 \mathrm{H}\right), 1.54$ (d, $\left.J=6.4 \mathrm{~Hz}, 3 \mathrm{H}\right), 4.31$ (q, $J=7.2 \mathrm{~Hz}$, $2 \mathrm{H}), 4.52\left(\mathrm{dq}, J_{\mathrm{NH}}=5.6 \mathrm{~Hz}, J_{\mathrm{CH}}=6.4 \mathrm{~Hz}, 1 \mathrm{H}\right), 6.40(\mathrm{~d}, J=8.4 \mathrm{~Hz}, 1 \mathrm{H}), 6.49(\mathrm{~m}, 1 \mathrm{H}), 7.06-7.18$ (m, 2H), $7.25(\mathrm{t}, J=7.6 \mathrm{~Hz}, 2 \mathrm{H}), 7.31(\mathrm{~d}, J=6.8 \mathrm{~Hz}, 2 \mathrm{H}), 7.92(\mathrm{dd}, J=8.0,1.6 \mathrm{~Hz}, 1 \mathrm{H}), 8.26(\mathrm{~d}$, $J=5.6 \mathrm{~Hz}, 1 \mathrm{H}) ;{ }^{13} \mathrm{C}\left\{{ }^{1} \mathrm{H}\right\} \mathrm{NMR}\left(101 \mathrm{MHz}, \mathrm{CDCl}_{3}\right) \delta 14.2,25.0,52.5,60.1,110.1,112.5,114.5$, 125.6, 126.7, 128.5, 131.3, 134.2, 144.8, 150.1, 168.6; Anal. Calcd for $\mathrm{C}_{17} \mathrm{H}_{19} \mathrm{NO}_{2}$ : C, 75.81; $\mathrm{H}$, 7.11; N, 5.20. Found: C, 75.89; H, 7.30; N, 5.19.

Ethyl 3-(1-phenylethylamino)benzoate (Table 4, Entry 12) The general procedure was followed with ethyl 3-aminobenzoate $(165 \mathrm{mg}, 1.00 \mathrm{mmol})$. The reaction mixture was purified by flash column chromatography to give $189 \mathrm{mg}(70 \%)$ of the hydroamination product: ${ }^{1} \mathrm{H}$ NMR (400 MHz, $\mathrm{CDCl}_{3}$, TMS) $\delta 1.29$ (t, $\left.J=6.8 \mathrm{~Hz}, 3 \mathrm{H}\right), 1.45$ (d, $J=6.8 \mathrm{~Hz}, 3 \mathrm{H}$ ), 4.27 (q, $J=7.2 \mathrm{~Hz}$, 2H), $4.33(\mathrm{~s}, 1 \mathrm{H}), 4.47$ (q, $J=6.4 \mathrm{~Hz}, 1 \mathrm{H}), 6.60(\mathrm{dd}, J=8.2,2.0 \mathrm{~Hz}, 1 \mathrm{H}), 7.07$ (t, $J=8.0 \mathrm{~Hz}$, 1H), $7.17(\mathrm{~m}, 1 \mathrm{H}), 7.22-7.34(\mathrm{~m}, 6 \mathrm{H}) ;{ }^{13} \mathrm{C}\left\{{ }^{1} \mathrm{H}\right\}$ NMR $\left(101 \mathrm{MHz}, \mathrm{CDCl}_{3}\right) \delta$ 14.1, 24.6, 53.1, 60.1, 114.2, 117.0, 117.9, 125.6, 126.7, 128.4, 128.8, 130.9, 144.6, 147.1, 166.8; Anal. Calcd for $\mathrm{C}_{17} \mathrm{H}_{19} \mathrm{NO}_{2}: \mathrm{C}, 75.81 ; \mathrm{H}, 7.11 ; \mathrm{N}, 5.20$. Found: C, 75.78; H, 7.23; N, 5.03.

Ethyl 4-(1-phenylethylamino)benzoate (Table 4, Entry 13) The general procedure excluding the trifluoromethanesulfonic acid cocatalyst was followed with ethyl 4-aminobenzoate (165 mg, $1.00 \mathrm{mmol})$. The reaction mixture was purified by flash column chromatography to give $241 \mathrm{mg}$ (89\%) of the hydroamination product: ${ }^{1} \mathrm{H}$ NMR (500 MHz, $\mathrm{CDCl}_{3}$, TMS) $\delta 1.28$ (t, $J=7.0 \mathrm{~Hz}$, $3 \mathrm{H}), 1.46(\mathrm{~d}, J=6.5 \mathrm{~Hz}, 3 \mathrm{H}), 4.25(\mathrm{q}, J=7.0 \mathrm{~Hz}, 2 \mathrm{H}), 4.49\left(\mathrm{dq}, J_{\mathrm{NH}}=5.5 \mathrm{~Hz}, J_{\mathrm{CH}}=6.5 \mathrm{~Hz}\right.$, 
1H), $4.74(\mathrm{~d}, J=5.5 \mathrm{~Hz}, 1 \mathrm{H}), 6.45(\mathrm{~d}, J=9.0 \mathrm{~Hz}, 2 \mathrm{H}), 7.18(\mathrm{~m}, 1 \mathrm{H}), 7.21-7.30(\mathrm{~m}, 4 \mathrm{H}), 7.80(\mathrm{~d}$, $J=8.5 \mathrm{~Hz}, 2 \mathrm{H}) ;{ }^{13} \mathrm{C}\left\{{ }^{1} \mathrm{H}\right\} \operatorname{NMR}\left(126 \mathrm{MHz}, \mathrm{CDCl}_{3}\right) \delta 14.2,24.4,52.8,59.9,111.9,118.2,125.5$, 126.8, 128.5, 131.1, 144.1, 150.9, 166.7; Anal. Calcd for $\mathrm{C}_{17} \mathrm{H}_{19} \mathrm{NO}_{2}$ : C, 75.81; H, 7.11; N, 5.20. Found: C, 75.47; H, 7.38; N, 5.08.

$N$-(3-(1-phenylethylamino)phenyl)acetamide (Table 4, Entry 14) The general procedure excluding the trifluoromethanesulfonic acid cocatalyst was followed with $\mathrm{N}$-(3aminophenyl)acetamide $(150 \mathrm{mg}, 1.00 \mathrm{mmol})$. The reaction mixture was purified by flash column chromatography to give $231 \mathrm{mg}(91 \%)$ of the hydroamination product: ${ }^{1} \mathrm{H}$ NMR (400 $\left.\mathrm{MHz}, \mathrm{CDCl}_{3}, \mathrm{TMS}\right) \delta 1.48$ (d, $\left.J=6.8 \mathrm{~Hz}, 3 \mathrm{H}\right), 2.07$ (s, 3H), 4.16 (q, $\left.J=7.2 \mathrm{~Hz}, 1 \mathrm{H}\right), 4.47$ (q, $J$ $=6.6 \mathrm{~Hz}, 1 \mathrm{H}), 6.25(\mathrm{~d}, J=8.0 \mathrm{~Hz}, 1 \mathrm{H}), 6.71(\mathrm{~d}, J=7.6 \mathrm{~Hz}, 1 \mathrm{H}), 7.01(\mathrm{t}, J=8.0 \mathrm{~Hz}, 2 \mathrm{H}), 7.18-$ $7.40(\mathrm{~m}, 5 \mathrm{H}), 7.88(\mathrm{~s}, 1 \mathrm{H}) ;{ }^{13} \mathrm{C}\left\{{ }^{1} \mathrm{H}\right\}$ NMR $\left(101 \mathrm{MHz}, \mathrm{CDCl}_{3}\right) \delta$ 24.4, 24.8, 53.2, 104.8, 108.6, 108.9, 125.7, 126.7, 128.5, 129.3, 138.8, 145.0, 147.8, 168.7; Anal. Calcd for $\mathrm{C}_{16} \mathrm{H}_{18} \mathrm{~N}_{2} \mathrm{O}: \mathrm{C}$, 75.56; H, 7.13; N, 11.01. Found: C, 75.28; H, 7.18; N, 10.73.

4-(1-phenylethylamino)benzamide $\cdot 0.25 \mathrm{H}_{2} \mathrm{O}$ (Table 4, Entry 15) The general procedure excluding the trifluoromethanesulfonic acid cocatalyst was followed with 4-aminobenzamide $(136 \mathrm{mg}, 1.00 \mathrm{mmol})$. The reaction mixture was purified by flash column chromatography to give $208 \mathrm{mg}$ (89\%) of the hydroamination product: ${ }^{1} \mathrm{H}$ NMR (400 MHz, DMSO-d 6 , TMS) $\delta 1.42$ $(\mathrm{d}, J=6.4 \mathrm{~Hz}, 3 \mathrm{H}), 4.53\left(\mathrm{dq}, J_{\mathrm{NH}}=5.5 \mathrm{~Hz}, J_{\mathrm{CH}}=6.4 \mathrm{~Hz}, 1 \mathrm{H}\right), 6.49(\mathrm{~d}, J=8.8 \mathrm{~Hz}, 2 \mathrm{H}), 6.70(\mathrm{~d}$, $J=7.2 \mathrm{~Hz}, 1 \mathrm{H}), 6.85$ (br s, $1 \mathrm{H}), 7.17$ (t, $J=7.0 \mathrm{~Hz}, 1 \mathrm{H}), 7.28$ (t, $J=7.6 \mathrm{~Hz}, 2 \mathrm{H}), 7.35$ (d, $J=7.6$ $\mathrm{Hz}, 2 \mathrm{H}), 7.49$ (br s, 2H), $7.56(\mathrm{~d}, J=8.8 \mathrm{~Hz}, 1 \mathrm{H}) ;{ }^{1} \mathrm{H} \mathrm{NMR}\left(500 \mathrm{MHz}, \mathrm{CD}_{2} \mathrm{Cl}_{2}\right) \delta 0.50$ (br s, $\left.H_{2} \mathrm{O}, 0.5 \mathrm{H}\right), 1.53(\mathrm{~d}, J=6.5 \mathrm{~Hz}, 3 \mathrm{H}), 4.55\left(\mathrm{~m}, \mathrm{NH}\right.$ and $\left.\mathrm{CHCH}_{3}, 2 \mathrm{H}\right), 5.59(\mathrm{br} \mathrm{s}, 2 \mathrm{H}), 6.51$ (d, $J=$ $8.5 \mathrm{~Hz}, 2 \mathrm{H}), 7.24(\mathrm{~m}, 1 \mathrm{H}), 7.30-7.38(\mathrm{~m}, 4 \mathrm{H}), 7.54(\mathrm{~d}, J=9.0 \mathrm{~Hz}, 2 \mathrm{H}) ;{ }^{13} \mathrm{C}\left\{{ }^{1} \mathrm{H}\right\} \operatorname{NMR}(101$ MHz, DMSO-d $\left.{ }_{6}\right) \delta 24.4,51.8,111.5,121.0,125.8,126.5,128.4,128.8,145.6,150.4,168.0$; Anal. Calcd for $\mathrm{C}_{15} \mathrm{H}_{16.5} \mathrm{~N}_{2} \mathrm{O}_{1.25}$ : C, 73.59; H, 6.79; N, 11.44. Found: C, 73.41; H, 6.59; N, 11.30.

Determination of the Rate constants $\left(k_{\mathrm{obs}}\right)$ for the Nucleophilic Attack of Aniline and Substituted Anilines on Benzylpalladium Complexes. Benzylpalladium complexes (4-10) $(0.0108 \mathrm{mmol}), \mathrm{PPh}_{3}(0.0108 \mathrm{mmol})$, and 1,3,5-trimethylbenzene $(0.00719 \mathrm{mmol}$, internal standard) were dissolved in a mixture of $\mathrm{CD}_{2} \mathrm{Cl}_{2}$ and THF- $d_{8}(0.2 \mathrm{~mL}: 0.4 \mathrm{~mL})$, and the solution was transferred to a screw-capped NMR tube. The arylamines $(0.054 \mathrm{mmol})$ in $0.2 \mathrm{~mL}$ THF- $d_{8}$ were added by syringe into the NMR tube immediately before the tube was placed in a probe at the appropriate temperature. Decay of the $\mathrm{CH}_{2} \mathrm{Ph}$ resonance was monitored by ${ }^{1} \mathrm{H}$ NMR 
spectroscopy over at least three half-lives with an automated data collection program. Kinetic data were fit to the expression $y=m_{1}+m_{2} \exp ^{-k t}$, in which $k$ is the first-order rate constant $k_{\mathrm{obs}}$.

Determination of the Rate constants ( $k_{\text {obs }}$ ) for the Nucleophilic Attack of Aniline and Substituted Anilines on Allylpalladium Complexes. Allylpalladium complexes (1, 2, 11-15) (0.0197 mmol), diphenylacetylene $(0.296 \mathrm{mmol})$, and a capillary containing $\mathrm{PPh}_{3}$ in tetrahydrofuran were loaded into a screw-capped NMR tube, followed by $0.50 \mathrm{~mL} \mathrm{CH}_{2} \mathrm{Cl}_{2}$. Aniline $(1.98 \mathrm{mmol})$ was added by syringe into the NMR tube immediately before it was placed in a probe at the appropriate temperature. Decay of the allylpalladium complexes were monitored by ${ }^{31} \mathrm{P}$ NMR spectroscopy over at least three half-lives with an automated data collection program. Kinetic data were fit to the expression $y=m_{1}+m_{2} \exp ^{-k t}$, in which $k$ is the first-order rate constant $k_{\text {obs. }}$.

Determination of the Rate constants $\left(k_{\text {obs }}\right)$ for the Nucleophilic Attack of Aniline on 1,1Dimethylallylpalladium Complexes. 1,1-dimethylallylpalladium complexes (19-22) (0.0197 mmol), diphenylacetylene $(0.296 \mathrm{mmol})$, and a capillary containing $\mathrm{PPh}_{3}$ in tetrahydrofuran were loaded into a screw-capped NMR tube, followed by 0.50 or $0.17 \mathrm{~mL} \mathrm{CH}_{2} \mathrm{Cl}_{2}$. Aniline (30 $\mu$, 0.295 or $360 \mu 1,3.95 \mathrm{mmol}$ ) was added by syringe into the NMR tube immediately before it was placed in a probe at the appropriate temperature. Decay of the 1,1-dimethylallylpalladium complexes was monitored by ${ }^{31} \mathrm{P}$ NMR spectroscopy over at least three half-lives with an automated data collection program. Kinetic data were fit to the expression $y=m_{1}+m_{2} \exp ^{-k t}$, in which $k$ is the first-order rate constant $k_{\mathrm{obs}}$. 


\section{References}

(1) Palenik, R. C.; Palenik, G. J. Syn. React. Inorg. Met. 1992, 22, 1395-1399.

(2) Drew, D.; Doyle, J. R. Inorg. Synth. 1990, 28, 346-349.

(3) White, D. A. Synth. Inorg. Met.-Org. Chem. 1971, 1, 133-9.

(4) De Graaf, W.; Boersma, J.; Van Koten, G. Organometallics 1990, 9, 1479-84.

(5) Trost, B. M.; Strege, P. E.; Weber, L.; Fullerton, T. J.; Dietsche, T. J. J. Am. Chem. Soc. 1978, 100, 3407-15.

(6) Auburn, P. R.; Mackenzie, P. B.; Bosnich, B. J. Am. Chem. Soc. 1985, 107, 2033-46.

(7) Stockland, R.; Anderson, G.; Rath, N.; Braddock-Wilking, J.; Ellegood, J. Can. J. Chem. 1996, 74, 1990-1997.

(8) Paonessa, R. S.; Prignano, A. L.; Trogler, W. C. Organometallics 1985, 4, 647-657. 


\section{Experimental Procedure for the X-Ray Diffraction of 1}

\section{Data Collection}

A yellow plate crystal of $\mathrm{C}_{42} \mathrm{H}_{37} \mathrm{ClOP}_{2} \mathrm{Pd} \cdot \mathrm{C}_{6} \mathrm{H}_{6}, \mathrm{C}_{48} \mathrm{H}_{43} \mathrm{ClOP}_{2} \mathrm{Pd}$, having approximate dimensions of $0.20 \times 0.15 \times 0.08 \mathrm{~mm}^{3}$ was mounted with epoxy cement on the tip of a fine glass fiber. All measurements were made on a Nonius KappaCCD diffractometer with graphite monochromated Mo-K $\alpha$ radiation.

Cell constants and an orientation matrix for data collection corresponded to a primitive monoclinic cell with dimensions:

$$
\begin{array}{ll}
\mathrm{a}=11.874(2) \AA & \alpha=90^{\circ} \\
\mathrm{b}=15.938(3) \AA & \beta=93.14(3) \mathrm{o} \\
\mathrm{c}=21.337(4) \AA & \gamma=90^{\circ} \\
\mathrm{V}=4031.9(14) \AA^{3} &
\end{array}
$$

For $Z=4$ and F.W. $=839.61$, the calculated density is $1.383 \mathrm{~g} / \mathrm{cm}^{3}$. Based on a statistical analysis of intensity distribution, and the successful solution and refinement of the structure, the space group was determined to be $P 2_{1} / c$ (\#14).

The data were collected at a temperature of $173(2) \mathrm{K}$ to a maximum $2 \theta$ value of $56.62^{\circ}$. Four omega scans consisting of 58, 45, 53, and 22 data frames, respectively, were collected with a frame width of $1.30^{\circ}$ and a detector-to-crystal distance, Dx, of $35.0 \mathrm{~mm}$. Each frame was exposed twice (for the purpose of de-zingering) for a total of $52 \mathrm{~s}$. The data frames were processed and scaled using the DENZO software package. ${ }^{1}$

\section{$\underline{\text { Data Reduction }}$}

A total of 15033 reflections were collected of which 9948 were unique and observed $\left(\mathrm{R}_{\text {int }}\right.$ $=0.0541)$. The linear absorption coefficient, $\mu$, for Mo-K $\alpha$ radiation is $6.42 \mathrm{~cm}^{-1}$, and no absorption correction was applied. The data were corrected for Lorentz and polarization effects.

\section{$\underline{\text { Structure Solution and Refinement }}$}

The structure was solved by direct methods and expanded using Fourier techniques ${ }^{2}$. The non-hydrogen atoms were refined anisotropically, and hydrogen atoms were treated as idealized contributions. The final cycle of full-matrix least-squares refinement ${ }^{3}$ on $\mathrm{F}$ was based on 9948 observed reflections (I $>2.00 \sigma(\mathrm{I})$ ) and 478 variable parameters and converged with unweighted and weighted agreement factors of:

$$
\begin{gathered}
\mathrm{R}=\Sigma|| \mathrm{Fo}|-| \mathrm{Fc} \| / \Sigma|\mathrm{Fo}|=0.0543 \\
\mathrm{R}_{\mathrm{W}}=\left\{\Sigma\left[\mathrm{w}\left(\mathrm{F}_{\mathrm{o}}{ }^{2}-\mathrm{F}_{\mathrm{c}}{ }^{2}\right)^{2}\right] / \Sigma\left[\mathrm{w}\left(\mathrm{F}_{\mathrm{o}}{ }^{2}\right)^{2}\right]\right\}^{1 / 2}=0.0941
\end{gathered}
$$


The maximum and minimum peaks on the final difference Fourier map corresponded to 0.559 and $-0.569 \mathrm{e}^{-} / \AA^{3}$ respectively.

\section{REFERENCES}

(1) Z. Otwinowski and W. Minor, "Processing of X-Ray Diffraction Data Collected in Oscillation Mode," Methods in Enzymology, vol. 276: Macromolecular Crystallography, part A, 307-326, 1997, C.W. Carter, Jr. \& R.M. Sweet, Eds., Academic Press.

(2) Acta Cryst. A46 (1990) 467-473

(3) Least Squares function minimized:

$$
\Sigma w\left(\mathrm{~F}_{\mathrm{o}}^{2}-\mathrm{F}_{\mathrm{c}}^{2}\right)^{2}
$$




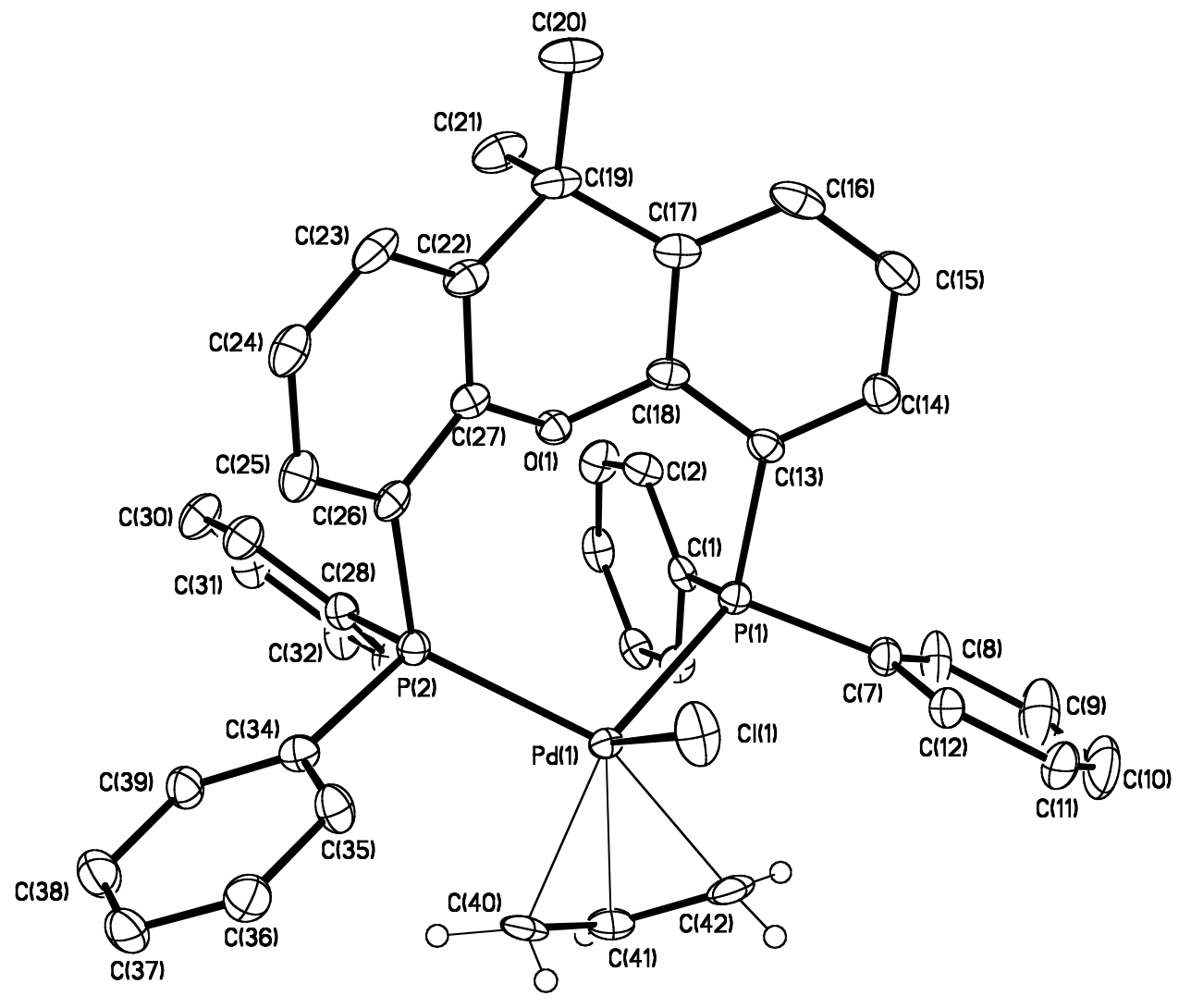

Figure S4. ORTEP diagram of 1 
Table S1. Crystal data and structure refinement for 1.

Empirical formula

Formula weight

Temperature

Wavelength

Crystal system

Space group

Unit cell dimensions

Volume

Z

Density (calculated)

Absorption coefficient

$\mathrm{F}(000)$

Crystal size

Theta range for data collection

Index ranges

Reflections collected

Independent reflections

Completeness to theta $=28.31^{\circ}$

Absorption correction

Max. and min. transmission

Refinement method

Data / restraints / parameters

Goodness-of-fit on $\mathrm{F}^{2}$

Final $\mathrm{R}$ indices [I $>2 \operatorname{sigma}(\mathrm{I})]$

$\mathrm{R}$ indices (all data)

Largest diff. peak and hole
$\mathrm{C}_{48} \mathrm{H}_{43} \mathrm{Cl} \mathrm{O} \mathrm{P}_{2} \mathrm{Pd}$

839.61

173(2) K

$0.71073 \AA$

Monoclinic

$\mathrm{P} 2(1) / \mathrm{c}$

$\mathrm{a}=11.874(2) \AA \quad \alpha=90^{\circ}$.

$\mathrm{b}=15.938(3) \AA \quad \beta=93.14(3)^{\circ}$.

$\mathrm{c}=21.337(4) \AA \quad \gamma=90^{\circ}$.

4031.9(14) $\AA^{3}$

4

$1.383 \mathrm{~g} / \mathrm{cm}^{3}$

$6.42 \mathrm{~cm}^{-1}$

1728

$0.20 \times 0.15 \times 0.08 \mathrm{~mm}^{3}$

2.30 to $28.31^{\circ}$.

$-15<=\mathrm{h}<=15,-21<=\mathrm{k}<=15,-28<=\mathrm{l}<=28$

15033

$9948[\mathrm{R}(\mathrm{int})=0.0541]$

$99.1 \%$

None

0.9504 and 0.8824

Full-matrix least-squares on $\mathrm{F}^{2}$

9948 / 0 / 478

0.996

$\mathrm{R} 1=0.0543, \mathrm{wR} 2=0.0941$

$\mathrm{R} 1=0.1294, \mathrm{wR} 2=0.1120$

0.559 and -0.569 e. $\AA^{-3}$ 
Table S2. Atomic coordinates ( $x \mathbf{1 0}^{4}$ ) and equivalent isotropic displacement parameters $\left(\AA^{2} \mathbf{x} \mathbf{1 0}^{3}\right)$ for 1 . U(eq) is defined as one third of the trace of the orthogonalized Uij tensor.

\begin{tabular}{|c|c|c|c|c|}
\hline & $\mathrm{x}$ & $\mathrm{y}$ & $\mathrm{z}$ & $\mathrm{U}(\mathrm{eq})$ \\
\hline $\operatorname{Pd}(1)$ & $8270(1)$ & $2447(1)$ & $906(1)$ & $23(1)$ \\
\hline $\mathrm{Cl}(1)$ & $8314(1)$ & $1590(1)$ & $-221(1)$ & $45(1)$ \\
\hline $\mathrm{P}(1)$ & $7524(1)$ & $1362(1)$ & $1513(1)$ & $22(1)$ \\
\hline $\mathrm{P}(2)$ & $6782(1)$ & $3356(1)$ & $598(1)$ & $23(1)$ \\
\hline $\mathrm{O}(1)$ & $5400(2)$ & $1996(2)$ & 994(1) & $24(1)$ \\
\hline $\mathrm{C}(1)$ & $7186(3)$ & $1795(2)$ & $2272(2)$ & $23(1)$ \\
\hline$C(2)$ & $6083(3)$ & $1822(2)$ & $2468(2)$ & $29(1)$ \\
\hline$C(3)$ & $5846(4)$ & $2226(3)$ & $3022(2)$ & $38(1)$ \\
\hline$C(4)$ & $6695(4)$ & $2572(2)$ & $3399(2)$ & $35(1)$ \\
\hline$C(5)$ & $7798(4)$ & $2540(2)$ & $3216(2)$ & $31(1)$ \\
\hline$C(6)$ & $8037(4)$ & $2164(2)$ & $2651(2)$ & $30(1)$ \\
\hline$C(7)$ & $8584(3)$ & $560(2)$ & $1692(2)$ & $28(1)$ \\
\hline$C(8)$ & $8895(4)$ & $303(3)$ & $2292(2)$ & $48(1)$ \\
\hline $\mathrm{C}(9)$ & $9778(5)$ & $-241(3)$ & $2402(3)$ & $74(2)$ \\
\hline$C(10)$ & $10352(5)$ & $-549(3)$ & 1916(3) & $77(2)$ \\
\hline$C(11)$ & $10034(4)$ & $-328(3)$ & $1300(3)$ & $56(1)$ \\
\hline$C(12)$ & $9147(3)$ & $228(2)$ & 1192(2) & $36(1)$ \\
\hline$C(13)$ & $6298(3)$ & $727(2)$ & $1277(2)$ & $25(1)$ \\
\hline$C(14)$ & $6246(4)$ & $-142(2)$ & $1324(2)$ & $30(1)$ \\
\hline$C(15)$ & $5282(4)$ & $-563(3)$ & $1106(2)$ & $40(1)$ \\
\hline$C(16)$ & $4350(4)$ & $-144(3)$ & $843(2)$ & $37(1)$ \\
\hline$C(17)$ & $4358(3)$ & $722(2)$ & $799(2)$ & $28(1)$ \\
\hline$C(18)$ & $5342(3)$ & $1129(2)$ & $1020(2)$ & $25(1)$ \\
\hline$C(19)$ & $3397(3)$ & $1273(3)$ & $558(2)$ & $34(1)$ \\
\hline$C(20)$ & $2490(3)$ & $800(3)$ & $164(2)$ & $47(1)$ \\
\hline $\mathrm{C}(21)$ & $2842(3)$ & $1668(3)$ & $1133(2)$ & $44(1)$ \\
\hline$C(22)$ & $3921(3)$ & $1988(2)$ & $194(2)$ & $29(1)$ \\
\hline $\mathrm{C}(23)$ & $3479(3)$ & $2332(3)$ & $-368(2)$ & $34(1)$ \\
\hline $\mathrm{C}(24)$ & $4016(4)$ & 2997(3) & $-643(2)$ & $34(1)$ \\
\hline$C(25)$ & $5007(4)$ & $3328(2)$ & $-376(2)$ & $32(1)$ \\
\hline
\end{tabular}




\begin{tabular}{|c|c|c|c|c|}
\hline$C(26)$ & $5485(3)$ & 2991(2) & $188(2)$ & $25(1)$ \\
\hline$C(27)$ & $4912(3)$ & $2326(2)$ & $441(2)$ & $23(1)$ \\
\hline$C(28)$ & $6247(3)$ & $3947(2)$ & $1254(2)$ & $25(1)$ \\
\hline$C(29)$ & $5246(3)$ & $4397(3)$ & 1197(2) & $36(1)$ \\
\hline$C(30)$ & $4873(4)$ & $4834(3)$ & $1705(2)$ & $42(1)$ \\
\hline $\mathrm{C}(31)$ & $5476(4)$ & $4822(3)$ & $2269(2)$ & $41(1)$ \\
\hline$C(32)$ & $6474(4)$ & $4377(3)$ & $2331(2)$ & $41(1)$ \\
\hline C(33) & $6857(3)$ & $3945(2)$ & $1825(2)$ & $32(1)$ \\
\hline$C(34)$ & $7240(3)$ & $4157(2)$ & $53(2)$ & $27(1)$ \\
\hline$C(35)$ & $7738(4)$ & $3864(2)$ & $-485(2)$ & $33(1)$ \\
\hline$C(36)$ & $8109(4)$ & $4431(3)$ & $-921(2)$ & $37(1)$ \\
\hline$C(37)$ & $8008(4)$ & $5274(3)$ & $-830(2)$ & $39(1)$ \\
\hline$C(38)$ & $7537(4)$ & $5576(3)$ & $-290(2)$ & $42(1)$ \\
\hline C(39) & $7156(3)$ & $5012(2)$ & $149(2)$ & $32(1)$ \\
\hline$C(40)$ & $9521(3)$ & $3364(3)$ & $645(2)$ & $45(1)$ \\
\hline$C(41)$ & $9901(3)$ & $2956(3)$ & $1200(2)$ & $40(1)$ \\
\hline$C(42)$ & $9992(3)$ & $2078(3)$ & $1185(2)$ & $38(1)$ \\
\hline$C(43)$ & $12057(8)$ & $1433(7)$ & $2908(4)$ & $131(4)$ \\
\hline$C(44)$ & $12048(7)$ & $2290(5)$ & $2807(4)$ & 111(3) \\
\hline$C(45)$ & 11491(7) & $2705(5)$ & 3191(3) & $103(2)$ \\
\hline$C(46)$ & $10888(6)$ & $2393(6)$ & $3684(4)$ & $103(3)$ \\
\hline$C(47)$ & $10924(6)$ & $1549(6)$ & $3790(4)$ & $98(2)$ \\
\hline$C(48)$ & $11539(8)$ & $1084(6)$ & $3402(4)$ & $118(3)$ \\
\hline
\end{tabular}


Table S3. Bond lengths $[\AA ̊]$ and angles $\left[{ }^{\circ}\right]$ for 1 .

\begin{tabular}{|c|c|c|c|}
\hline $\operatorname{Pd}(1)-C(41)$ & $2.160(4)$ & $C(19)-C(22)$ & $1.530(5)$ \\
\hline $\operatorname{Pd}(1)-C(40)$ & $2.178(4)$ & $C(19)-C(21)$ & $1.557(5)$ \\
\hline $\operatorname{Pd}(1)-C(42)$ & $2.179(4)$ & $C(22)-C(27)$ & $1.373(5)$ \\
\hline $\mathrm{Pd}(1)-\mathrm{P}(2)$ & $2.3502(11)$ & $C(22)-C(23)$ & $1.394(5)$ \\
\hline $\mathrm{Pd}(1)-\mathrm{P}(1)$ & $2.3623(10)$ & $C(23)-C(24)$ & $1.383(6)$ \\
\hline $\mathrm{Pd}(1)-\mathrm{Cl}(1)$ & $2.7694(10)$ & $C(24)-C(25)$ & $1.384(5)$ \\
\hline$P(1)-C(7)$ & $1.819(4)$ & $C(25)-C(26)$ & $1.409(5)$ \\
\hline $\mathrm{P}(1)-\mathrm{C}(13)$ & $1.821(4)$ & $C(26)-C(27)$ & $1.384(5)$ \\
\hline$P(1)-C(1)$ & $1.825(3)$ & $\mathrm{C}(28)-\mathrm{C}(33)$ & $1.383(5)$ \\
\hline$P(2)-C(26)$ & $1.824(4)$ & $\mathrm{C}(28)-\mathrm{C}(29)$ & $1.388(5)$ \\
\hline $\mathrm{P}(2)-\mathrm{C}(34)$ & $1.829(4)$ & $\mathrm{C}(29)-\mathrm{C}(30)$ & $1.383(5)$ \\
\hline $\mathrm{P}(2)-\mathrm{C}(28)$ & $1.830(4)$ & $\mathrm{C}(30)-\mathrm{C}(31)$ & $1.365(5)$ \\
\hline $\mathrm{O}(1)-\mathrm{C}(18)$ & $1.384(4)$ & $C(31)-C(32)$ & $1.381(6)$ \\
\hline $\mathrm{O}(1)-\mathrm{C}(27)$ & $1.388(4)$ & $\mathrm{C}(32)-\mathrm{C}(33)$ & $1.379(5)$ \\
\hline$C(1)-C(6)$ & $1.389(5)$ & $\mathrm{C}(34)-\mathrm{C}(39)$ & $1.382(5)$ \\
\hline$C(1)-C(2)$ & $1.397(5)$ & $\mathrm{C}(34)-\mathrm{C}(35)$ & $1.401(5)$ \\
\hline$C(2)-C(3)$ & $1.390(5)$ & $C(35)-C(36)$ & $1.387(5)$ \\
\hline$C(3)-C(4)$ & $1.370(6)$ & $C(36)-C(37)$ & $1.365(5)$ \\
\hline$C(4)-C(5)$ & $1.388(5)$ & $\mathrm{C}(37)-\mathrm{C}(38)$ & $1.393(5)$ \\
\hline$C(5)-C(6)$ & $1.389(5)$ & $\mathrm{C}(38)-\mathrm{C}(39)$ & $1.390(5)$ \\
\hline$C(7)-C(8)$ & $1.376(5)$ & $C(40)-C(41)$ & $1.403(5)$ \\
\hline$C(7)-C(12)$ & $1.393(5)$ & $C(41)-C(42)$ & $1.403(6)$ \\
\hline $\mathrm{C}(8)-\mathrm{C}(9)$ & $1.371(6)$ & $\mathrm{C}(43)-\mathrm{C}(48)$ & $1.367(11)$ \\
\hline$C(9)-C(10)$ & $1.364(7)$ & $C(43)-C(44)$ & $1.382(10)$ \\
\hline $\mathrm{C}(10)-\mathrm{C}(11)$ & $1.393(7)$ & $C(44)-C(45)$ & $1.266(9)$ \\
\hline$C(11)-C(12)$ & $1.387(6)$ & $C(45)-C(46)$ & $1.397(9)$ \\
\hline$C(13)-C(18)$ & $1.389(5)$ & $C(46)-C(47)$ & $1.365(9)$ \\
\hline$C(13)-C(14)$ & $1.390(5)$ & $C(47)-C(48)$ & $1.354(10)$ \\
\hline$C(14)-C(15)$ & $1.385(5)$ & & \\
\hline$C(15)-C(16)$ & $1.385(6)$ & $C(41)-P d(1)-C(40)$ & $37.73(15)$ \\
\hline$C(16)-C(17)$ & $1.383(5)$ & $C(41)-\operatorname{Pd}(1)-C(42)$ & $37.73(15)$ \\
\hline$C(17)-C(18)$ & $1.396(5)$ & $C(40)-P d(1)-C(42)$ & $66.91(16)$ \\
\hline $\mathrm{C}(17)-\mathrm{C}(19)$ & $1.509(5)$ & $\mathrm{C}(41)-\mathrm{Pd}(1)-\mathrm{P}(2)$ & $119.88(12)$ \\
\hline$C(19)-C(20)$ & $1.528(5)$ & $\mathrm{C}(40)-\mathrm{Pd}(1)-\mathrm{P}(2)$ & $91.58(12)$ \\
\hline
\end{tabular}




\begin{tabular}{|c|c|c|c|}
\hline$C(42)-P d(1)-P(2)$ & $157.29(12)$ & $\mathrm{C}(9)-\mathrm{C}(10)-\mathrm{C}(11)$ & $120.4(5)$ \\
\hline $\mathrm{C}(41)-\mathrm{Pd}(1)-\mathrm{P}(1)$ & $118.22(12)$ & $C(12)-C(11)-C(10)$ & $118.8(4)$ \\
\hline $\mathrm{C}(40)-\mathrm{Pd}(1)-\mathrm{P}(1)$ & $155.92(12)$ & $C(11)-C(12)-C(7)$ & $120.6(4)$ \\
\hline $\mathrm{C}(42)-\mathrm{Pd}(1)-\mathrm{P}(1)$ & $91.55(11)$ & $\mathrm{C}(18)-\mathrm{C}(13)-\mathrm{C}(14)$ & $116.7(4)$ \\
\hline $\mathrm{P}(2)-\mathrm{Pd}(1)-\mathrm{P}(1)$ & $107.83(4)$ & $\mathrm{C}(18)-\mathrm{C}(13)-\mathrm{P}(1)$ & $118.4(3)$ \\
\hline $\mathrm{C}(41)-\mathrm{Pd}(1)-\mathrm{Cl}(1)$ & $112.18(12)$ & $\mathrm{C}(14)-\mathrm{C}(13)-\mathrm{P}(1)$ & $124.8(3)$ \\
\hline $\mathrm{C}(40)-\mathrm{Pd}(1)-\mathrm{Cl}(1)$ & $93.62(13)$ & $C(15)-C(14)-C(13)$ & $119.8(4)$ \\
\hline$C(42)-\operatorname{Pd}(1)-C l(1)$ & $92.41(12)$ & $C(16)-C(15)-C(14)$ & $122.1(4)$ \\
\hline $\mathrm{P}(2)-\mathrm{Pd}(1)-\mathrm{Cl}(1)$ & $96.33(4)$ & $C(17)-C(16)-C(15)$ & $119.9(4)$ \\
\hline $\mathrm{P}(1)-\mathrm{Pd}(1)-\mathrm{Cl}(1)$ & $98.09(4)$ & $C(16)-C(17)-C(18)$ & $116.8(4)$ \\
\hline$C(7)-P(1)-C(13)$ & 101.61(18) & $C(16)-C(17)-C(19)$ & $126.6(4)$ \\
\hline $\mathrm{C}(7)-\mathrm{P}(1)-\mathrm{C}(1)$ & $105.26(16)$ & $\mathrm{C}(18)-\mathrm{C}(17)-\mathrm{C}(19)$ & $116.6(3)$ \\
\hline$C(13)-P(1)-C(1)$ & $104.17(16)$ & $\mathrm{O}(1)-\mathrm{C}(18)-\mathrm{C}(13)$ & $115.8(3)$ \\
\hline$C(7)-P(1)-P d(1)$ & $110.68(13)$ & $\mathrm{O}(1)-\mathrm{C}(18)-\mathrm{C}(17)$ & $119.5(4)$ \\
\hline$C(13)-P(1)-P d(1)$ & $124.93(12)$ & $C(13)-C(18)-C(17)$ & $124.7(4)$ \\
\hline$C(1)-P(1)-P d(1)$ & $108.48(12)$ & $C(17)-C(19)-C(20)$ & $113.4(4)$ \\
\hline $\mathrm{C}(26)-\mathrm{P}(2)-\mathrm{C}(34)$ & 101.01(17) & $C(17)-C(19)-C(22)$ & $106.6(3)$ \\
\hline $\mathrm{C}(26)-\mathrm{P}(2)-\mathrm{C}(28)$ & $102.22(17)$ & $C(20)-C(19)-C(22)$ & $112.5(3)$ \\
\hline $\mathrm{C}(34)-\mathrm{P}(2)-\mathrm{C}(28)$ & $104.70(16)$ & $C(17)-C(19)-C(21)$ & $108.1(3)$ \\
\hline $\mathrm{C}(26)-\mathrm{P}(2)-\mathrm{Pd}(1)$ & $122.66(12)$ & $C(20)-C(19)-C(21)$ & $108.5(3)$ \\
\hline $\mathrm{C}(34)-\mathrm{P}(2)-\mathrm{Pd}(1)$ & 111.29(13) & $C(22)-C(19)-C(21)$ & $107.5(3)$ \\
\hline $\mathrm{C}(28)-\mathrm{P}(2)-\mathrm{Pd}(1)$ & $112.96(13)$ & $C(27)-C(22)-C(23)$ & $116.8(4)$ \\
\hline $\mathrm{C}(18)-\mathrm{O}(1)-\mathrm{C}(27)$ & $113.2(3)$ & $C(27)-C(22)-C(19)$ & $117.5(3)$ \\
\hline$C(6)-C(1)-C(2)$ & $118.5(3)$ & $C(23)-C(22)-C(19)$ & $125.8(4)$ \\
\hline $\mathrm{C}(6)-\mathrm{C}(1)-\mathrm{P}(1)$ & 119.2(3) & $\mathrm{C}(24)-\mathrm{C}(23)-\mathrm{C}(22)$ & $120.4(4)$ \\
\hline $\mathrm{C}(2)-\mathrm{C}(1)-\mathrm{P}(1)$ & $122.1(3)$ & $C(23)-C(24)-C(25)$ & $121.3(4)$ \\
\hline $\mathrm{C}(3)-\mathrm{C}(2)-\mathrm{C}(1)$ & $120.4(4)$ & $C(24)-C(25)-C(26)$ & $119.9(4)$ \\
\hline$C(4)-C(3)-C(2)$ & $120.6(4)$ & $C(27)-C(26)-C(25)$ & $116.3(4)$ \\
\hline$C(3)-C(4)-C(5)$ & 119.7(3) & $\mathrm{C}(27)-\mathrm{C}(26)-\mathrm{P}(2)$ & $118.4(3)$ \\
\hline$C(4)-C(5)-C(6)$ & $120.0(4)$ & $\mathrm{C}(25)-\mathrm{C}(26)-\mathrm{P}(2)$ & $125.3(3)$ \\
\hline$C(5)-C(6)-C(1)$ & $120.8(4)$ & $C(22)-C(27)-C(26)$ & $125.4(3)$ \\
\hline$C(8)-C(7)-C(12)$ & $118.9(4)$ & $\mathrm{C}(22)-\mathrm{C}(27)-\mathrm{O}(1)$ & 119.1(3) \\
\hline $\mathrm{C}(8)-\mathrm{C}(7)-\mathrm{P}(1)$ & $123.5(3)$ & $\mathrm{C}(26)-\mathrm{C}(27)-\mathrm{O}(1)$ & $115.6(3)$ \\
\hline$C(12)-C(7)-P(1)$ & $117.5(3)$ & $C(33)-C(28)-C(29)$ & $118.9(3)$ \\
\hline $\mathrm{C}(9)-\mathrm{C}(8)-\mathrm{C}(7)$ & $120.7(4)$ & $\mathrm{C}(33)-\mathrm{C}(28)-\mathrm{P}(2)$ & 119.1(3) \\
\hline$C(10)-C(9)-C(8)$ & $120.5(5)$ & $\mathrm{C}(29)-\mathrm{C}(28)-\mathrm{P}(2)$ & $122.1(3)$ \\
\hline
\end{tabular}




$\begin{array}{lr}\mathrm{C}(30)-\mathrm{C}(29)-\mathrm{C}(28) & 120.1(4) \\ \mathrm{C}(31)-\mathrm{C}(30)-\mathrm{C}(29) & 120.5(4) \\ \mathrm{C}(30)-\mathrm{C}(31)-\mathrm{C}(32) & 119.9(4) \\ \mathrm{C}(33)-\mathrm{C}(32)-\mathrm{C}(31) & 120.0(4) \\ \mathrm{C}(32)-\mathrm{C}(33)-\mathrm{C}(28) & 120.6(4) \\ \mathrm{C}(39)-\mathrm{C}(34)-\mathrm{C}(35) & 119.1(3) \\ \mathrm{C}(39)-\mathrm{C}(34)-\mathrm{P}(2) & 124.7(3) \\ \mathrm{C}(35)-\mathrm{C}(34)-\mathrm{P}(2) & 116.2(3) \\ \mathrm{C}(36)-\mathrm{C}(35)-\mathrm{C}(34) & 119.8(4) \\ \mathrm{C}(37)-\mathrm{C}(36)-\mathrm{C}(35) & 120.8(4) \\ \mathrm{C}(36)-\mathrm{C}(37)-\mathrm{C}(38) & 120.0(4) \\ \mathrm{C}(39)-\mathrm{C}(38)-\mathrm{C}(37) & 119.6(4) \\ \mathrm{C}(34)-\mathrm{C}(39)-\mathrm{C}(38) & 120.6(3) \\ \mathrm{C}(41)-\mathrm{C}(40)-\mathrm{Pd}(1) & 70.4(2) \\ \mathrm{C}(40)-\mathrm{C}(41)-\mathrm{C}(42) & 117.7(4) \\ \mathrm{C}(40)-\mathrm{C}(41)-\mathrm{Pd}(1) & 71.8(2) \\ \mathrm{C}(42)-\mathrm{C}(41)-\mathrm{Pd}(1) & 71.9(3) \\ \mathrm{C}(41)-\mathrm{C}(42)-\mathrm{Pd}(1) & 70.4(3) \\ \mathrm{C}(48)-\mathrm{C}(43)-\mathrm{C}(44) & 121.5(8) \\ \mathrm{C}(45)-\mathrm{C}(44)-\mathrm{C}(43) & 114.5(9) \\ \mathrm{C}(44)-\mathrm{C}(45)-\mathrm{C}(46) & 127.5(9) \\ \mathrm{C}(47)-\mathrm{C}(46)-\mathrm{C}(45) & 117.5(7) \\ \mathrm{C}(48)-\mathrm{C}(47)-\mathrm{C}(46) & 116.9(8) \\ \mathrm{C}(47)-\mathrm{C}(48)-\mathrm{C}(43) & 121.9(9) \\ & \end{array}$


Table S4. Anisotropic displacement parameters $\left(\AA^{2} \times 10^{3}\right)$ for 1 . The anisotropic displacement factor exponent takes the form: $-2 \pi^{2}\left[h^{2} a^{* 2} U^{11}+\ldots+2 h k a^{*} b^{*} U^{12}\right]$

\begin{tabular}{|c|c|c|c|c|c|c|}
\hline & $\mathrm{U}^{11}$ & $\mathrm{U}^{22}$ & $\mathrm{U}^{33}$ & $\mathrm{U}^{23}$ & $\mathrm{U}^{13}$ & $\mathrm{U}^{12}$ \\
\hline $\operatorname{Pd}(1)$ & $18(1)$ & $25(1)$ & $25(1)$ & $1(1)$ & $3(1)$ & $0(1)$ \\
\hline $\mathrm{Cl}(1)$ & $73(1)$ & $35(1)$ & $27(1)$ & $-3(1)$ & $8(1)$ & $9(1)$ \\
\hline $\mathrm{P}(1)$ & $23(1)$ & $23(1)$ & $20(1)$ & $0(1)$ & $4(1)$ & $1(1)$ \\
\hline $\mathrm{P}(2)$ & $24(1)$ & $22(1)$ & $22(1)$ & $0(1)$ & $2(1)$ & $1(1)$ \\
\hline $\mathrm{O}(1)$ & $23(2)$ & $25(1)$ & $23(1)$ & $0(1)$ & $-1(1)$ & $-1(1)$ \\
\hline $\mathrm{C}(1)$ & $32(2)$ & $15(2)$ & $22(2)$ & $1(2)$ & $5(2)$ & $-1(2)$ \\
\hline $\mathrm{C}(2)$ & $32(2)$ & $31(2)$ & $25(2)$ & $-3(2)$ & $4(2)$ & $-7(2)$ \\
\hline $\mathrm{C}(3)$ & $41(3)$ & $45(3)$ & $29(2)$ & $-2(2)$ & $13(2)$ & $8(2)$ \\
\hline$C(4)$ & $54(3)$ & $28(2)$ & $22(2)$ & $3(2)$ & $8(2)$ & $6(2)$ \\
\hline $\mathrm{C}(5)$ & $46(3)$ & $25(2)$ & $23(2)$ & $4(2)$ & $-6(2)$ & $-2(2)$ \\
\hline$C(6)$ & $34(3)$ & $30(2)$ & $27(2)$ & $2(2)$ & $5(2)$ & $2(2)$ \\
\hline$C(7)$ & $31(2)$ & $23(2)$ & $29(2)$ & $2(2)$ & $-1(2)$ & $4(2)$ \\
\hline $\mathrm{C}(8)$ & $69(4)$ & $30(3)$ & $45(3)$ & $-1(2)$ & $-9(2)$ & $18(3)$ \\
\hline $\mathrm{C}(9)$ & $99(5)$ & $50(3)$ & $69(4)$ & $-10(3)$ & $-41(4)$ & $29(3)$ \\
\hline$C(10)$ & $65(4)$ & $41(3)$ & $122(5)$ & $-9(3)$ & $-31(4)$ & $26(3)$ \\
\hline$C(11)$ & $41(3)$ & $35(3)$ & $92(4)$ & $-15(3)$ & $15(3)$ & $10(2)$ \\
\hline$C(12)$ & $31(3)$ & $27(2)$ & $49(3)$ & $-5(2)$ & $5(2)$ & $2(2)$ \\
\hline$C(13)$ & $28(2)$ & $23(2)$ & $24(2)$ & $-1(2)$ & $8(2)$ & $-4(2)$ \\
\hline$C(14)$ & $33(3)$ & $26(2)$ & $32(2)$ & $2(2)$ & $10(2)$ & $-1(2)$ \\
\hline$C(15)$ & $48(3)$ & $27(2)$ & $46(2)$ & $-5(2)$ & $18(2)$ & $-10(2)$ \\
\hline$C(16)$ & $37(3)$ & $40(3)$ & $35(2)$ & $-15(2)$ & $17(2)$ & $-20(2)$ \\
\hline$C(17)$ & $23(2)$ & $37(2)$ & $25(2)$ & $-6(2)$ & $4(2)$ & $-7(2)$ \\
\hline$C(18)$ & $24(2)$ & $31(2)$ & $21(2)$ & $-2(2)$ & $7(2)$ & $-5(2)$ \\
\hline$C(19)$ & $20(2)$ & $46(3)$ & $37(2)$ & $-8(2)$ & $3(2)$ & $-7(2)$ \\
\hline$C(20)$ & $30(3)$ & $65(3)$ & $44(3)$ & $-11(2)$ & $-5(2)$ & $-16(2)$ \\
\hline $\mathrm{C}(21)$ & $26(2)$ & $66(3)$ & $41(2)$ & $-13(2)$ & $6(2)$ & $3(2)$ \\
\hline $\mathrm{C}(22)$ & $24(2)$ & $36(2)$ & $27(2)$ & $-11(2)$ & $-2(2)$ & $0(2)$ \\
\hline$C(23)$ & $23(2)$ & $43(3)$ & $35(2)$ & $-12(2)$ & $-8(2)$ & $10(2)$ \\
\hline$C(24)$ & $34(3)$ & $39(3)$ & $28(2)$ & $-4(2)$ & $-9(2)$ & $12(2)$ \\
\hline$C(25)$ & $38(3)$ & $32(2)$ & $27(2)$ & $-3(2)$ & $2(2)$ & $12(2)$ \\
\hline
\end{tabular}




\begin{tabular}{lllllll}
$\mathrm{C}(26)$ & $23(2)$ & $26(2)$ & $26(2)$ & $-6(2)$ & $3(2)$ & $9(2)$ \\
$\mathrm{C}(27)$ & $22(2)$ & $29(2)$ & $20(2)$ & $-5(2)$ & $2(2)$ & $3(2)$ \\
$\mathrm{C}(28)$ & $30(2)$ & $22(2)$ & $23(2)$ & $1(2)$ & $3(2)$ & $2(2)$ \\
$\mathrm{C}(29)$ & $35(3)$ & $42(3)$ & $31(2)$ & $-5(2)$ & $1(2)$ & $7(2)$ \\
$\mathrm{C}(30)$ & $32(3)$ & $50(3)$ & $45(3)$ & $-9(2)$ & $9(2)$ & $16(2)$ \\
$\mathrm{C}(31)$ & $55(3)$ & $35(3)$ & $34(2)$ & $-1(2)$ & $16(2)$ & $8(2)$ \\
$\mathrm{C}(32)$ & $62(3)$ & $37(3)$ & $23(2)$ & $-2(2)$ & $-6(2)$ & $9(2)$ \\
$\mathrm{C}(33)$ & $36(3)$ & $29(2)$ & $31(2)$ & $-2(2)$ & $1(2)$ & $7(2)$ \\
$\mathrm{C}(34)$ & $25(2)$ & $31(2)$ & $25(2)$ & $1(2)$ & $0(2)$ & $0(2)$ \\
$\mathrm{C}(35)$ & $46(3)$ & $24(2)$ & $28(2)$ & $-1(2)$ & $5(2)$ & $1(2)$ \\
$\mathrm{C}(36)$ & $37(3)$ & $41(3)$ & $32(2)$ & $3(2)$ & $6(2)$ & $4(2)$ \\
$\mathrm{C}(37)$ & $39(3)$ & $38(3)$ & $38(2)$ & $15(2)$ & $1(2)$ & $-5(2)$ \\
$\mathrm{C}(38)$ & $47(3)$ & $27(2)$ & $51(3)$ & $1(2)$ & $8(2)$ & $-1(2)$ \\
$\mathrm{C}(39)$ & $33(3)$ & $27(2)$ & $35(2)$ & $-1(2)$ & $8(2)$ & $5(2)$ \\
$\mathrm{C}(40)$ & $22(2)$ & $43(3)$ & $72(3)$ & $17(2)$ & $7(2)$ & $-14(2)$ \\
$\mathrm{C}(41)$ & $22(3)$ & $44(3)$ & $53(3)$ & $1(2)$ & $-7(2)$ & $-11(2)$ \\
$\mathrm{C}(42)$ & $14(2)$ & $51(3)$ & $50(3)$ & $6(2)$ & $3(2)$ & $-2(2)$ \\
$\mathrm{C}(43)$ & $149(8)$ & $154(9)$ & $84(5)$ & $-55(6)$ & $-51(5)$ & $88(7)$ \\
$\mathrm{C}(44)$ & $130(7)$ & $105(7)$ & $94(5)$ & $-27(5)$ & $-18(5)$ & $55(6)$ \\
$\mathrm{C}(45)$ & $108(6)$ & $119(7)$ & $78(5)$ & $-8(5)$ & $-21(4)$ & $-21(6)$ \\
$\mathrm{C}(46)$ & $57(4)$ & $124(7)$ & $122(6)$ & $-55(6)$ & $-43(4)$ & $26(5)$ \\
$\mathrm{C}(47)$ & $72(5)$ & $110(7)$ & $109(6)$ & $-18(5)$ & $-27(4)$ & $-11(5)$ \\
$\mathrm{C}(48)$ & $138(8)$ & $112(7)$ & $98(6)$ & $-30(6)$ & $-55(6)$ & $-9(6)$ \\
& & & & & & \\
\hline
\end{tabular}


Table S5. Hydrogen coordinates ( $\left.\times 10^{4}\right)$ and isotropic displacement parameters $\left(\AA^{2} \times 10^{3}\right)$ for 1.

\begin{tabular}{|c|c|c|c|c|}
\hline & $\mathrm{x}$ & $\mathrm{y}$ & $\mathrm{z}$ & $\mathrm{U}(\mathrm{eq})$ \\
\hline $\mathrm{H}(2 \mathrm{~A})$ & 5491 & 1563 & 2220 & 35 \\
\hline $\mathrm{H}(3 \mathrm{~A})$ & 5088 & 2262 & 3142 & 45 \\
\hline $\mathrm{H}(4 \mathrm{~A})$ & 6530 & 2834 & 3783 & 41 \\
\hline $\mathrm{H}(5 \mathrm{~A})$ & 8391 & 2774 & 3477 & 38 \\
\hline $\mathrm{H}(6 \mathrm{~A})$ & 8790 & 2159 & 2522 & 36 \\
\hline $\mathrm{H}(8 \mathrm{~A})$ & 8493 & 504 & 2634 & 58 \\
\hline $\mathrm{H}(9 \mathrm{~A})$ & 9991 & -405 & 2820 & 89 \\
\hline $\mathrm{H}(10 \mathrm{~A})$ & 10973 & -917 & 1998 & 93 \\
\hline $\mathrm{H}(11 \mathrm{~A})$ & 10418 & -555 & 959 & 67 \\
\hline $\mathrm{H}(12 \mathrm{~A})$ & 8922 & 385 & 774 & 43 \\
\hline $\mathrm{H}(14 \mathrm{~A})$ & 6870 & -446 & 1505 & 36 \\
\hline $\mathrm{H}(15 \mathrm{~A})$ & 5259 & -1157 & 1139 & 48 \\
\hline $\mathrm{H}(16 \mathrm{~A})$ & 3705 & -452 & 692 & 44 \\
\hline $\mathrm{H}(20 \mathrm{~A})$ & 2825 & 552 & -202 & 70 \\
\hline $\mathrm{H}(20 \mathrm{~B})$ & 1890 & 1191 & 24 & 70 \\
\hline $\mathrm{H}(20 \mathrm{C})$ & 2173 & 356 & 418 & 70 \\
\hline $\mathrm{H}(21 \mathrm{~A})$ & 3413 & 1976 & 1390 & 66 \\
\hline $\mathrm{H}(21 \mathrm{~B})$ & 2521 & 1223 & 1385 & 66 \\
\hline $\mathrm{H}(21 \mathrm{C})$ & 2242 & 2055 & 986 & 66 \\
\hline $\mathrm{H}(23 \mathrm{~A})$ & 2805 & 2110 & -562 & 41 \\
\hline $\mathrm{H}(24 \mathrm{~A})$ & 3697 & 3230 & -1022 & 41 \\
\hline $\mathrm{H}(25 \mathrm{~A})$ & 5366 & 3783 & -574 & 39 \\
\hline $\mathrm{H}(29 \mathrm{~A})$ & 4816 & 4404 & 808 & 43 \\
\hline $\mathrm{H}(30 \mathrm{~A})$ & 4191 & 5146 & 1662 & 51 \\
\hline $\mathrm{H}(31 \mathrm{~A})$ & 5211 & 5120 & 2617 & 49 \\
\hline $\mathrm{H}(32 \mathrm{~A})$ & 6896 & 4368 & 2722 & 49 \\
\hline $\mathrm{H}(33 \mathrm{~A})$ & 7546 & 3642 & 1869 & 38 \\
\hline $\mathrm{H}(35 \mathrm{~A})$ & 7821 & 3278 & -551 & 39 \\
\hline $\mathrm{H}(36 \mathrm{~A})$ & 8439 & 4230 & -1288 & 44 \\
\hline
\end{tabular}




\begin{tabular}{lrrrr}
$\mathrm{H}(37 \mathrm{~A})$ & 8259 & 5656 & -1134 & 46 \\
$\mathrm{H}(38 \mathrm{~A})$ & 7476 & 6162 & -222 & 50 \\
$\mathrm{H}(39 \mathrm{~A})$ & 6836 & 5216 & 517 & 38 \\
$\mathrm{H}(40 \mathrm{~A})$ & 9932 & 3239 & 265 & 54 \\
$\mathrm{H}(40 \mathrm{~B})$ & 9312 & 3962 & 684 & 54 \\
$\mathrm{H}(41 \mathrm{~A})$ & 10013 & 3271 & 1603 & 48 \\
$\mathrm{H}(42 \mathrm{~A})$ & 10107 & 1791 & 1595 & 46 \\
$\mathrm{H}(42 \mathrm{~B})$ & 10445 & 1838 & 853 & 46 \\
$\mathrm{H}(43 \mathrm{~A})$ & 12432 & 1079 & 2628 & 157 \\
$\mathrm{H}(44 \mathrm{~A})$ & 12428 & 2547 & 2477 & 133 \\
$\mathrm{H}(45 \mathrm{~A})$ & 11483 & 3297 & 3137 & 124 \\
$\mathrm{H}(46 \mathrm{~A})$ & 10468 & 2756 & 3937 & 123 \\
$\mathrm{H}(47 \mathrm{~A})$ & 10535 & 1299 & 4120 & 118 \\
$\mathrm{H}(48 \mathrm{~A})$ & 11613 & 498 & 3475 & 142 \\
& & & & \\
\hline
\end{tabular}




\section{Experimental Procedure for the X-Ray Diffraction of 6}

\section{$\underline{\text { Data Collection }}$}

An orange plate crystal of $\mathrm{C}_{47} \mathrm{H}_{39} \mathrm{~F}_{3} \mathrm{O}_{4} \mathrm{P}_{2} \mathrm{PdS}$ having approximate dimensions of $0.20 \mathrm{x}$ $0.10 \times 0.10 \mathrm{~mm}^{3}$ was mounted with epoxy cement on the tip of a fine glass fiber. All measurements were made on a Nonius KappaCCD diffractometer with graphite monochromated Mo-K $\alpha$ radiation.

Cell constants and an orientation matrix for data collection corresponded to a primitive monoclinic cell with dimensions:

$$
\begin{array}{ll}
\mathrm{a}=18.235(4) \AA & \alpha=90 \mathrm{o} \\
\mathrm{b}=18.040(4) \AA & \beta=99.73(3) \mathrm{o} \\
\mathrm{c}=25.472(5) \AA & \gamma=90^{\circ} \\
\mathrm{V}=8259(3) \AA^{3} &
\end{array}
$$

For $\mathrm{Z}=4$ and F.W. $=925.18$, the calculated density is $1.488 \mathrm{~g} / \mathrm{cm}^{3}$. Based on a statistical analysis of intensity distribution, and the successful solution and refinement of the structure, the space group was determined to be $P 2{ }_{1} / c$ (\#14).

The data were collected at a temperature of $173(2) \mathrm{K}$ to a maximum $2 \theta$ value of $50.50^{\circ}$. Four omega scans consisting of 76, 69, 76, and 41 data frames, respectively, were collected with a frame width of $1.10^{\circ}$ and a detector-to-crystal distance, Dx, of $45.0 \mathrm{~mm}$. Each frame was exposed twice (for the purpose of de-zingering) for a total of $88 \mathrm{~s}$. The data frames were processed and scaled using the DENZO software package. ${ }^{1}$

\section{Data Reduction}

A total of 26119 reflections were collected of which 15309 were unique and observed $\left(\mathrm{R}_{\text {int }}=0.0616\right)$. The linear absorption coefficient, $\mu$, for Mo-K $\alpha$ radiation is $6.36 \mathrm{~cm}^{-1}$, and no absorption correction was applied. The data were corrected for Lorentz and polarization effects.

\section{$\underline{\text { Structure Solution and Refinement }}$}

The structure was solved by direct methods and expanded using Fourier techniques ${ }^{2}$. The non-hydrogen atoms were refined anisotropically, and hydrogen atoms were treated as idealized contributions. The final cycle of full-matrix least-squares refinement ${ }^{3}$ on $\mathrm{F}$ was based on 15309 observed reflections $(\mathrm{I}>2.00 \sigma(\mathrm{I}))$ and 1045 variable parameters and converged with unweighted and weighted agreement factors of:

$$
\begin{gathered}
\mathrm{R}=\Sigma\|\mathrm{Fo}|-| \mathrm{Fc}\| / \Sigma|\mathrm{Fo}|=0.0644 \\
\mathrm{R}_{\mathrm{W}}=\left\{\Sigma\left[\mathrm{w}\left(\mathrm{F}_{\mathrm{o}}^{2}-\mathrm{F}_{\mathrm{c}}{ }^{2}\right)^{2}\right] / \Sigma\left[\mathrm{w}\left(\mathrm{F}_{\mathrm{o}}\right)^{2}\right]\right\}^{1 / 2}=0.1668
\end{gathered}
$$


The maximum and minimum peaks on the final difference Fourier map corresponded to 1.498 and $-1.022 \mathrm{e}^{-/} \AA^{3}$ respectively.

\section{REFERENCES}

(1) Z. Otwinowski and W. Minor, "Processing of X-Ray Diffraction Data Collected in Oscillation Mode," Methods in Enzymology, vol. 276: Macromolecular Crystallography, part A, 307-326, 1997, C.W. Carter, Jr. \& R.M. Sweet, Eds., Academic Press.

(2) Acta Cryst. A46 (1990) 467-473

(3) Least Squares function minimized:

$$
\Sigma w\left(\mathrm{~F}_{\mathrm{o}}^{2}-\mathrm{F}_{\mathrm{c}}^{2}\right)^{2}
$$




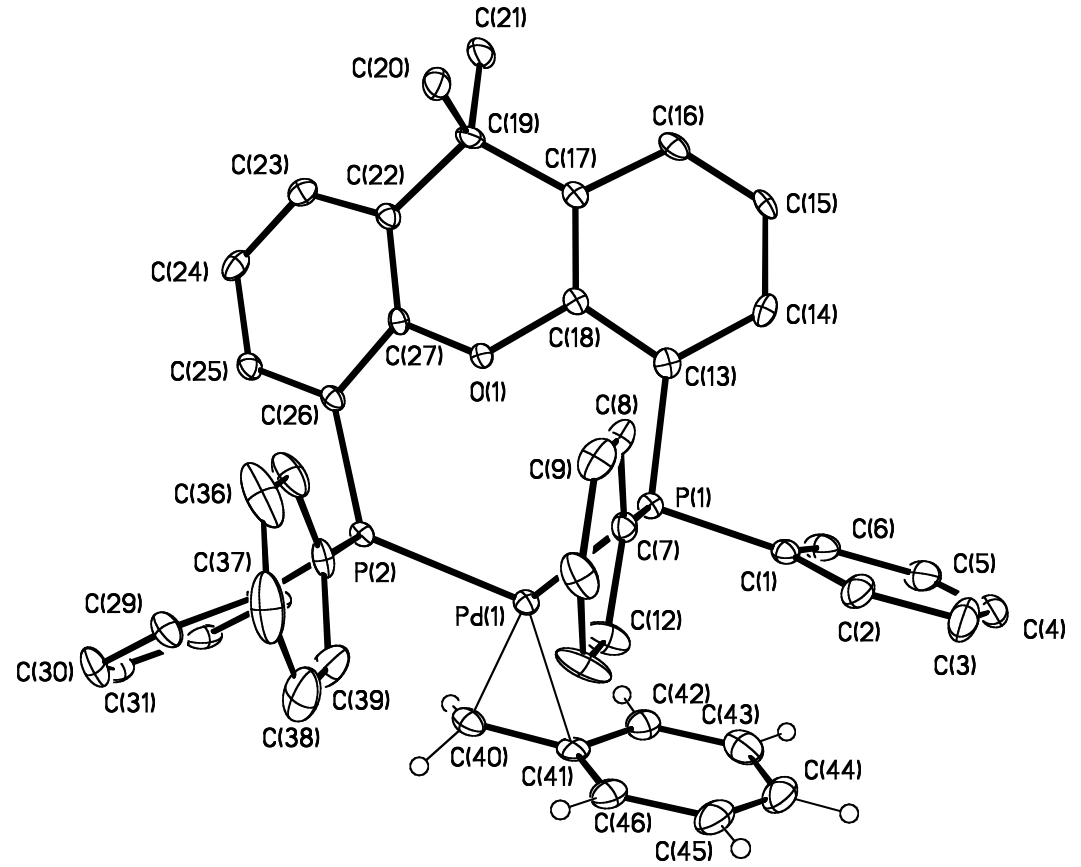

Figure S5. ORTEP diagram of $6\left(1^{\text {st }}\right.$ independent molecule $)$

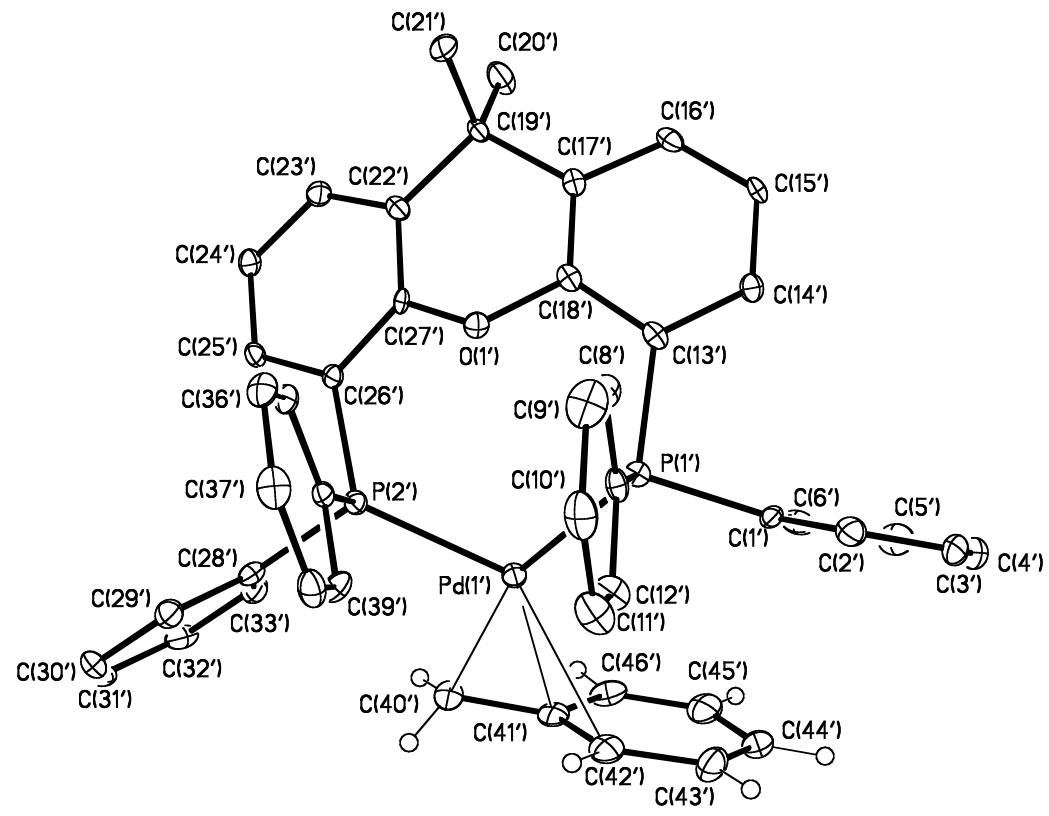

Figure S6. ORTEP diagram of 6 ( $2^{\text {st }}$ independent molecule) 
Table S6. Crystal data and structure refinement for 6.

Empirical formula

Formula weight

Temperature

Wavelength

Crystal system

Space group

Unit cell dimensions

Volume

Z

Density (calculated)

Absorption coefficient

$\mathrm{F}(000)$

Crystal size

Theta range for data collection

Index ranges

Reflections collected

Independent reflections

Completeness to theta $=25.50^{\circ}$

Absorption correction

Max. and min. transmission

Refinement method

Data / restraints / parameters

Goodness-of-fit on $\mathrm{F}^{2}$

Final $\mathrm{R}$ indices $[\mathrm{I}>2 \operatorname{sigma}(\mathrm{I})]$

$\mathrm{R}$ indices (all data)

Largest diff. peak and hole
$\mathrm{C}_{47} \mathrm{H}_{39} \mathrm{~F}_{3} \mathrm{O}_{4} \mathrm{P}_{2} \mathrm{Pd} \mathrm{S}$

925.18

173(2) K

$0.71073 \AA$

Monoclinic

P2(1)/c

$\mathrm{a}=18.235(4) \AA$ $\alpha=90^{\circ}$.

$\mathrm{b}=18.040(4) \AA$ $\beta=99.73(3)^{\circ}$.

$\mathrm{c}=25.472(5) \AA$ $\gamma=90^{\circ}$.

8259(3) $\AA^{3}$

8

$1.488 \mathrm{~g} / \mathrm{cm}^{3}$

$6.36 \mathrm{~cm}^{-1}$

3776

$0.20 \times 0.10 \times 0.10 \mathrm{~mm}^{3}$

1.70 to $25.50^{\circ}$.

$-22<=\mathrm{h}<=22,-21<=\mathrm{k}<=18,-30<=\mathrm{l}<=30$

26119

$15309[\mathrm{R}(\mathrm{int})=0.0616]$

$99.7 \%$

None

0.9392 and 0.8834

Full-matrix least-squares on $\mathrm{F}^{2}$

15309 / 0 / 1045

1.069

$\mathrm{R} 1=0.0644, \mathrm{wR} 2=0.1668$

$\mathrm{R} 1=0.1137, \mathrm{wR} 2=0.1889$

1.498 and -1.022 e. $\AA^{-3}$ 
Table S7. Atomic coordinates ( $\times 10^{4}$ ) and equivalent isotropic displacement parameters $\left(\AA^{2} \times 1^{3}\right)$ for $6 . U\left(\right.$ eq) is defined as one third of the trace of the orthogonalized $U^{i j}$ tensor.

\begin{tabular}{|c|c|c|c|c|}
\hline & $\mathrm{X}$ & $\mathrm{y}$ & $\mathrm{z}$ & $\mathrm{U}(\mathrm{eq})$ \\
\hline $\operatorname{Pd}(1)$ & $2197(1)$ & $5756(1)$ & $3731(1)$ & $18(1)$ \\
\hline $\mathrm{P}(1)$ & $3363(1)$ & $5500(1)$ & $4296(1)$ & $17(1)$ \\
\hline $\mathrm{P}(2)$ & $1258(1)$ & $5561(1)$ & $4219(1)$ & $16(1)$ \\
\hline $\mathrm{O}(1)$ & $2475(2)$ & $5722(2)$ & $5094(1)$ & $17(1)$ \\
\hline $\mathrm{C}(1)$ & $4150(3)$ & $5649(3)$ & $3964(2)$ & $20(1)$ \\
\hline$C(2)$ & $4672(3)$ & $5098(4)$ & $3898(2)$ & $32(2)$ \\
\hline$C(3)$ & $5287(4)$ & $5257(5)$ & $3668(3)$ & $44(2)$ \\
\hline$C(4)$ & $5385(4)$ & $5965(5)$ & $3484(3)$ & $50(2)$ \\
\hline$C(5)$ & $4879(4)$ & $6509(4)$ & $3532(2)$ & $39(2)$ \\
\hline$C(6)$ & $4261(4)$ & $6352(4)$ & $3770(2)$ & $30(2)$ \\
\hline$C(7)$ & $3410(3)$ & $4521(3)$ & $4470(2)$ & $20(1)$ \\
\hline $\mathrm{C}(8)$ & $3633(3)$ & $4266(3)$ & $4986(2)$ & $25(1)$ \\
\hline $\mathrm{C}(9)$ & $3658(3)$ & $3504(3)$ & $5092(2)$ & $29(2)$ \\
\hline$C(10)$ & $3468(3)$ & $3002(4)$ & $4686(3)$ & $32(2)$ \\
\hline$C(11)$ & $3231(4)$ & $3251(4)$ & $4176(3)$ & $40(2)$ \\
\hline$C(12)$ & $3206(4)$ & $4001(4)$ & $4063(3)$ & $36(2)$ \\
\hline$C(13)$ & $3669(3)$ & $5975(3)$ & $4925(2)$ & $17(1)$ \\
\hline$C(14)$ & 4381(3) & $6275(3)$ & $5095(2)$ & 21(1) \\
\hline$C(15)$ & $4525(3)$ & $6652(3)$ & $5569(2)$ & $19(1)$ \\
\hline$C(16)$ & 3999(3) & $6752(3)$ & $5893(2)$ & $23(1)$ \\
\hline$C(17)$ & $3279(3)$ & $6458(3)$ & $5741(2)$ & $17(1)$ \\
\hline$C(18)$ & $3148(3)$ & $6075(3)$ & $5262(2)$ & $15(1)$ \\
\hline$C(19)$ & $2663(3)$ & $6519(3)$ & $6075(2)$ & $21(1)$ \\
\hline$C(20)$ & $2744(3)$ & $5864(4)$ & $6471(2)$ & $34(2)$ \\
\hline$C(21)$ & $2728(3)$ & $7250(4)$ & $6390(3)$ & $40(2)$ \\
\hline $\mathrm{C}(22)$ & 1914(3) & $6465(3)$ & $5708(2)$ & $19(1)$ \\
\hline$C(23)$ & $1252(3)$ & $6768(3)$ & $5819(2)$ & $23(1)$ \\
\hline$C(24)$ & $597(3)$ & $6697(3)$ & $5467(2)$ & $25(2)$ \\
\hline$C(25)$ & $573(3)$ & $6340(3)$ & $4983(2)$ & $21(1)$ \\
\hline$C(26)$ & $1219(3)$ & $6027(3)$ & $4851(2)$ & $17(1)$ \\
\hline$C(27)$ & 1861(3) & $6086(3)$ & $5226(2)$ & $16(1)$ \\
\hline
\end{tabular}




\begin{tabular}{|c|c|c|c|c|}
\hline$C(28)$ & $304(3)$ & $5694(3)$ & $3873(2)$ & $21(1)$ \\
\hline C(29) & $-241(3)$ & $5159(4)$ & $3869(2)$ & $31(2)$ \\
\hline$C(30)$ & $-963(4)$ & $5325(5)$ & $3626(3)$ & $47(2)$ \\
\hline$C(31)$ & $-1152(4)$ & $5999(5)$ & $3394(3)$ & $41(2)$ \\
\hline$C(32)$ & $-614(4)$ & $6523(4)$ & $3392(2)$ & $32(2)$ \\
\hline$C(33)$ & $110(3)$ & $6388(3)$ & $3628(2)$ & $24(1)$ \\
\hline$C(34)$ & $1308(3)$ & $4570(3)$ & $4386(2)$ & $24(1)$ \\
\hline$C(35)$ & $1389(3)$ & 4313(4) & 4897(3) & $38(2)$ \\
\hline$C(36)$ & $1459(4)$ & $3564(5)$ & $5006(4)$ & $54(2)$ \\
\hline$C(37)$ & $1439(4)$ & $3065(4)$ & $4600(4)$ & $55(2)$ \\
\hline $\mathrm{C}(38)$ & $1347(4)$ & $3320(4)$ & $4077(4)$ & $55(2)$ \\
\hline$C(39)$ & $1283(4)$ & $4069(4)$ & $3967(3)$ & $43(2)$ \\
\hline$C(40)$ & $1435(3)$ & $5873(4)$ & $3027(2)$ & $28(2)$ \\
\hline $\mathrm{C}(41)$ & $2148(3)$ & $5848(4)$ & $2850(2)$ & $26(2)$ \\
\hline$C(42)$ & $2524(4)$ & $6501(4)$ & $2750(2)$ & $37(2)$ \\
\hline$C(43)$ & $3227(4)$ & $6457(5)$ & $2603(3)$ & $46(2)$ \\
\hline$C(44)$ & $3553(4)$ & $5771(5)$ & $2563(3)$ & $48(2)$ \\
\hline$C(45)$ & $3215(4)$ & $5122(4)$ & $2688(2)$ & $42(2)$ \\
\hline$C(46)$ & $2514(4)$ & $5169(4)$ & $2827(2)$ & $35(2)$ \\
\hline $\mathrm{S}(1)$ & $5145(1)$ & $6881(1)$ & $7396(1)$ & $29(1)$ \\
\hline $\mathrm{O}(2)$ & $5480(3)$ & $6540(3)$ & $6974(2)$ & $45(1)$ \\
\hline $\mathrm{O}(3)$ & $5653(2)$ & $7255(2)$ & $7808(2)$ & $30(1)$ \\
\hline $\mathrm{O}(4)$ & $4455(2)$ & $7274(3)$ & $7220(2)$ & $36(1)$ \\
\hline$C(47)$ & $4859(4)$ & $6080(4)$ & $7735(3)$ & $41(2)$ \\
\hline $\mathrm{F}(1)$ & $4372(2)$ & $5656(2)$ & $7412(2)$ & $64(1)$ \\
\hline $\mathrm{F}(2)$ & $5441(2)$ & $5643(2)$ & $7918(2)$ & $58(1)$ \\
\hline $\mathrm{F}(3)$ & $4538(2)$ & $6297(3)$ & $8153(2)$ & $56(1)$ \\
\hline $\operatorname{Pd}\left(1^{\prime}\right)$ & $2096(1)$ & $802(1)$ & $3779(1)$ & $18(1)$ \\
\hline $\mathrm{P}\left(1^{\prime}\right)$ & $3282(1)$ & $503(1)$ & $4305(1)$ & $17(1)$ \\
\hline $\mathrm{P}\left(2^{\prime}\right)$ & $1195(1)$ & $549(1)$ & 4291(1) & $17(1)$ \\
\hline $\mathrm{O}\left(1^{\prime}\right)$ & $2416(2)$ & $776(2)$ & $5127(1)$ & $17(1)$ \\
\hline$C\left(1^{\prime}\right)$ & $4071(3)$ & $726(3)$ & $3979(2)$ & $18(1)$ \\
\hline $\mathrm{C}\left(2^{\prime}\right)$ & $4664(3)$ & $238(4)$ & $3950(2)$ & $26(1)$ \\
\hline$C\left(3^{\prime}\right)$ & $5288(3)$ & $503(4)$ & $3760(2)$ & $33(2)$ \\
\hline$C\left(4^{\prime}\right)$ & $5321(3)$ & $1232(4)$ & $3598(2)$ & $32(2)$ \\
\hline$C\left(5^{\prime}\right)$ & $4741(4)$ & $1705(4)$ & $3624(2)$ & $35(2)$ \\
\hline
\end{tabular}




\begin{tabular}{|c|c|c|c|c|}
\hline$C\left(6^{\prime}\right)$ & $4115(3)$ & $1455(4)$ & $3810(2)$ & $27(1)$ \\
\hline$C\left(7^{\prime}\right)$ & $3352(3)$ & $-493(3)$ & $4416(2)$ & $19(1)$ \\
\hline $\mathrm{C}\left(8^{\prime}\right)$ & $3537(3)$ & $-807(4)$ & $4918(2)$ & $28(2)$ \\
\hline$C\left(9^{\prime}\right)$ & $3584(4)$ & $-1570(4)$ & $4975(3)$ & $36(2)$ \\
\hline$C\left(10^{\prime}\right)$ & $3438(3)$ & $-2021(4)$ & $4536(3)$ & $32(2)$ \\
\hline$C\left(11^{\prime}\right)$ & $3251(3)$ & $-1722(4)$ & $4026(3)$ & $34(2)$ \\
\hline$C\left(12^{\prime}\right)$ & $3204(3)$ & $-960(4)$ & $3970(2)$ & $29(2)$ \\
\hline$C\left(13^{\prime}\right)$ & $3617(3)$ & 933(3) & $4954(2)$ & $16(1)$ \\
\hline$C\left(14^{\prime}\right)$ & $4345(3)$ & $1190(3)$ & $5117(2)$ & $20(1)$ \\
\hline$C\left(15^{\prime}\right)$ & $4531(3)$ & $1581(3)$ & $5584(2)$ & $22(1)$ \\
\hline$C\left(16^{\prime}\right)$ & $3994(3)$ & $1758(3)$ & $5889(2)$ & $20(1)$ \\
\hline$C\left(17^{\prime}\right)$ & $3260(3)$ & $1513(3)$ & $5745(2)$ & $16(1)$ \\
\hline$C\left(18^{\prime}\right)$ & $3105(3)$ & $1089(3)$ & $5286(2)$ & $17(1)$ \\
\hline$C\left(19^{\prime}\right)$ & $2644(3)$ & $1690(3)$ & $6069(2)$ & $20(1)$ \\
\hline$C\left(20^{\prime}\right)$ & $2746(3)$ & $2496(4)$ & $6292(3)$ & $33(2)$ \\
\hline$C\left(21^{\prime}\right)$ & $2721(3)$ & $1142(4)$ & $6546(2)$ & $36(2)$ \\
\hline$C\left(22^{\prime}\right)$ & $1890(3)$ & 1601(3) & $5715(2)$ & $18(1)$ \\
\hline$C\left(23^{\prime}\right)$ & $1237(3)$ & $1917(3)$ & $5821(2)$ & $23(1)$ \\
\hline$C\left(24^{\prime}\right)$ & $566(3)$ & $1817(3)$ & $5498(2)$ & $20(1)$ \\
\hline$C\left(25^{\prime}\right)$ & $520(3)$ & 1398(3) & $5030(2)$ & $19(1)$ \\
\hline$C\left(26^{\prime}\right)$ & $1158(3)$ & $1076(3)$ & $4905(2)$ & $15(1)$ \\
\hline$C\left(27^{\prime}\right)$ & $1822(3)$ & $1165(3)$ & $5256(2)$ & $14(1)$ \\
\hline$C\left(28^{\prime}\right)$ & $225(3)$ & $610(3)$ & $3950(2)$ & $19(1)$ \\
\hline$C\left(29^{\prime}\right)$ & $-239(3)$ & $-4(4)$ & $3884(2)$ & $26(1)$ \\
\hline$C\left(30^{\prime}\right)$ & $-967(3)$ & $68(4)$ & $3632(2)$ & $33(2)$ \\
\hline$C\left(31^{\prime}\right)$ & $-1248(3)$ & $742(4)$ & $3436(2)$ & $33(2)$ \\
\hline$C\left(32^{\prime}\right)$ & $-786(3)$ & $1357(4)$ & $3493(2)$ & $28(2)$ \\
\hline$C\left(33^{\prime}\right)$ & $-59(3)$ & $1290(4)$ & $3746(2)$ & $27(2)$ \\
\hline$C\left(34^{\prime}\right)$ & $1320(3)$ & $-422(3)$ & $4500(2)$ & $18(1)$ \\
\hline$C\left(35^{\prime}\right)$ & $1360(3)$ & $-635(3)$ & $5025(2)$ & $21(1)$ \\
\hline$C\left(36^{\prime}\right)$ & $1485(3)$ & $-1376(3)$ & $5173(2)$ & $24(1)$ \\
\hline$C\left(37^{\prime}\right)$ & $1538(3)$ & $-1899(3)$ & $4780(2)$ & $26(2)$ \\
\hline$C\left(38^{\prime}\right)$ & $1483(3)$ & $-1680(3)$ & $4254(3)$ & $26(2)$ \\
\hline$C\left(39^{\prime}\right)$ & $1386(3)$ & $-945(3)$ & $4118(2)$ & $24(1)$ \\
\hline$C\left(40^{\prime}\right)$ & $1273(3)$ & 998(4) & $3113(2)$ & $30(2)$ \\
\hline$C\left(41^{\prime}\right)$ & $1953(3)$ & $1148(4)$ & $2923(2)$ & $26(1)$ \\
\hline
\end{tabular}




\begin{tabular}{lrrrr}
$\mathrm{C}\left(42^{\prime}\right)$ & $2424(3)$ & $559(4)$ & $2846(2)$ & $31(2)$ \\
$\mathrm{C}\left(43^{\prime}\right)$ & $3110(4)$ & $698(4)$ & $2687(3)$ & $39(2)$ \\
$\mathrm{C}\left(44^{\prime}\right)$ & $3327(4)$ & $1412(4)$ & $2610(2)$ & $35(2)$ \\
$\mathrm{C}\left(45^{\prime}\right)$ & $2881(4)$ & $2002(4)$ & $2700(2)$ & $40(2)$ \\
$\mathrm{C}\left(46^{\prime}\right)$ & $2212(4)$ & $1888(4)$ & $2858(2)$ & $33(2)$ \\
$\mathrm{S}\left(1^{\prime}\right)$ & $8951(1)$ & $1669(1)$ & $7394(1)$ & $60(1)$ \\
$\mathrm{O}\left(2^{\prime}\right)$ & $8860(5)$ & $1845(4)$ & $7920(2)$ & $113(3)$ \\
$\mathrm{O}\left(3^{\prime}\right)$ & $8665(4)$ & $953(3)$ & $7201(2)$ & $90(2)$ \\
$\mathrm{O}\left(4^{\prime}\right)$ & $8860(4)$ & $2274(3)$ & $7026(2)$ & $84(2)$ \\
$\mathrm{C}\left(47^{\prime}\right)$ & $9858(8)$ & $1690(12)$ & $7499(5)$ & $185(11)$ \\
$\mathrm{F}\left(1^{\prime}\right)$ & $10173(5)$ & $979(5)$ & $7759(4)$ & $199(5)$ \\
$\mathrm{F}\left(2^{\prime}\right)$ & $10155(4)$ & $1433(4)$ & $6991(3)$ & $128(2)$ \\
$\mathrm{F}\left(3^{\prime}\right)$ & $10309(4)$ & $2149(5)$ & $7692(3)$ & $141(3)$ \\
& & & & \\
\hline
\end{tabular}


Table S8. Bond lengths $[\AA]$ and angles $\left[{ }^{\circ}\right]$ for 6 .

\begin{tabular}{|c|c|c|c|}
\hline $\operatorname{Pd}(1)-C(40)$ & $2.085(6)$ & $\mathrm{C}(22)-\mathrm{C}(27)$ & $1.396(7)$ \\
\hline $\operatorname{Pd}(1)-C(41)$ & $2.238(5)$ & $\mathrm{C}(22)-\mathrm{C}(23)$ & $1.396(8)$ \\
\hline $\mathrm{Pd}(1)-\mathrm{P}(2)$ & $2.3063(16)$ & $\mathrm{C}(23)-\mathrm{C}(24)$ & $1.372(8)$ \\
\hline $\operatorname{Pd}(1)-\mathrm{P}(1)$ & $2.4031(17)$ & $C(24)-C(25)$ & $1.384(8)$ \\
\hline $\mathrm{P}(1)-\mathrm{C}(1)$ & $1.805(6)$ & $C(25)-C(26)$ & $1.398(7)$ \\
\hline$P(1)-C(13)$ & $1.818(6)$ & $C(26)-C(27)$ & $1.385(7)$ \\
\hline$P(1)-C(7)$ & $1.818(6)$ & $\mathrm{C}(28)-\mathrm{C}(29)$ & $1.384(8)$ \\
\hline $\mathrm{P}(2)-\mathrm{C}(28)$ & $1.827(6)$ & $\mathrm{C}(28)-\mathrm{C}(33)$ & $1.417(8)$ \\
\hline$P(2)-C(26)$ & $1.828(6)$ & $C(29)-C(30)$ & $1.389(9)$ \\
\hline$P(2)-C(34)$ & $1.836(6)$ & $C(30)-C(31)$ & $1.371(10)$ \\
\hline $\mathrm{O}(1)-\mathrm{C}(18)$ & $1.384(6)$ & $C(31)-C(32)$ & $1.364(9)$ \\
\hline $\mathrm{O}(1)-\mathrm{C}(27)$ & $1.387(6)$ & $C(32)-C(33)$ & $1.377(8)$ \\
\hline$C(1)-C(6)$ & $1.389(8)$ & $C(34)-C(35)$ & $1.366(8)$ \\
\hline$C(1)-C(2)$ & $1.405(8)$ & $\mathrm{C}(34)-\mathrm{C}(39)$ & $1.391(9)$ \\
\hline$C(2)-C(3)$ & $1.380(8)$ & $C(35)-C(36)$ & $1.381(9)$ \\
\hline$C(3)-C(4)$ & $1.383(11)$ & $C(36)-C(37)$ & $1.368(11)$ \\
\hline$C(4)-C(5)$ & $1.368(10)$ & $\mathrm{C}(37)-\mathrm{C}(38)$ & $1.393(11)$ \\
\hline$C(5)-C(6)$ & $1.395(8)$ & $\mathrm{C}(38)-\mathrm{C}(39)$ & $1.382(9)$ \\
\hline$C(7)-C(8)$ & $1.387(8)$ & $C(40)-C(41)$ & $1.447(8)$ \\
\hline$C(7)-C(12)$ & $1.401(8)$ & $C(41)-C(46)$ & $1.401(9)$ \\
\hline $\mathrm{C}(8)-\mathrm{C}(9)$ & $1.401(8)$ & $C(41)-C(42)$ & $1.407(9)$ \\
\hline $\mathrm{C}(9)-\mathrm{C}(10)$ & $1.373(9)$ & $C(42)-C(43)$ & $1.397(9)$ \\
\hline$C(10)-C(11)$ & $1.374(9)$ & $C(43)-C(44)$ & $1.385(10)$ \\
\hline$C(11)-C(12)$ & $1.382(9)$ & $C(44)-C(45)$ & $1.385(10)$ \\
\hline$C(13)-C(18)$ & $1.396(7)$ & $C(45)-C(46)$ & $1.385(9)$ \\
\hline$C(13)-C(14)$ & $1.406(8)$ & $\mathrm{S}(1)-\mathrm{O}(3)$ & $1.443(4)$ \\
\hline$C(14)-C(15)$ & $1.372(8)$ & $\mathrm{S}(1)-\mathrm{O}(4)$ & $1.447(4)$ \\
\hline$C(15)-C(16)$ & $1.377(8)$ & $\mathrm{S}(1)-\mathrm{O}(2)$ & $1.458(4)$ \\
\hline$C(16)-C(17)$ & $1.410(7)$ & $S(1)-C(47)$ & $1.804(8)$ \\
\hline$C(17)-C(18)$ & $1.386(7)$ & $\mathrm{C}(47)-\mathrm{F}(2)$ & $1.341(8)$ \\
\hline$C(17)-C(19)$ & $1.524(7)$ & $\mathrm{C}(47)-\mathrm{F}(1)$ & $1.342(7)$ \\
\hline$C(19)-C(22)$ & $1.522(8)$ & $\mathrm{C}(47)-\mathrm{F}(3)$ & $1.356(8)$ \\
\hline$C(19)-C(21)$ & $1.536(8)$ & $\operatorname{Pd}\left(1^{\prime}\right)-C\left(40^{\prime}\right)$ & $2.095(6)$ \\
\hline$C(19)-C(20)$ & $1.545(8)$ & $\operatorname{Pd}\left(1^{\prime}\right)-C\left(41^{\prime}\right)$ & $2.239(5)$ \\
\hline
\end{tabular}




\begin{tabular}{|c|c|c|c|}
\hline $\operatorname{Pd}\left(1^{\prime}\right)-\mathrm{P}\left(2^{\prime}\right)$ & $2.3095(15)$ & $C\left(24^{\prime}\right)-C\left(25^{\prime}\right)$ & $1.403(8)$ \\
\hline $\operatorname{Pd}\left(1^{\prime}\right)-\mathrm{P}\left(1^{\prime}\right)$ & $2.4057(16)$ & $\mathrm{C}\left(25^{\prime}\right)-\mathrm{C}\left(26^{\prime}\right)$ & $1.384(7)$ \\
\hline $\operatorname{Pd}\left(1^{\prime}\right)-C\left(42^{\prime}\right)$ & $2.584(6)$ & $\mathrm{C}\left(26^{\prime}\right)-\mathrm{C}\left(27^{\prime}\right)$ & $1.388(7)$ \\
\hline $\mathrm{P}\left(1^{\prime}\right)-\mathrm{C}\left(7^{\prime}\right)$ & $1.821(6)$ & $\mathrm{C}\left(28^{\prime}\right)-\mathrm{C}\left(29^{\prime}\right)$ & $1.387(8)$ \\
\hline $\mathrm{P}\left(1^{\prime}\right)-\mathrm{C}\left(1^{\prime}\right)$ & $1.823(5)$ & $\mathrm{C}\left(28^{\prime}\right)-\mathrm{C}\left(33^{\prime}\right)$ & $1.397(8)$ \\
\hline $\mathrm{P}\left(1^{\prime}\right)-\mathrm{C}\left(13^{\prime}\right)$ & $1.835(6)$ & $\mathrm{C}\left(29^{\prime}\right)-\mathrm{C}\left(30^{\prime}\right)$ & $1.380(8)$ \\
\hline $\mathrm{P}\left(2^{\prime}\right)-\mathrm{C}\left(34^{\prime}\right)$ & $1.834(6)$ & $\mathrm{C}\left(30^{\prime}\right)-\mathrm{C}\left(31^{\prime}\right)$ & $1.379(9)$ \\
\hline $\mathrm{P}\left(2^{\prime}\right)-\mathrm{C}\left(28^{\prime}\right)$ & $1.837(6)$ & $\mathrm{C}\left(31^{\prime}\right)-\mathrm{C}\left(32^{\prime}\right)$ & $1.385(9)$ \\
\hline$P\left(2^{\prime}\right)-C\left(26^{\prime}\right)$ & $1.842(5)$ & $\mathrm{C}\left(32^{\prime}\right)-\mathrm{C}\left(33^{\prime}\right)$ & $1.377(8)$ \\
\hline $\mathrm{O}\left(1^{\prime}\right)-\mathrm{C}\left(18^{\prime}\right)$ & $1.374(6)$ & $\mathrm{C}\left(34^{\prime}\right)-\mathrm{C}\left(39^{\prime}\right)$ & $1.376(8)$ \\
\hline $\mathrm{O}\left(1^{\prime}\right)-\mathrm{C}\left(27^{\prime}\right)$ & $1.376(6)$ & $\mathrm{C}\left(34^{\prime}\right)-\mathrm{C}\left(35^{\prime}\right)$ & $1.379(7)$ \\
\hline $\mathrm{C}\left(1^{\prime}\right)-\mathrm{C}\left(6^{\prime}\right)$ & $1.390(8)$ & $\mathrm{C}\left(35^{\prime}\right)-\mathrm{C}\left(36^{\prime}\right)$ & $1.396(8)$ \\
\hline $\mathrm{C}\left(1^{\prime}\right)-\mathrm{C}\left(2^{\prime}\right)$ & $1.405(8)$ & $\mathrm{C}\left(36^{\prime}\right)-\mathrm{C}\left(37^{\prime}\right)$ & $1.392(8)$ \\
\hline $\mathrm{C}\left(2^{\prime}\right)-\mathrm{C}\left(3^{\prime}\right)$ & $1.393(8)$ & $\mathrm{C}\left(37^{\prime}\right)-\mathrm{C}\left(38^{\prime}\right)$ & $1.385(8)$ \\
\hline $\mathrm{C}\left(3^{\prime}\right)-\mathrm{C}\left(4^{\prime}\right)$ & $1.383(9)$ & $\mathrm{C}\left(38^{\prime}\right)-\mathrm{C}\left(39^{\prime}\right)$ & $1.374(8)$ \\
\hline$C\left(4^{\prime}\right)-C\left(5^{\prime}\right)$ & $1.368(9)$ & $\mathrm{C}\left(40^{\prime}\right)-\mathrm{C}\left(41^{\prime}\right)$ & $1.430(8)$ \\
\hline$C\left(5^{\prime}\right)-C\left(6^{\prime}\right)$ & $1.384(8)$ & $\mathrm{C}\left(41^{\prime}\right)-\mathrm{C}\left(42^{\prime}\right)$ & $1.402(9)$ \\
\hline$C\left(7^{\prime}\right)-C\left(8^{\prime}\right)$ & $1.388(8)$ & $C\left(41^{\prime}\right)-C\left(46^{\prime}\right)$ & $1.435(9)$ \\
\hline$C\left(7^{\prime}\right)-C\left(12^{\prime}\right)$ & $1.403(8)$ & $\mathrm{C}\left(42^{\prime}\right)-\mathrm{C}\left(43^{\prime}\right)$ & $1.400(9)$ \\
\hline $\mathrm{C}\left(8^{\prime}\right)-\mathrm{C}\left(9^{\prime}\right)$ & $1.386(8)$ & $C\left(43^{\prime}\right)-C\left(44^{\prime}\right)$ & $1.372(9)$ \\
\hline $\mathrm{C}\left(9^{\prime}\right)-\mathrm{C}\left(10^{\prime}\right)$ & $1.371(9)$ & $C\left(44^{\prime}\right)-C\left(45^{\prime}\right)$ & $1.382(10)$ \\
\hline$C\left(10^{\prime}\right)-C\left(11^{\prime}\right)$ & $1.394(9)$ & $C\left(45^{\prime}\right)-C\left(46^{\prime}\right)$ & $1.363(9)$ \\
\hline $\mathrm{C}\left(11^{\prime}\right)-\mathrm{C}\left(12^{\prime}\right)$ & $1.382(9)$ & $\mathrm{S}\left(1^{\prime}\right)-\mathrm{O}\left(2^{\prime}\right)$ & $1.413(5)$ \\
\hline $\mathrm{C}\left(13^{\prime}\right)-\mathrm{C}\left(18^{\prime}\right)$ & $1.391(7)$ & $\mathrm{S}\left(1^{\prime}\right)-\mathrm{O}\left(4^{\prime}\right)$ & $1.431(5)$ \\
\hline $\mathrm{C}\left(13^{\prime}\right)-\mathrm{C}\left(14^{\prime}\right)$ & $1.402(7)$ & $\mathrm{S}\left(1^{\prime}\right)-\mathrm{O}\left(3^{\prime}\right)$ & $1.447(6)$ \\
\hline$C\left(14^{\prime}\right)-C\left(15^{\prime}\right)$ & $1.377(8)$ & $\mathrm{S}\left(1^{\prime}\right)-\mathrm{C}\left(47^{\prime}\right)$ & $1.632(13)$ \\
\hline$C\left(15^{\prime}\right)-C\left(16^{\prime}\right)$ & $1.387(8)$ & $\mathrm{C}\left(47^{\prime}\right)-\mathrm{F}\left(3^{\prime}\right)$ & $1.211(13)$ \\
\hline$C\left(16^{\prime}\right)-C\left(17^{\prime}\right)$ & $1.398(7)$ & $\mathrm{C}\left(47^{\prime}\right)-\mathrm{F}\left(1^{\prime}\right)$ & $1.510(19)$ \\
\hline $\mathrm{C}\left(17^{\prime}\right)-\mathrm{C}\left(18^{\prime}\right)$ & $1.386(7)$ & $\mathrm{C}\left(47^{\prime}\right)-\mathrm{F}\left(2^{\prime}\right)$ & $1.557(18)$ \\
\hline $\mathrm{C}\left(17^{\prime}\right)-\mathrm{C}\left(19^{\prime}\right)$ & $1.535(7)$ & & \\
\hline $\mathrm{C}\left(19^{\prime}\right)-\mathrm{C}\left(22^{\prime}\right)$ & $1.520(8)$ & $C(40)-P d(1)-C(41)$ & $38.9(2)$ \\
\hline $\mathrm{C}\left(19^{\prime}\right)-\mathrm{C}\left(21^{\prime}\right)$ & $1.555(8)$ & $\mathrm{C}(40)-\mathrm{Pd}(1)-\mathrm{P}(2)$ & $91.81(17)$ \\
\hline $\mathrm{C}\left(19^{\prime}\right)-\mathrm{C}\left(20^{\prime}\right)$ & $1.562(8)$ & $\mathrm{C}(41)-\mathrm{Pd}(1)-\mathrm{P}(2)$ & $130.11(16)$ \\
\hline $\mathrm{C}\left(22^{\prime}\right)-\mathrm{C}\left(23^{\prime}\right)$ & $1.388(8)$ & $\mathrm{C}(40)-\mathrm{Pd}(1)-\mathrm{P}(1)$ & $158.10(17)$ \\
\hline $\mathrm{C}\left(22^{\prime}\right)-\mathrm{C}\left(27^{\prime}\right)$ & $1.396(7)$ & $\mathrm{C}(41)-\mathrm{Pd}(1)-\mathrm{P}(1)$ & 119.74(17) \\
\hline $\mathrm{C}\left(23^{\prime}\right)-\mathrm{C}\left(24^{\prime}\right)$ & $1.366(8)$ & $\mathrm{P}(2)-\mathrm{Pd}(1)-\mathrm{P}(1)$ & $108.11(5)$ \\
\hline
\end{tabular}




\begin{tabular}{|c|c|c|c|}
\hline $\mathrm{C}(1)-\mathrm{P}(1)-\mathrm{C}(13)$ & $101.1(3)$ & $C(18)-C(17)-C(19)$ & $119.6(5)$ \\
\hline $\mathrm{C}(1)-\mathrm{P}(1)-\mathrm{C}(7)$ & $104.5(3)$ & $C(16)-C(17)-C(19)$ & $124.0(5)$ \\
\hline$C(13)-P(1)-C(7)$ & $104.4(3)$ & $\mathrm{O}(1)-\mathrm{C}(18)-\mathrm{C}(17)$ & $120.9(5)$ \\
\hline$C(1)-P(1)-P d(1)$ & $112.59(19)$ & $\mathrm{O}(1)-\mathrm{C}(18)-\mathrm{C}(13)$ & $114.1(5)$ \\
\hline$C(13)-P(1)-P d(1)$ & $123.02(18)$ & $C(17)-C(18)-C(13)$ & $124.9(5)$ \\
\hline$C(7)-P(1)-P d(1)$ & 109.47(19) & $C(22)-C(19)-C(17)$ & $108.7(4)$ \\
\hline$C(28)-P(2)-C(26)$ & $100.8(2)$ & $C(22)-C(19)-C(21)$ & $110.9(5)$ \\
\hline $\mathrm{C}(28)-\mathrm{P}(2)-\mathrm{C}(34)$ & $104.2(3)$ & $C(17)-C(19)-C(21)$ & $110.8(5)$ \\
\hline$C(26)-P(2)-C(34)$ & $104.6(3)$ & $C(22)-C(19)-C(20)$ & $109.2(5)$ \\
\hline $\mathrm{C}(28)-\mathrm{P}(2)-\mathrm{Pd}(1)$ & $116.96(18)$ & $C(17)-C(19)-C(20)$ & $108.1(5)$ \\
\hline$C(26)-P(2)-P d(1)$ & $123.13(18)$ & $C(21)-C(19)-C(20)$ & $109.0(5)$ \\
\hline $\mathrm{C}(34)-\mathrm{P}(2)-\mathrm{Pd}(1)$ & 105.12(19) & $\mathrm{C}(27)-\mathrm{C}(22)-\mathrm{C}(23)$ & $115.9(5)$ \\
\hline $\mathrm{C}(18)-\mathrm{O}(1)-\mathrm{C}(27)$ & $114.6(4)$ & $C(27)-C(22)-C(19)$ & $119.5(5)$ \\
\hline$C(6)-C(1)-C(2)$ & $117.5(6)$ & $\mathrm{C}(23)-\mathrm{C}(22)-\mathrm{C}(19)$ & $124.5(5)$ \\
\hline$C(6)-C(1)-P(1)$ & $118.6(5)$ & $\mathrm{C}(24)-\mathrm{C}(23)-\mathrm{C}(22)$ & $121.2(5)$ \\
\hline $\mathrm{C}(2)-\mathrm{C}(1)-\mathrm{P}(1)$ & $123.9(5)$ & $\mathrm{C}(23)-\mathrm{C}(24)-\mathrm{C}(25)$ & $121.3(5)$ \\
\hline$C(3)-C(2)-C(1)$ & $121.2(7)$ & $C(24)-C(25)-C(26)$ & $120.0(5)$ \\
\hline$C(2)-C(3)-C(4)$ & $119.9(7)$ & $C(27)-C(26)-C(25)$ & $116.9(5)$ \\
\hline$C(5)-C(4)-C(3)$ & $120.2(6)$ & $\mathrm{C}(27)-\mathrm{C}(26)-\mathrm{P}(2)$ & $119.2(4)$ \\
\hline$C(4)-C(5)-C(6)$ & $120.1(7)$ & $\mathrm{C}(25)-\mathrm{C}(26)-\mathrm{P}(2)$ & $123.9(4)$ \\
\hline$C(1)-C(6)-C(5)$ & $121.1(7)$ & $\mathrm{C}(26)-\mathrm{C}(27)-\mathrm{O}(1)$ & $114.9(5)$ \\
\hline$C(8)-C(7)-C(12)$ & $118.5(6)$ & $C(26)-C(27)-C(22)$ & $124.6(5)$ \\
\hline $\mathrm{C}(8)-\mathrm{C}(7)-\mathrm{P}(1)$ & $123.2(5)$ & $\mathrm{O}(1)-\mathrm{C}(27)-\mathrm{C}(22)$ & $120.5(5)$ \\
\hline $\mathrm{C}(12)-\mathrm{C}(7)-\mathrm{P}(1)$ & $118.3(5)$ & $C(29)-C(28)-C(33)$ & $119.2(6)$ \\
\hline$C(7)-C(8)-C(9)$ & $120.2(6)$ & $\mathrm{C}(29)-\mathrm{C}(28)-\mathrm{P}(2)$ & $122.4(5)$ \\
\hline$C(10)-C(9)-C(8)$ & $120.4(6)$ & $\mathrm{C}(33)-\mathrm{C}(28)-\mathrm{P}(2)$ & $118.3(4)$ \\
\hline$C(9)-C(10)-C(11)$ & $119.7(6)$ & $\mathrm{C}(28)-\mathrm{C}(29)-\mathrm{C}(30)$ & $118.6(6)$ \\
\hline$C(10)-C(11)-C(12)$ & $120.6(6)$ & $C(31)-C(30)-C(29)$ & $122.3(7)$ \\
\hline$C(11)-C(12)-C(7)$ & $120.5(6)$ & $\mathrm{C}(32)-\mathrm{C}(31)-\mathrm{C}(30)$ & $119.2(6)$ \\
\hline$C(18)-C(13)-C(14)$ & $116.6(5)$ & $\mathrm{C}(31)-\mathrm{C}(32)-\mathrm{C}(33)$ & $120.9(7)$ \\
\hline $\mathrm{C}(18)-\mathrm{C}(13)-\mathrm{P}(1)$ & $117.6(4)$ & $\mathrm{C}(32)-\mathrm{C}(33)-\mathrm{C}(28)$ & $119.9(6)$ \\
\hline $\mathrm{C}(14)-\mathrm{C}(13)-\mathrm{P}(1)$ & $125.7(4)$ & $C(35)-C(34)-C(39)$ & $119.6(6)$ \\
\hline$C(15)-C(14)-C(13)$ & $119.5(5)$ & $\mathrm{C}(35)-\mathrm{C}(34)-\mathrm{P}(2)$ & $122.9(5)$ \\
\hline$C(14)-C(15)-C(16)$ & $122.9(5)$ & $\mathrm{C}(39)-\mathrm{C}(34)-\mathrm{P}(2)$ & $117.4(5)$ \\
\hline$C(15)-C(16)-C(17)$ & $119.7(5)$ & $\mathrm{C}(34)-\mathrm{C}(35)-\mathrm{C}(36)$ & $121.0(7)$ \\
\hline$C(18)-C(17)-C(16)$ & $116.3(5)$ & $C(37)-C(36)-C(35)$ & $120.1(8)$ \\
\hline
\end{tabular}




\begin{tabular}{|c|c|c|c|}
\hline $\mathrm{C}(36)-\mathrm{C}(37)-\mathrm{C}(38)$ & $119.4(7)$ & $\mathrm{P}\left(1^{\prime}\right)-\mathrm{Pd}\left(1^{\prime}\right)-\mathrm{C}\left(42^{\prime}\right)$ & $98.49(14)$ \\
\hline $\mathrm{C}(39)-\mathrm{C}(38)-\mathrm{C}(37)$ & $120.5(8)$ & $\mathrm{C}\left(7^{\prime}\right)-\mathrm{P}\left(1^{\prime}\right)-\mathrm{C}\left(1^{\prime}\right)$ & $104.4(3)$ \\
\hline$C(38)-C(39)-C(34)$ & $119.3(7)$ & $\mathrm{C}\left(7^{\prime}\right)-\mathrm{P}\left(1^{\prime}\right)-\mathrm{C}\left(13^{\prime}\right)$ & $105.9(3)$ \\
\hline$C(41)-C(40)-P d(1)$ & $76.3(3)$ & $\mathrm{C}\left(1^{\prime}\right)-\mathrm{P}\left(1^{\prime}\right)-\mathrm{C}\left(13^{\prime}\right)$ & $98.6(2)$ \\
\hline$C(46)-C(41)-C(42)$ & $118.3(6)$ & $C\left(7^{\prime}\right)-P\left(1^{\prime}\right)-P d\left(1^{\prime}\right)$ & $109.88(18)$ \\
\hline$C(46)-C(41)-C(40)$ & $120.0(6)$ & $\mathrm{C}\left(1^{\prime}\right)-\mathrm{P}\left(1^{\prime}\right)-\mathrm{Pd}\left(1^{\prime}\right)$ & $113.70(19)$ \\
\hline$C(42)-C(41)-C(40)$ & $121.4(6)$ & $C\left(13^{\prime}\right)-P\left(1^{\prime}\right)-P d\left(1^{\prime}\right)$ & $122.54(18)$ \\
\hline$C(46)-C(41)-P d(1)$ & $92.3(4)$ & $\mathrm{C}\left(34^{\prime}\right)-\mathrm{P}\left(2^{\prime}\right)-\mathrm{C}\left(28^{\prime}\right)$ & $104.9(3)$ \\
\hline$C(42)-C(41)-P d(1)$ & $107.7(4)$ & $\mathrm{C}\left(34^{\prime}\right)-\mathrm{P}\left(2^{\prime}\right)-\mathrm{C}\left(26^{\prime}\right)$ & $105.5(2)$ \\
\hline$C(40)-C(41)-P d(1)$ & $64.8(3)$ & $\mathrm{C}\left(28^{\prime}\right)-\mathrm{P}\left(2^{\prime}\right)-\mathrm{C}\left(26^{\prime}\right)$ & $101.5(2)$ \\
\hline$C(43)-C(42)-C(41)$ & $120.0(7)$ & $\mathrm{C}\left(34^{\prime}\right)-\mathrm{P}\left(2^{\prime}\right)-\mathrm{Pd}\left(1^{\prime}\right)$ & $106.74(17)$ \\
\hline$C(44)-C(43)-C(42)$ & $119.6(7)$ & $\mathrm{C}\left(28^{\prime}\right)-\mathrm{P}\left(2^{\prime}\right)-\mathrm{Pd}\left(1^{\prime}\right)$ & $116.10(18)$ \\
\hline$C(45)-C(44)-C(43)$ & $121.7(7)$ & $\mathrm{C}\left(26^{\prime}\right)-\mathrm{P}\left(2^{\prime}\right)-\mathrm{Pd}\left(1^{\prime}\right)$ & $120.63(18)$ \\
\hline$C(46)-C(45)-C(44)$ & $118.2(7)$ & $\mathrm{C}\left(18^{\prime}\right)-\mathrm{O}\left(1^{\prime}\right)-\mathrm{C}\left(27^{\prime}\right)$ & $116.0(4)$ \\
\hline$C(45)-C(46)-C(41)$ & $122.1(7)$ & $\mathrm{C}\left(6^{\prime}\right)-\mathrm{C}\left(1^{\prime}\right)-\mathrm{C}\left(2^{\prime}\right)$ & $119.4(5)$ \\
\hline $\mathrm{O}(3)-\mathrm{S}(1)-\mathrm{O}(4)$ & $114.5(3)$ & $\mathrm{C}\left(6^{\prime}\right)-\mathrm{C}\left(1^{\prime}\right)-\mathrm{P}\left(1^{\prime}\right)$ & $116.1(4)$ \\
\hline $\mathrm{O}(3)-\mathrm{S}(1)-\mathrm{O}(2)$ & $115.8(3)$ & $\mathrm{C}\left(2^{\prime}\right)-\mathrm{C}\left(1^{\prime}\right)-\mathrm{P}\left(1^{\prime}\right)$ & $124.0(5)$ \\
\hline $\mathrm{O}(4)-\mathrm{S}(1)-\mathrm{O}(2)$ & $115.3(3)$ & $\mathrm{C}\left(3^{\prime}\right)-\mathrm{C}\left(2^{\prime}\right)-\mathrm{C}\left(1^{\prime}\right)$ & $118.9(6)$ \\
\hline $\mathrm{O}(3)-\mathrm{S}(1)-\mathrm{C}(47)$ & $103.2(3)$ & $\mathrm{C}\left(4^{\prime}\right)-\mathrm{C}\left(3^{\prime}\right)-\mathrm{C}\left(2^{\prime}\right)$ & $120.6(6)$ \\
\hline $\mathrm{O}(4)-\mathrm{S}(1)-\mathrm{C}(47)$ & $103.5(3)$ & $C\left(5^{\prime}\right)-C\left(4^{\prime}\right)-C\left(3^{\prime}\right)$ & $120.4(6)$ \\
\hline $\mathrm{O}(2)-\mathrm{S}(1)-\mathrm{C}(47)$ & $101.9(3)$ & $C\left(4^{\prime}\right)-C\left(5^{\prime}\right)-C\left(6^{\prime}\right)$ & $120.1(6)$ \\
\hline $\mathrm{F}(2)-\mathrm{C}(47)-\mathrm{F}(1)$ & $106.4(6)$ & $\mathrm{C}\left(5^{\prime}\right)-\mathrm{C}\left(6^{\prime}\right)-\mathrm{C}\left(1^{\prime}\right)$ & $120.6(6)$ \\
\hline $\mathrm{F}(2)-\mathrm{C}(47)-\mathrm{F}(3)$ & $108.4(6)$ & $\mathrm{C}\left(8^{\prime}\right)-\mathrm{C}\left(7^{\prime}\right)-\mathrm{C}\left(12^{\prime}\right)$ & 119.1(6) \\
\hline $\mathrm{F}(1)-\mathrm{C}(47)-\mathrm{F}(3)$ & $108.5(6)$ & $\mathrm{C}\left(8^{\prime}\right)-\mathrm{C}\left(7^{\prime}\right)-\mathrm{P}\left(1^{\prime}\right)$ & $123.0(5)$ \\
\hline $\mathrm{F}(2)-\mathrm{C}(47)-\mathrm{S}(1)$ & $111.2(5)$ & $\mathrm{C}\left(12^{\prime}\right)-\mathrm{C}\left(7^{\prime}\right)-\mathrm{P}\left(1^{\prime}\right)$ & $117.9(5)$ \\
\hline $\mathrm{F}(1)-\mathrm{C}(47)-\mathrm{S}(1)$ & $112.2(5)$ & $\mathrm{C}\left(9^{\prime}\right)-\mathrm{C}\left(8^{\prime}\right)-\mathrm{C}\left(7^{\prime}\right)$ & $120.1(6)$ \\
\hline $\mathrm{F}(3)-\mathrm{C}(47)-\mathrm{S}(1)$ & $110.0(5)$ & $\mathrm{C}\left(10^{\prime}\right)-\mathrm{C}\left(9^{\prime}\right)-\mathrm{C}\left(8^{\prime}\right)$ & $120.3(6)$ \\
\hline $\mathrm{C}\left(40^{\prime}\right)-\mathrm{Pd}\left(1^{\prime}\right)-\mathrm{C}\left(41^{\prime}\right)$ & $38.3(2)$ & $\mathrm{C}\left(9^{\prime}\right)-\mathrm{C}\left(10^{\prime}\right)-\mathrm{C}\left(11^{\prime}\right)$ & $120.8(6)$ \\
\hline $\mathrm{C}\left(40^{\prime}\right)-\mathrm{Pd}\left(1^{\prime}\right)-\mathrm{P}\left(2^{\prime}\right)$ & $90.62(16)$ & $C\left(12^{\prime}\right)-C\left(11^{\prime}\right)-C\left(10^{\prime}\right)$ & $118.9(6)$ \\
\hline $\mathrm{C}\left(41^{\prime}\right)-\mathrm{Pd}\left(1^{\prime}\right)-\mathrm{P}\left(2^{\prime}\right)$ & $128.92(16)$ & $\mathrm{C}\left(11^{\prime}\right)-\mathrm{C}\left(12^{\prime}\right)-\mathrm{C}\left(7^{\prime}\right)$ & $120.8(6)$ \\
\hline$C\left(40^{\prime}\right)-\mathrm{Pd}\left(1^{\prime}\right)-\mathrm{P}\left(1^{\prime}\right)$ & $160.38(17)$ & $C\left(18^{\prime}\right)-C\left(13^{\prime}\right)-C\left(14^{\prime}\right)$ & $117.0(5)$ \\
\hline$C\left(41^{\prime}\right)-P d\left(1^{\prime}\right)-P\left(1^{\prime}\right)$ & $123.37(16)$ & $\mathrm{C}\left(18^{\prime}\right)-\mathrm{C}\left(13^{\prime}\right)-\mathrm{P}\left(1^{\prime}\right)$ & $118.6(4)$ \\
\hline $\mathrm{P}\left(2^{\prime}\right)-\mathrm{Pd}\left(1^{\prime}\right)-\mathrm{P}\left(1^{\prime}\right)$ & $107.45(5)$ & $\mathrm{C}\left(14^{\prime}\right)-\mathrm{C}\left(13^{\prime}\right)-\mathrm{P}\left(1^{\prime}\right)$ & $124.0(4)$ \\
\hline$C\left(40^{\prime}\right)-\operatorname{Pd}\left(1^{\prime}\right)-C\left(42^{\prime}\right)$ & $62.0(2)$ & $\mathrm{C}\left(15^{\prime}\right)-\mathrm{C}\left(14^{\prime}\right)-\mathrm{C}\left(13^{\prime}\right)$ & $120.5(5)$ \\
\hline $\mathrm{C}\left(41^{\prime}\right)-\mathrm{Pd}\left(1^{\prime}\right)-\mathrm{C}\left(42^{\prime}\right)$ & $32.8(2)$ & $C\left(14^{\prime}\right)-C\left(15^{\prime}\right)-C\left(16^{\prime}\right)$ & $120.5(5)$ \\
\hline $\mathrm{P}\left(2^{\prime}\right)-\mathrm{Pd}\left(1^{\prime}\right)-\mathrm{C}\left(42^{\prime}\right)$ & $141.61(15)$ & $\mathrm{C}\left(15^{\prime}\right)-\mathrm{C}\left(16^{\prime}\right)-\mathrm{C}\left(17^{\prime}\right)$ & $121.1(5)$ \\
\hline
\end{tabular}




\begin{tabular}{|c|c|c|c|}
\hline $\mathrm{C}\left(18^{\prime}\right)-\mathrm{C}\left(17^{\prime}\right)-\mathrm{C}\left(16^{\prime}\right)$ & $116.5(5)$ & $\mathrm{C}\left(37^{\prime}\right)-\mathrm{C}\left(36^{\prime}\right)-\mathrm{C}\left(35^{\prime}\right)$ & $118.8(5)$ \\
\hline $\mathrm{C}\left(18^{\prime}\right)-\mathrm{C}\left(17^{\prime}\right)-\mathrm{C}\left(19^{\prime}\right)$ & $120.3(5)$ & $\mathrm{C}\left(38^{\prime}\right)-\mathrm{C}\left(37^{\prime}\right)-\mathrm{C}\left(36^{\prime}\right)$ & $120.0(6)$ \\
\hline $\mathrm{C}\left(16^{\prime}\right)-\mathrm{C}\left(17^{\prime}\right)-\mathrm{C}\left(19^{\prime}\right)$ & $123.3(5)$ & $\mathrm{C}\left(39^{\prime}\right)-\mathrm{C}\left(38^{\prime}\right)-\mathrm{C}\left(37^{\prime}\right)$ & $120.4(6)$ \\
\hline $\mathrm{O}\left(1^{\prime}\right)-\mathrm{C}\left(18^{\prime}\right)-\mathrm{C}\left(17^{\prime}\right)$ & $121.5(5)$ & $\mathrm{C}\left(38^{\prime}\right)-\mathrm{C}\left(39^{\prime}\right)-\mathrm{C}\left(34^{\prime}\right)$ & $120.3(5)$ \\
\hline $\mathrm{O}\left(1^{\prime}\right)-\mathrm{C}\left(18^{\prime}\right)-\mathrm{C}\left(13^{\prime}\right)$ & $114.3(5)$ & $\mathrm{C}\left(41^{\prime}\right)-\mathrm{C}\left(40^{\prime}\right)-\mathrm{Pd}\left(1^{\prime}\right)$ & $76.3(3)$ \\
\hline$C\left(17^{\prime}\right)-C\left(18^{\prime}\right)-C\left(13^{\prime}\right)$ & $124.2(5)$ & $C\left(42^{\prime}\right)-C\left(41^{\prime}\right)-C\left(40^{\prime}\right)$ & $119.4(6)$ \\
\hline$C\left(22^{\prime}\right)-C\left(19^{\prime}\right)-C\left(17^{\prime}\right)$ & 109.3(4) & $C\left(42^{\prime}\right)-C\left(41^{\prime}\right)-C\left(46^{\prime}\right)$ & $117.8(6)$ \\
\hline$C\left(22^{\prime}\right)-C\left(19^{\prime}\right)-C\left(21^{\prime}\right)$ & $110.5(5)$ & $\mathrm{C}\left(40^{\prime}\right)-\mathrm{C}\left(41^{\prime}\right)-\mathrm{C}\left(46^{\prime}\right)$ & $122.5(6)$ \\
\hline$C\left(17^{\prime}\right)-C\left(19^{\prime}\right)-C\left(21^{\prime}\right)$ & 108.1(5) & $C\left(42^{\prime}\right)-C\left(41^{\prime}\right)-\operatorname{Pd}\left(1^{\prime}\right)$ & $87.3(4)$ \\
\hline $\mathrm{C}\left(22^{\prime}\right)-\mathrm{C}\left(19^{\prime}\right)-\mathrm{C}\left(20^{\prime}\right)$ & $110.7(5)$ & $\mathrm{C}\left(40^{\prime}\right)-\mathrm{C}\left(41^{\prime}\right)-\operatorname{Pd}\left(1^{\prime}\right)$ & $65.4(3)$ \\
\hline$C\left(17^{\prime}\right)-C\left(19^{\prime}\right)-C\left(20^{\prime}\right)$ & $109.7(5)$ & $\mathrm{C}\left(46^{\prime}\right)-\mathrm{C}\left(41^{\prime}\right)-\mathrm{Pd}\left(1^{\prime}\right)$ & $112.7(4)$ \\
\hline $\mathrm{C}\left(21^{\prime}\right)-\mathrm{C}\left(19^{\prime}\right)-\mathrm{C}\left(20^{\prime}\right)$ & $108.6(5)$ & $\mathrm{C}\left(43^{\prime}\right)-\mathrm{C}\left(42^{\prime}\right)-\mathrm{C}\left(41^{\prime}\right)$ & $120.3(6)$ \\
\hline$C\left(23^{\prime}\right)-C\left(22^{\prime}\right)-C\left(27^{\prime}\right)$ & $115.9(5)$ & $\mathrm{C}\left(43^{\prime}\right)-\mathrm{C}\left(42^{\prime}\right)-\mathrm{Pd}\left(1^{\prime}\right)$ & $126.3(5)$ \\
\hline$C\left(23^{\prime}\right)-C\left(22^{\prime}\right)-C\left(19^{\prime}\right)$ & $124.1(5)$ & $\mathrm{C}\left(41^{\prime}\right)-\mathrm{C}\left(42^{\prime}\right)-\mathrm{Pd}\left(1^{\prime}\right)$ & $59.9(3)$ \\
\hline $\mathrm{C}\left(27^{\prime}\right)-\mathrm{C}\left(22^{\prime}\right)-\mathrm{C}\left(19^{\prime}\right)$ & $120.0(5)$ & $\mathrm{C}\left(44^{\prime}\right)-\mathrm{C}\left(43^{\prime}\right)-\mathrm{C}\left(42^{\prime}\right)$ & $120.2(7)$ \\
\hline $\mathrm{C}\left(24^{\prime}\right)-\mathrm{C}\left(23^{\prime}\right)-\mathrm{C}\left(22^{\prime}\right)$ & $122.6(5)$ & $\mathrm{C}\left(43^{\prime}\right)-\mathrm{C}\left(44^{\prime}\right)-\mathrm{C}\left(45^{\prime}\right)$ & $120.4(6)$ \\
\hline$C\left(23^{\prime}\right)-C\left(24^{\prime}\right)-C\left(25^{\prime}\right)$ & $120.2(5)$ & $\mathrm{C}\left(46^{\prime}\right)-\mathrm{C}\left(45^{\prime}\right)-\mathrm{C}\left(44^{\prime}\right)$ & $121.0(7)$ \\
\hline$C\left(26^{\prime}\right)-C\left(25^{\prime}\right)-C\left(24^{\prime}\right)$ & $119.3(5)$ & $\mathrm{C}\left(45^{\prime}\right)-\mathrm{C}\left(46^{\prime}\right)-\mathrm{C}\left(41^{\prime}\right)$ & $120.2(7)$ \\
\hline$C\left(25^{\prime}\right)-C\left(26^{\prime}\right)-C\left(27^{\prime}\right)$ & $118.7(5)$ & $\mathrm{O}\left(2^{\prime}\right)-\mathrm{S}\left(1^{\prime}\right)-\mathrm{O}\left(4^{\prime}\right)$ & $115.5(4)$ \\
\hline $\mathrm{C}\left(25^{\prime}\right)-\mathrm{C}\left(26^{\prime}\right)-\mathrm{P}\left(2^{\prime}\right)$ & $124.4(4)$ & $\mathrm{O}\left(2^{\prime}\right)-\mathrm{S}\left(1^{\prime}\right)-\mathrm{O}\left(3^{\prime}\right)$ & $115.5(4)$ \\
\hline$C\left(27^{\prime}\right)-C\left(26^{\prime}\right)-\mathrm{P}\left(2^{\prime}\right)$ & $117.0(4)$ & $\mathrm{O}\left(4^{\prime}\right)-\mathrm{S}\left(1^{\prime}\right)-\mathrm{O}\left(3^{\prime}\right)$ & $117.6(4)$ \\
\hline $\mathrm{O}\left(1^{\prime}\right)-\mathrm{C}\left(27^{\prime}\right)-\mathrm{C}\left(26^{\prime}\right)$ & $115.1(5)$ & $\mathrm{O}\left(2^{\prime}\right)-\mathrm{S}\left(1^{\prime}\right)-\mathrm{C}\left(47^{\prime}\right)$ & $96.7(7)$ \\
\hline $\mathrm{O}\left(1^{\prime}\right)-\mathrm{C}\left(27^{\prime}\right)-\mathrm{C}\left(22^{\prime}\right)$ & $121.6(5)$ & $\mathrm{O}\left(4^{\prime}\right)-\mathrm{S}\left(1^{\prime}\right)-\mathrm{C}\left(47^{\prime}\right)$ & $95.2(6)$ \\
\hline $\mathrm{C}\left(26^{\prime}\right)-\mathrm{C}\left(27^{\prime}\right)-\mathrm{C}\left(22^{\prime}\right)$ & $123.3(5)$ & $\mathrm{O}\left(3^{\prime}\right)-\mathrm{S}\left(1^{\prime}\right)-\mathrm{C}\left(47^{\prime}\right)$ & $111.9(9)$ \\
\hline $\mathrm{C}\left(29^{\prime}\right)-\mathrm{C}\left(28^{\prime}\right)-\mathrm{C}\left(33^{\prime}\right)$ & $118.4(5)$ & $\mathrm{F}\left(3^{\prime}\right)-\mathrm{C}\left(47^{\prime}\right)-\mathrm{F}\left(1^{\prime}\right)$ & $102.8(10)$ \\
\hline $\mathrm{C}\left(29^{\prime}\right)-\mathrm{C}\left(28^{\prime}\right)-\mathrm{P}\left(2^{\prime}\right)$ & $121.8(4)$ & $\mathrm{F}\left(3^{\prime}\right)-\mathrm{C}\left(47^{\prime}\right)-\mathrm{F}\left(2^{\prime}\right)$ & $103.5(10)$ \\
\hline $\mathrm{C}\left(33^{\prime}\right)-\mathrm{C}\left(28^{\prime}\right)-\mathrm{P}\left(2^{\prime}\right)$ & $119.8(5)$ & $\mathrm{F}\left(1^{\prime}\right)-\mathrm{C}\left(47^{\prime}\right)-\mathrm{F}\left(2^{\prime}\right)$ & $87.2(15)$ \\
\hline $\mathrm{C}\left(30^{\prime}\right)-\mathrm{C}\left(29^{\prime}\right)-\mathrm{C}\left(28^{\prime}\right)$ & $120.0(6)$ & $\mathrm{F}\left(3^{\prime}\right)-\mathrm{C}\left(47^{\prime}\right)-\mathrm{S}\left(1^{\prime}\right)$ & $133(2)$ \\
\hline $\mathrm{C}\left(31^{\prime}\right)-\mathrm{C}\left(30^{\prime}\right)-\mathrm{C}\left(29^{\prime}\right)$ & $121.4(6)$ & $\mathrm{F}\left(1^{\prime}\right)-\mathrm{C}\left(47^{\prime}\right)-\mathrm{S}\left(1^{\prime}\right)$ & $110.6(8)$ \\
\hline$C\left(30^{\prime}\right)-C\left(31^{\prime}\right)-C\left(32^{\prime}\right)$ & $119.1(6)$ & $\mathrm{F}\left(2^{\prime}\right)-\mathrm{C}\left(47^{\prime}\right)-\mathrm{S}\left(1^{\prime}\right)$ & $110.0(7)$ \\
\hline$C\left(33^{\prime}\right)-C\left(32^{\prime}\right)-C\left(31^{\prime}\right)$ & $119.9(6)$ & & \\
\hline $\mathrm{C}\left(32^{\prime}\right)-\mathrm{C}\left(33^{\prime}\right)-\mathrm{C}\left(28^{\prime}\right)$ & $121.2(6)$ & & \\
\hline $\mathrm{C}\left(39^{\prime}\right)-\mathrm{C}\left(34^{\prime}\right)-\mathrm{C}\left(35^{\prime}\right)$ & $119.9(6)$ & & \\
\hline $\mathrm{C}\left(39^{\prime}\right)-\mathrm{C}\left(34^{\prime}\right)-\mathrm{P}\left(2^{\prime}\right)$ & $118.0(4)$ & & \\
\hline $\mathrm{C}\left(35^{\prime}\right)-\mathrm{C}\left(34^{\prime}\right)-\mathrm{P}\left(2^{\prime}\right)$ & $122.1(4)$ & & \\
\hline $\mathrm{C}\left(34^{\prime}\right)-\mathrm{C}\left(35^{\prime}\right)-\mathrm{C}\left(36^{\prime}\right)$ & $120.6(5)$ & & \\
\hline
\end{tabular}


Table S9. Anisotropic displacement parameters $\left(\AA^{2} \times 1^{3}\right)$ for 6 . The anisotropic displacement factor exponent takes the form: $-2 \pi^{2}\left[h^{2} a^{* 2} U^{11}+\ldots+2 h k a^{*} b^{*} U^{12}\right]$

\begin{tabular}{|c|c|c|c|c|c|c|}
\hline & $\mathrm{U}^{11}$ & $\mathrm{U}^{22}$ & $\mathrm{U}^{33}$ & $\mathrm{U}^{23}$ & $\mathrm{U}^{13}$ & $\mathrm{U}^{12}$ \\
\hline $\operatorname{Pd}(1)$ & $18(1)$ & $18(1)$ & $16(1)$ & $3(1)$ & 1(1) & $0(1)$ \\
\hline $\mathrm{P}(1)$ & $17(1)$ & $16(1)$ & $17(1)$ & 1(1) & $2(1)$ & $1(1)$ \\
\hline $\mathrm{P}(2)$ & $16(1)$ & $15(1)$ & $17(1)$ & $1(1)$ & $0(1)$ & $-1(1)$ \\
\hline $\mathrm{O}(1)$ & $12(2)$ & $22(2)$ & $17(2)$ & $-2(2)$ & $2(2)$ & $0(2)$ \\
\hline $\mathrm{C}(1)$ & $22(3)$ & 21(4) & $16(3)$ & $1(2)$ & 2(2) & $-4(3)$ \\
\hline$C(2)$ & $31(4)$ & $45(5)$ & $23(3)$ & $3(3)$ & $8(3)$ & $5(3)$ \\
\hline$C(3)$ & $28(4)$ & $66(6)$ & 41(4) & $7(4)$ & $15(3)$ & $10(4)$ \\
\hline $\mathrm{C}(4)$ & $28(4)$ & $106(8)$ & $18(4)$ & $-3(4)$ & $6(3)$ & $-22(5)$ \\
\hline$C(5)$ & $50(5)$ & $44(5)$ & $25(4)$ & $-3(3)$ & $14(3)$ & $-25(4)$ \\
\hline$C(6)$ & 41(4) & $30(4)$ & $20(3)$ & $-1(3)$ & $4(3)$ & $-9(3)$ \\
\hline$C(7)$ & $15(3)$ & $20(3)$ & $24(3)$ & $-2(3)$ & $3(2)$ & 1(3) \\
\hline $\mathrm{C}(8)$ & $25(3)$ & $25(4)$ & $28(3)$ & $7(3)$ & $13(3)$ & $6(3)$ \\
\hline $\mathrm{C}(9)$ & $35(4)$ & $23(4)$ & $29(4)$ & 13(3) & $12(3)$ & $6(3)$ \\
\hline $\mathrm{C}(10)$ & $26(3)$ & $17(4)$ & $51(4)$ & $0(3)$ & $-3(3)$ & $-1(3)$ \\
\hline $\mathrm{C}(11)$ & $51(5)$ & $15(4)$ & $44(4)$ & $-12(3)$ & $-22(4)$ & 1(3) \\
\hline$C(12)$ & 41(4) & $27(4)$ & $34(4)$ & $-5(3)$ & $-12(3)$ & $8(3)$ \\
\hline$C(13)$ & $17(3)$ & 11(3) & $24(3)$ & $3(2)$ & $2(3)$ & $6(2)$ \\
\hline$C(14)$ & $18(3)$ & $22(4)$ & $25(3)$ & $5(3)$ & $10(3)$ & $5(3)$ \\
\hline$C(15)$ & $12(3)$ & 19(3) & $25(3)$ & $0(3)$ & $-3(3)$ & $-6(2)$ \\
\hline$C(16)$ & 21(3) & $23(4)$ & $20(3)$ & $5(3)$ & $-6(3)$ & $-1(3)$ \\
\hline$C(17)$ & $17(3)$ & $14(3)$ & $17(3)$ & $4(2)$ & $-1(2)$ & $-1(3)$ \\
\hline$C(18)$ & $16(3)$ & $12(3)$ & $17(3)$ & $4(2)$ & $0(2)$ & $2(2)$ \\
\hline$C(19)$ & $19(3)$ & $24(4)$ & $18(3)$ & $-8(3)$ & $-1(3)$ & $0(3)$ \\
\hline$C(20)$ & 19(3) & $62(5)$ & 21(3) & 11(3) & $1(3)$ & $-2(3)$ \\
\hline$C(21)$ & $17(3)$ & $65(6)$ & $37(4)$ & $-25(4)$ & 4(3) & $-2(3)$ \\
\hline$C(22)$ & $17(3)$ & $20(3)$ & $18(3)$ & $3(3)$ & $0(2)$ & $-2(3)$ \\
\hline$C(23)$ & $27(3)$ & $20(4)$ & $20(3)$ & $3(3)$ & $6(3)$ & 2(3) \\
\hline$C(24)$ & $23(3)$ & $30(4)$ & $25(3)$ & 2(3) & 11(3) & $6(3)$ \\
\hline$C(25)$ & $17(3)$ & $24(4)$ & $20(3)$ & 3(3) & $0(3)$ & $0(3)$ \\
\hline$C(26)$ & $17(3)$ & $15(3)$ & $18(3)$ & $2(2)$ & $0(2)$ & $-4(2)$ \\
\hline
\end{tabular}




\begin{tabular}{|c|c|c|c|c|c|c|}
\hline$C(27)$ & $14(3)$ & $13(3)$ & $20(3)$ & $1(2)$ & $4(2)$ & $-4(2)$ \\
\hline$C(28)$ & $24(3)$ & $22(4)$ & $17(3)$ & $-9(3)$ & $4(3)$ & $-8(3)$ \\
\hline$C(29)$ & $25(3)$ & $31(4)$ & $34(4)$ & $5(3)$ & $-5(3)$ & $-4(3)$ \\
\hline$C(30)$ & $23(4)$ & $64(6)$ & $51(5)$ & $-6(4)$ & $-1(4)$ & $-18(4)$ \\
\hline$C(31)$ & $27(4)$ & $70(6)$ & $24(4)$ & $-7(4)$ & $-6(3)$ & $14(4)$ \\
\hline$C(32)$ & $34(4)$ & $40(4)$ & $19(3)$ & $-5(3)$ & $-2(3)$ & $15(3)$ \\
\hline$C(33)$ & $27(3)$ & $24(4)$ & $18(3)$ & $-2(3)$ & $-1(3)$ & $4(3)$ \\
\hline$C(34)$ & $13(3)$ & $17(3)$ & $41(4)$ & $4(3)$ & $4(3)$ & $-3(3)$ \\
\hline$C(35)$ & $29(4)$ & $38(5)$ & $41(4)$ & $17(3)$ & $-13(3)$ & $-9(3)$ \\
\hline$C(36)$ & $33(4)$ & $42(5)$ & $77(6)$ & $39(5)$ & $-21(4)$ & $-12(4)$ \\
\hline$C(37)$ & $29(4)$ & $27(5)$ & 112(8) & $27(5)$ & $19(5)$ & $3(4)$ \\
\hline$C(38)$ & $60(5)$ & $26(5)$ & $91(7)$ & $-11(4)$ & $45(5)$ & $-15(4)$ \\
\hline C(39) & $68(5)$ & $17(4)$ & $53(5)$ & $-3(3)$ & $39(4)$ & $-8(4)$ \\
\hline$C(40)$ & $31(4)$ & $31(4)$ & $19(3)$ & $2(3)$ & $-4(3)$ & $-4(3)$ \\
\hline$C(41)$ & $34(4)$ & $32(4)$ & $9(3)$ & $4(3)$ & $-2(3)$ & $-4(3)$ \\
\hline$C(42)$ & $40(4)$ & $45(5)$ & $25(4)$ & $-1(3)$ & $5(3)$ & $-4(4)$ \\
\hline$C(43)$ & $53(5)$ & $55(5)$ & $28(4)$ & $7(4)$ & $2(4)$ & $-23(4)$ \\
\hline$C(44)$ & $36(4)$ & $82(7)$ & $27(4)$ & $0(4)$ & $8(3)$ & $9(5)$ \\
\hline$C(45)$ & $47(4)$ & $53(5)$ & $28(4)$ & $-1(3)$ & $12(3)$ & $4(4)$ \\
\hline$C(46)$ & $41(4)$ & $45(5)$ & $20(3)$ & $-1(3)$ & $6(3)$ & $-3(4)$ \\
\hline $\mathrm{S}(1)$ & $30(1)$ & $30(1)$ & $27(1)$ & $2(1)$ & $2(1)$ & $0(1)$ \\
\hline $\mathrm{O}(2)$ & $57(3)$ & $55(3)$ & $29(3)$ & $-1(2)$ & $24(2)$ & $14(3)$ \\
\hline $\mathrm{O}(3)$ & $27(2)$ & $36(3)$ & $23(2)$ & $0(2)$ & $-2(2)$ & $-10(2)$ \\
\hline $\mathrm{O}(4)$ & $27(2)$ & $44(3)$ & $36(3)$ & $2(2)$ & $1(2)$ & $9(2)$ \\
\hline$C(47)$ & $38(4)$ & $49(5)$ & $35(4)$ & $3(4)$ & $-2(4)$ & $-10(4)$ \\
\hline $\mathrm{F}(1)$ & $65(3)$ & $53(3)$ & $63(3)$ & $6(2)$ & $-19(2)$ & $-34(2)$ \\
\hline $\mathrm{F}(2)$ & $45(3)$ & $40(3)$ & $84(3)$ & $28(2)$ & $0(2)$ & $7(2)$ \\
\hline $\mathrm{F}(3)$ & $47(3)$ & $88(4)$ & $38(2)$ & $3(2)$ & $22(2)$ & $-14(2)$ \\
\hline $\operatorname{Pd}\left(1^{\prime}\right)$ & $18(1)$ & $19(1)$ & $16(1)$ & $3(1)$ & $2(1)$ & $0(1)$ \\
\hline $\mathrm{P}\left(1^{\prime}\right)$ & $18(1)$ & $14(1)$ & $18(1)$ & $1(1)$ & $2(1)$ & $1(1)$ \\
\hline $\mathrm{P}\left(2^{\prime}\right)$ & $16(1)$ & $18(1)$ & $17(1)$ & $2(1)$ & $2(1)$ & $-1(1)$ \\
\hline $\mathrm{O}\left(1^{\prime}\right)$ & $18(2)$ & $15(2)$ & $20(2)$ & $-2(2)$ & $5(2)$ & $1(2)$ \\
\hline$C\left(1^{\prime}\right)$ & $19(3)$ & $21(3)$ & $15(3)$ & $-5(2)$ & $8(2)$ & $-8(3)$ \\
\hline $\mathrm{C}\left(2^{\prime}\right)$ & $23(3)$ & $32(4)$ & $24(3)$ & $-3(3)$ & $1(3)$ & $3(3)$ \\
\hline$C\left(3^{\prime}\right)$ & $20(3)$ & $48(5)$ & $33(4)$ & $-18(3)$ & $7(3)$ & $2(3)$ \\
\hline$C\left(4^{\prime}\right)$ & $27(4)$ & $41(5)$ & $30(4)$ & $-2(3)$ & $9(3)$ & $-10(3)$ \\
\hline
\end{tabular}




\begin{tabular}{|c|c|c|c|c|c|c|}
\hline$C\left(5^{\prime}\right)$ & $48(4)$ & $30(4)$ & $29(4)$ & $2(3)$ & $10(3)$ & $-24(4)$ \\
\hline$C\left(6^{\prime}\right)$ & $34(4)$ & $23(4)$ & $21(3)$ & $-4(3)$ & $-1(3)$ & $-7(3)$ \\
\hline$C\left(7^{\prime}\right)$ & $12(3)$ & $13(3)$ & $30(3)$ & $1(3)$ & $3(3)$ & $1(2)$ \\
\hline$C\left(8^{\prime}\right)$ & $25(3)$ & $29(4)$ & $29(3)$ & $2(3)$ & $6(3)$ & $5(3)$ \\
\hline $\mathrm{C}\left(9^{\prime}\right)$ & $40(4)$ & $22(4)$ & $48(4)$ & $15(3)$ & $13(4)$ & $11(3)$ \\
\hline$C\left(10^{\prime}\right)$ & $27(3)$ & $16(4)$ & $54(5)$ & $-1(3)$ & $11(3)$ & $-3(3)$ \\
\hline$C\left(11^{\prime}\right)$ & $28(4)$ & $23(4)$ & $48(4)$ & $-8(3)$ & $-1(3)$ & $3(3)$ \\
\hline$C\left(12^{\prime}\right)$ & $30(4)$ & $25(4)$ & $31(4)$ & $-1(3)$ & $1(3)$ & $1(3)$ \\
\hline$C\left(13^{\prime}\right)$ & $15(3)$ & $9(3)$ & $23(3)$ & $1(2)$ & $-1(2)$ & $3(2)$ \\
\hline$C\left(14^{\prime}\right)$ & $13(3)$ & $24(4)$ & $23(3)$ & $0(3)$ & $3(2)$ & $6(3)$ \\
\hline$C\left(15^{\prime}\right)$ & $10(3)$ & $33(4)$ & $20(3)$ & $-1(3)$ & $-2(2)$ & $-3(3)$ \\
\hline$C\left(16^{\prime}\right)$ & $19(3)$ & $20(3)$ & $18(3)$ & $2(2)$ & $-3(3)$ & $-1(3)$ \\
\hline$C\left(17^{\prime}\right)$ & $15(3)$ & $10(3)$ & $21(3)$ & $6(2)$ & $2(2)$ & $1(2)$ \\
\hline$C\left(18^{\prime}\right)$ & $16(3)$ & $10(3)$ & $22(3)$ & $5(2)$ & $0(2)$ & $0(2)$ \\
\hline$C\left(19^{\prime}\right)$ & $12(3)$ & $29(4)$ & $18(3)$ & $-6(3)$ & $-1(2)$ & $-2(3)$ \\
\hline$C\left(20^{\prime}\right)$ & $21(3)$ & $38(4)$ & $40(4)$ & $-16(3)$ & $1(3)$ & $-1(3)$ \\
\hline$C\left(21^{\prime}\right)$ & $24(3)$ & $64(5)$ & $19(3)$ & $15(3)$ & $4(3)$ & $-5(3)$ \\
\hline$C\left(22^{\prime}\right)$ & $16(3)$ & $17(3)$ & $18(3)$ & $2(2)$ & $-1(2)$ & $-2(2)$ \\
\hline$C\left(23^{\prime}\right)$ & $23(3)$ & $25(4)$ & $22(3)$ & $-7(3)$ & $7(3)$ & $-6(3)$ \\
\hline$C\left(24^{\prime}\right)$ & $15(3)$ & $19(3)$ & $25(3)$ & $-1(3)$ & $5(3)$ & $3(2)$ \\
\hline$C\left(25^{\prime}\right)$ & $13(3)$ & $25(4)$ & $19(3)$ & $3(3)$ & $-1(2)$ & $-2(3)$ \\
\hline$C\left(26^{\prime}\right)$ & $16(3)$ & $14(3)$ & $16(3)$ & $3(2)$ & $3(2)$ & $-3(2)$ \\
\hline$C\left(27^{\prime}\right)$ & $12(3)$ & $14(3)$ & $19(3)$ & $2(2)$ & $6(2)$ & $-4(2)$ \\
\hline $\mathrm{C}\left(28^{\prime}\right)$ & $22(3)$ & $22(4)$ & $14(3)$ & $2(2)$ & $6(2)$ & $4(3)$ \\
\hline$C\left(29^{\prime}\right)$ & $24(3)$ & $31(4)$ & $23(3)$ & $2(3)$ & $5(3)$ & $0(3)$ \\
\hline$C\left(30^{\prime}\right)$ & $22(3)$ & $52(5)$ & $24(3)$ & $-6(3)$ & $1(3)$ & $-13(3)$ \\
\hline$C\left(31^{\prime}\right)$ & $20(3)$ & $60(5)$ & $16(3)$ & $-6(3)$ & $-2(3)$ & $2(4)$ \\
\hline$C\left(32^{\prime}\right)$ & $26(3)$ & $40(4)$ & $18(3)$ & $-1(3)$ & $2(3)$ & $16(3)$ \\
\hline$C\left(33^{\prime}\right)$ & $25(3)$ & $32(4)$ & $25(3)$ & $5(3)$ & $6(3)$ & $0(3)$ \\
\hline$C\left(34^{\prime}\right)$ & $12(3)$ & $25(4)$ & $16(3)$ & $1(3)$ & $1(2)$ & $-1(3)$ \\
\hline$C\left(35^{\prime}\right)$ & $13(3)$ & $27(4)$ & $24(3)$ & $-4(3)$ & $6(3)$ & $2(3)$ \\
\hline$C\left(36^{\prime}\right)$ & $24(3)$ & $23(4)$ & $24(3)$ & $9(3)$ & $5(3)$ & $1(3)$ \\
\hline$C\left(37^{\prime}\right)$ & $23(3)$ & $17(4)$ & $39(4)$ & $10(3)$ & $3(3)$ & $6(3)$ \\
\hline$C\left(38^{\prime}\right)$ & $21(3)$ & $21(4)$ & $38(4)$ & $-4(3)$ & $7(3)$ & $-4(3)$ \\
\hline$C\left(39^{\prime}\right)$ & $29(3)$ & $25(4)$ & $21(3)$ & $5(3)$ & $11(3)$ & $-1(3)$ \\
\hline$C\left(40^{\prime}\right)$ & $22(3)$ & $45(4)$ & $20(3)$ & $13(3)$ & $-4(3)$ & $5(3)$ \\
\hline
\end{tabular}




\begin{tabular}{lcccccc}
$\mathrm{C}\left(41^{\prime}\right)$ & $28(3)$ & $35(4)$ & $13(3)$ & $6(3)$ & $1(3)$ & $3(3)$ \\
$\mathrm{C}\left(42^{\prime}\right)$ & $37(4)$ & $36(4)$ & $19(3)$ & $8(3)$ & $2(3)$ & $-2(3)$ \\
$\mathrm{C}\left(43^{\prime}\right)$ & $31(4)$ & $54(5)$ & $35(4)$ & $-3(4)$ & $12(3)$ & $8(4)$ \\
$\mathrm{C}\left(44^{\prime}\right)$ & $34(4)$ & $52(5)$ & $20(3)$ & $0(3)$ & $6(3)$ & $-5(4)$ \\
$\mathrm{C}\left(45^{\prime}\right)$ & $49(5)$ & $48(5)$ & $24(4)$ & $5(3)$ & $10(3)$ & $-15(4)$ \\
$\mathrm{C}\left(46^{\prime}\right)$ & $48(4)$ & $33(4)$ & $19(3)$ & $2(3)$ & $7(3)$ & $2(3)$ \\
$\mathrm{S}\left(1^{\prime}\right)$ & $78(2)$ & $60(2)$ & $47(1)$ & $-7(1)$ & $21(1)$ & $-9(1)$ \\
$\mathrm{O}\left(2^{\prime}\right)$ & $196(8)$ & $111(6)$ & $44(4)$ & $-36(4)$ & $57(5)$ & $-55(6)$ \\
$\mathrm{O}\left(3^{\prime}\right)$ & $145(6)$ & $66(4)$ & $63(4)$ & $-29(3)$ & $25(4)$ & $-60(4)$ \\
$\mathrm{O}\left(4^{\prime}\right)$ & $149(6)$ & $46(4)$ & $61(4)$ & $22(3)$ & $26(4)$ & $26(4)$ \\
$\mathrm{C}\left(47^{\prime}\right)$ & $139(12)$ & $320(20)$ & $63(7)$ & $109(11)$ & $-74(8)$ & $-184(15)$ \\
$\mathrm{F}\left(1^{\prime}\right)$ & $206(9)$ & $158(8)$ & $192(9)$ & $63(7)$ & $-81(7)$ & $34(7)$ \\
$\mathrm{F}\left(2^{\prime}\right)$ & $107(5)$ & $118(6)$ & $167(7)$ & $-39(5)$ & $45(5)$ & $8(5)$ \\
$\mathrm{F}\left(3^{\prime}\right)$ & $124(6)$ & $160(8)$ & $124(6)$ & $20(5)$ & $-23(5)$ & $-63(6)$ \\
& & & & & & \\
\hline
\end{tabular}


Table S10. Hydrogen coordinates $\left(x 1^{4}\right)$ and isotropic displacement parameters $\left(\AA^{2} \times 10\right.$ 3 ) for 6.

\begin{tabular}{|c|c|c|c|c|}
\hline & $\mathrm{x}$ & $\mathrm{y}$ & $\mathrm{z}$ & $\mathrm{U}(\mathrm{eq})$ \\
\hline $\mathrm{H}(2 \mathrm{~A})$ & 4600 & 4607 & 4014 & 39 \\
\hline $\mathrm{H}(3 \mathrm{~A})$ & 5642 & 4881 & 3637 & 53 \\
\hline $\mathrm{H}(4 \mathrm{~A})$ & 5806 & 6074 & 3322 & 60 \\
\hline $\mathrm{H}(5 \mathrm{~A})$ & 4948 & 6995 & 3405 & 46 \\
\hline $\mathrm{H}(6 \mathrm{~A})$ & 3910 & 6733 & 3799 & 36 \\
\hline $\mathrm{H}(8 \mathrm{~A})$ & 3769 & 4609 & 5269 & 30 \\
\hline $\mathrm{H}(9 \mathrm{~A})$ & 3808 & 3332 & 5446 & 34 \\
\hline $\mathrm{H}(10 \mathrm{~A})$ & 3500 & 2486 & 4758 & 39 \\
\hline $\mathrm{H}(11 \mathrm{~A})$ & 3083 & 2905 & 3897 & 48 \\
\hline $\mathrm{H}(12 \mathrm{~A})$ & 3050 & 4165 & 3708 & 43 \\
\hline $\mathrm{H}(14 \mathrm{~A})$ & 4759 & 6218 & 4884 & 25 \\
\hline $\mathrm{H}(15 \mathrm{~A})$ & 5008 & 6853 & 5679 & 23 \\
\hline $\mathrm{H}(16 \mathrm{~A})$ & 4122 & 7018 & 6218 & 27 \\
\hline $\mathrm{H}(20 \mathrm{~A})$ & 2353 & 5894 & 6691 & 51 \\
\hline $\mathrm{H}(20 \mathrm{~B})$ & 3233 & 5888 & 6701 & 51 \\
\hline $\mathrm{H}(20 \mathrm{C})$ & 2699 & 5396 & 6273 & 51 \\
\hline $\mathrm{H}(21 \mathrm{~A})$ & 2693 & 7668 & 6141 & 59 \\
\hline $\mathrm{H}(21 \mathrm{~B})$ & 3207 & 7266 & 6630 & 59 \\
\hline $\mathrm{H}(21 \mathrm{C})$ & 2323 & 7281 & 6598 & 59 \\
\hline $\mathrm{H}(23 \mathrm{~A})$ & 1255 & 7027 & 6144 & 27 \\
\hline $\mathrm{H}(24 \mathrm{~A})$ & 153 & 6896 & 5557 & 30 \\
\hline $\mathrm{H}(25 \mathrm{~A})$ & 116 & 6307 & 4741 & 25 \\
\hline $\mathrm{H}(29 \mathrm{~A})$ & -124 & 4689 & 4030 & 37 \\
\hline $\mathrm{H}(30 \mathrm{~A})$ & -1338 & 4958 & 3620 & 56 \\
\hline $\mathrm{H}(31 \mathrm{~A})$ & -1653 & 6100 & 3237 & 50 \\
\hline $\mathrm{H}(32 \mathrm{~A})$ & -740 & 6987 & 3225 & 38 \\
\hline $\mathrm{H}(33 \mathrm{~A})$ & 479 & 6759 & 3627 & 28 \\
\hline $\mathrm{H}(35 \mathrm{~A})$ & 1398 & 4656 & 5181 & 46 \\
\hline $\mathrm{H}(36 \mathrm{~A})$ & 1521 & 3396 & 5365 & 65 \\
\hline $\mathrm{H}(37 \mathrm{~A})$ & 1488 & 2550 & 4674 & 66 \\
\hline
\end{tabular}




\begin{tabular}{|c|c|c|c|c|}
\hline $\mathrm{H}(38 \mathrm{~A})$ & 1327 & 2975 & 3793 & 66 \\
\hline $\mathrm{H}(39 \mathrm{~A})$ & 1222 & 4242 & 3610 & 51 \\
\hline $\mathrm{H}(40 \mathrm{~A})$ & 1103 & 5444 & 2925 & 34 \\
\hline $\mathrm{H}(40 \mathrm{~B})$ & 1170 & 6353 & 2979 & 34 \\
\hline $\mathrm{H}(42 \mathrm{~A})$ & 2300 & 6970 & 2783 & 44 \\
\hline $\mathrm{H}(43 \mathrm{~A})$ & 3480 & 6897 & 2531 & 55 \\
\hline $\mathrm{H}(44 \mathrm{~A})$ & 4020 & 5744 & 2445 & 58 \\
\hline $\mathrm{H}(45 \mathrm{~A})$ & 3458 & 4657 & 2680 & 50 \\
\hline $\mathrm{H}(46 \mathrm{~A})$ & 2273 & 4727 & 2908 & 42 \\
\hline $\mathrm{H}\left(2^{\prime} \mathrm{A}\right)$ & 4640 & -263 & 4059 & 32 \\
\hline $\mathrm{H}\left(3^{\prime} \mathrm{A}\right)$ & 5694 & 180 & 3742 & 40 \\
\hline $\mathrm{H}\left(4^{\prime} \mathrm{A}\right)$ & 5748 & 1405 & 3468 & 38 \\
\hline $\mathrm{H}\left(5^{\prime} \mathrm{A}\right)$ & 4769 & 2207 & 3515 & 42 \\
\hline $\mathrm{H}\left(6^{\prime} \mathrm{A}\right)$ & 3711 & 1784 & 3822 & 32 \\
\hline $\mathrm{H}\left(8^{\prime} \mathrm{A}\right)$ & 3632 & -497 & 5224 & 33 \\
\hline $\mathrm{H}\left(9^{\prime} \mathrm{A}\right)$ & 3718 & -1783 & 5319 & 43 \\
\hline $\mathrm{H}(10 \mathrm{~B})$ & 3464 & -2544 & 4580 & 38 \\
\hline $\mathrm{H}(11 \mathrm{~B})$ & 3157 & -2036 & 3723 & 41 \\
\hline $\mathrm{H}(12 \mathrm{~B})$ & 3071 & -750 & 3625 & 35 \\
\hline $\mathrm{H}(14 \mathrm{~B})$ & 4713 & 1093 & 4902 & 24 \\
\hline $\mathrm{H}(15 \mathrm{~B})$ & 5032 & 1732 & 5699 & 26 \\
\hline $\mathrm{H}(16 \mathrm{~B})$ & 4126 & 2050 & 6201 & 24 \\
\hline $\mathrm{H}(20 \mathrm{D})$ & 2709 & 2847 & 5995 & 50 \\
\hline $\mathrm{H}(20 \mathrm{E})$ & 3235 & 2543 & 6517 & 50 \\
\hline $\mathrm{H}(20 \mathrm{~F})$ & 2357 & 2605 & 6503 & 50 \\
\hline $\mathrm{H}(21 \mathrm{D})$ & 2660 & 632 & 6412 & 54 \\
\hline $\mathrm{H}(21 \mathrm{E})$ & 2338 & 1250 & 6762 & 54 \\
\hline $\mathrm{H}(21 \mathrm{~F})$ & 3214 & 1197 & 6765 & 54 \\
\hline $\mathrm{H}(23 \mathrm{~B})$ & 1257 & 2215 & 6131 & 27 \\
\hline $\mathrm{H}(24 \mathrm{~B})$ & 129 & 2032 & 5590 & 23 \\
\hline $\mathrm{H}(25 \mathrm{~B})$ & 55 & 1335 & 4800 & 23 \\
\hline H(29B) & -55 & -475 & 4013 & 31 \\
\hline $\mathrm{H}(30 \mathrm{~B})$ & -1281 & -356 & 3592 & 40 \\
\hline $\mathrm{H}(31 \mathrm{~B})$ & -1750 & 783 & 3264 & 39 \\
\hline $\mathrm{H}(32 \mathrm{~B})$ & -971 & 1824 & 3359 & 34 \\
\hline H(33B) & 254 & 1714 & 3782 & 33 \\
\hline
\end{tabular}




\begin{tabular}{lrrrr}
$\mathrm{H}(35 \mathrm{~B})$ & 1302 & -275 & 5287 & 25 \\
$\mathrm{H}(36 \mathrm{~B})$ & 1533 & -1519 & 5536 & 28 \\
$\mathrm{H}(37 \mathrm{~B})$ & 1611 & -2407 & 4873 & 32 \\
$\mathrm{H}(38 \mathrm{~B})$ & 1511 & -2040 & 3986 & 32 \\
$\mathrm{H}(39 B)$ & 1366 & -797 & 3758 & 29 \\
$\mathrm{H}(40 \mathrm{C})$ & 994 & 557 & 2959 & 36 \\
$\mathrm{H}(40 \mathrm{D})$ & 948 & 1432 & 3138 & 36 \\
$\mathrm{H}(42 \mathrm{~B})$ & 2277 & 64 & 2900 & 37 \\
$\mathrm{H}(43 \mathrm{~B})$ & 3426 & 296 & 2634 & 47 \\
$\mathrm{H}(44 \mathrm{~B})$ & 3788 & 1502 & 2493 & 42 \\
$\mathrm{H}(45 \mathrm{~B})$ & 3043 & 2493 & 2651 & 48 \\
$\mathrm{H}(46 \mathrm{~B})$ & 1917 & 2300 & 2925 & 40 \\
& & & & \\
\hline
\end{tabular}




\section{Experimental Procedure for the X-Ray Diffraction of 7}

\section{$\underline{\text { Data Collection }}$}

A yellow blade crystal of $\mathrm{C}_{44} \mathrm{H}_{35} \mathrm{~F}_{3} \mathrm{O}_{4} \mathrm{P}_{2} \mathrm{PdS} \cdot \mathrm{CH}_{2} \mathrm{Cl}_{2}, \mathrm{C}_{45} \mathrm{H}_{37} \mathrm{Cl}_{2} \mathrm{O}_{4} \mathrm{P}_{2} \mathrm{PdS}$, having approximate dimensions of $0.20 \times 0.10 \times 0.08 \mathrm{~mm}^{3}$ was mounted with epoxy cement on the tip of a fine glass fiber. All measurements were made on a Nonius KappaCCD diffractometer with graphite monochromated Mo-K $\alpha$ radiation.

Cell constants and an orientation matrix for data collection corresponded to a triclinic cell with dimensions:

$$
\begin{array}{ll}
\mathrm{a}=11.451(2) \AA & \alpha=83.76(3)^{\mathrm{o}} \\
\mathrm{b}=14.314(3) \AA & \beta=67.63(3)^{\mathrm{o}} \\
\mathrm{c}=14.412(3) \AA & \gamma=70.75(3)^{\mathrm{o}} \\
\mathrm{V}=2062.0(7) \AA^{3} &
\end{array}
$$

For $Z=2$ and F.W. $=970.05$, the calculated density is $1.562 \mathrm{~g} / \mathrm{cm}^{3}$. Based on a statistical analysis of intensity distribution, and the successful solution and refinement of the structure, the space group was determined to be $P-1(\# 2)$.

The data were collected at a temperature of $173(2) \mathrm{K}$ to a maximum $2 \theta$ value of $56.52^{\circ}$. Seven omega scans consisting of $37,37,34,29,24,29$, and 7 data frames, respectively, were collected with a frame width of $2.0^{\circ}$ and a detector-to-crystal distance, Dx, of $35.0 \mathrm{~mm}$. Each frame was exposed twice (for the purpose of de-zingering) for a total of $70 \mathrm{~s}$. The data frames were processed and scaled using the DENZO software package. ${ }^{1}$

\section{$\underline{\text { Data Reduction }}$}

A total of 17206 reflections were collected of which 10129 were unique and observed $\left(\mathrm{R}_{\text {int }}=0.0583\right)$. The linear absorption coefficient, $\mu$, for Mo-K $\alpha$ radiation is $7.66 \mathrm{~cm}^{-1}$, and no absorption correction was applied. The data were corrected for Lorentz and polarization effects.

\section{$\underline{\text { Structure Solution and Refinement }}$}

The structure was solved by direct methods and expanded using Fourier techniques ${ }^{2}$. The non-hydrogen atoms were refined anisotropically, and hydrogen atoms with exceptions noted were treated as idealized contributions. The final cycle of full-matrix least-squares refinement ${ }^{3}$ on $\mathrm{F}$ was based on 10129 observed reflections (I > 2.00б(I)) and 554 variable parameters and converged with unweighted and weighted agreement factors of:

$$
\begin{gathered}
\mathrm{R}=\Sigma\|\mathrm{Fo}|-| \mathrm{Fc}\| / \Sigma|\mathrm{Fo}|=0.0510 \\
\mathrm{R}_{\mathrm{W}}=\left\{\Sigma\left[\mathrm{W}\left(\mathrm{F}_{\mathrm{o}}{ }^{2}-\mathrm{F}_{\mathrm{c}}{ }^{2}\right)^{2}\right] / \Sigma\left[\mathrm{w}\left(\mathrm{F}_{\mathrm{o}}{ }^{2}\right)^{2}\right]\right\}^{1 / 2}=0.0843
\end{gathered}
$$


The maximum and minimum peaks on the final difference Fourier map corresponded to 0.607 and $-0.942 \mathrm{e}^{-/} \AA^{3}$ respectively.

\section{REFERENCES}

(1) Z. Otwinowski and W. Minor, "Processing of X-Ray Diffraction Data Collected in Oscillation Mode," Methods in Enzymology, vol. 276: Macromolecular Crystallography, part A, 307-326, 1997, C.W. Carter, Jr. \& R.M. Sweet, Eds., Academic Press.

(2) Acta Cryst. A46 (1990) 467-473

(3) Least Squares function minimized:

$$
\Sigma w\left(\mathrm{~F}_{\mathrm{o}}^{2}-\mathrm{F}_{\mathrm{c}}{ }^{2}\right)^{2}
$$




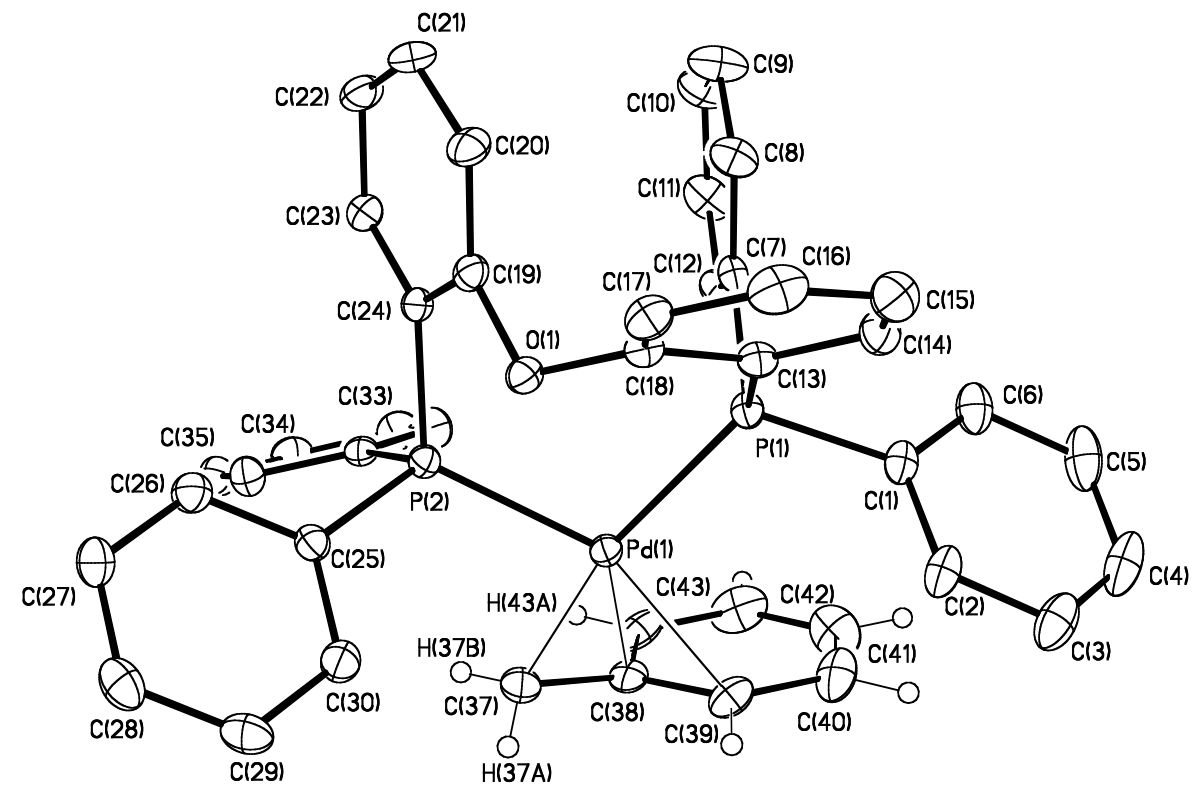

Figure S7. ORTEP diagram of 7 
Table S11. Crystal data and structure refinement for 7.

Empirical formula

Formula weight

Temperature

Wavelength

Crystal system

Space group

Unit cell dimensions

Volume

Z

Density (calculated)

Absorption coefficient

$\mathrm{F}(000)$

Crystal size

Theta range for data collection

Index ranges

Reflections collected

Independent reflections

Completeness to theta $=28.26^{\circ}$

Absorption correction

Max. and min. transmission

Refinement method

Data / restraints / parameters

Goodness-of-fit on $\mathrm{F}^{2}$

Final $\mathrm{R}$ indices [I $>2 \operatorname{sigma}(\mathrm{I})]$

$\mathrm{R}$ indices (all data)

Largest diff. peak and hole
$\mathrm{C}_{45} \mathrm{H}_{37} \mathrm{Cl}_{2} \mathrm{~F}_{3} \mathrm{O}_{4} \mathrm{P}_{2} \mathrm{Pd} \mathrm{S}$

970.05

173(2) K

$0.71073 \AA$

Triclinic

P-1

$\mathrm{a}=11.451(2) \AA$

$\alpha=83.76(3)^{\circ}$.

$\mathrm{b}=14.314(3) \AA$

$\beta=67.63(3)^{\circ}$.

$\mathrm{c}=14.412(3) \AA$

$\gamma=70.75(3)^{\circ}$.
2

$1.562 \mathrm{~g} / \mathrm{cm}^{3}$

$7.66 \mathrm{~cm}^{-1}$

984

$0.20 \times 0.10 \times 0.08 \mathrm{~mm}^{3}$

2.87 to $28.26^{\circ}$.

$-15<=\mathrm{h}<=15,-19<=\mathrm{k}<=19,-19<=\mathrm{l}<=19$

17206

$10129[\mathrm{R}(\mathrm{int})=0.0583]$

$99.2 \%$

None

0.9413 and 0.8619

Full-matrix least-squares on $\mathrm{F}^{2}$

10129 / 0 / 554

1.002

$\mathrm{R} 1=0.0510, \mathrm{wR} 2=0.0843$

$\mathrm{R} 1=0.1087, \mathrm{wR} 2=0.0976$

0.607 and -0.942 e. $\AA^{-3}$ 
Table S12. Atomic coordinates ( $x \mathbf{1 0}^{4}$ ) and equivalent isotropic displacement parameters $\left(\AA^{2} \mathbf{x} \mathbf{1 0}^{3}\right)$ for $7 . \mathrm{U}(\mathrm{eq})$ is defined as one third of the trace of the orthogonalized Uij tensor.

\begin{tabular}{|c|c|c|c|c|}
\hline & $\mathrm{x}$ & $\mathrm{y}$ & z & $\mathrm{U}(\mathrm{eq})$ \\
\hline $\operatorname{Pd}(1)$ & $5316(1)$ & $3121(1)$ & $2060(1)$ & $24(1)$ \\
\hline $\mathrm{P}(1)$ & $5574(1)$ & $1777(1)$ & $3138(1)$ & $24(1)$ \\
\hline $\mathrm{P}(2)$ & $6300(1)$ & 2411(1) & $504(1)$ & $24(1)$ \\
\hline $\mathrm{S}(1)$ & $8304(1)$ & $4244(1)$ & $2668(1)$ & $40(1)$ \\
\hline $\mathrm{O}(1)$ & $8175(2)$ & $1127(2)$ & $1318(2)$ & $26(1)$ \\
\hline $\mathrm{O}(2)$ & $9019(3)$ & $4742(2)$ & $1853(2)$ & $68(1)$ \\
\hline $\mathrm{O}(3)$ & $8215(3)$ & $4480(2)$ & $3631(2)$ & $67(1)$ \\
\hline $\mathrm{O}(4)$ & $7132(3)$ & $4170(3)$ & $2603(2)$ & $75(1)$ \\
\hline $\mathrm{F}(1)$ & $8922(3)$ & $2400(2)$ & $3200(2)$ & $78(1)$ \\
\hline $\mathrm{F}(2)$ & $9601(3)$ & $2595(2)$ & $1604(2)$ & $73(1)$ \\
\hline $\mathrm{F}(3)$ & $10586(2)$ & $2914(2)$ & $2463(2)$ & $68(1)$ \\
\hline $\mathrm{C}(1)$ & $4766(3)$ & $2182(2)$ & $4442(2)$ & $29(1)$ \\
\hline$C(2)$ & $5251(4)$ & $2812(3)$ & $4769(3)$ & $38(1)$ \\
\hline$C(3)$ & $4627(4)$ & $3210(3)$ & $5732(3)$ & $49(1)$ \\
\hline$C(4)$ & $3518(4)$ & 2994(3) & $6378(3)$ & $56(1)$ \\
\hline$C(5)$ & $3042(4)$ & $2357(3)$ & $6080(3)$ & $53(1)$ \\
\hline$C(6)$ & $3667(3)$ & 1952(3) & $5108(3)$ & $40(1)$ \\
\hline$C(7)$ & $4737(3)$ & $950(2)$ & $3000(2)$ & $25(1)$ \\
\hline$C(8)$ & $5302(3)$ & $-74(3)$ & 2931(3) & $38(1)$ \\
\hline$C(9)$ & $4632(4)$ & $-660(3)$ & $2782(3)$ & $51(1)$ \\
\hline$C(10)$ & $3400(4)$ & $-231(3)$ & $2720(3)$ & $50(1)$ \\
\hline$C(11)$ & $2807(4)$ & 784(3) & $2824(3)$ & $45(1)$ \\
\hline$C(12)$ & $3488(3)$ & $1366(3)$ & $2944(2)$ & $34(1)$ \\
\hline$C(13)$ & $7215(3)$ & $977(2)$ & $3112(2)$ & $25(1)$ \\
\hline$C(14)$ & $7420(3)$ & 593(3) & $3994(3)$ & $34(1)$ \\
\hline$C(15)$ & $8657(4)$ & $48(3)$ & $3981(3)$ & $38(1)$ \\
\hline$C(16)$ & $9733(4)$ & $-123(3)$ & $3094(3)$ & $41(1)$ \\
\hline$C(17)$ & $9571(3)$ & $237(2)$ & $2209(3)$ & $32(1)$ \\
\hline$C(18)$ & $8321(3)$ & $764(2)$ & $2215(2)$ & $25(1)$ \\
\hline C(19) & $7795(3)$ & $548(2)$ & $845(2)$ & $26(1)$ \\
\hline
\end{tabular}




\begin{tabular}{|c|c|c|c|c|}
\hline$C(20)$ & $8308(3)$ & $-472(2)$ & $787(2)$ & $32(1)$ \\
\hline $\mathrm{C}(21)$ & $7889(4)$ & $-986(3)$ & 281(3) & $39(1)$ \\
\hline$C(22)$ & $6993(4)$ & $-495(3)$ & $-167(3)$ & $36(1)$ \\
\hline $\mathrm{C}(23)$ & $6499(3)$ & $522(2)$ & $-112(2)$ & $31(1)$ \\
\hline$C(24)$ & $6889(3)$ & $1068(2)$ & $393(2)$ & $25(1)$ \\
\hline$C(25)$ & $7737(3)$ & $2757(2)$ & $-321(2)$ & $26(1)$ \\
\hline$C(26)$ & $8476(3)$ & $2336(3)$ & $-1279(2)$ & $34(1)$ \\
\hline $\mathrm{C}(27)$ & $9530(3)$ & $2644(3)$ & $-1918(3)$ & $40(1)$ \\
\hline $\mathrm{C}(28)$ & $9874(4)$ & $3349(3)$ & $-1606(3)$ & $43(1)$ \\
\hline C(29) & $9188(3)$ & $3750(3)$ & $-655(3)$ & $41(1)$ \\
\hline$C(30)$ & $8118(3)$ & $3462(3)$ & $-11(3)$ & $34(1)$ \\
\hline $\mathrm{C}(31)$ & $5086(3)$ & $2770(2)$ & $-106(2)$ & $25(1)$ \\
\hline$C(32)$ & $3776(3)$ & $2835(3)$ & $488(3)$ & $35(1)$ \\
\hline$C(33)$ & $2770(4)$ & $3200(3)$ & $106(3)$ & $40(1)$ \\
\hline$C(34)$ & $3054(4)$ & $3500(3)$ & $-876(3)$ & $38(1)$ \\
\hline$C(35)$ & $4346(4)$ & $3415(3)$ & $-1466(3)$ & $41(1)$ \\
\hline$C(36)$ & $5357(3)$ & $3068(3)$ & $-1099(2)$ & $34(1)$ \\
\hline $\mathrm{C}(37)$ & $4749(5)$ & $4497(3)$ & 1401(3) & $40(1)$ \\
\hline$C(38)$ & $3674(3)$ & $4603(2)$ & $2341(3)$ & $31(1)$ \\
\hline C(39) & $3977(4)$ & $4500(3)$ & $3217(3)$ & $37(1)$ \\
\hline $\mathrm{C}(40)$ & $2982(4)$ & $4473(3)$ & $4158(3)$ & $51(1)$ \\
\hline $\mathrm{C}(41)$ & $1772(4)$ & $4498(3)$ & $4219(4)$ & $61(1)$ \\
\hline $\mathrm{C}(42)$ & $1469(4)$ & $4564(3)$ & $3369(4)$ & $57(1)$ \\
\hline $\mathrm{C}(43)$ & 2391(4) & $4618(3)$ & $2447(3)$ & $45(1)$ \\
\hline$C(44)$ & $9403(4)$ & $2980(3)$ & $2469(3)$ & $44(1)$ \\
\hline$C(45)$ & $1414(5)$ & $-3004(4)$ & $4474(4)$ & $112(2)$ \\
\hline $\mathrm{Cl}(1)$ & $2271(3)$ & $-2198(2)$ & $3932(2)$ & $76(1)$ \\
\hline $\mathrm{Cl}(2)$ & $-303(13)$ & $-2516(9)$ & $4847(10)$ & $68(2)$ \\
\hline $\mathrm{Cl}\left(1^{\prime}\right)$ & $2397(5)$ & $-2630(4)$ & $3553(4)$ & $67(1)$ \\
\hline $\mathrm{Cl}\left(2^{\prime}\right)$ & $-190(30)$ & $-2460(20)$ & $4970(20)$ & $108(7)$ \\
\hline
\end{tabular}


Table S13. Bond lengths $[\AA]]$ and angles $\left[^{\circ}\right]$ for 7 .

\begin{tabular}{|c|c|c|c|}
\hline $\operatorname{Pd}(1)-C(37)$ & $2.102(4)$ & $C(14)-C(15)$ & $1.368(5)$ \\
\hline $\mathrm{Pd}(1)-\mathrm{P}(2)$ & $2.2690(12)$ & $C(15)-C(16)$ & $1.372(5)$ \\
\hline $\operatorname{Pd}(1)-C(38)$ & $2.275(3)$ & $C(16)-C(17)$ & $1.378(5)$ \\
\hline $\mathrm{Pd}(1)-\mathrm{P}(1)$ & $2.3523(12)$ & $\mathrm{C}(17)-\mathrm{C}(18)$ & $1.378(4)$ \\
\hline $\operatorname{Pd}(1)-C(39)$ & $2.408(4)$ & $C(19)-C(20)$ & $1.380(4)$ \\
\hline $\mathrm{P}(1)-\mathrm{C}(1)$ & $1.812(3)$ & $C(19)-C(24)$ & $1.398(4)$ \\
\hline $\mathrm{P}(1)-\mathrm{C}(7)$ & $1.817(3)$ & $C(20)-C(21)$ & $1.384(5)$ \\
\hline $\mathrm{P}(1)-\mathrm{C}(13)$ & $1.839(3)$ & $\mathrm{C}(21)-\mathrm{C}(22)$ & $1.376(5)$ \\
\hline$P(2)-C(25)$ & $1.813(3)$ & $C(22)-C(23)$ & $1.375(5)$ \\
\hline$P(2)-C(24)$ & $1.818(3)$ & $C(23)-C(24)$ & $1.394(4)$ \\
\hline$P(2)-C(31)$ & $1.825(3)$ & $\mathrm{C}(25)-\mathrm{C}(30)$ & $1.393(4)$ \\
\hline $\mathrm{S}(1)-\mathrm{O}(4)$ & $1.419(3)$ & $C(25)-C(26)$ & $1.394(4)$ \\
\hline $\mathrm{S}(1)-\mathrm{O}(3)$ & $1.422(3)$ & $C(26)-C(27)$ & $1.384(4)$ \\
\hline $\mathrm{S}(1)-\mathrm{O}(2)$ & $1.424(3)$ & $\mathrm{C}(27)-\mathrm{C}(28)$ & $1.369(5)$ \\
\hline$S(1)-C(44)$ & $1.810(4)$ & $\mathrm{C}(28)-\mathrm{C}(29)$ & $1.371(5)$ \\
\hline $\mathrm{O}(1)-\mathrm{C}(18)$ & $1.389(4)$ & $C(29)-C(30)$ & $1.385(5)$ \\
\hline $\mathrm{O}(1)-\mathrm{C}(19)$ & $1.389(4)$ & $C(31)-C(32)$ & $1.393(4)$ \\
\hline $\mathrm{F}(1)-\mathrm{C}(44)$ & $1.330(4)$ & $\mathrm{C}(31)-\mathrm{C}(36)$ & $1.394(4)$ \\
\hline$F(2)-C(44)$ & $1.327(4)$ & $C(32)-C(33)$ & $1.388(5)$ \\
\hline$F(3)-C(44)$ & $1.324(4)$ & $\mathrm{C}(33)-\mathrm{C}(34)$ & $1.380(5)$ \\
\hline$C(1)-C(6)$ & $1.378(5)$ & $C(34)-C(35)$ & $1.369(5)$ \\
\hline$C(1)-C(2)$ & $1.402(5)$ & $C(35)-C(36)$ & $1.377(5)$ \\
\hline$C(2)-C(3)$ & $1.382(5)$ & $\mathrm{C}(37)-\mathrm{H}(37 \mathrm{~B})$ & $0.83(4)$ \\
\hline$C(3)-C(4)$ & $1.368(6)$ & $\mathrm{C}(37)-\mathrm{H}(37 \mathrm{~A})$ & $0.96(4)$ \\
\hline$C(4)-C(5)$ & $1.380(6)$ & $\mathrm{C}(37)-\mathrm{C}(38)$ & $1.423(5)$ \\
\hline$C(5)-C(6)$ & $1.396(5)$ & $\mathrm{C}(38)-\mathrm{C}(43)$ & $1.411(5)$ \\
\hline $\mathrm{C}(7)-\mathrm{C}(12)$ & $1.386(4)$ & $\mathrm{C}(38)-\mathrm{C}(39)$ & $1.412(5)$ \\
\hline$C(7)-C(8)$ & $1.391(5)$ & $\mathrm{C}(39)-\mathrm{H}(39 \mathrm{~A})$ & $0.95(3)$ \\
\hline $\mathrm{C}(8)-\mathrm{C}(9)$ & $1.388(5)$ & $C(39)-C(40)$ & $1.408(5)$ \\
\hline $\mathrm{C}(9)-\mathrm{C}(10)$ & $1.374(5)$ & $\mathrm{C}(40)-\mathrm{C}(41)$ & $1.343(6)$ \\
\hline$C(10)-C(11)$ & $1.384(5)$ & $\mathrm{C}(41)-\mathrm{C}(42)$ & $1.381(6)$ \\
\hline $\mathrm{C}(11)-\mathrm{C}(12)$ & $1.377(5)$ & $\mathrm{C}(42)-\mathrm{C}(43)$ & $1.361(6)$ \\
\hline$C(13)-C(18)$ & $1.398(4)$ & $\mathrm{C}(45)-\mathrm{Cl}\left(1^{\prime}\right)$ & $1.556(7)$ \\
\hline$C(13)-C(14)$ & $1.401(4)$ & $\mathrm{C}(45)-\mathrm{Cl}\left(2^{\prime}\right)$ & $1.64(3)$ \\
\hline
\end{tabular}




\begin{tabular}{|c|c|c|c|}
\hline $\mathrm{C}(45)-\mathrm{Cl}(1)$ & $1.694(6)$ & $C(4)-C(3)-C(2)$ & $119.9(4)$ \\
\hline \multirow[t]{2}{*}{$\mathrm{C}(45)-\mathrm{Cl}(2)$} & $1.736(14)$ & $C(3)-C(4)-C(5)$ & $120.2(4)$ \\
\hline & & $C(4)-C(5)-C(6)$ & $120.1(4)$ \\
\hline $\mathrm{C}(37)-\mathrm{Pd}(1)-\mathrm{P}(2)$ & $88.62(12)$ & $C(1)-C(6)-C(5)$ & $120.3(4)$ \\
\hline $\mathrm{C}(37)-\mathrm{Pd}(1)-\mathrm{C}(38)$ & $37.68(14)$ & $C(12)-C(7)-C(8)$ & $118.9(3)$ \\
\hline $\mathrm{P}(2)-\mathrm{Pd}(1)-\mathrm{C}(38)$ & 119.61(10) & $\mathrm{C}(12)-\mathrm{C}(7)-\mathrm{P}(1)$ & $118.1(3)$ \\
\hline $\mathrm{C}(37)-\mathrm{Pd}(1)-\mathrm{P}(1)$ & $166.72(11)$ & $\mathrm{C}(8)-\mathrm{C}(7)-\mathrm{P}(1)$ & $123.0(3)$ \\
\hline $\mathrm{P}(2)-\mathrm{Pd}(1)-\mathrm{P}(1)$ & $104.08(4)$ & $C(9)-C(8)-C(7)$ & $120.0(3)$ \\
\hline $\mathrm{C}(38)-\mathrm{Pd}(1)-\mathrm{P}(1)$ & $129.45(9)$ & $\mathrm{C}(10)-\mathrm{C}(9)-\mathrm{C}(8)$ & $120.1(4)$ \\
\hline $\mathrm{C}(37)-\mathrm{Pd}(1)-\mathrm{C}(39)$ & $64.55(15)$ & $C(9)-C(10)-C(11)$ & $120.4(4)$ \\
\hline $\mathrm{P}(2)-\mathrm{Pd}(1)-\mathrm{C}(39)$ & 152.99(10) & $C(12)-C(11)-C(10)$ & $119.3(3)$ \\
\hline $\mathrm{C}(38)-\mathrm{Pd}(1)-\mathrm{C}(39)$ & $34.95(12)$ & $C(11)-C(12)-C(7)$ & $121.2(3)$ \\
\hline $\mathrm{P}(1)-\mathrm{Pd}(1)-\mathrm{C}(39)$ & $102.51(10)$ & $\mathrm{C}(18)-\mathrm{C}(13)-\mathrm{C}(14)$ & $117.4(3)$ \\
\hline $\mathrm{C}(1)-\mathrm{P}(1)-\mathrm{C}(7)$ & $105.30(15)$ & $\mathrm{C}(18)-\mathrm{C}(13)-\mathrm{P}(1)$ & $121.2(2)$ \\
\hline$C(1)-P(1)-C(13)$ & $101.14(15)$ & $\mathrm{C}(14)-\mathrm{C}(13)-\mathrm{P}(1)$ & $121.4(2)$ \\
\hline$C(7)-P(1)-C(13)$ & $105.84(15)$ & $C(15)-C(14)-C(13)$ & $121.4(3)$ \\
\hline $\mathrm{C}(1)-\mathrm{P}(1)-\mathrm{Pd}(1)$ & $111.21(11)$ & $C(14)-C(15)-C(16)$ & $120.0(3)$ \\
\hline $\mathrm{C}(7)-\mathrm{P}(1)-\mathrm{Pd}(1)$ & $109.51(11)$ & $C(15)-C(16)-C(17)$ & $120.3(3)$ \\
\hline$C(13)-P(1)-P d(1)$ & $122.39(11)$ & $C(18)-C(17)-C(16)$ & $119.8(3)$ \\
\hline$C(25)-P(2)-C(24)$ & $103.20(15)$ & $\mathrm{C}(17)-\mathrm{C}(18)-\mathrm{O}(1)$ & $119.1(3)$ \\
\hline $\mathrm{C}(25)-\mathrm{P}(2)-\mathrm{C}(31)$ & $107.08(15)$ & $C(17)-C(18)-C(13)$ & $121.0(3)$ \\
\hline $\mathrm{C}(24)-\mathrm{P}(2)-\mathrm{C}(31)$ & $103.17(15)$ & $\mathrm{O}(1)-\mathrm{C}(18)-\mathrm{C}(13)$ & $119.8(3)$ \\
\hline$C(25)-P(2)-P d(1)$ & $115.08(11)$ & $\mathrm{C}(20)-\mathrm{C}(19)-\mathrm{O}(1)$ & $122.7(3)$ \\
\hline$C(24)-P(2)-P d(1)$ & $118.60(11)$ & $C(20)-C(19)-C(24)$ & $121.8(3)$ \\
\hline $\mathrm{C}(31)-\mathrm{P}(2)-\mathrm{Pd}(1)$ & $108.58(11)$ & $\mathrm{O}(1)-\mathrm{C}(19)-\mathrm{C}(24)$ & $115.5(3)$ \\
\hline $\mathrm{O}(4)-\mathrm{S}(1)-\mathrm{O}(3)$ & $116.48(19)$ & $C(19)-C(20)-C(21)$ & $118.6(3)$ \\
\hline $\mathrm{O}(4)-\mathrm{S}(1)-\mathrm{O}(2)$ & 114.30(19) & $C(22)-C(21)-C(20)$ & $121.0(3)$ \\
\hline $\mathrm{O}(3)-\mathrm{S}(1)-\mathrm{O}(2)$ & $115.20(19)$ & $C(23)-C(22)-C(21)$ & $119.8(3)$ \\
\hline $\mathrm{O}(4)-\mathrm{S}(1)-\mathrm{C}(44)$ & 103.29(19) & $\mathrm{C}(22)-\mathrm{C}(23)-\mathrm{C}(24)$ & $121.1(3)$ \\
\hline $\mathrm{O}(3)-\mathrm{S}(1)-\mathrm{C}(44)$ & $102.22(18)$ & $C(23)-C(24)-C(19)$ & $117.7(3)$ \\
\hline $\mathrm{O}(2)-\mathrm{S}(1)-\mathrm{C}(44)$ & $102.50(19)$ & $\mathrm{C}(23)-\mathrm{C}(24)-\mathrm{P}(2)$ & $124.5(3)$ \\
\hline$C(18)-O(1)-C(19)$ & $115.6(2)$ & $C(19)-C(24)-P(2)$ & $117.8(2)$ \\
\hline$C(6)-C(1)-C(2)$ & $118.6(3)$ & $C(30)-C(25)-C(26)$ & $118.5(3)$ \\
\hline $\mathrm{C}(6)-\mathrm{C}(1)-\mathrm{P}(1)$ & $123.9(3)$ & $\mathrm{C}(30)-\mathrm{C}(25)-\mathrm{P}(2)$ & $120.5(2)$ \\
\hline $\mathrm{C}(2)-\mathrm{C}(1)-\mathrm{P}(1)$ & $117.5(3)$ & $\mathrm{C}(26)-\mathrm{C}(25)-\mathrm{P}(2)$ & $121.0(2)$ \\
\hline$C(3)-C(2)-C(1)$ & $120.9(4)$ & $C(27)-C(26)-C(25)$ & $120.3(3)$ \\
\hline
\end{tabular}




$\begin{array}{lclc}\mathrm{C}(28)-\mathrm{C}(27)-\mathrm{C}(26) & 120.2(3) & \mathrm{F}(2)-\mathrm{C}(44)-\mathrm{F}(1) & 107.8(3) \\ \mathrm{C}(27)-\mathrm{C}(28)-\mathrm{C}(29) & 120.4(3) & \mathrm{F}(3)-\mathrm{C}(44)-\mathrm{S}(1) & 111.6(3) \\ \mathrm{C}(28)-\mathrm{C}(29)-\mathrm{C}(30) & 120.1(3) & \mathrm{F}(2)-\mathrm{C}(44)-\mathrm{S}(1) & 112.5(3) \\ \mathrm{C}(29)-\mathrm{C}(30)-\mathrm{C}(25) & 120.4(3) & \mathrm{F}(1)-\mathrm{C}(44)-\mathrm{S}(1) & 111.3(3) \\ \mathrm{C}(32)-\mathrm{C}(31)-\mathrm{C}(36) & 117.9(3) & \mathrm{Cl}\left(1^{\prime}\right)-\mathrm{C}(45)-\mathrm{Cl}\left(2^{\prime}\right) & 125.2(11) \\ \mathrm{C}(32)-\mathrm{C}(31)-\mathrm{P}(2) & 117.5(2) & \mathrm{Cl}\left(1^{\prime}\right)-\mathrm{C}(45)-\mathrm{Cl}(1) & 28.45(19) \\ \mathrm{C}(36)-\mathrm{C}(31)-\mathrm{P}(2) & 124.3(3) & \mathrm{Cl}\left(2^{\prime}\right)-\mathrm{C}(45)-\mathrm{Cl}(1) & 112.7(10) \\ \mathrm{C}(33)-\mathrm{C}(32)-\mathrm{C}(31) & 121.0(3) & \mathrm{Cl}\left(1^{\prime}\right)-\mathrm{C}(45)-\mathrm{Cl}(2) & 123.4(6) \\ \mathrm{C}(34)-\mathrm{C}(33)-\mathrm{C}(32) & 120.3(3) & \mathrm{Cl}\left(2^{\prime}\right)-\mathrm{C}(45)-\mathrm{Cl}(2) & \\ \mathrm{C}(35)-\mathrm{C}(34)-\mathrm{C}(33) & 118.6(3) & \mathrm{Cl}(1)-\mathrm{C}(45)-\mathrm{Cl}(2) & 115.6(5) \\ \mathrm{C}(34)-\mathrm{C}(35)-\mathrm{C}(36) & 122.0(3) & & \\ \mathrm{C}(35)-\mathrm{C}(36)-\mathrm{C}(31) & 120.1(3) & & \\ \mathrm{H}(37 \mathrm{~B})-\mathrm{C}(37)-\mathrm{H}(37 \mathrm{~A}) & 111(3) & & \\ \mathrm{H}(37 \mathrm{~B})-\mathrm{C}(37)-\mathrm{C}(38) & 120(3) & & \\ \mathrm{H}(37 \mathrm{~A})-\mathrm{C}(37)-\mathrm{C}(38) & 119(2) & & \\ \mathrm{H}(37 \mathrm{~B})-\mathrm{C}(37)-\mathrm{Pd}(1) & 122(3) & & \\ \mathrm{H}(37 \mathrm{~A})-\mathrm{C}(37)-\mathrm{Pd}(1) & 103(2) & & \\ \mathrm{C}(38)-\mathrm{C}(37)-\mathrm{Pd}(1) & 77.8(2) & & \\ \mathrm{C}(43)-\mathrm{C}(38)-\mathrm{C}(39) & 117.9(3) & & \\ \mathrm{C}(43)-\mathrm{C}(38)-\mathrm{C}(37) & 123.6(4) & & \\ \mathrm{C}(39)-\mathrm{C}(38)-\mathrm{C}(37) & 117.4(4) & \\ \mathrm{C}(43)-\mathrm{C}(38)-\mathrm{Pd}(1) & 118.5(2) & \\ \mathrm{C}(39)-\mathrm{C}(38)-\mathrm{Pd}(1) & 77.7(2) & & \\ \mathrm{C}(37)-\mathrm{C}(38)-\mathrm{Pd}(1) & 64.6(2) & \\ \mathrm{H}(39 \mathrm{~A})-\mathrm{C}(39)-\mathrm{C}(40) & 117.9(17) & \\ \mathrm{H}(39 \mathrm{~A})-\mathrm{C}(39)-\mathrm{C}(38) & 121.0(17) & \\ \mathrm{C}(40)-\mathrm{C}(39)-\mathrm{C}(38) & 119.4(4) & \\ \mathrm{H}(39 \mathrm{~A})-\mathrm{C}(39)-\mathrm{Pd}(1) & 90.3(17) & \\ \mathrm{C}(40)-\mathrm{C}(39)-\mathrm{Pd}(1) & 125.7(3) & & \\ \mathrm{C}(38)-\mathrm{C}(39)-\mathrm{Pd}(1) & 67.35(19) & \\ \mathrm{C}(41)-\mathrm{C}(40)-\mathrm{C}(39) & 120.3(4) & \\ \mathrm{C}(40)-\mathrm{C}(41)-\mathrm{C}(42) & 121.2(4) & \\ \mathrm{C}(43)-\mathrm{C}(42)-\mathrm{C}(41) & 120.4(4) & \\ \mathrm{C}(42)-\mathrm{C}(43)-\mathrm{C}(38) & 120.7(4) & \\ \mathrm{F}(3)-\mathrm{C}(44)-\mathrm{F}(2) & 107.0(3) & \\ \mathrm{F}(3)-\mathrm{C}(44)-\mathrm{F}(1) & 106.3(3) & & \\ & & & \\ \end{array}$


Table S14. Anisotropic displacement parameters $\left(\AA^{2} \times 10^{3}\right)$ for 7 . The anisotropic displacement factor exponent takes the form: $-2 \pi^{2}\left[\mathrm{~h}^{2} \mathrm{a}^{* 2} \mathrm{U}^{11}+\ldots+2 \mathrm{hk} \mathrm{a} \mathrm{b}^{*} \mathrm{U}^{12}\right]$

\begin{tabular}{|c|c|c|c|c|c|c|}
\hline & $\mathrm{U}^{11}$ & $\mathrm{U}^{22}$ & $\mathrm{U}^{33}$ & $\mathrm{U}^{23}$ & $\mathrm{U}^{13}$ & $\mathrm{U}^{12}$ \\
\hline $\operatorname{Pd}(1)$ & $26(1)$ & $20(1)$ & $23(1)$ & $-1(1)$ & $-7(1)$ & $-4(1)$ \\
\hline $\mathrm{P}(1)$ & $22(1)$ & $25(1)$ & $22(1)$ & $1(1)$ & $-7(1)$ & $-4(1)$ \\
\hline $\mathrm{P}(2)$ & $25(1)$ & $23(1)$ & $22(1)$ & $0(1)$ & $-7(1)$ & $-6(1)$ \\
\hline $\mathrm{S}(1)$ & $38(1)$ & $38(1)$ & $45(1)$ & $1(1)$ & $-21(1)$ & $-9(1)$ \\
\hline $\mathrm{O}(1)$ & $26(1)$ & $24(1)$ & $26(1)$ & $-2(1)$ & $-10(1)$ & $-6(1)$ \\
\hline $\mathrm{O}(2)$ & $80(2)$ & $54(2)$ & $76(2)$ & $33(2)$ & $-32(2)$ & $-32(2)$ \\
\hline $\mathrm{O}(3)$ & $87(2)$ & $49(2)$ & $52(2)$ & $-20(1)$ & $-33(2)$ & $9(2)$ \\
\hline $\mathrm{O}(4)$ & $33(2)$ & 111(3) & $83(2)$ & $-7(2)$ & $-22(2)$ & $-23(2)$ \\
\hline $\mathrm{F}(1)$ & 111(2) & $46(2)$ & $76(2)$ & $16(1)$ & $-28(2)$ & $-36(2)$ \\
\hline $\mathrm{F}(2)$ & $97(2)$ & $59(2)$ & $64(2)$ & $-24(1)$ & $-32(2)$ & $-13(2)$ \\
\hline $\mathrm{F}(3)$ & $49(2)$ & $60(2)$ & $86(2)$ & $-4(1)$ & $-33(1)$ & $2(1)$ \\
\hline$C(1)$ & $24(2)$ & $32(2)$ & $24(2)$ & $-1(2)$ & $-11(2)$ & $2(2)$ \\
\hline$C(2)$ & $34(2)$ & $49(2)$ & $27(2)$ & $-8(2)$ & $-14(2)$ & $-2(2)$ \\
\hline$C(3)$ & $45(3)$ & $57(3)$ & $43(2)$ & $-13(2)$ & $-26(2)$ & $4(2)$ \\
\hline$C(4)$ & $46(3)$ & $77(3)$ & $35(2)$ & $-16(2)$ & $-21(2)$ & $5(2)$ \\
\hline$C(5)$ & $28(2)$ & $77(3)$ & $29(2)$ & $2(2)$ & $-2(2)$ & $4(2)$ \\
\hline$C(6)$ & $31(2)$ & $51(3)$ & $30(2)$ & $3(2)$ & $-11(2)$ & $-3(2)$ \\
\hline$C(7)$ & $25(2)$ & $29(2)$ & $20(2)$ & $3(1)$ & $-7(1)$ & $-8(2)$ \\
\hline$C(8)$ & $31(2)$ & $33(2)$ & $50(2)$ & $7(2)$ & $-18(2)$ & $-9(2)$ \\
\hline$C(9)$ & $51(3)$ & $30(2)$ & $82(3)$ & $7(2)$ & $-34(2)$ & $-16(2)$ \\
\hline$C(10)$ & $50(3)$ & $45(3)$ & $70(3)$ & $11(2)$ & $-31(2)$ & $-28(2)$ \\
\hline $\mathrm{C}(11)$ & $33(2)$ & $51(3)$ & $58(3)$ & $14(2)$ & $-24(2)$ & $-19(2)$ \\
\hline$C(12)$ & $29(2)$ & $30(2)$ & $38(2)$ & $6(2)$ & $-14(2)$ & $-6(2)$ \\
\hline$C(13)$ & $22(2)$ & $21(2)$ & $31(2)$ & $0(1)$ & $-12(2)$ & $-3(1)$ \\
\hline$C(14)$ & $34(2)$ & $33(2)$ & $31(2)$ & $1(2)$ & $-13(2)$ & $-6(2)$ \\
\hline$C(15)$ & $39(2)$ & $36(2)$ & $41(2)$ & $3(2)$ & $-23(2)$ & $-5(2)$ \\
\hline$C(16)$ & $31(2)$ & $33(2)$ & $58(3)$ & $-6(2)$ & $-25(2)$ & $1(2)$ \\
\hline$C(17)$ & $24(2)$ & $29(2)$ & $37(2)$ & $-7(2)$ & $-9(2)$ & $-1(2)$ \\
\hline $\mathrm{C}(18)$ & $25(2)$ & $22(2)$ & $29(2)$ & $-1(2)$ & $-12(2)$ & $-7(2)$ \\
\hline C(19) & $23(2)$ & $29(2)$ & $24(2)$ & $-4(2)$ & $-4(2)$ & $-10(2)$ \\
\hline
\end{tabular}




$\begin{array}{lllllll}\mathrm{C}(20) & 32(2) & 25(2) & 35(2) & -5(2) & -13(2) & 1(2) \\ \mathrm{C}(21) & 49(2) & 19(2) & 44(2) & -6(2) & -14(2) & -6(2) \\ \mathrm{C}(22) & 45(2) & 30(2) & 38(2) & -6(2) & -18(2) & -12(2) \\ \mathrm{C}(23) & 36(2) & 30(2) & 27(2) & -1(2) & -12(2) & -12(2) \\ \mathrm{C}(24) & 24(2) & 24(2) & 22(2) & -1(1) & -2(1) & -8(2) \\ \mathrm{C}(25) & 26(2) & 25(2) & 25(2) & 3(2) & -9(2) & -8(2) \\ \mathrm{C}(26) & 32(2) & 33(2) & 34(2) & -2(2) & -9(2) & -11(2) \\ \mathrm{C}(27) & 33(2) & 46(2) & 31(2) & -2(2) & 0(2) & -13(2) \\ \mathrm{C}(28) & 32(2) & 50(3) & 42(2) & 8(2) & -6(2) & -17(2) \\ \mathrm{C}(29) & 38(2) & 39(2) & 53(3) & 4(2) & -17(2) & -22(2) \\ \mathrm{C}(30) & 35(2) & 37(2) & 30(2) & -1(2) & -11(2) & -13(2) \\ \mathrm{C}(31) & 29(2) & 17(2) & 27(2) & -1(1) & -9(2) & -3(2) \\ \mathrm{C}(32) & 35(2) & 39(2) & 31(2) & 6(2) & -13(2) & -11(2) \\ \mathrm{C}(33) & 32(2) & 44(2) & 47(2) & -4(2) & -15(2) & -12(2) \\ \mathrm{C}(34) & 41(2) & 38(2) & 47(2) & 2(2) & -28(2) & -13(2) \\ \mathrm{C}(35) & 59(3) & 44(2) & 31(2) & 10(2) & -25(2) & -25(2) \\ \mathrm{C}(36) & 34(2) & 39(2) & 29(2) & 3(2) & -12(2) & -13(2) \\ \mathrm{C}(37) & 55(3) & 20(2) & 38(3) & 2(2) & -14(2) & -6(2) \\ \mathrm{C}(38) & 35(2) & 15(2) & 36(2) & -3(2) & -14(2) & 3(2) \\ \mathrm{C}(39) & 27(2) & 34(2) & 44(2) & -14(2) & -13(2) & 3(2) \\ \mathrm{C}(40) & 46(3) & 45(3) & 38(2) & -11(2) & -14(2) & 16(2) \\ \mathrm{C}(41) & 33(3) & 56(3) & 63(3) & 1(2) & 1(2) & 2(2) \\ \mathrm{C}(42) & 23(2) & 54(3) & 83(4) & -22(3) & -13(2) & 3(2) \\ \mathrm{C}(43) & 42(2) & 35(2) & 56(3) & -19(2) & -30(2) & 9(2) \\ \mathrm{C}(44) & 54(3) & 39(2) & 46(3) & 1(2) & -20(2) & -22(2) \\ \mathrm{C}(45) & 55(3) & 110(5) & 114(5) & 70(4) & -10(3) & 0(3) \\ \mathrm{Cl}(1) & 65(2) & 82(2) & 103(3) & 37(2) & -48(2) & -43(2) \\ \mathrm{Cl}(2) & 45(3) & 86(3) & 65(2) & 23(2) & -15(2) & -23(3) \\ \mathrm{Cl}(1) & 51(2) & 74(4) & 67(3) & 20(2) & -15(2) & -23(3) \\ \mathrm{Cl}\left(2^{\prime}\right) & 54(6) & 139(13) & 103(11) & 64(9) & -22(6) & -21(7)\end{array}$


Table S15. Hydrogen coordinates $\left(\begin{array}{ll}x & 10^{4}\end{array}\right)$ and isotropic displacement parameters $\left(\AA^{2} \times 10^{3}\right)$ for 7 .

\begin{tabular}{|c|c|c|c|c|}
\hline & $\mathrm{x}$ & $\mathrm{y}$ & z & $\mathrm{U}(\mathrm{eq})$ \\
\hline $\mathrm{H}(2 \mathrm{~A})$ & 6017 & 2968 & 4322 & 46 \\
\hline $\mathrm{H}(3 \mathrm{~A})$ & 4968 & 3634 & 5945 & 59 \\
\hline $\mathrm{H}(4 \mathrm{~A})$ & 3075 & 3283 & 7033 & 67 \\
\hline $\mathrm{H}(5 \mathrm{~A})$ & 2286 & 2193 & 6537 & 63 \\
\hline $\mathrm{H}(6 \mathrm{~A})$ & 3334 & 1516 & 4905 & 48 \\
\hline $\mathrm{H}(8 \mathrm{~A})$ & 6146 & -373 & 2985 & 45 \\
\hline $\mathrm{H}(9 \mathrm{~A})$ & 5026 & -1358 & 2722 & 61 \\
\hline $\mathrm{H}(10 \mathrm{~A})$ & 2952 & -635 & 2606 & 60 \\
\hline $\mathrm{H}(11 \mathrm{~A})$ & 1938 & 1077 & 2811 & 54 \\
\hline $\mathrm{H}(12 \mathrm{~A})$ & 3096 & 2065 & 2990 & 40 \\
\hline $\mathrm{H}(14 \mathrm{~A})$ & 6684 & 713 & 4614 & 40 \\
\hline $\mathrm{H}(15 \mathrm{~A})$ & 8772 & -213 & 4587 & 46 \\
\hline $\mathrm{H}(16 \mathrm{~A})$ & 10594 & -491 & 3089 & 49 \\
\hline $\mathrm{H}(17 \mathrm{~A})$ & 10319 & 121 & 1597 & 39 \\
\hline $\mathrm{H}(20 \mathrm{~A})$ & 8934 & -814 & 1088 & 39 \\
\hline $\mathrm{H}(21 \mathrm{~A})$ & 8227 & -1688 & 242 & 47 \\
\hline $\mathrm{H}(22 \mathrm{~A})$ & 6716 & -857 & -512 & 43 \\
\hline $\mathrm{H}(23 \mathrm{~A})$ & 5881 & 857 & -424 & 37 \\
\hline $\mathrm{H}(26 \mathrm{~A})$ & 8256 & 1834 & -1494 & 40 \\
\hline $\mathrm{H}(27 \mathrm{~A})$ & 10015 & 2365 & -2576 & 48 \\
\hline $\mathrm{H}(28 \mathrm{~A})$ & 10594 & 3561 & -2050 & 52 \\
\hline $\mathrm{H}(29 \mathrm{~A})$ & 9447 & 4227 & -438 & 49 \\
\hline $\mathrm{H}(30 \mathrm{~A})$ & 7640 & 3746 & 645 & 41 \\
\hline $\mathrm{H}(32 \mathrm{~A})$ & 3568 & 2627 & 1164 & 42 \\
\hline $\mathrm{H}(33 \mathrm{~A})$ & 1881 & 3243 & 524 & 48 \\
\hline $\mathrm{H}(34 \mathrm{~A})$ & 2367 & 3760 & -1138 & 46 \\
\hline $\mathrm{H}(35 \mathrm{~A})$ & 4552 & 3601 & -2149 & 49 \\
\hline $\mathrm{H}(36 \mathrm{~A})$ & 6241 & 3032 & -1524 & 40 \\
\hline
\end{tabular}




\begin{tabular}{lllll}
$\mathrm{H}(37 \mathrm{~A})$ & $5450(40)$ & $4760(30)$ & $1330(30)$ & $47(12)$ \\
$\mathrm{H}(37 \mathrm{~B})$ & $4600(40)$ & $4540(30)$ & $870(30)$ & $42(12)$ \\
$\mathrm{H}(39 \mathrm{~A})$ & $4760(30)$ & $4610(20)$ & $3210(20)$ & $18(8)$ \\
$\mathrm{H}(40 \mathrm{~A})$ & 3166 & 4436 & 4752 & 61 \\
$\mathrm{H}(41 \mathrm{~A})$ & 1113 & 4469 & 4858 & 73 \\
$\mathrm{H}(42 \mathrm{~A})$ & 610 & 4572 & 3428 & 69 \\
$\mathrm{H}(43 \mathrm{~A})$ & 2169 & 4667 & 1868 & 54 \\
$\mathrm{H}(45 \mathrm{~A})$ & 1632 & -3281 & 5069 & 135 \\
$\mathrm{H}(45 \mathrm{~B})$ & 1727 & -3559 & 3995 & 135 \\
& & & & \\
\hline
\end{tabular}




\section{Experimental Procedure for the X-Ray Diffraction of 9}

\section{$\underline{\text { Data Collection }}$}

A yellow block crystal of $\mathrm{C}_{42} \mathrm{H}_{35} \mathrm{~F}_{3} \mathrm{FeO}_{3} \mathrm{P}_{2} \mathrm{PdS} \cdot 0.5 \mathrm{CH}_{2} \mathrm{Cl}_{2}, \mathrm{C}_{42.50} \mathrm{H}_{36} \mathrm{ClF}_{3} \mathrm{FeO}_{3} \mathrm{P}_{2} \mathrm{PdS}$, having approximate dimensions of $0.15 \times 0.10 \times 0.10 \mathrm{~mm}^{3}$ was mounted with epoxy cement on the tip of a fine glass fiber. All measurements were made on a Nonius KappaCCD diffractometer with graphite monochromated Mo-K $\alpha$ radiation.

Cell constants and an orientation matrix for data collection corresponded to a triclinic cell with dimensions:

$$
\begin{array}{ll}
\mathrm{a}=10.426(2) \AA & \alpha=63.91(3)^{\mathrm{o}} \\
\mathrm{b}=20.056(4) \AA & \beta=81.69(3)^{\mathrm{o}} \\
\mathrm{c}=21.214(4) \AA & \gamma=77.45(3)^{\mathrm{o}} \\
\mathrm{V}=3882.8(13) \AA^{3} &
\end{array}
$$

For $\mathrm{Z}=4$ and F.W. $=943.41$, the calculated density is $1.614 \mathrm{~g} / \mathrm{cm}^{3}$. Based on a statistical analysis of intensity distribution, and the successful solution and refinement of the structure, the space group was determined to be $P-1$ (\#2).

The data were collected at a temperature of $173(2) \mathrm{K}$ to a maximum $2 \theta$ value of $56.60^{\circ}$. Seven omega scans consisting of 44, 44, 40, 35, 26, 35, and 9 data frames, respectively, were collected with a frame width of $1.7^{\circ}$ and a detector-to-crystal distance, Dx, of $35.0 \mathrm{~mm}$. Each frame was exposed twice (for the purpose of de-zingering) for a total of $68 \mathrm{~s}$. The data frames were processed and scaled using the DENZO software package. ${ }^{1}$

\section{Data Reduction}

A total of 32734 reflections were collected of which 19196 were unique and observed $\left(\mathrm{R}_{\text {int }}=0.0651\right)$. The linear absorption coefficient, $\mu$, for Mo-K $\alpha$ radiation is $10.96 \mathrm{~cm}^{-1}$, and no absorption correction was applied. The data were corrected for Lorentz and polarization effects.

\section{$\underline{\text { Structure Solution and Refinement }}$}

The structure was solved by direct methods and expanded using Fourier techniques ${ }^{2}$. The non-hydrogen atoms were refined anisotropically, and hydrogen atoms, with exceptions noted, were treated as idealized contributions. The final cycle of full-matrix least-squares refinement ${ }^{3}$

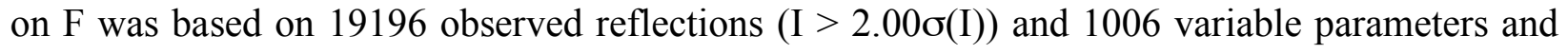
converged with unweighted and weighted agreement factors of:

$$
\begin{gathered}
\mathrm{R}=\Sigma\|\mathrm{Fo}|-| \mathrm{Fc}\| / \Sigma|\mathrm{Fo}|=0.0501 \\
\mathrm{R}_{\mathrm{W}}=\left\{\Sigma\left[\mathrm{w}\left(\mathrm{F}_{\mathrm{o}}{ }^{2}-\mathrm{F}_{\mathrm{c}}{ }^{2}\right)^{2}\right] / \Sigma\left[\mathrm{w}\left(\mathrm{F}_{\mathrm{o}}{ }^{2}\right)^{2}\right]\right\}^{1 / 2}=0.0762
\end{gathered}
$$

The maximum and minimum peaks on the final difference Fourier map corresponded to 0.594 and $-0.800 \mathrm{e}^{-} / \AA^{3}$ respectively. 


\section{REFERENCES}

(1) Z. Otwinowski and W. Minor, "Processing of X-Ray Diffraction Data Collected in Oscillation Mode," Methods in Enzymology, vol. 276: Macromolecular Crystallography, part A, 307-326, 1997, C.W. Carter, Jr. \& R.M. Sweet, Eds., Academic Press.

(2) Acta Cryst. A46 (1990) 467-473

(3) Least Squares function minimized:

$$
\Sigma w\left(\mathrm{~F}_{\mathrm{o}}^{2}-\mathrm{F}_{\mathrm{c}}^{2}\right)^{2}
$$




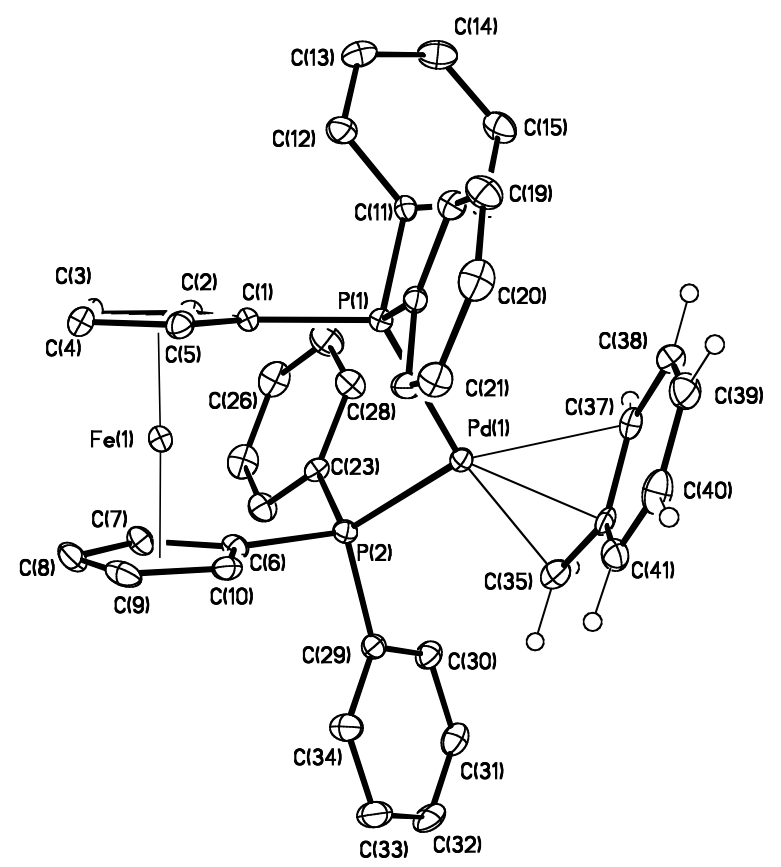

Figure S8. ORTEP diagram of $9\left(1^{\text {st }}\right.$ independent molecule)

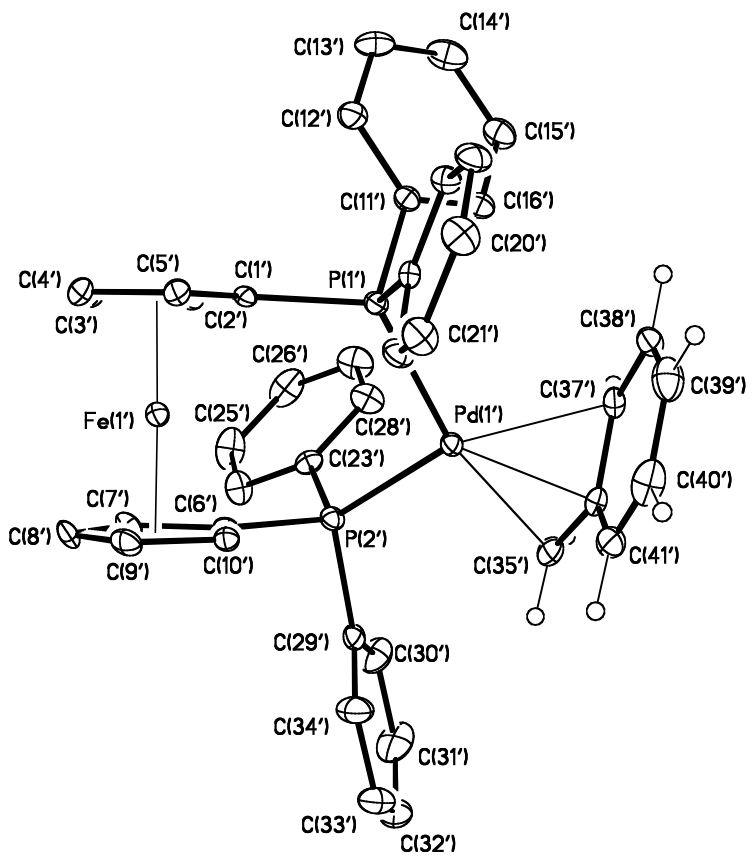

Figure S9. ORTEP diagram of 9 ( $2^{\text {st }}$ independent molecule) 
Table S16. Crystal data and structure refinement for 9.

Empirical formula

Formula weight

Temperature

Wavelength

Crystal system

Space group

Unit cell dimensions

Volume

Z

Density (calculated)

Absorption coefficient

$\mathrm{F}(000)$

Crystal size

Theta range for data collection

Index ranges

Reflections collected

Independent reflections

Completeness to theta $=28.30^{\circ}$

Absorption correction

Max. and min. transmission

Refinement method

Data / restraints / parameters

Goodness-of-fit on $\mathrm{F}^{2}$

Final $\mathrm{R}$ indices [I $>2 \operatorname{sigma}(\mathrm{I})]$

$\mathrm{R}$ indices (all data)

Largest diff. peak and hole
$\mathrm{C}_{42.50} \mathrm{H}_{36} \mathrm{Cl} \mathrm{F}_{3} \mathrm{Fe} \mathrm{O}_{3} \mathrm{P}_{2} \mathrm{Pd} \mathrm{S}$

943.41

173(2) K

$0.71073 \AA$

Triclinic

P-1

$\mathrm{a}=10.426(2) \AA \quad \alpha=63.91(3)^{\circ}$.

$\mathrm{b}=20.056(4) \AA \quad \beta=81.69(3)^{\circ}$.

$\mathrm{c}=21.214(4) \AA \quad \gamma=77.45(3)^{\circ}$.

$3882.8(13) \AA^{3}$

4

$1.614 \mathrm{~g} / \mathrm{cm}^{3}$

$10.96 \mathrm{~cm}^{-1}$

1908

$0.15 \times 0.10 \times 0.10 \mathrm{~mm}^{3}$

2.00 to $28.30^{\circ}$.

$-13<=\mathrm{h}<=13,-26<=\mathrm{k}<=25,-27<=\mathrm{k}<=28$

32734

$19196[\mathrm{R}(\mathrm{int})=0.0651]$

$99.5 \%$

None

0.8983 and 0.8528

Full-matrix least-squares on $\mathrm{F}^{2}$

19196 / 0 / 1006

0.978

$\mathrm{R} 1=0.0501, \mathrm{wR} 2=0.0762$

$\mathrm{R} 1=0.1224, \mathrm{wR} 2=0.0897$

0.594 and -0.800 e. $\AA^{-3}$ 
Table S17. Atomic coordinates ( $x \mathbf{1 0}^{4}$ ) and equivalent isotropic displacement parameters $\left(\AA^{2} \times 10^{3}\right)$ for 9. U(eq) is defined as one third of the trace of the orthogonalized $U^{i j}$ tensor.

\begin{tabular}{|c|c|c|c|c|}
\hline & $\mathrm{x}$ & $\mathrm{y}$ & $\mathrm{z}$ & $\mathrm{U}(\mathrm{eq})$ \\
\hline $\operatorname{Pd}(1)$ & $88(1)$ & $2492(1)$ & $7277(1)$ & $19(1)$ \\
\hline $\mathrm{Fe}(1)$ & $-2609(1)$ & $2490(1)$ & $8946(1)$ & $21(1)$ \\
\hline $\mathrm{P}(1)$ & $160(1)$ & $1539(1)$ & $8428(1)$ & $19(1)$ \\
\hline $\mathrm{P}(2)$ & $-965(1)$ & $3520(1)$ & $7453(1)$ & $21(1)$ \\
\hline $\mathrm{C}(1)$ & $-992(3)$ & $1673(2)$ & $9105(2)$ & $20(1)$ \\
\hline $\mathrm{C}(2)$ & $-898(3)$ & $2147(2)$ & $9433(2)$ & $22(1)$ \\
\hline $\mathrm{C}(3)$ & $-1962(4)$ & $2105(2)$ & $9935(2)$ & $29(1)$ \\
\hline$C(4)$ & $-2728(4)$ & $1605(2)$ & $9927(2)$ & $29(1)$ \\
\hline $\mathrm{C}(5)$ & $-2125(4)$ & $1339(2)$ & $9419(2)$ & $24(1)$ \\
\hline$C(6)$ & $-2464(3)$ & $3358(2)$ & $7989(2)$ & $22(1)$ \\
\hline$C(7)$ & $-3048(4)$ & $3638(2)$ & $8503(2)$ & $29(1)$ \\
\hline$C(8)$ & $-4216(4)$ & $3334(2)$ & $8793(2)$ & $34(1)$ \\
\hline $\mathrm{C}(9)$ & $-4370(4)$ & $2875(2)$ & $8473(2)$ & $32(1)$ \\
\hline$C(10)$ & $-3305(3)$ & $2885(2)$ & $7972(2)$ & $26(1)$ \\
\hline$C(11)$ & 1707(3) & $1220(2)$ & $8854(2)$ & $19(1)$ \\
\hline$C(12)$ & $1762(4)$ & $852(2)$ & $9585(2)$ & $31(1)$ \\
\hline$C(13)$ & $2940(4)$ & $674(2)$ & $9892(2)$ & $37(1)$ \\
\hline$C(14)$ & $4079(4)$ & $859(2)$ & $9480(2)$ & $39(1)$ \\
\hline$C(15)$ & $4047(4)$ & $1205(2)$ & $8758(2)$ & $34(1)$ \\
\hline$C(16)$ & $2859(4)$ & $1387(2)$ & $8450(2)$ & $28(1)$ \\
\hline$C(17)$ & $-281(3)$ & $726(2)$ & $8401(2)$ & $19(1)$ \\
\hline$C(18)$ & $445(4)$ & $0(2)$ & $8689(2)$ & $25(1)$ \\
\hline$C(19)$ & $61(4)$ & $-586(2)$ & $8623(2)$ & $31(1)$ \\
\hline$C(20)$ & $-1059(4)$ & $-454(2)$ & $8284(2)$ & $33(1)$ \\
\hline$C(21)$ & $-1817(4)$ & $264(2)$ & $8005(2)$ & $28(1)$ \\
\hline$C(22)$ & $-1409(4)$ & $847(2)$ & $8055(2)$ & $26(1)$ \\
\hline$C(23)$ & $10(4)$ & $3839(2)$ & $7876(2)$ & $21(1)$ \\
\hline$C(24)$ & $-413(4)$ & $4513(2)$ & $7939(2)$ & $30(1)$ \\
\hline$C(25)$ & $326(4)$ & $4742(2)$ & $8276(2)$ & $36(1)$ \\
\hline$C(26)$ & $1498(4)$ & $4288(2)$ & $8564(2)$ & $40(1)$ \\
\hline$C(27)$ & $1924(4)$ & $3623(2)$ & $8501(2)$ & $41(1)$ \\
\hline
\end{tabular}




\begin{tabular}{|c|c|c|c|c|}
\hline$C(28)$ & $1200(4)$ & $3401(2)$ & $8152(2)$ & $29(1)$ \\
\hline C(29) & $-1459(4)$ & $4352(2)$ & $6658(2)$ & $24(1)$ \\
\hline $\mathrm{C}(30)$ & $-548(4)$ & $4803(2)$ & $6235(2)$ & $29(1)$ \\
\hline $\mathrm{C}(31)$ & $-907(4)$ & $5404(2)$ & $5596(2)$ & $34(1)$ \\
\hline$C(32)$ & $-2157(4)$ & $5556(2)$ & $5376(2)$ & $35(1)$ \\
\hline $\mathrm{C}(33)$ & $-3064(4)$ & $5113(2)$ & $5788(2)$ & $38(1)$ \\
\hline$C(34)$ & $-2720(4)$ & $4505(2)$ & $6427(2)$ & $32(1)$ \\
\hline$C(35)$ & $73(5)$ & $3093(2)$ & $6183(2)$ & $32(1)$ \\
\hline$C(36)$ & $424(4)$ & $2328(2)$ & $6278(2)$ & $22(1)$ \\
\hline$C(37)$ & $1588(4)$ & $1893(2)$ & $6621(2)$ & $26(1)$ \\
\hline$C(38)$ & $1893(4)$ & $1115(2)$ & $6811(2)$ & $33(1)$ \\
\hline C(39) & $1044(4)$ & $772(2)$ & $6676(2)$ & $37(1)$ \\
\hline$C(40)$ & $-116(4)$ & $1183(2)$ & $6354(2)$ & $36(1)$ \\
\hline$C(41)$ & $-453(4)$ & $1938(2)$ & $6176(2)$ & $31(1)$ \\
\hline $\operatorname{Pd}\left(1^{\prime}\right)$ & $5017(1)$ & $2332(1)$ & $2134(1)$ & $19(1)$ \\
\hline $\operatorname{Fe}\left(1^{\prime}\right)$ & $2560(1)$ & $2553(1)$ & $3792(1)$ & $19(1)$ \\
\hline$P\left(1^{\prime}\right)$ & $5140(1)$ & $1481(1)$ & $3322(1)$ & $19(1)$ \\
\hline$P\left(2^{\prime}\right)$ & $4064(1)$ & $3440(1)$ & $2202(1)$ & $20(1)$ \\
\hline$C\left(1^{\prime}\right)$ & $4164(3)$ & $1725(2)$ & $3989(2)$ & $18(1)$ \\
\hline $\mathrm{C}\left(2^{\prime}\right)$ & $4366(3)$ & $2293(2)$ & $4178(2)$ & $24(1)$ \\
\hline$C\left(3^{\prime}\right)$ & $3377(4)$ & $2340(2)$ & $4704(2)$ & $31(1)$ \\
\hline$C\left(4^{\prime}\right)$ & $2571(4)$ & $1811(2)$ & $4841(2)$ & $30(1)$ \\
\hline$C\left(5^{\prime}\right)$ & $3036(3)$ & $1424(2)$ & $4410(2)$ & $24(1)$ \\
\hline$C\left(6^{\prime}\right)$ & $2612(3)$ & $3332(2)$ & $2784(2)$ & $20(1)$ \\
\hline$C\left(7^{\prime}\right)$ & $2046(3)$ & $3686(2)$ & $3247(2)$ & $24(1)$ \\
\hline$C\left(8^{\prime}\right)$ & $907(3)$ & $3375(2)$ & $3593(2)$ & $26(1)$ \\
\hline $\mathrm{C}\left(9^{\prime}\right)$ & $753(3)$ & $2836(2)$ & $3368(2)$ & $27(1)$ \\
\hline$C\left(10^{\prime}\right)$ & 1794(3) & $2812(2)$ & $2864(2)$ & $20(1)$ \\
\hline$C\left(11^{\prime}\right)$ & $6791(4)$ & $1198(2)$ & $3648(2)$ & $22(1)$ \\
\hline$C\left(12^{\prime}\right)$ & $6989(4)$ & $849(2)$ & $4367(2)$ & $30(1)$ \\
\hline$C\left(13^{\prime}\right)$ & $8244(4)$ & $679(2)$ & $4598(2)$ & $42(1)$ \\
\hline$C\left(14^{\prime}\right)$ & $9302(4)$ & $859(2)$ & $4123(2)$ & $43(1)$ \\
\hline$C\left(15^{\prime}\right)$ & $9125(4)$ & $1200(2)$ & $3410(2)$ & $35(1)$ \\
\hline$C\left(16^{\prime}\right)$ & $7869(3)$ & $1376(2)$ & $3175(2)$ & $26(1)$ \\
\hline$C\left(17^{\prime}\right)$ & $4581(3)$ & $625(2)$ & $3462(2)$ & $18(1)$ \\
\hline$C\left(18^{\prime}\right)$ & $5276(4)$ & $-97(2)$ & $3807(2)$ & $23(1)$ \\
\hline
\end{tabular}




\begin{tabular}{|c|c|c|c|c|}
\hline$C\left(19^{\prime}\right)$ & $4787(4)$ & $-726(2)$ & $3901(2)$ & $31(1)$ \\
\hline$C\left(20^{\prime}\right)$ & $3578(4)$ & $-643(2)$ & $3662(2)$ & $33(1)$ \\
\hline$C\left(21^{\prime}\right)$ & $2860(4)$ & $76(2)$ & $3323(2)$ & $32(1)$ \\
\hline$C\left(22^{\prime}\right)$ & $3375(4)$ & $708(2)$ & $3208(2)$ & $26(1)$ \\
\hline$C\left(23^{\prime}\right)$ & $5207(4)$ & $3813(2)$ & $2469(2)$ & $23(1)$ \\
\hline$C\left(24^{\prime}\right)$ & $4814(4)$ & $4445(2)$ & $2616(2)$ & $33(1)$ \\
\hline$C\left(25^{\prime}\right)$ & $5747(5)$ & $4734(2)$ & $2777(2)$ & $46(1)$ \\
\hline$C\left(26^{\prime}\right)$ & $7060(5)$ & $4411(3)$ & $2788(2)$ & $46(1)$ \\
\hline$C\left(27^{\prime}\right)$ & $7462(4)$ & $3795(2)$ & $2632(2)$ & $43(1)$ \\
\hline$C\left(28^{\prime}\right)$ & $6534(4)$ & $3499(2)$ & $2477(2)$ & $35(1)$ \\
\hline$C\left(29^{\prime}\right)$ & $3472(4)$ & $4220(2)$ & $1384(2)$ & $25(1)$ \\
\hline$C\left(30^{\prime}\right)$ & $4166(5)$ & $4810(2)$ & $999(2)$ & $43(1)$ \\
\hline$C\left(31^{\prime}\right)$ & $3707(6)$ & $5373(2)$ & $360(2)$ & $57(2)$ \\
\hline$C\left(32^{\prime}\right)$ & $2596(5)$ & $5347(3)$ & $104(2)$ & $55(1)$ \\
\hline$C\left(33^{\prime}\right)$ & $1918(4)$ & $4762(3)$ & $472(2)$ & $49(1)$ \\
\hline$C\left(34^{\prime}\right)$ & $2360(4)$ & $4198(2)$ & $1113(2)$ & $40(1)$ \\
\hline$C\left(35^{\prime}\right)$ & $5130(5)$ & $2820(2)$ & $1025(2)$ & $34(1)$ \\
\hline$C\left(36^{\prime}\right)$ & $5265(4)$ & $2034(2)$ & $1217(2)$ & $28(1)$ \\
\hline$C\left(37^{\prime}\right)$ & $6323(4)$ & $1548(2)$ & $1624(2)$ & $24(1)$ \\
\hline$C\left(38^{\prime}\right)$ & $6416(4)$ & $765(2)$ & $1905(2)$ & $31(1)$ \\
\hline$C\left(39^{\prime}\right)$ & $5470(4)$ & $466(2)$ & $1793(2)$ & $41(1)$ \\
\hline$C\left(40^{\prime}\right)$ & $4400(4)$ & $930(2)$ & $1402(2)$ & $40(1)$ \\
\hline$C\left(41^{\prime}\right)$ & $4273(4)$ & $1697(2)$ & $1128(2)$ & $35(1)$ \\
\hline $\mathrm{S}(1)$ & $-5031(1)$ & $7350(1)$ & $3523(1)$ & $40(1)$ \\
\hline $\mathrm{O}(1)$ & $-4625(3)$ & $7908(2)$ & $2867(2)$ & $78(1)$ \\
\hline $\mathrm{O}(2)$ & $-4574(3)$ & $7350(2)$ & $4129(2)$ & $65(1)$ \\
\hline $\mathrm{O}(3)$ & $-6372(3)$ & $7260(2)$ & $3587(2)$ & $56(1)$ \\
\hline $\mathrm{C}(42)$ & $-4124(5)$ & $6477(3)$ & $3528(3)$ & $49(1)$ \\
\hline $\mathrm{F}(1)$ & $-4346(3)$ & $6359(2)$ & $2987(2)$ & $78(1)$ \\
\hline $\mathrm{F}(2)$ & $-4403(3)$ & $5885(2)$ & $4106(2)$ & $90(1)$ \\
\hline $\mathrm{F}(3)$ & $-2822(3)$ & $6450(2)$ & $3500(2)$ & $78(1)$ \\
\hline$S\left(1^{\prime}\right)$ & $-108(1)$ & $2712(1)$ & $1386(1)$ & $36(1)$ \\
\hline $\mathrm{O}\left(1^{\prime}\right)$ & $-365(3)$ & $3450(2)$ & $1379(2)$ & $49(1)$ \\
\hline $\mathrm{O}\left(2^{\prime}\right)$ & 897(3) & $2174(2)$ & $1831(1)$ & $52(1)$ \\
\hline $\mathrm{O}\left(3^{\prime}\right)$ & $-1263(3)$ & $2423(2)$ & $1407(2)$ & $63(1)$ \\
\hline$C\left(42^{\prime}\right)$ & $624(4)$ & $2878(2)$ & $510(2)$ & $37(1)$ \\
\hline
\end{tabular}




\begin{tabular}{lrrrr}
$\mathrm{F}\left(1^{\prime}\right)$ & $-217(2)$ & $3317(1)$ & $11(1)$ & $53(1)$ \\
$\mathrm{F}\left(2^{\prime}\right)$ & $1032(2)$ & $2236(1)$ & $434(1)$ & $46(1)$ \\
$\mathrm{F}\left(3^{\prime}\right)$ & $1684(2)$ & $3212(1)$ & $367(1)$ & $52(1)$ \\
$\mathrm{C}(43)$ & $-6675(4)$ & $6655(2)$ & $5396(2)$ & $54(1)$ \\
$\mathrm{Cl}(1)$ & $-7566(2)$ & $6006(1)$ & $5399(1)$ & $78(1)$ \\
$\mathrm{Cl}(2)$ & $-7706(1)$ & $7461(1)$ & $5430(1)$ & $65(1)$ \\
\hline
\end{tabular}


Table S18. Bond lengths $[\AA ̊]$ and angles $\left[{ }^{\circ}\right]$ for 9.

\begin{tabular}{|c|c|c|c|}
\hline $\mathrm{Pd}(1)-\mathrm{C}(35)$ & $2.093(4)$ & $\mathrm{C}(11)-\mathrm{C}(12)$ & $1.397(5)$ \\
\hline $\operatorname{Pd}(1)-C(36)$ & $2.251(3)$ & $C(12)-C(13)$ & $1.377(5)$ \\
\hline $\mathrm{Pd}(1)-\mathrm{P}(2)$ & $2.2733(11)$ & $C(13)-C(14)$ & $1.384(5)$ \\
\hline $\mathrm{Pd}(1)-\mathrm{P}(1)$ & $2.3470(14)$ & $C(14)-C(15)$ & $1.378(5)$ \\
\hline $\operatorname{Pd}(1)-C(37)$ & $2.418(4)$ & $C(15)-C(16)$ & $1.387(5)$ \\
\hline $\mathrm{Fe}(1)-\operatorname{cent}(1)$ & $1.711(4)$ & $C(17)-C(18)$ & $1.392(4)$ \\
\hline $\mathrm{Fe}(1)-\operatorname{cent}(2)$ & $1.706(4)$ & $C(17)-C(22)$ & $1.397(5)$ \\
\hline $\mathrm{Fe}(1)-\mathrm{C}(6)$ & $2.023(4)$ & $C(18)-C(19)$ & $1.388(5)$ \\
\hline $\mathrm{Fe}(1)-\mathrm{C}(2)$ & $2.024(4)$ & $C(19)-C(20)$ & $1.376(5)$ \\
\hline $\mathrm{Fe}(1)-\mathrm{C}(1)$ & $2.030(3)$ & $C(20)-C(21)$ & $1.392(5)$ \\
\hline $\mathrm{Fe}(1)-\mathrm{C}(10)$ & $2.040(4)$ & $\mathrm{C}(21)-\mathrm{C}(22)$ & $1.378(5)$ \\
\hline $\mathrm{Fe}(1)-\mathrm{C}(7)$ & $2.039(4)$ & $\mathrm{C}(23)-\mathrm{C}(24)$ & $1.387(5)$ \\
\hline $\mathrm{Fe}(1)-\mathrm{C}(5)$ & $2.048(3)$ & $\mathrm{C}(23)-\mathrm{C}(28)$ & $1.392(5)$ \\
\hline $\mathrm{Fe}(1)-\mathrm{C}(9)$ & $2.051(4)$ & $C(24)-C(25)$ & $1.384(5)$ \\
\hline $\mathrm{Fe}(1)-\mathrm{C}(3)$ & $2.053(4)$ & $C(25)-C(26)$ & $1.393(5)$ \\
\hline $\mathrm{Fe}(1)-\mathrm{C}(8)$ & $2.055(4)$ & $C(26)-C(27)$ & $1.370(5)$ \\
\hline $\mathrm{Fe}(1)-\mathrm{C}(4)$ & $2.067(4)$ & $C(27)-C(28)$ & $1.384(5)$ \\
\hline $\mathrm{P}(1)-\mathrm{C}(1)$ & $1.810(3)$ & $\mathrm{C}(29)-\mathrm{C}(34)$ & $1.393(5)$ \\
\hline$P(1)-C(17)$ & $1.813(3)$ & $C(29)-C(30)$ & $1.392(5)$ \\
\hline $\mathrm{P}(1)-\mathrm{C}(11)$ & $1.814(4)$ & $\mathrm{C}(30)-\mathrm{C}(31)$ & $1.394(5)$ \\
\hline$P(2)-C(6)$ & $1.795(4)$ & $C(31)-C(32)$ & $1.374(5)$ \\
\hline $\mathrm{P}(2)-\mathrm{C}(29)$ & $1.816(4)$ & $C(32)-C(33)$ & $1.376(5)$ \\
\hline$P(2)-C(23)$ & $1.817(4)$ & $\mathrm{C}(33)-\mathrm{C}(34)$ & $1.396(5)$ \\
\hline$C(1)-C(5)$ & $1.418(5)$ & $\mathrm{C}(35)-\mathrm{H}(35 \mathrm{~A})$ & $0.85(3)$ \\
\hline $\mathrm{C}(1)-\mathrm{C}(2)$ & $1.429(5)$ & $\mathrm{C}(35)-\mathrm{H}(35 \mathrm{~B})$ & $1.02(4)$ \\
\hline$C(2)-C(3)$ & $1.412(5)$ & $C(35)-C(36)$ & $1.425(5)$ \\
\hline$C(3)-C(4)$ & $1.419(5)$ & $C(36)-C(41)$ & $1.420(5)$ \\
\hline$C(4)-C(5)$ & $1.416(5)$ & $\mathrm{C}(36)-\mathrm{C}(37)$ & $1.415(5)$ \\
\hline$C(6)-C(10)$ & $1.439(5)$ & $\mathrm{C}(37)-\mathrm{H}(37 \mathrm{~A})$ & $0.96(3)$ \\
\hline$C(6)-C(7)$ & $1.441(5)$ & $\mathrm{C}(37)-\mathrm{C}(38)$ & $1.403(5)$ \\
\hline$C(7)-C(8)$ & $1.418(5)$ & $\mathrm{C}(38)-\mathrm{C}(39)$ & $1.360(5)$ \\
\hline$C(8)-C(9)$ & $1.409(5)$ & $C(39)-C(40)$ & $1.386(5)$ \\
\hline C(9)-C(10) & $1.418(5)$ & $\mathrm{C}(40)-\mathrm{C}(41)$ & $1.364(5)$ \\
\hline$C(11)-C(16)$ & $1.384(5)$ & $\operatorname{Pd}\left(1^{\prime}\right)-C\left(35^{\prime}\right)$ & $2.110(4)$ \\
\hline
\end{tabular}




\begin{tabular}{|c|c|c|c|}
\hline $\operatorname{Pd}\left(1^{\prime}\right)-C\left(36^{\prime}\right)$ & $2.240(4)$ & $\mathrm{C}\left(14^{\prime}\right)-\mathrm{C}\left(15^{\prime}\right)$ & $1.381(5)$ \\
\hline $\operatorname{Pd}\left(1^{\prime}\right)-\mathrm{P}\left(2^{\prime}\right)$ & $2.2857(11)$ & $C\left(15^{\prime}\right)-C\left(16^{\prime}\right)$ & $1.386(5)$ \\
\hline $\operatorname{Pd}\left(1^{\prime}\right)-\mathrm{P}\left(1^{\prime}\right)$ & $2.3443(14)$ & $C\left(17^{\prime}\right)-C\left(22^{\prime}\right)$ & $1.387(5)$ \\
\hline $\operatorname{Pd}\left(1^{\prime}\right)-C\left(37^{\prime}\right)$ & $2.374(4)$ & $C\left(17^{\prime}\right)-C\left(18^{\prime}\right)$ & $1.387(5)$ \\
\hline $\mathrm{Fe}\left(1^{\prime}\right)-\operatorname{cent}\left(1^{\prime}\right)$ & $1.711(4)$ & $\mathrm{C}\left(18^{\prime}\right)-\mathrm{C}\left(19^{\prime}\right)$ & $1.385(5)$ \\
\hline $\operatorname{Fe}\left(1^{\prime}\right)-\operatorname{cent}\left(2^{\prime}\right)$ & $1.706(4)$ & $\mathrm{C}\left(19^{\prime}\right)-\mathrm{C}\left(20^{\prime}\right)$ & $1.376(5)$ \\
\hline $\mathrm{Fe}\left(1^{\prime}\right)-\mathrm{C}\left(6^{\prime}\right)$ & $2.020(3)$ & $C\left(20^{\prime}\right)-C\left(21^{\prime}\right)$ & $1.389(5)$ \\
\hline $\mathrm{Fe}\left(1^{\prime}\right)-\mathrm{C}\left(1^{\prime}\right)$ & $2.028(3)$ & $\mathrm{C}\left(21^{\prime}\right)-\mathrm{C}\left(22^{\prime}\right)$ & $1.393(5)$ \\
\hline $\mathrm{Fe}\left(1^{\prime}\right)-\mathrm{C}\left(2^{\prime}\right)$ & $2.032(3)$ & $\mathrm{C}\left(23^{\prime}\right)-\mathrm{C}\left(28^{\prime}\right)$ & $1.389(5)$ \\
\hline $\operatorname{Fe}\left(1^{\prime}\right)-\mathrm{C}\left(7^{\prime}\right)$ & $2.032(3)$ & $\mathrm{C}\left(23^{\prime}\right)-\mathrm{C}\left(24^{\prime}\right)$ & $1.399(5)$ \\
\hline $\mathrm{Fe}\left(1^{\prime}\right)-\mathrm{C}\left(10^{\prime}\right)$ & $2.041(3)$ & $\mathrm{C}\left(24^{\prime}\right)-\mathrm{C}\left(25^{\prime}\right)$ & $1.384(5)$ \\
\hline $\mathrm{Fe}\left(1^{\prime}\right)-\mathrm{C}\left(5^{\prime}\right)$ & $2.045(3)$ & $\mathrm{C}\left(25^{\prime}\right)-\mathrm{C}\left(26^{\prime}\right)$ & $1.380(6)$ \\
\hline $\mathrm{Fe}\left(1^{\prime}\right)-\mathrm{C}\left(4^{\prime}\right)$ & $2.061(4)$ & $\mathrm{C}\left(26^{\prime}\right)-\mathrm{C}\left(27^{\prime}\right)$ & $1.380(5)$ \\
\hline $\mathrm{Fe}\left(1^{\prime}\right)-\mathrm{C}\left(8^{\prime}\right)$ & $2.057(3)$ & $\mathrm{C}\left(27^{\prime}\right)-\mathrm{C}\left(28^{\prime}\right)$ & $1.383(5)$ \\
\hline $\mathrm{Fe}\left(1^{\prime}\right)-\mathrm{C}\left(3^{\prime}\right)$ & $2.059(4)$ & $\mathrm{C}\left(29^{\prime}\right)-\mathrm{C}\left(34^{\prime}\right)$ & $1.384(5)$ \\
\hline $\mathrm{Fe}\left(1^{\prime}\right)-\mathrm{C}\left(9^{\prime}\right)$ & $2.063(4)$ & $\mathrm{C}\left(29^{\prime}\right)-\mathrm{C}\left(30^{\prime}\right)$ & $1.393(5)$ \\
\hline $\mathrm{P}\left(1^{\prime}\right)-\mathrm{C}\left(1^{\prime}\right)$ & $1.804(3)$ & $\mathrm{C}\left(30^{\prime}\right)-\mathrm{C}\left(31^{\prime}\right)$ & $1.395(6)$ \\
\hline $\mathrm{P}\left(1^{\prime}\right)-\mathrm{C}\left(17^{\prime}\right)$ & $1.821(3)$ & $\mathrm{C}\left(31^{\prime}\right)-\mathrm{C}\left(32^{\prime}\right)$ & $1.368(6)$ \\
\hline $\mathrm{P}\left(1^{\prime}\right)-\mathrm{C}\left(11^{\prime}\right)$ & $1.826(4)$ & $\mathrm{C}\left(32^{\prime}\right)-\mathrm{C}\left(33^{\prime}\right)$ & $1.370(6)$ \\
\hline $\mathrm{P}\left(2^{\prime}\right)-\mathrm{C}\left(6^{\prime}\right)$ & $1.798(4)$ & $\mathrm{C}\left(33^{\prime}\right)-\mathrm{C}\left(34^{\prime}\right)$ & $1.395(5)$ \\
\hline $\mathrm{P}\left(2^{\prime}\right)-\mathrm{C}\left(23^{\prime}\right)$ & $1.804(4)$ & $\mathrm{C}\left(35^{\prime}\right)-\mathrm{H}(35 \mathrm{D})$ & $0.95(4)$ \\
\hline$P\left(2^{\prime}\right)-C\left(29^{\prime}\right)$ & $1.828(4)$ & $\mathrm{C}\left(35^{\prime}\right)-\mathrm{H}(35 \mathrm{C})$ & $0.92(3)$ \\
\hline $\mathrm{C}\left(1^{\prime}\right)-\mathrm{C}\left(2^{\prime}\right)$ & $1.426(5)$ & $\mathrm{C}\left(35^{\prime}\right)-\mathrm{C}\left(36^{\prime}\right)$ & $1.424(5)$ \\
\hline$C\left(1^{\prime}\right)-C\left(5^{\prime}\right)$ & $1.441(5)$ & $C\left(36^{\prime}\right)-C\left(37^{\prime}\right)$ & $1.406(5)$ \\
\hline $\mathrm{C}\left(2^{\prime}\right)-\mathrm{C}\left(3^{\prime}\right)$ & $1.427(5)$ & $C\left(36^{\prime}\right)-C\left(41^{\prime}\right)$ & $1.425(5)$ \\
\hline$C\left(3^{\prime}\right)-C\left(4^{\prime}\right)$ & $1.400(5)$ & $\mathrm{C}\left(37^{\prime}\right)-\mathrm{H}(37 \mathrm{~B})$ & $0.88(3)$ \\
\hline$C\left(4^{\prime}\right)-C\left(5^{\prime}\right)$ & $1.417(5)$ & $\mathrm{C}\left(37^{\prime}\right)-\mathrm{C}\left(38^{\prime}\right)$ & $1.399(5)$ \\
\hline$C\left(6^{\prime}\right)-C\left(10^{\prime}\right)$ & $1.427(5)$ & $\mathrm{C}\left(38^{\prime}\right)-\mathrm{C}\left(39^{\prime}\right)$ & $1.357(5)$ \\
\hline$C\left(6^{\prime}\right)-C\left(7^{\prime}\right)$ & $1.443(5)$ & $\mathrm{C}\left(39^{\prime}\right)-\mathrm{C}\left(40^{\prime}\right)$ & $1.391(5)$ \\
\hline$C\left(7^{\prime}\right)-C\left(8^{\prime}\right)$ & $1.416(5)$ & $C\left(40^{\prime}\right)-C\left(41^{\prime}\right)$ & $1.368(5)$ \\
\hline $\mathrm{C}\left(8^{\prime}\right)-\mathrm{C}\left(9^{\prime}\right)$ & $1.406(5)$ & $\mathrm{S}(1)-\mathrm{O}(1)$ & $1.426(3)$ \\
\hline $\mathrm{C}\left(9^{\prime}\right)-\mathrm{C}\left(10^{\prime}\right)$ & $1.419(5)$ & $\mathrm{S}(1)-\mathrm{O}(3)$ & $1.428(3)$ \\
\hline$C\left(11^{\prime}\right)-C\left(16^{\prime}\right)$ & $1.389(5)$ & $\mathrm{S}(1)-\mathrm{O}(2)$ & $1.434(3)$ \\
\hline$C\left(11^{\prime}\right)-C\left(12^{\prime}\right)$ & $1.397(5)$ & $S(1)-C(42)$ & $1.797(5)$ \\
\hline $\mathrm{C}\left(12^{\prime}\right)-\mathrm{C}\left(13^{\prime}\right)$ & $1.384(5)$ & $\mathrm{C}(42)-\mathrm{F}(1)$ & $1.328(5)$ \\
\hline$C\left(13^{\prime}\right)-C\left(14^{\prime}\right)$ & $1.376(6)$ & $\mathrm{C}(42)-\mathrm{F}(2)$ & $1.329(5)$ \\
\hline
\end{tabular}




\begin{tabular}{|c|c|c|c|}
\hline$C(42)-F(3)$ & $1.340(5)$ & $C(7)-F e(1)-C(5)$ & $177.65(16)$ \\
\hline $\mathrm{S}\left(1^{\prime}\right)-\mathrm{O}\left(3^{\prime}\right)$ & $1.434(3)$ & $\mathrm{C}(6)-\mathrm{Fe}(1)-\mathrm{C}(9)$ & $68.93(15)$ \\
\hline $\mathrm{S}\left(1^{\prime}\right)-\mathrm{O}\left(2^{\prime}\right)$ & $1.432(3)$ & $\mathrm{C}(2)-\mathrm{Fe}(1)-\mathrm{C}(9)$ & $177.21(15)$ \\
\hline $\mathrm{S}\left(1^{\prime}\right)-\mathrm{O}\left(1^{\prime}\right)$ & $1.439(3)$ & $\mathrm{C}(1)-\mathrm{Fe}(1)-\mathrm{C}(9)$ & $141.48(15)$ \\
\hline $\mathrm{S}\left(1^{\prime}\right)-\mathrm{C}\left(42^{\prime}\right)$ & $1.819(4)$ & $\mathrm{C}(10)-\mathrm{Fe}(1)-\mathrm{C}(9)$ & $40.55(14)$ \\
\hline$C\left(42^{\prime}\right)-F\left(1^{\prime}\right)$ & $1.333(4)$ & $\mathrm{C}(7)-\mathrm{Fe}(1)-\mathrm{C}(9)$ & $68.34(16)$ \\
\hline $\mathrm{C}\left(42^{\prime}\right)-\mathrm{F}\left(2^{\prime}\right)$ & $1.337(4)$ & $\mathrm{C}(5)-\mathrm{Fe}(1)-\mathrm{C}(9)$ & $113.73(16)$ \\
\hline $\mathrm{C}\left(42^{\prime}\right)-\mathrm{F}\left(3^{\prime}\right)$ & $1.346(4)$ & $\mathrm{C}(6)-\mathrm{Fe}(1)-\mathrm{C}(3)$ & $138.88(15)$ \\
\hline $\mathrm{C}(43)-\mathrm{Cl}(1)$ & $1.753(4)$ & $\mathrm{C}(2)-\mathrm{Fe}(1)-\mathrm{C}(3)$ & $40.50(13)$ \\
\hline \multirow[t]{2}{*}{$\mathrm{C}(43)-\mathrm{Cl}(2)$} & $1.759(4)$ & $\mathrm{C}(1)-\mathrm{Fe}(1)-\mathrm{C}(3)$ & $68.84(14)$ \\
\hline & & $\mathrm{C}(10)-\mathrm{Fe}(1)-\mathrm{C}(3)$ & $178.29(15)$ \\
\hline$C(35)-P d(1)-C(36)$ & $38.07(14)$ & $\mathrm{C}(7)-\mathrm{Fe}(1)-\mathrm{C}(3)$ & $109.94(16)$ \\
\hline $\mathrm{C}(35)-\mathrm{Pd}(1)-\mathrm{P}(2)$ & $92.41(12)$ & $\mathrm{C}(5)-\mathrm{Fe}(1)-\mathrm{C}(3)$ & $67.82(15)$ \\
\hline $\mathrm{C}(36)-\mathrm{Pd}(1)-\mathrm{P}(2)$ & $129.24(10)$ & $\mathrm{C}(9)-\mathrm{Fe}(1)-\mathrm{C}(3)$ & $137.81(16)$ \\
\hline $\mathrm{C}(35)-\mathrm{Pd}(1)-\mathrm{P}(1)$ & $164.40(12)$ & $\mathrm{C}(6)-\mathrm{Fe}(1)-\mathrm{C}(8)$ & $68.89(15)$ \\
\hline $\mathrm{C}(36)-\mathrm{Pd}(1)-\mathrm{P}(1)$ & $126.50(9)$ & $\mathrm{C}(2)-\mathrm{Fe}(1)-\mathrm{C}(8)$ & $137.11(15)$ \\
\hline $\mathrm{P}(2)-\mathrm{Pd}(1)-\mathrm{P}(1)$ & $101.95(4)$ & $\mathrm{C}(1)-\mathrm{Fe}(1)-\mathrm{C}(8)$ & $178.33(16)$ \\
\hline$C(35)-P d(1)-C(37)$ & $65.01(15)$ & $\mathrm{C}(10)-\mathrm{Fe}(1)-\mathrm{C}(8)$ & $68.25(15)$ \\
\hline$C(36)-P d(1)-C(37)$ & $35.04(12)$ & $\mathrm{C}(7)-\mathrm{Fe}(1)-\mathrm{C}(8)$ & $40.52(14)$ \\
\hline $\mathrm{P}(2)-\mathrm{Pd}(1)-\mathrm{C}(37)$ & $152.53(10)$ & $\mathrm{C}(5)-\mathrm{Fe}(1)-\mathrm{C}(8)$ & $140.43(16)$ \\
\hline $\mathrm{P}(1)-\mathrm{Pd}(1)-\mathrm{C}(37)$ & $102.58(10)$ & $\mathrm{C}(9)-\mathrm{Fe}(1)-\mathrm{C}(8)$ & $40.13(14)$ \\
\hline Cent-Fe(1)-cent & $149.2(1)$ & $\mathrm{C}(3)-\mathrm{Fe}(1)-\mathrm{C}(8)$ & $110.15(16)$ \\
\hline$C(6)-F e(1)-C(2)$ & $111.02(15)$ & $C(6)-F e(1)-C(4)$ & $179.06(16)$ \\
\hline$C(6)-F e(1)-C(1)$ & $110.91(14)$ & $\mathrm{C}(2)-\mathrm{Fe}(1)-\mathrm{C}(4)$ & $68.06(15)$ \\
\hline $\mathrm{C}(2)-\mathrm{Fe}(1)-\mathrm{C}(1)$ & $41.28(13)$ & $\mathrm{C}(1)-\mathrm{Fe}(1)-\mathrm{C}(4)$ & $68.56(14)$ \\
\hline$C(6)-F e(1)-C(10)$ & 41.47(14) & $C(10)-F e(1)-C(4)$ & $139.40(16)$ \\
\hline$C(2)-F e(1)-C(10)$ & $141.10(15)$ & $\mathrm{C}(7)-\mathrm{Fe}(1)-\mathrm{C}(4)$ & $138.35(15)$ \\
\hline$C(1)-F e(1)-C(10)$ & $112.78(14)$ & $\mathrm{C}(5)-\mathrm{Fe}(1)-\mathrm{C}(4)$ & $40.26(13)$ \\
\hline$C(6)-F e(1)-C(7)$ & $41.56(13)$ & $\mathrm{C}(9)-\mathrm{Fe}(1)-\mathrm{C}(4)$ & $111.98(16)$ \\
\hline $\mathrm{C}(2)-\mathrm{Fe}(1)-\mathrm{C}(7)$ & 109.67(16) & $\mathrm{C}(3)-\mathrm{Fe}(1)-\mathrm{C}(4)$ & $40.28(14)$ \\
\hline $\mathrm{C}(1)-\mathrm{Fe}(1)-\mathrm{C}(7)$ & $138.28(16)$ & $\mathrm{C}(8)-\mathrm{Fe}(1)-\mathrm{C}(4)$ & $111.62(16)$ \\
\hline$C(10)-F e(1)-C(7)$ & $69.31(15)$ & $C(1)-P(1)-C(17)$ & $102.12(16)$ \\
\hline $\mathrm{C}(6)-\mathrm{Fe}(1)-\mathrm{C}(5)$ & $139.78(14)$ & $\mathrm{C}(1)-\mathrm{P}(1)-\mathrm{C}(11)$ & $101.78(16)$ \\
\hline $\mathrm{C}(2)-\mathrm{Fe}(1)-\mathrm{C}(5)$ & $68.22(15)$ & $C(17)-P(1)-C(11)$ & $106.97(16)$ \\
\hline $\mathrm{C}(1)-\mathrm{Fe}(1)-\mathrm{C}(5)$ & $40.69(14)$ & $\mathrm{C}(1)-\mathrm{P}(1)-\mathrm{Pd}(1)$ & $119.33(12)$ \\
\hline$C(10)-F e(1)-C(5)$ & $112.96(15)$ & $\mathrm{C}(17)-\mathrm{P}(1)-\mathrm{Pd}(1)$ & $106.98(12)$ \\
\hline
\end{tabular}




\begin{tabular}{|c|c|c|c|}
\hline$C(11)-P(1)-P d(1)$ & $118.05(12)$ & $\mathrm{C}(7)-\mathrm{C}(8)-\mathrm{Fe}(1)$ & $69.1(2)$ \\
\hline$C(6)-P(2)-C(29)$ & $104.44(17)$ & $C(8)-C(9)-C(10)$ & $108.7(4)$ \\
\hline$C(6)-P(2)-C(23)$ & $106.17(16)$ & $\mathrm{C}(8)-\mathrm{C}(9)-\mathrm{Fe}(1)$ & $70.1(2)$ \\
\hline $\mathrm{C}(29)-\mathrm{P}(2)-\mathrm{C}(23)$ & $104.09(17)$ & $\mathrm{C}(10)-\mathrm{C}(9)-\mathrm{Fe}(1)$ & $69.3(2)$ \\
\hline$C(6)-P(2)-P d(1)$ & $112.51(12)$ & $C(9)-C(10)-C(6)$ & $107.7(3)$ \\
\hline $\mathrm{C}(29)-\mathrm{P}(2)-\mathrm{Pd}(1)$ & $114.82(12)$ & $\mathrm{C}(9)-\mathrm{C}(10)-\mathrm{Fe}(1)$ & $70.1(2)$ \\
\hline$C(23)-P(2)-P d(1)$ & $113.86(12)$ & $C(6)-C(10)-F e(1)$ & $68.6(2)$ \\
\hline$C(5)-C(1)-C(2)$ & $106.7(3)$ & $C(16)-C(11)-C(12)$ & $118.6(3)$ \\
\hline$C(5)-C(1)-P(1)$ & $128.5(3)$ & $\mathrm{C}(16)-\mathrm{C}(11)-\mathrm{P}(1)$ & $119.4(3)$ \\
\hline $\mathrm{C}(2)-\mathrm{C}(1)-\mathrm{P}(1)$ & $124.8(3)$ & $\mathrm{C}(12)-\mathrm{C}(11)-\mathrm{P}(1)$ & $121.8(3)$ \\
\hline $\mathrm{C}(5)-\mathrm{C}(1)-\mathrm{Fe}(1)$ & $70.3(2)$ & $C(13)-C(12)-C(11)$ & $120.2(4)$ \\
\hline $\mathrm{C}(2)-\mathrm{C}(1)-\mathrm{Fe}(1)$ & 69.13(19) & $C(12)-C(13)-C(14)$ & $120.4(4)$ \\
\hline $\mathrm{P}(1)-\mathrm{C}(1)-\mathrm{Fe}(1)$ & $125.94(18)$ & $C(15)-C(14)-C(13)$ & $120.1(4)$ \\
\hline $\mathrm{C}(3)-\mathrm{C}(2)-\mathrm{C}(1)$ & $108.7(3)$ & $C(14)-C(15)-C(16)$ & $119.4(4)$ \\
\hline $\mathrm{C}(3)-\mathrm{C}(2)-\mathrm{Fe}(1)$ & $70.9(2)$ & $C(11)-C(16)-C(15)$ & $121.2(4)$ \\
\hline $\mathrm{C}(1)-\mathrm{C}(2)-\mathrm{Fe}(1)$ & $69.6(2)$ & $\mathrm{C}(18)-\mathrm{C}(17)-\mathrm{C}(22)$ & $118.5(3)$ \\
\hline$C(2)-C(3)-C(4)$ & $108.0(3)$ & $\mathrm{C}(18)-\mathrm{C}(17)-\mathrm{P}(1)$ & $124.3(3)$ \\
\hline $\mathrm{C}(2)-\mathrm{C}(3)-\mathrm{Fe}(1)$ & $68.6(2)$ & $\mathrm{C}(22)-\mathrm{C}(17)-\mathrm{P}(1)$ & $117.2(3)$ \\
\hline $\mathrm{C}(4)-\mathrm{C}(3)-\mathrm{Fe}(1)$ & $70.4(2)$ & $C(19)-C(18)-C(17)$ & $120.5(3)$ \\
\hline$C(5)-C(4)-C(3)$ & $107.6(3)$ & $C(20)-C(19)-C(18)$ & $119.9(4)$ \\
\hline$C(5)-C(4)-F e(1)$ & $69.1(2)$ & $C(19)-C(20)-C(21)$ & $120.6(4)$ \\
\hline$C(3)-C(4)-F e(1)$ & $69.3(2)$ & $C(22)-C(21)-C(20)$ & $119.1(4)$ \\
\hline$C(4)-C(5)-C(1)$ & $109.0(3)$ & $C(21)-C(22)-C(17)$ & $121.3(3)$ \\
\hline$C(4)-C(5)-F e(1)$ & $70.6(2)$ & $\mathrm{C}(24)-\mathrm{C}(23)-\mathrm{C}(28)$ & $118.6(3)$ \\
\hline $\mathrm{C}(1)-\mathrm{C}(5)-\mathrm{Fe}(1)$ & $69.0(2)$ & $\mathrm{C}(24)-\mathrm{C}(23)-\mathrm{P}(2)$ & $121.2(3)$ \\
\hline$C(10)-C(6)-C(7)$ & $107.3(3)$ & $\mathrm{C}(28)-\mathrm{C}(23)-\mathrm{P}(2)$ & $120.1(3)$ \\
\hline$C(10)-C(6)-P(2)$ & $123.3(3)$ & $C(25)-C(24)-C(23)$ & $120.7(4)$ \\
\hline$C(7)-C(6)-P(2)$ & $129.4(3)$ & $C(24)-C(25)-C(26)$ & $120.0(4)$ \\
\hline $\mathrm{C}(10)-\mathrm{C}(6)-\mathrm{Fe}(1)$ & $69.9(2)$ & $C(27)-C(26)-C(25)$ & $119.6(4)$ \\
\hline$C(7)-C(6)-F e(1)$ & $69.8(2)$ & $C(26)-C(27)-C(28)$ & $120.6(4)$ \\
\hline $\mathrm{P}(2)-\mathrm{C}(6)-\mathrm{Fe}(1)$ & $124.72(19)$ & $\mathrm{C}(23)-\mathrm{C}(28)-\mathrm{C}(27)$ & $120.6(3)$ \\
\hline$C(8)-C(7)-C(6)$ & $107.6(3)$ & $C(34)-C(29)-C(30)$ & $119.0(3)$ \\
\hline $\mathrm{C}(8)-\mathrm{C}(7)-\mathrm{Fe}(1)$ & $70.3(2)$ & $\mathrm{C}(34)-\mathrm{C}(29)-\mathrm{P}(2)$ & $120.2(3)$ \\
\hline $\mathrm{C}(6)-\mathrm{C}(7)-\mathrm{Fe}(1)$ & $68.63(19)$ & $\mathrm{C}(30)-\mathrm{C}(29)-\mathrm{P}(2)$ & $120.5(3)$ \\
\hline $\mathrm{C}(9)-\mathrm{C}(8)-\mathrm{C}(7)$ & $108.7(3)$ & $\mathrm{C}(29)-\mathrm{C}(30)-\mathrm{C}(31)$ & $120.1(4)$ \\
\hline $\mathrm{C}(9)-\mathrm{C}(8)-\mathrm{Fe}(1)$ & $69.8(2)$ & $C(32)-C(31)-C(30)$ & $120.6(4)$ \\
\hline
\end{tabular}




\begin{tabular}{|c|c|c|c|}
\hline$C(31)-C(32)-C(33)$ & $119.8(4)$ & $C\left(6^{\prime}\right)-\mathrm{Fe}\left(1^{\prime}\right)-\mathrm{C}\left(1^{\prime}\right)$ & $111.63(14)$ \\
\hline $\mathrm{C}(32)-\mathrm{C}(33)-\mathrm{C}(34)$ & $120.5(4)$ & $\mathrm{C}\left(6^{\prime}\right)-\mathrm{Fe}\left(1^{\prime}\right)-\mathrm{C}\left(2^{\prime}\right)$ & $109.22(15)$ \\
\hline $\mathrm{C}(29)-\mathrm{C}(34)-\mathrm{C}(33)$ & $120.0(4)$ & $\mathrm{C}\left(1^{\prime}\right)-\mathrm{Fe}\left(1^{\prime}\right)-\mathrm{C}\left(2^{\prime}\right)$ & $41.11(13)$ \\
\hline $\mathrm{H}(35 \mathrm{~A})-\mathrm{C}(35)-\mathrm{H}(35 \mathrm{~B})$ & $116(3)$ & $\mathrm{C}\left(6^{\prime}\right)-\mathrm{Fe}\left(1^{\prime}\right)-\mathrm{C}\left(7^{\prime}\right)$ & $41.72(13)$ \\
\hline $\mathrm{H}(35 \mathrm{~A})-\mathrm{C}(35)-\mathrm{C}(36)$ & $121(2)$ & $\mathrm{C}\left(1^{\prime}\right)-\mathrm{Fe}\left(1^{\prime}\right)-\mathrm{C}\left(7^{\prime}\right)$ & $140.55(15)$ \\
\hline $\mathrm{H}(35 \mathrm{~B})-\mathrm{C}(35)-\mathrm{C}(36)$ & $114(2)$ & $\mathrm{C}\left(2^{\prime}\right)-\mathrm{Fe}\left(1^{\prime}\right)-\mathrm{C}\left(7^{\prime}\right)$ & $110.47(15)$ \\
\hline $\mathrm{H}(35 \mathrm{~A})-\mathrm{C}(35)-\mathrm{Pd}(1)$ & $105(2)$ & $\mathrm{C}\left(6^{\prime}\right)-\mathrm{Fe}\left(1^{\prime}\right)-\mathrm{C}\left(10^{\prime}\right)$ & $41.13(13)$ \\
\hline $\mathrm{H}(35 \mathrm{~B})-\mathrm{C}(35)-\mathrm{Pd}(1)$ & $118(2)$ & $\mathrm{C}\left(1^{\prime}\right)-\mathrm{Fe}\left(1^{\prime}\right)-\mathrm{C}\left(10^{\prime}\right)$ & $111.71(14)$ \\
\hline $\mathrm{C}(36)-\mathrm{C}(35)-\mathrm{Pd}(1)$ & $77.0(2)$ & $\mathrm{C}\left(2^{\prime}\right)-\mathrm{Fe}\left(1^{\prime}\right)-\mathrm{C}\left(10^{\prime}\right)$ & $137.86(14)$ \\
\hline $\mathrm{C}(41)-\mathrm{C}(36)-\mathrm{C}(37)$ & 117.2(3) & $\mathrm{C}\left(7^{\prime}\right)-\mathrm{Fe}\left(1^{\prime}\right)-\mathrm{C}\left(10^{\prime}\right)$ & $69.04(14)$ \\
\hline $\mathrm{C}(41)-\mathrm{C}(36)-\mathrm{C}(35)$ & $123.1(4)$ & $\mathrm{C}\left(6^{\prime}\right)-\mathrm{Fe}\left(1^{\prime}\right)-\mathrm{C}\left(5^{\prime}\right)$ & $142.62(14)$ \\
\hline$C(37)-C(36)-C(35)$ & $118.4(4)$ & $\mathrm{C}\left(1^{\prime}\right)-\mathrm{Fe}\left(1^{\prime}\right)-\mathrm{C}\left(5^{\prime}\right)$ & $41.45(13)$ \\
\hline$C(41)-C(36)-\operatorname{Pd}(1)$ & $116.6(2)$ & $\mathrm{C}\left(2^{\prime}\right)-\mathrm{Fe}\left(1^{\prime}\right)-\mathrm{C}\left(5^{\prime}\right)$ & $68.78(15)$ \\
\hline$C(37)-C(36)-P d(1)$ & $78.9(2)$ & $\mathrm{C}\left(7^{\prime}\right)-\mathrm{Fe}\left(1^{\prime}\right)-\mathrm{C}\left(5^{\prime}\right)$ & $175.65(14)$ \\
\hline$C(35)-C(36)-P d(1)$ & $64.9(2)$ & $C\left(10^{\prime}\right)-\mathrm{Fe}\left(1^{\prime}\right)-\mathrm{C}\left(5^{\prime}\right)$ & $114.54(14)$ \\
\hline $\mathrm{H}(37 \mathrm{~A})-\mathrm{C}(37)-\mathrm{C}(38)$ & $117(2)$ & $\mathrm{C}\left(6^{\prime}\right)-\mathrm{Fe}\left(1^{\prime}\right)-\mathrm{C}\left(4^{\prime}\right)$ & $175.61(15)$ \\
\hline $\mathrm{H}(37 \mathrm{~A})-\mathrm{C}(37)-\mathrm{C}(36)$ & $122(2)$ & $\mathrm{C}\left(1^{\prime}\right)-\mathrm{Fe}\left(1^{\prime}\right)-\mathrm{C}\left(4^{\prime}\right)$ & $68.80(14)$ \\
\hline C(38)-C(37)-C(36) & $120.8(4)$ & $\mathrm{C}\left(2^{\prime}\right)-\mathrm{Fe}\left(1^{\prime}\right)-\mathrm{C}\left(4^{\prime}\right)$ & $68.04(16)$ \\
\hline $\mathrm{H}(37 \mathrm{~A})-\mathrm{C}(37)-\mathrm{Pd}(1)$ & $87(2)$ & $\mathrm{C}\left(7^{\prime}\right)-\mathrm{Fe}\left(1^{\prime}\right)-\mathrm{C}\left(4^{\prime}\right)$ & $135.28(15)$ \\
\hline $\mathrm{C}(38)-\mathrm{C}(37)-\mathrm{Pd}(1)$ & $125.5(3)$ & $\mathrm{C}\left(10^{\prime}\right)-\mathrm{Fe}\left(1^{\prime}\right)-\mathrm{C}\left(4^{\prime}\right)$ & $143.13(15)$ \\
\hline$C(36)-C(37)-P d(1)$ & $66.03(19)$ & $\mathrm{C}\left(5^{\prime}\right)-\mathrm{Fe}\left(1^{\prime}\right)-\mathrm{C}\left(4^{\prime}\right)$ & $40.37(13)$ \\
\hline $\mathrm{C}(39)-\mathrm{C}(38)-\mathrm{C}(37)$ & $119.6(4)$ & $\mathrm{C}\left(6^{\prime}\right)-\mathrm{Fe}\left(1^{\prime}\right)-\mathrm{C}\left(8^{\prime}\right)$ & $68.71(14)$ \\
\hline$C(38)-C(39)-C(40)$ & $120.6(4)$ & $\mathrm{C}\left(1^{\prime}\right)-\mathrm{Fe}\left(1^{\prime}\right)-\mathrm{C}\left(8^{\prime}\right)$ & $178.66(15)$ \\
\hline$C(41)-C(40)-C(39)$ & $121.3(4)$ & $\mathrm{C}\left(2^{\prime}\right)-\mathrm{Fe}\left(1^{\prime}\right)-\mathrm{C}\left(8^{\prime}\right)$ & $140.15(14)$ \\
\hline$C(40)-C(41)-C(36)$ & $120.3(4)$ & $\mathrm{C}\left(7^{\prime}\right)-\mathrm{Fe}\left(1^{\prime}\right)-\mathrm{C}\left(8^{\prime}\right)$ & $40.52(14)$ \\
\hline$C\left(35^{\prime}\right)-P d\left(1^{\prime}\right)-C\left(36^{\prime}\right)$ & $38.06(14)$ & $C\left(10^{\prime}\right)-\mathrm{Fe}\left(1^{\prime}\right)-\mathrm{C}\left(8^{\prime}\right)$ & $67.62(14)$ \\
\hline $\mathrm{C}\left(35^{\prime}\right)-\mathrm{Pd}\left(1^{\prime}\right)-\mathrm{P}\left(2^{\prime}\right)$ & $93.98(12)$ & $\mathrm{C}\left(5^{\prime}\right)-\mathrm{Fe}\left(1^{\prime}\right)-\mathrm{C}\left(8^{\prime}\right)$ & $137.57(15)$ \\
\hline$C\left(36^{\prime}\right)-P d\left(1^{\prime}\right)-P\left(2^{\prime}\right)$ & 129.64(10) & $\mathrm{C}\left(4^{\prime}\right)-\mathrm{Fe}\left(1^{\prime}\right)-\mathrm{C}\left(8^{\prime}\right)$ & $110.97(15)$ \\
\hline$C\left(35^{\prime}\right)-P d\left(1^{\prime}\right)-P\left(1^{\prime}\right)$ & $163.82(12)$ & $C\left(6^{\prime}\right)-\mathrm{Fe}\left(1^{\prime}\right)-\mathrm{C}\left(3^{\prime}\right)$ & $135.99(16)$ \\
\hline $\mathrm{C}\left(36^{\prime}\right)-\mathrm{Pd}\left(1^{\prime}\right)-\mathrm{P}\left(1^{\prime}\right)$ & $126.23(10)$ & $\mathrm{C}\left(1^{\prime}\right)-\mathrm{Fe}\left(1^{\prime}\right)-\mathrm{C}\left(3^{\prime}\right)$ & $68.87(14)$ \\
\hline $\mathrm{P}\left(2^{\prime}\right)-\mathrm{Pd}\left(1^{\prime}\right)-\mathrm{P}\left(1^{\prime}\right)$ & $102.19(5)$ & $\mathrm{C}\left(2^{\prime}\right)-\mathrm{Fe}\left(1^{\prime}\right)-\mathrm{C}\left(3^{\prime}\right)$ & $40.82(14)$ \\
\hline $\mathrm{C}\left(35^{\prime}\right)-\mathrm{Pd}\left(1^{\prime}\right)-\mathrm{C}\left(37^{\prime}\right)$ & $65.25(15)$ & $\mathrm{C}\left(7^{\prime}\right)-\mathrm{Fe}\left(1^{\prime}\right)-\mathrm{C}\left(3^{\prime}\right)$ & $108.62(15)$ \\
\hline $\mathrm{C}\left(36^{\prime}\right)-\mathrm{Pd}\left(1^{\prime}\right)-\mathrm{C}\left(37^{\prime}\right)$ & $35.35(12)$ & $\mathrm{C}\left(10^{\prime}\right)-\mathrm{Fe}\left(1^{\prime}\right)-\mathrm{C}\left(3^{\prime}\right)$ & $177.11(16)$ \\
\hline $\mathrm{P}\left(2^{\prime}\right)-\mathrm{Pd}\left(1^{\prime}\right)-\mathrm{C}\left(37^{\prime}\right)$ & $156.55(10)$ & $\mathrm{C}\left(5^{\prime}\right)-\mathrm{Fe}\left(1^{\prime}\right)-\mathrm{C}\left(3^{\prime}\right)$ & $67.87(15)$ \\
\hline $\mathrm{P}\left(1^{\prime}\right)-\mathrm{Pd}\left(1^{\prime}\right)-\mathrm{C}\left(37^{\prime}\right)$ & $99.00(10)$ & $\mathrm{C}\left(4^{\prime}\right)-\mathrm{Fe}\left(1^{\prime}\right)-\mathrm{C}\left(3^{\prime}\right)$ & $39.75(15)$ \\
\hline Cent-Fe(1')-cent & $149.8(1)$ & $\mathrm{C}\left(8^{\prime}\right)-\mathrm{Fe}\left(1^{\prime}\right)-\mathrm{C}\left(3^{\prime}\right)$ & $111.85(15)$ \\
\hline
\end{tabular}




\begin{tabular}{|c|c|c|c|}
\hline $\mathrm{C}\left(6^{\prime}\right)-\mathrm{Fe}\left(1^{\prime}\right)-\mathrm{C}\left(9^{\prime}\right)$ & $68.91(15)$ & $\mathrm{C}\left(4^{\prime}\right)-\mathrm{C}\left(5^{\prime}\right)-\mathrm{C}\left(1^{\prime}\right)$ & $107.8(3)$ \\
\hline $\mathrm{C}\left(1^{\prime}\right)-\mathrm{Fe}\left(1^{\prime}\right)-\mathrm{C}\left(9^{\prime}\right)$ & $138.87(14)$ & $\mathrm{C}\left(4^{\prime}\right)-\mathrm{C}\left(5^{\prime}\right)-\mathrm{Fe}\left(1^{\prime}\right)$ & $70.4(2)$ \\
\hline $\mathrm{C}\left(2^{\prime}\right)-\mathrm{Fe}\left(1^{\prime}\right)-\mathrm{C}\left(9^{\prime}\right)$ & $178.10(16)$ & $\mathrm{C}\left(1^{\prime}\right)-\mathrm{C}\left(5^{\prime}\right)-\mathrm{Fe}\left(1^{\prime}\right)$ & $68.63(19)$ \\
\hline $\mathrm{C}\left(7^{\prime}\right)-\mathrm{Fe}\left(1^{\prime}\right)-\mathrm{C}\left(9^{\prime}\right)$ & $68.45(15)$ & $\mathrm{C}\left(10^{\prime}\right)-\mathrm{C}\left(6^{\prime}\right)-\mathrm{C}\left(7^{\prime}\right)$ & 107.1(3) \\
\hline$C\left(10^{\prime}\right)-\mathrm{Fe}\left(1^{\prime}\right)-\mathrm{C}\left(9^{\prime}\right)$ & $40.43(13)$ & $\mathrm{C}\left(10^{\prime}\right)-\mathrm{C}\left(6^{\prime}\right)-\mathrm{P}\left(2^{\prime}\right)$ & $121.4(3)$ \\
\hline $\mathrm{C}\left(5^{\prime}\right)-\mathrm{Fe}\left(1^{\prime}\right)-\mathrm{C}\left(9^{\prime}\right)$ & $112.42(15)$ & $\mathrm{C}\left(7^{\prime}\right)-\mathrm{C}\left(6^{\prime}\right)-\mathrm{P}\left(2^{\prime}\right)$ & $131.5(3)$ \\
\hline $\mathrm{C}\left(4^{\prime}\right)-\mathrm{Fe}\left(1^{\prime}\right)-\mathrm{C}\left(9^{\prime}\right)$ & $113.85(16)$ & $\mathrm{C}\left(10^{\prime}\right)-\mathrm{C}\left(6^{\prime}\right)-\mathrm{Fe}\left(1^{\prime}\right)$ & $70.2(2)$ \\
\hline $\mathrm{C}\left(8^{\prime}\right)-\mathrm{Fe}\left(1^{\prime}\right)-\mathrm{C}\left(9^{\prime}\right)$ & $39.89(13)$ & $\mathrm{C}\left(7^{\prime}\right)-\mathrm{C}\left(6^{\prime}\right)-\mathrm{Fe}\left(1^{\prime}\right)$ & $69.6(2)$ \\
\hline $\mathrm{C}\left(3^{\prime}\right)-\mathrm{Fe}\left(1^{\prime}\right)-\mathrm{C}\left(9^{\prime}\right)$ & $140.82(16)$ & $\mathrm{P}\left(2^{\prime}\right)-\mathrm{C}\left(6^{\prime}\right)-\mathrm{Fe}\left(1^{\prime}\right)$ & $124.37(18)$ \\
\hline$C\left(1^{\prime}\right)-P\left(1^{\prime}\right)-C\left(17^{\prime}\right)$ & 101.89(16) & $C\left(8^{\prime}\right)-C\left(7^{\prime}\right)-C\left(6^{\prime}\right)$ & $107.2(3)$ \\
\hline$C\left(1^{\prime}\right)-P\left(1^{\prime}\right)-C\left(11^{\prime}\right)$ & $102.24(16)$ & $\mathrm{C}\left(8^{\prime}\right)-\mathrm{C}\left(7^{\prime}\right)-\mathrm{Fe}\left(1^{\prime}\right)$ & $70.7(2)$ \\
\hline $\mathrm{C}\left(17^{\prime}\right)-\mathrm{P}\left(1^{\prime}\right)-\mathrm{C}\left(11^{\prime}\right)$ & 107.01(16) & $\mathrm{C}\left(6^{\prime}\right)-\mathrm{C}\left(7^{\prime}\right)-\mathrm{Fe}\left(1^{\prime}\right)$ & $68.70(19)$ \\
\hline $\mathrm{C}\left(1^{\prime}\right)-\mathrm{P}\left(1^{\prime}\right)-\mathrm{Pd}\left(1^{\prime}\right)$ & $120.18(11)$ & $\mathrm{C}\left(9^{\prime}\right)-\mathrm{C}\left(8^{\prime}\right)-\mathrm{C}\left(7^{\prime}\right)$ & $109.4(3)$ \\
\hline$C\left(17^{\prime}\right)-P\left(1^{\prime}\right)-\operatorname{Pd}\left(1^{\prime}\right)$ & $109.71(12)$ & $\mathrm{C}\left(9^{\prime}\right)-\mathrm{C}\left(8^{\prime}\right)-\mathrm{Fe}\left(1^{\prime}\right)$ & $70.3(2)$ \\
\hline$C\left(11^{\prime}\right)-P\left(1^{\prime}\right)-P d\left(1^{\prime}\right)$ & $114.43(13)$ & $\mathrm{C}\left(7^{\prime}\right)-\mathrm{C}\left(8^{\prime}\right)-\mathrm{Fe}\left(1^{\prime}\right)$ & $68.8(2)$ \\
\hline $\mathrm{C}\left(6^{\prime}\right)-\mathrm{P}\left(2^{\prime}\right)-\mathrm{C}\left(23^{\prime}\right)$ & $110.35(17)$ & $\mathrm{C}\left(8^{\prime}\right)-\mathrm{C}\left(9^{\prime}\right)-\mathrm{C}\left(10^{\prime}\right)$ & $107.7(3)$ \\
\hline $\mathrm{C}\left(6^{\prime}\right)-\mathrm{P}\left(2^{\prime}\right)-\mathrm{C}\left(29^{\prime}\right)$ & $103.21(17)$ & $\mathrm{C}\left(8^{\prime}\right)-\mathrm{C}\left(9^{\prime}\right)-\mathrm{Fe}\left(1^{\prime}\right)$ & $69.8(2)$ \\
\hline $\mathrm{C}\left(23^{\prime}\right)-\mathrm{P}\left(2^{\prime}\right)-\mathrm{C}\left(29^{\prime}\right)$ & $103.54(17)$ & $\mathrm{C}\left(10^{\prime}\right)-\mathrm{C}\left(9^{\prime}\right)-\mathrm{Fe}\left(1^{\prime}\right)$ & $68.9(2)$ \\
\hline $\mathrm{C}\left(6^{\prime}\right)-\mathrm{P}\left(2^{\prime}\right)-\mathrm{Pd}\left(1^{\prime}\right)$ & $111.42(12)$ & $\mathrm{C}\left(9^{\prime}\right)-\mathrm{C}\left(10^{\prime}\right)-\mathrm{C}\left(6^{\prime}\right)$ & $108.6(3)$ \\
\hline $\mathrm{C}\left(23^{\prime}\right)-\mathrm{P}\left(2^{\prime}\right)-\mathrm{Pd}\left(1^{\prime}\right)$ & $111.55(13)$ & $\mathrm{C}\left(9^{\prime}\right)-\mathrm{C}\left(10^{\prime}\right)-\mathrm{Fe}\left(1^{\prime}\right)$ & $70.6(2)$ \\
\hline $\mathrm{C}\left(29^{\prime}\right)-\mathrm{P}\left(2^{\prime}\right)-\mathrm{Pd}\left(1^{\prime}\right)$ & $116.21(12)$ & $\mathrm{C}\left(6^{\prime}\right)-\mathrm{C}\left(10^{\prime}\right)-\mathrm{Fe}\left(1^{\prime}\right)$ & $68.63(19)$ \\
\hline $\mathrm{C}\left(2^{\prime}\right)-\mathrm{C}\left(1^{\prime}\right)-\mathrm{C}\left(5^{\prime}\right)$ & $106.9(3)$ & $\mathrm{C}\left(16^{\prime}\right)-\mathrm{C}\left(11^{\prime}\right)-\mathrm{C}\left(12^{\prime}\right)$ & $118.9(4)$ \\
\hline $\mathrm{C}\left(2^{\prime}\right)-\mathrm{C}\left(1^{\prime}\right)-\mathrm{P}\left(1^{\prime}\right)$ & $124.6(3)$ & $\mathrm{C}\left(16^{\prime}\right)-\mathrm{C}\left(11^{\prime}\right)-\mathrm{P}\left(1^{\prime}\right)$ & $119.6(3)$ \\
\hline$C\left(5^{\prime}\right)-C\left(1^{\prime}\right)-P\left(1^{\prime}\right)$ & $128.5(3)$ & $\mathrm{C}\left(12^{\prime}\right)-\mathrm{C}\left(11^{\prime}\right)-\mathrm{P}\left(1^{\prime}\right)$ & $121.3(3)$ \\
\hline$C\left(2^{\prime}\right)-C\left(1^{\prime}\right)-\mathrm{Fe}\left(1^{\prime}\right)$ & 69.61(19) & $\mathrm{C}\left(13^{\prime}\right)-\mathrm{C}\left(12^{\prime}\right)-\mathrm{C}\left(11^{\prime}\right)$ & $120.0(4)$ \\
\hline $\mathrm{C}\left(5^{\prime}\right)-\mathrm{C}\left(1^{\prime}\right)-\mathrm{Fe}\left(1^{\prime}\right)$ & $69.92(19)$ & $\mathrm{C}\left(14^{\prime}\right)-\mathrm{C}\left(13^{\prime}\right)-\mathrm{C}\left(12^{\prime}\right)$ & $120.4(4)$ \\
\hline$P\left(1^{\prime}\right)-C\left(1^{\prime}\right)-F e\left(1^{\prime}\right)$ & $123.96(18)$ & $\mathrm{C}\left(13^{\prime}\right)-\mathrm{C}\left(14^{\prime}\right)-\mathrm{C}\left(15^{\prime}\right)$ & $120.4(4)$ \\
\hline $\mathrm{C}\left(3^{\prime}\right)-\mathrm{C}\left(2^{\prime}\right)-\mathrm{C}\left(1^{\prime}\right)$ & $108.2(3)$ & $\mathrm{C}\left(14^{\prime}\right)-\mathrm{C}\left(15^{\prime}\right)-\mathrm{C}\left(16^{\prime}\right)$ & $119.5(4)$ \\
\hline $\mathrm{C}\left(3^{\prime}\right)-\mathrm{C}\left(2^{\prime}\right)-\mathrm{Fe}\left(1^{\prime}\right)$ & $70.6(2)$ & $\mathrm{C}\left(11^{\prime}\right)-\mathrm{C}\left(16^{\prime}\right)-\mathrm{C}\left(15^{\prime}\right)$ & $120.8(4)$ \\
\hline$C\left(1^{\prime}\right)-\mathrm{C}\left(2^{\prime}\right)-\mathrm{Fe}\left(1^{\prime}\right)$ & $69.3(2)$ & $\mathrm{C}\left(22^{\prime}\right)-\mathrm{C}\left(17^{\prime}\right)-\mathrm{C}\left(18^{\prime}\right)$ & $118.5(3)$ \\
\hline $\mathrm{C}\left(4^{\prime}\right)-\mathrm{C}\left(3^{\prime}\right)-\mathrm{C}\left(2^{\prime}\right)$ & $108.2(3)$ & $\mathrm{C}\left(22^{\prime}\right)-\mathrm{C}\left(17^{\prime}\right)-\mathrm{P}\left(1^{\prime}\right)$ & $117.3(3)$ \\
\hline$C\left(4^{\prime}\right)-C\left(3^{\prime}\right)-F e\left(1^{\prime}\right)$ & $70.2(2)$ & $\mathrm{C}\left(18^{\prime}\right)-\mathrm{C}\left(17^{\prime}\right)-\mathrm{P}\left(1^{\prime}\right)$ & $124.3(3)$ \\
\hline $\mathrm{C}\left(2^{\prime}\right)-\mathrm{C}\left(3^{\prime}\right)-\mathrm{Fe}\left(1^{\prime}\right)$ & $68.6(2)$ & $\mathrm{C}\left(19^{\prime}\right)-\mathrm{C}\left(18^{\prime}\right)-\mathrm{C}\left(17^{\prime}\right)$ & $121.3(3)$ \\
\hline$C\left(3^{\prime}\right)-C\left(4^{\prime}\right)-C\left(5^{\prime}\right)$ & $108.8(3)$ & $\mathrm{C}\left(20^{\prime}\right)-\mathrm{C}\left(19^{\prime}\right)-\mathrm{C}\left(18^{\prime}\right)$ & $120.1(3)$ \\
\hline $\mathrm{C}\left(3^{\prime}\right)-\mathrm{C}\left(4^{\prime}\right)-\mathrm{Fe}\left(1^{\prime}\right)$ & $70.0(2)$ & $\mathrm{C}\left(19^{\prime}\right)-\mathrm{C}\left(20^{\prime}\right)-\mathrm{C}\left(21^{\prime}\right)$ & $119.4(4)$ \\
\hline $\mathrm{C}\left(5^{\prime}\right)-\mathrm{C}\left(4^{\prime}\right)-\mathrm{Fe}\left(1^{\prime}\right)$ & $69.2(2)$ & $\mathrm{C}\left(22^{\prime}\right)-\mathrm{C}\left(21^{\prime}\right)-\mathrm{C}\left(20^{\prime}\right)$ & $120.3(4)$ \\
\hline
\end{tabular}




\begin{tabular}{|c|c|c|c|}
\hline $\mathrm{C}\left(17^{\prime}\right)-\mathrm{C}\left(22^{\prime}\right)-\mathrm{C}\left(21^{\prime}\right)$ & $120.3(3)$ & $\mathrm{C}\left(38^{\prime}\right)-\mathrm{C}\left(39^{\prime}\right)-\mathrm{C}\left(40^{\prime}\right)$ & $120.7(4)$ \\
\hline$C\left(28^{\prime}\right)-C\left(23^{\prime}\right)-C\left(24^{\prime}\right)$ & $118.9(3)$ & $\mathrm{C}\left(41^{\prime}\right)-\mathrm{C}\left(40^{\prime}\right)-\mathrm{C}\left(39^{\prime}\right)$ & $120.7(4)$ \\
\hline $\mathrm{C}\left(28^{\prime}\right)-\mathrm{C}\left(23^{\prime}\right)-\mathrm{P}\left(2^{\prime}\right)$ & $119.0(3)$ & $\mathrm{C}\left(40^{\prime}\right)-\mathrm{C}\left(41^{\prime}\right)-\mathrm{C}\left(36^{\prime}\right)$ & $120.3(4)$ \\
\hline $\mathrm{C}\left(24^{\prime}\right)-\mathrm{C}\left(23^{\prime}\right)-\mathrm{P}\left(2^{\prime}\right)$ & $121.9(3)$ & $\mathrm{O}(1)-\mathrm{S}(1)-\mathrm{O}(3)$ & $115.4(2)$ \\
\hline $\mathrm{C}\left(25^{\prime}\right)-\mathrm{C}\left(24^{\prime}\right)-\mathrm{C}\left(23^{\prime}\right)$ & $119.5(4)$ & $\mathrm{O}(1)-\mathrm{S}(1)-\mathrm{O}(2)$ & $114.8(2)$ \\
\hline$C\left(26^{\prime}\right)-C\left(25^{\prime}\right)-C\left(24^{\prime}\right)$ & $120.9(4)$ & $\mathrm{O}(3)-\mathrm{S}(1)-\mathrm{O}(2)$ & $114.94(19)$ \\
\hline $\mathrm{C}\left(25^{\prime}\right)-\mathrm{C}\left(26^{\prime}\right)-\mathrm{C}\left(27^{\prime}\right)$ & $120.1(4)$ & $\mathrm{O}(1)-\mathrm{S}(1)-\mathrm{C}(42)$ & $103.9(2)$ \\
\hline$C\left(26^{\prime}\right)-C\left(27^{\prime}\right)-C\left(28^{\prime}\right)$ & $119.3(4)$ & $\mathrm{O}(3)-\mathrm{S}(1)-\mathrm{C}(42)$ & $103.5(2)$ \\
\hline $\mathrm{C}\left(27^{\prime}\right)-\mathrm{C}\left(28^{\prime}\right)-\mathrm{C}\left(23^{\prime}\right)$ & $121.3(4)$ & $\mathrm{O}(2)-\mathrm{S}(1)-\mathrm{C}(42)$ & $101.8(2)$ \\
\hline$C\left(34^{\prime}\right)-C\left(29^{\prime}\right)-C\left(30^{\prime}\right)$ & $118.9(4)$ & $\mathrm{F}(1)-\mathrm{C}(42)-\mathrm{F}(2)$ & $106.5(4)$ \\
\hline $\mathrm{C}\left(34^{\prime}\right)-\mathrm{C}\left(29^{\prime}\right)-\mathrm{P}\left(2^{\prime}\right)$ & $119.2(3)$ & $\mathrm{F}(1)-\mathrm{C}(42)-\mathrm{F}(3)$ & $106.0(4)$ \\
\hline $\mathrm{C}\left(30^{\prime}\right)-\mathrm{C}\left(29^{\prime}\right)-\mathrm{P}\left(2^{\prime}\right)$ & $121.7(3)$ & $\mathrm{F}(2)-\mathrm{C}(42)-\mathrm{F}(3)$ & $106.5(4)$ \\
\hline $\mathrm{C}\left(31^{\prime}\right)-\mathrm{C}\left(30^{\prime}\right)-\mathrm{C}\left(29^{\prime}\right)$ & $119.5(4)$ & $\mathrm{F}(1)-\mathrm{C}(42)-\mathrm{S}(1)$ & $113.4(3)$ \\
\hline$C\left(32^{\prime}\right)-C\left(31^{\prime}\right)-C\left(30^{\prime}\right)$ & $120.8(5)$ & $\mathrm{F}(2)-\mathrm{C}(42)-\mathrm{S}(1)$ & $112.2(3)$ \\
\hline $\mathrm{C}\left(31^{\prime}\right)-\mathrm{C}\left(32^{\prime}\right)-\mathrm{C}\left(33^{\prime}\right)$ & $120.4(4)$ & $\mathrm{F}(3)-\mathrm{C}(42)-\mathrm{S}(1)$ & $111.7(3)$ \\
\hline$C\left(32^{\prime}\right)-C\left(33^{\prime}\right)-C\left(34^{\prime}\right)$ & $119.5(4)$ & $\mathrm{O}\left(3^{\prime}\right)-\mathrm{S}\left(1^{\prime}\right)-\mathrm{O}\left(2^{\prime}\right)$ & $114.9(2)$ \\
\hline $\mathrm{C}\left(29^{\prime}\right)-\mathrm{C}\left(34^{\prime}\right)-\mathrm{C}\left(33^{\prime}\right)$ & $120.9(4)$ & $\mathrm{O}\left(3^{\prime}\right)-\mathrm{S}\left(1^{\prime}\right)-\mathrm{O}\left(1^{\prime}\right)$ & $114.51(19)$ \\
\hline $\mathrm{H}(35 \mathrm{D})-\mathrm{C}\left(35^{\prime}\right)-\mathrm{H}(35 \mathrm{C})$ & 113(3) & $\mathrm{O}\left(2^{\prime}\right)-\mathrm{S}\left(1^{\prime}\right)-\mathrm{O}\left(1^{\prime}\right)$ & $116.19(18)$ \\
\hline $\mathrm{H}(35 \mathrm{D})-\mathrm{C}\left(35^{\prime}\right)-\mathrm{C}\left(36^{\prime}\right)$ & $119(2)$ & $\mathrm{O}\left(3^{\prime}\right)-\mathrm{S}\left(1^{\prime}\right)-\mathrm{C}\left(42^{\prime}\right)$ & $102.57(19)$ \\
\hline $\mathrm{H}(35 \mathrm{C})-\mathrm{C}\left(35^{\prime}\right)-\mathrm{C}\left(36^{\prime}\right)$ & $119(2)$ & $\mathrm{O}\left(2^{\prime}\right)-\mathrm{S}\left(1^{\prime}\right)-\mathrm{C}\left(42^{\prime}\right)$ & $102.75(19)$ \\
\hline $\mathrm{H}(35 \mathrm{D})-\mathrm{C}\left(35^{\prime}\right)-\mathrm{Pd}\left(1^{\prime}\right)$ & $121(2)$ & $\mathrm{O}\left(1^{\prime}\right)-\mathrm{S}\left(1^{\prime}\right)-\mathrm{C}\left(42^{\prime}\right)$ & $103.29(19)$ \\
\hline $\mathrm{H}(35 \mathrm{C})-\mathrm{C}\left(35^{\prime}\right)-\mathrm{Pd}\left(1^{\prime}\right)$ & $104(2)$ & $\mathrm{F}\left(1^{\prime}\right)-\mathrm{C}\left(42^{\prime}\right)-\mathrm{F}\left(2^{\prime}\right)$ & $107.0(3)$ \\
\hline $\mathrm{C}\left(36^{\prime}\right)-\mathrm{C}\left(35^{\prime}\right)-\mathrm{Pd}\left(1^{\prime}\right)$ & $75.9(2)$ & $\mathrm{F}\left(1^{\prime}\right)-\mathrm{C}\left(42^{\prime}\right)-\mathrm{F}\left(3^{\prime}\right)$ & $107.3(3)$ \\
\hline $\mathrm{C}\left(37^{\prime}\right)-\mathrm{C}\left(36^{\prime}\right)-\mathrm{C}\left(41^{\prime}\right)$ & $117.4(4)$ & $\mathrm{F}\left(2^{\prime}\right)-\mathrm{C}\left(42^{\prime}\right)-\mathrm{F}\left(3^{\prime}\right)$ & $106.6(3)$ \\
\hline $\mathrm{C}\left(37^{\prime}\right)-\mathrm{C}\left(36^{\prime}\right)-\mathrm{C}\left(35^{\prime}\right)$ & $118.1(4)$ & $\mathrm{F}\left(1^{\prime}\right)-\mathrm{C}\left(42^{\prime}\right)-\mathrm{S}\left(1^{\prime}\right)$ & $112.2(3)$ \\
\hline $\mathrm{C}\left(41^{\prime}\right)-\mathrm{C}\left(36^{\prime}\right)-\mathrm{C}\left(35^{\prime}\right)$ & $123.5(4)$ & $\mathrm{F}\left(2^{\prime}\right)-\mathrm{C}\left(42^{\prime}\right)-\mathrm{S}\left(1^{\prime}\right)$ & $112.0(3)$ \\
\hline $\mathrm{C}\left(37^{\prime}\right)-\mathrm{C}\left(36^{\prime}\right)-\mathrm{Pd}\left(1^{\prime}\right)$ & $77.5(2)$ & $\mathrm{F}\left(3^{\prime}\right)-\mathrm{C}\left(42^{\prime}\right)-\mathrm{S}\left(1^{\prime}\right)$ & $111.4(3)$ \\
\hline $\mathrm{C}\left(41^{\prime}\right)-\mathrm{C}\left(36^{\prime}\right)-\mathrm{Pd}\left(1^{\prime}\right)$ & $118.3(3)$ & $\mathrm{Cl}(1)-\mathrm{C}(43)-\mathrm{Cl}(2)$ & $112.4(3)$ \\
\hline $\mathrm{C}\left(35^{\prime}\right)-\mathrm{C}\left(36^{\prime}\right)-\mathrm{Pd}\left(1^{\prime}\right)$ & $66.0(2)$ & & \\
\hline $\mathrm{H}(37 \mathrm{~B})-\mathrm{C}\left(37^{\prime}\right)-\mathrm{C}\left(38^{\prime}\right)$ & $122(2)$ & & \\
\hline $\mathrm{H}(37 \mathrm{~B})-\mathrm{C}\left(37^{\prime}\right)-\mathrm{C}\left(36^{\prime}\right)$ & $115.6(19)$ & & \\
\hline $\mathrm{C}\left(38^{\prime}\right)-\mathrm{C}\left(37^{\prime}\right)-\mathrm{C}\left(36^{\prime}\right)$ & $120.8(4)$ & & \\
\hline $\mathrm{H}(37 \mathrm{~B})-\mathrm{C}\left(37^{\prime}\right)-\mathrm{Pd}\left(1^{\prime}\right)$ & 86.1(19) & & \\
\hline $\mathrm{C}\left(38^{\prime}\right)-\mathrm{C}\left(37^{\prime}\right)-\mathrm{Pd}\left(1^{\prime}\right)$ & $124.8(3)$ & & \\
\hline $\mathrm{C}\left(36^{\prime}\right)-\mathrm{C}\left(37^{\prime}\right)-\mathrm{Pd}\left(1^{\prime}\right)$ & $67.1(2)$ & & \\
\hline $\mathrm{C}\left(39^{\prime}\right)-\mathrm{C}\left(38^{\prime}\right)-\mathrm{C}\left(37^{\prime}\right)$ & $120.0(4)$ & & \\
\hline
\end{tabular}


Table S19. Anisotropic displacement parameters $\left(\AA^{2} \times 10^{3}\right)$ for 9. The anisotropic displacement factor exponent takes the form: $-2 \pi^{2}\left[\mathrm{~h}^{2} \mathrm{a}^{* 2} \mathrm{U}^{11}+\ldots+2 \mathrm{hk} \mathrm{a} \mathrm{b}^{*} \mathrm{U}^{12}\right]$

\begin{tabular}{|c|c|c|c|c|c|c|}
\hline & $\mathrm{U}^{11}$ & $\mathrm{U}^{22}$ & $\mathrm{U}^{33}$ & $\mathrm{U}^{23}$ & $\mathrm{U}^{13}$ & $\mathrm{U}^{12}$ \\
\hline $\operatorname{Pd}(1)$ & $21(1)$ & $19(1)$ & $16(1)$ & $-7(1)$ & $0(1)$ & $-2(1)$ \\
\hline $\mathrm{Fe}(1)$ & $18(1)$ & $23(1)$ & $21(1)$ & $-10(1)$ & $0(1)$ & $-2(1)$ \\
\hline $\mathrm{P}(1)$ & $19(1)$ & $19(1)$ & $17(1)$ & $-7(1)$ & $-2(1)$ & $-1(1)$ \\
\hline $\mathrm{P}(2)$ & $22(1)$ & $18(1)$ & $21(1)$ & $-7(1)$ & $-1(1)$ & $-1(1)$ \\
\hline $\mathrm{C}(1)$ & $17(2)$ & $23(2)$ & $17(2)$ & $-8(2)$ & $0(2)$ & $2(2)$ \\
\hline $\mathrm{C}(2)$ & $19(2)$ & $24(2)$ & $20(2)$ & $-8(2)$ & $-2(2)$ & $-2(2)$ \\
\hline$C(3)$ & $29(2)$ & $39(2)$ & $19(2)$ & $-15(2)$ & $2(2)$ & $-4(2)$ \\
\hline$C(4)$ & $29(2)$ & $33(2)$ & $20(2)$ & $-9(2)$ & $2(2)$ & $-2(2)$ \\
\hline$C(5)$ & $28(2)$ & $20(2)$ & $21(2)$ & $-7(2)$ & $-1(2)$ & $-4(2)$ \\
\hline$C(6)$ & $20(2)$ & $18(2)$ & $25(2)$ & $-8(2)$ & $-3(2)$ & $0(2)$ \\
\hline$C(7)$ & $29(2)$ & $25(2)$ & $33(2)$ & $-15(2)$ & $-1(2)$ & $5(2)$ \\
\hline$C(8)$ & $24(2)$ & $30(2)$ & $38(3)$ & $-11(2)$ & $1(2)$ & $6(2)$ \\
\hline $\mathrm{C}(9)$ & $19(2)$ & $30(2)$ & $41(3)$ & $-8(2)$ & $-9(2)$ & $0(2)$ \\
\hline$C(10)$ & $23(2)$ & $28(2)$ & $22(2)$ & $-7(2)$ & $-7(2)$ & $-1(2)$ \\
\hline $\mathrm{C}(11)$ & $19(2)$ & $18(2)$ & $20(2)$ & $-9(2)$ & $2(2)$ & $-3(2)$ \\
\hline$C(12)$ & $22(2)$ & $35(2)$ & $29(2)$ & $-9(2)$ & $-4(2)$ & $-1(2)$ \\
\hline$C(13)$ & $40(3)$ & $40(3)$ & $26(2)$ & $-8(2)$ & $-12(2)$ & $-1(2)$ \\
\hline$C(14)$ & $33(3)$ & $38(3)$ & $45(3)$ & $-13(2)$ & $-18(2)$ & $-5(2)$ \\
\hline$C(15)$ & $18(2)$ & $34(2)$ & $45(3)$ & $-13(2)$ & $1(2)$ & $-7(2)$ \\
\hline$C(16)$ & $26(2)$ & $26(2)$ & $31(2)$ & $-11(2)$ & $-3(2)$ & $-3(2)$ \\
\hline$C(17)$ & $21(2)$ & $19(2)$ & $18(2)$ & $-8(2)$ & $2(2)$ & $-4(2)$ \\
\hline$C(18)$ & $27(2)$ & $27(2)$ & $22(2)$ & $-12(2)$ & $-2(2)$ & $-2(2)$ \\
\hline$C(19)$ & $32(3)$ & $20(2)$ & $40(3)$ & $-12(2)$ & $-3(2)$ & $-2(2)$ \\
\hline$C(20)$ & $42(3)$ & $28(2)$ & $34(3)$ & $-16(2)$ & $3(2)$ & $-15(2)$ \\
\hline $\mathrm{C}(21)$ & $26(2)$ & $32(2)$ & $30(2)$ & $-15(2)$ & $-3(2)$ & $-9(2)$ \\
\hline$C(22)$ & $32(2)$ & $21(2)$ & $24(2)$ & $-9(2)$ & $-9(2)$ & $1(2)$ \\
\hline$C(23)$ & $24(2)$ & $20(2)$ & $18(2)$ & $-7(2)$ & $-2(2)$ & $-6(2)$ \\
\hline$C(24)$ & $33(3)$ & $23(2)$ & $35(2)$ & $-14(2)$ & $-1(2)$ & $-4(2)$ \\
\hline$C(25)$ & $48(3)$ & $26(2)$ & $40(3)$ & $-16(2)$ & $4(2)$ & $-16(2)$ \\
\hline$C(26)$ & $45(3)$ & $46(3)$ & $39(3)$ & $-22(2)$ & $1(2)$ & $-21(2)$ \\
\hline
\end{tabular}




\begin{tabular}{|c|c|c|c|c|c|c|}
\hline$C(27)$ & $27(3)$ & $45(3)$ & $52(3)$ & $-21(2)$ & $-6(2)$ & $-6(2)$ \\
\hline$C(28)$ & $27(2)$ & $29(2)$ & $38(3)$ & $-18(2)$ & $-5(2)$ & $-6(2)$ \\
\hline$C(29)$ & $30(2)$ & $19(2)$ & $22(2)$ & $-10(2)$ & $-3(2)$ & $2(2)$ \\
\hline$C(30)$ & $37(3)$ & $25(2)$ & $23(2)$ & $-9(2)$ & $0(2)$ & $-5(2)$ \\
\hline$C(31)$ & $45(3)$ & $31(2)$ & $25(2)$ & $-11(2)$ & $4(2)$ & $-12(2)$ \\
\hline$C(32)$ & $54(3)$ & $23(2)$ & $19(2)$ & $0(2)$ & $-5(2)$ & $-6(2)$ \\
\hline$C(33)$ & $38(3)$ & $40(3)$ & $32(3)$ & $-9(2)$ & $-12(2)$ & $-2(2)$ \\
\hline$C(34)$ & $28(3)$ & $31(2)$ & $31(2)$ & $-7(2)$ & $-6(2)$ & $-6(2)$ \\
\hline$C(35)$ & $37(3)$ & $33(3)$ & $22(2)$ & $-9(2)$ & $-2(2)$ & $-5(2)$ \\
\hline$C(36)$ & $29(2)$ & $26(2)$ & $13(2)$ & $-11(2)$ & $2(2)$ & $-5(2)$ \\
\hline$C(37)$ & $30(3)$ & $32(2)$ & $20(2)$ & $-16(2)$ & $3(2)$ & $-7(2)$ \\
\hline$C(38)$ & $34(3)$ & $32(2)$ & $23(2)$ & $-8(2)$ & $0(2)$ & $3(2)$ \\
\hline C(39) & $49(3)$ & $26(2)$ & $33(3)$ & $-15(2)$ & $2(2)$ & $-2(2)$ \\
\hline$C(40)$ & $41(3)$ & $45(3)$ & $33(3)$ & $-23(2)$ & $12(2)$ & $-22(2)$ \\
\hline$C(41)$ & $22(2)$ & $44(3)$ & $30(2)$ & $-20(2)$ & $1(2)$ & $-2(2)$ \\
\hline $\operatorname{Pd}\left(1^{\prime}\right)$ & $21(1)$ & $16(1)$ & $18(1)$ & $-7(1)$ & $0(1)$ & $-1(1)$ \\
\hline $\operatorname{Fe}\left(1^{\prime}\right)$ & $19(1)$ & $20(1)$ & $20(1)$ & $-10(1)$ & $-2(1)$ & $-1(1)$ \\
\hline$P\left(1^{\prime}\right)$ & $18(1)$ & $18(1)$ & $20(1)$ & $-7(1)$ & $-2(1)$ & $-1(1)$ \\
\hline$P\left(2^{\prime}\right)$ & $22(1)$ & $16(1)$ & $22(1)$ & $-7(1)$ & $-2(1)$ & $-2(1)$ \\
\hline$C\left(1^{\prime}\right)$ & $21(2)$ & $14(2)$ & $17(2)$ & $-7(2)$ & $-4(2)$ & $3(2)$ \\
\hline$C\left(2^{\prime}\right)$ & $19(2)$ & $24(2)$ & $28(2)$ & $-12(2)$ & $-6(2)$ & $-2(2)$ \\
\hline$C\left(3^{\prime}\right)$ & $39(3)$ & $36(2)$ & $24(2)$ & $-20(2)$ & $-12(2)$ & $6(2)$ \\
\hline$C\left(4^{\prime}\right)$ & $34(3)$ & $30(2)$ & $17(2)$ & $-5(2)$ & $4(2)$ & $1(2)$ \\
\hline$C\left(5^{\prime}\right)$ & $26(2)$ & $22(2)$ & $20(2)$ & $-7(2)$ & $0(2)$ & $-2(2)$ \\
\hline$C\left(6^{\prime}\right)$ & $21(2)$ & $15(2)$ & $20(2)$ & $-5(2)$ & $-4(2)$ & $2(2)$ \\
\hline$C\left(7^{\prime}\right)$ & $21(2)$ & $18(2)$ & $32(2)$ & $-10(2)$ & $-3(2)$ & $4(2)$ \\
\hline$C\left(8^{\prime}\right)$ & $18(2)$ & $27(2)$ & $30(2)$ & $-14(2)$ & $2(2)$ & $6(2)$ \\
\hline$C\left(9^{\prime}\right)$ & $15(2)$ & $31(2)$ & $32(2)$ & $-11(2)$ & $-4(2)$ & $-3(2)$ \\
\hline$C\left(10^{\prime}\right)$ & $18(2)$ & $24(2)$ & $21(2)$ & $-11(2)$ & $-4(2)$ & $-1(2)$ \\
\hline$C\left(11^{\prime}\right)$ & $23(2)$ & $18(2)$ & $24(2)$ & $-8(2)$ & $-2(2)$ & $-3(2)$ \\
\hline$C\left(12^{\prime}\right)$ & $25(2)$ & $32(2)$ & $28(2)$ & $-9(2)$ & $-3(2)$ & $-1(2)$ \\
\hline$C\left(13^{\prime}\right)$ & $38(3)$ & $43(3)$ & $38(3)$ & $-9(2)$ & $-21(2)$ & $2(2)$ \\
\hline$C\left(14^{\prime}\right)$ & $24(3)$ & $46(3)$ & $58(3)$ & $-16(3)$ & $-16(2)$ & $-4(2)$ \\
\hline$C\left(15^{\prime}\right)$ & $22(2)$ & $37(2)$ & $43(3)$ & $-15(2)$ & $-2(2)$ & $-5(2)$ \\
\hline$C\left(16^{\prime}\right)$ & $21(2)$ & $27(2)$ & $30(2)$ & $-11(2)$ & $-4(2)$ & $-3(2)$ \\
\hline$C\left(17^{\prime}\right)$ & $20(2)$ & $20(2)$ & $18(2)$ & $-11(2)$ & $2(2)$ & $-4(2)$ \\
\hline
\end{tabular}




\begin{tabular}{|c|c|c|c|c|c|c|}
\hline$C\left(18^{\prime}\right)$ & $18(2)$ & $24(2)$ & $26(2)$ & $-8(2)$ & $-1(2)$ & $-5(2)$ \\
\hline$C\left(19^{\prime}\right)$ & $36(3)$ & $17(2)$ & $33(2)$ & $-6(2)$ & $-7(2)$ & $1(2)$ \\
\hline $\mathrm{C}\left(20^{\prime}\right)$ & $37(3)$ & $23(2)$ & $43(3)$ & $-15(2)$ & $-1(2)$ & $-10(2)$ \\
\hline$C\left(21^{\prime}\right)$ & $30(2)$ & $32(2)$ & $44(3)$ & $-23(2)$ & $-5(2)$ & $-7(2)$ \\
\hline$C\left(22^{\prime}\right)$ & $29(2)$ & $18(2)$ & $31(2)$ & $-10(2)$ & $-4(2)$ & $1(2)$ \\
\hline$C\left(23^{\prime}\right)$ & $26(2)$ & $22(2)$ & $20(2)$ & $-5(2)$ & $-2(2)$ & $-9(2)$ \\
\hline$C\left(24^{\prime}\right)$ & $33(3)$ & $31(2)$ & $41(3)$ & $-21(2)$ & $5(2)$ & $-12(2)$ \\
\hline$C\left(25^{\prime}\right)$ & $55(3)$ & $48(3)$ & $51(3)$ & $-33(3)$ & $16(3)$ & $-30(3)$ \\
\hline$C\left(26^{\prime}\right)$ & $47(3)$ & $65(3)$ & $37(3)$ & $-21(3)$ & $6(2)$ & $-38(3)$ \\
\hline$C\left(27^{\prime}\right)$ & $31(3)$ & $44(3)$ & $52(3)$ & $-15(2)$ & $-2(2)$ & $-15(2)$ \\
\hline $\mathrm{C}\left(28^{\prime}\right)$ & $33(3)$ & $27(2)$ & $44(3)$ & $-13(2)$ & $-5(2)$ & $-8(2)$ \\
\hline$C\left(29^{\prime}\right)$ & $33(3)$ & $17(2)$ & $21(2)$ & $-9(2)$ & $0(2)$ & $4(2)$ \\
\hline$C\left(30^{\prime}\right)$ & $62(3)$ & $33(2)$ & $31(3)$ & $-10(2)$ & $2(2)$ & $-15(2)$ \\
\hline$C\left(31^{\prime}\right)$ & $101(5)$ & $23(2)$ & $30(3)$ & $2(2)$ & $5(3)$ & $-15(3)$ \\
\hline$C\left(32^{\prime}\right)$ & $74(4)$ & $37(3)$ & $28(3)$ & $-4(2)$ & $-13(3)$ & $24(3)$ \\
\hline$C\left(33^{\prime}\right)$ & $41(3)$ & $52(3)$ & $37(3)$ & $-10(3)$ & $-14(2)$ & $10(3)$ \\
\hline$C\left(34^{\prime}\right)$ & $36(3)$ & $37(3)$ & $34(3)$ & $-4(2)$ & $-9(2)$ & $-1(2)$ \\
\hline$C\left(35^{\prime}\right)$ & $42(3)$ & $32(3)$ & $16(2)$ & $-6(2)$ & $3(2)$ & $0(2)$ \\
\hline$C\left(36^{\prime}\right)$ & $31(3)$ & $33(2)$ & $17(2)$ & $-13(2)$ & $2(2)$ & $1(2)$ \\
\hline$C\left(37^{\prime}\right)$ & $21(2)$ & $30(2)$ & $26(2)$ & $-18(2)$ & $3(2)$ & $-5(2)$ \\
\hline$C\left(38^{\prime}\right)$ & $34(3)$ & $25(2)$ & $27(2)$ & $-10(2)$ & $1(2)$ & $5(2)$ \\
\hline$C\left(39^{\prime}\right)$ & $52(3)$ & $33(2)$ & $44(3)$ & $-22(2)$ & $11(2)$ & $-14(2)$ \\
\hline$C\left(40^{\prime}\right)$ & $38(3)$ & $56(3)$ & $43(3)$ & $-31(3)$ & $10(2)$ & $-27(2)$ \\
\hline$C\left(41^{\prime}\right)$ & $19(2)$ & $53(3)$ & $31(2)$ & $-21(2)$ & $-1(2)$ & $1(2)$ \\
\hline $\mathrm{S}(1)$ & $42(1)$ & $54(1)$ & $33(1)$ & $-23(1)$ & $10(1)$ & $-23(1)$ \\
\hline $\mathrm{O}(1)$ & $91(3)$ & $68(2)$ & $49(2)$ & $-1(2)$ & $24(2)$ & $-31(2)$ \\
\hline $\mathrm{O}(2)$ & $77(3)$ & $97(3)$ & $55(2)$ & $-53(2)$ & $4(2)$ & $-42(2)$ \\
\hline $\mathrm{O}(3)$ & $33(2)$ & $84(2)$ & $63(2)$ & $-44(2)$ & $1(2)$ & $-7(2)$ \\
\hline$C(42)$ & $37(3)$ & $67(4)$ & $52(3)$ & $-33(3)$ & $-16(3)$ & $-2(3)$ \\
\hline $\mathrm{F}(1)$ & $59(2)$ & $112(2)$ & 107(3) & $-89(2)$ & $-34(2)$ & $14(2)$ \\
\hline $\mathrm{F}(2)$ & $100(3)$ & $55(2)$ & $98(3)$ & $-9(2)$ & $-31(2)$ & $-16(2)$ \\
\hline $\mathrm{F}(3)$ & $35(2)$ & $117(3)$ & $104(3)$ & $-67(2)$ & $-19(2)$ & $-2(2)$ \\
\hline$S\left(1^{\prime}\right)$ & $37(1)$ & $47(1)$ & $33(1)$ & $-19(1)$ & $2(1)$ & $-20(1)$ \\
\hline $\mathrm{O}\left(1^{\prime}\right)$ & $40(2)$ & $58(2)$ & $62(2)$ & $-40(2)$ & $7(2)$ & $-11(2)$ \\
\hline $\mathrm{O}\left(2^{\prime}\right)$ & $74(2)$ & $50(2)$ & $34(2)$ & $-15(2)$ & $-19(2)$ & $-10(2)$ \\
\hline $\mathrm{O}\left(3^{\prime}\right)$ & $59(2)$ & $97(3)$ & $53(2)$ & $-37(2)$ & $16(2)$ & $-55(2)$ \\
\hline
\end{tabular}




\begin{tabular}{lllllll}
$\mathrm{C}\left(42^{\prime}\right)$ & $33(3)$ & $40(3)$ & $38(3)$ & $-16(2)$ & $-5(2)$ & $-7(2)$ \\
$\mathrm{F}\left(1^{\prime}\right)$ & $47(2)$ & $60(2)$ & $36(2)$ & $-10(1)$ & $-13(1)$ & $6(1)$ \\
$\mathrm{F}\left(2^{\prime}\right)$ & $54(2)$ & $45(2)$ & $43(2)$ & $-25(1)$ & $-11(1)$ & $-3(1)$ \\
$\mathrm{F}\left(3^{\prime}\right)$ & $41(2)$ & $62(2)$ & $55(2)$ & $-24(2)$ & $12(1)$ & $-25(1)$ \\
$\mathrm{C}(43)$ & $45(3)$ & $58(3)$ & $50(3)$ & $-15(3)$ & $6(2)$ & $-16(3)$ \\
$\mathrm{Cl}(1)$ & $93(1)$ & $87(1)$ & $67(1)$ & $-39(1)$ & $-3(1)$ & $-30(1)$ \\
$\mathrm{Cl}(2)$ & $64(1)$ & $74(1)$ & $56(1)$ & $-30(1)$ & $-5(1)$ & $-5(1)$ \\
\hline
\end{tabular}


Table S20. Hydrogen coordinates $\left(x 1^{4}\right)$ and isotropic displacement parameters $\left(\AA^{2} x 1^{3}\right)$ for 9.

\begin{tabular}{|c|c|c|c|c|}
\hline & $\mathrm{x}$ & $\mathrm{y}$ & $\mathrm{z}$ & $\mathrm{U}(\mathrm{eq})$ \\
\hline $\mathrm{H}(2 \mathrm{~A})$ & -194 & 2456 & 9326 & 26 \\
\hline $\mathrm{H}(3 \mathrm{~A})$ & -2149 & 2384 & 10239 & 34 \\
\hline $\mathrm{H}(4 \mathrm{~A})$ & -3553 & 1471 & 10222 & 35 \\
\hline $\mathrm{H}(5 \mathrm{~A})$ & -2456 & 983 & 9294 & 29 \\
\hline $\mathrm{H}(7 \mathrm{~A})$ & -2692 & 3982 & 8634 & 35 \\
\hline $\mathrm{H}(8 \mathrm{~A})$ & -4818 & 3419 & 9173 & 40 \\
\hline $\mathrm{H}(9 \mathrm{~A})$ & -5099 & 2581 & 8586 & 39 \\
\hline $\mathrm{H}(10 \mathrm{~A})$ & -3160 & 2610 & 7666 & 31 \\
\hline $\mathrm{H}(12 \mathrm{~A})$ & 984 & 725 & 9870 & 37 \\
\hline $\mathrm{H}(13 \mathrm{~A})$ & 2971 & 422 & 10389 & 45 \\
\hline $\mathrm{H}(14 \mathrm{~A})$ & 4883 & 748 & 9696 & 47 \\
\hline$H(15 A)$ & 4832 & 1319 & 8474 & 41 \\
\hline $\mathrm{H}(16 \mathrm{~A})$ & 2836 & 1630 & 7952 & 34 \\
\hline $\mathrm{H}(18 \mathrm{~A})$ & 1209 & -95 & 8932 & 30 \\
\hline $\mathrm{H}(19 \mathrm{~A})$ & 571 & -1078 & 8813 & 37 \\
\hline $\mathrm{H}(20 \mathrm{~A})$ & -1318 & -856 & 8239 & 39 \\
\hline$H(21 A)$ & -2604 & 350 & 7784 & 34 \\
\hline $\mathrm{H}(22 \mathrm{~A})$ & -1906 & 1341 & 7851 & 32 \\
\hline $\mathrm{H}(24 \mathrm{~A})$ & -1219 & 4821 & 7749 & 36 \\
\hline$H(25 A)$ & 34 & 5209 & 8310 & 43 \\
\hline $\mathrm{H}(26 \mathrm{~A})$ & 1999 & 4439 & 8804 & 48 \\
\hline $\mathrm{H}(27 \mathrm{~A})$ & 2722 & 3313 & 8699 & 49 \\
\hline $\mathrm{H}(28 \mathrm{~A})$ & 1518 & 2945 & 8101 & 35 \\
\hline $\mathrm{H}(30 \mathrm{~A})$ & 320 & 4700 & 6382 & 35 \\
\hline $\mathrm{H}(31 \mathrm{~A})$ & -282 & 5712 & 5311 & 41 \\
\hline $\mathrm{H}(32 \mathrm{~A})$ & -2394 & 5966 & 4939 & 42 \\
\hline $\mathrm{H}(33 \mathrm{~A})$ & -3930 & 5222 & 5638 & 46 \\
\hline $\mathrm{H}(34 \mathrm{~A})$ & -3346 & 4195 & 6704 & 39 \\
\hline $\mathrm{H}(35 \mathrm{~A})$ & $660(30)$ & $3368(19)$ & $6076(18)$ & $27(12)$ \\
\hline $\mathrm{H}(35 \mathrm{~B})$ & $-810(40)$ & $3350(20)$ & $5970(20)$ & $51(13)$ \\
\hline
\end{tabular}




\begin{tabular}{|c|c|c|c|c|}
\hline $\mathrm{H}(37 \mathrm{~A})$ & $2260(30)$ & $2120(19)$ & $6682(18)$ & $35(11)$ \\
\hline $\mathrm{H}(38 \mathrm{~A})$ & 2686 & 830 & 7032 & 40 \\
\hline $\mathrm{H}(39 \mathrm{~A})$ & 1249 & 245 & 6803 & 44 \\
\hline $\mathrm{H}(40 \mathrm{~A})$ & -688 & 935 & 6255 & 44 \\
\hline $\mathrm{H}(41 \mathrm{~A})$ & -1278 & 2203 & 5983 & 37 \\
\hline $\mathrm{H}\left(2^{\prime} \mathrm{A}\right)$ & 5079 & 2604 & 3977 & 28 \\
\hline $\mathrm{H}\left(3^{\prime} \mathrm{A}\right)$ & 3267 & 2696 & 4929 & 37 \\
\hline $\mathrm{H}\left(4^{\prime} \mathrm{A}\right)$ & 1782 & 1730 & 5178 & 36 \\
\hline $\mathrm{H}\left(5^{\prime} \mathrm{A}\right)$ & 2647 & 1016 & 4398 & 29 \\
\hline $\mathrm{H}\left(7^{\prime} \mathrm{A}\right)$ & 2387 & 4083 & 3307 & 29 \\
\hline $\mathrm{H}\left(8^{\prime} \mathrm{A}\right)$ & 324 & 3504 & 3956 & 32 \\
\hline $\mathrm{H}\left(9^{\prime} \mathrm{A}\right)$ & 44 & 2522 & 3537 & 32 \\
\hline $\mathrm{H}(10 \mathrm{~B})$ & 1939 & 2478 & 2612 & 24 \\
\hline $\mathrm{H}(12 \mathrm{~B})$ & 6261 & 728 & 4698 & 36 \\
\hline $\mathrm{H}(13 \mathrm{~B})$ & 8377 & 438 & 5088 & 50 \\
\hline $\mathrm{H}(14 \mathrm{~B})$ & 10158 & 747 & 4286 & 52 \\
\hline $\mathrm{H}(15 \mathrm{~B})$ & 9858 & 1313 & 3082 & 42 \\
\hline $\mathrm{H}(16 \mathrm{~B})$ & 7743 & 1621 & 2684 & 31 \\
\hline $\mathrm{H}(18 \mathrm{~B})$ & 6104 & -162 & 3983 & 28 \\
\hline H(19B) & 5287 & -1215 & 4132 & 37 \\
\hline $\mathrm{H}(20 \mathrm{~B})$ & 3238 & -1073 & 3728 & 40 \\
\hline $\mathrm{H}(21 \mathrm{~B})$ & 2013 & 137 & 3169 & 38 \\
\hline $\mathrm{H}(22 \mathrm{~B})$ & 2899 & 1198 & 2953 & 32 \\
\hline $\mathrm{H}(24 \mathrm{~B})$ & 3913 & 4674 & 2605 & 39 \\
\hline $\mathrm{H}(25 \mathrm{~B})$ & 5480 & 5160 & 2882 & 55 \\
\hline $\mathrm{H}(26 \mathrm{~B})$ & 7689 & 4613 & 2904 & 55 \\
\hline $\mathrm{H}(27 \mathrm{~B})$ & 8367 & 3578 & 2630 & 51 \\
\hline $\mathrm{H}(28 \mathrm{~B})$ & 6809 & 3072 & 2374 & 42 \\
\hline $\mathrm{H}(30 \mathrm{~B})$ & 4946 & 4828 & 1169 & 51 \\
\hline $\mathrm{H}(31 \mathrm{~B})$ & 4171 & 5780 & 100 & 68 \\
\hline $\mathrm{H}(32 \mathrm{~B})$ & 2293 & 5736 & -330 & 66 \\
\hline $\mathrm{H}(33 \mathrm{~B})$ & 1153 & 4742 & 290 & 58 \\
\hline $\mathrm{H}(34 \mathrm{~B})$ & 1890 & 3793 & 1367 & 48 \\
\hline $\mathrm{H}(35 \mathrm{C})$ & $5880(30)$ & $3034(18)$ & $905(17)$ & $18(10)$ \\
\hline $\mathrm{H}(35 \mathrm{D})$ & $4400(40)$ & $3150(20)$ & $770(20)$ & $45(13)$ \\
\hline $\mathrm{H}(37 \mathrm{~B})$ & $6980(30)$ & $1762(16)$ & $1618(15)$ & $4(9)$ \\
\hline
\end{tabular}




\begin{tabular}{lrrrr}
$\mathrm{H}(38 \mathrm{~B})$ & 7142 & 444 & 2174 & 37 \\
$\mathrm{H}(39 \mathrm{~B})$ & 5539 & -66 & 1984 & 49 \\
$\mathrm{H}(40 \mathrm{~B})$ & 3750 & 711 & 1325 & 48 \\
$\mathrm{H}(41 \mathrm{~B})$ & 3521 & 2007 & 877 & 41 \\
$\mathrm{H}(43 \mathrm{~A})$ & -6088 & 6811 & 4964 & 64 \\
$\mathrm{H}(43 \mathrm{~B})$ & -6117 & 6408 & 5805 & 64 \\
\hline
\end{tabular}




\section{Experimental Procedure for the X-Ray Diffraction of 12}

\section{$\underline{\text { Data Collection }}$}

An orange blade crystal of $\mathrm{C}_{37} \mathrm{H}_{33} \mathrm{ClFeP}_{2} \mathrm{Pd} \cdot 3 \mathrm{CH}_{2} \mathrm{Cl}_{2}, \mathrm{C}_{40} \mathrm{H}_{39} \mathrm{Cl}_{7} \mathrm{FeP}_{2} \mathrm{Pd}$, having approximate dimensions of $0.25 \times 0.08 \times 0.08 \mathrm{~mm}^{3}$ was mounted with epoxy cement on the tip of a fine glass fiber. All measurements were made on a Nonius KappaCCD diffractometer with graphite monochromated Mo-K $\alpha$ radiation.

Cell constants and an orientation matrix for data collection corresponded to a primitive monoclinic cell with dimensions:

$$
\begin{array}{ll}
\mathrm{a}=9.5147(19) \AA & \alpha=90^{\circ} \\
\mathrm{b}=22.092(4) \AA & \beta=96.19(3) \mathrm{o} \\
\mathrm{c}=19.067(4) \AA & \gamma=90^{\circ} \\
\mathrm{V}=3984.5(14) \AA^{3} &
\end{array}
$$

For $Z=4$ and F.W. $=992.05$, the calculated density is $1.654 \mathrm{~g} / \mathrm{cm}^{3}$. Based on a statistical analysis of intensity distribution, and the successful solution and refinement of the structure, the space group was determined to be $P 2_{1} / n$ (\#14).

The data were collected at a temperature of 173(2) $\mathrm{K}$ to a maximum $2 \theta$ value of $56.50^{\circ}$. Four omega scans consisting of $75,75,75$, and 43 data frames, respectively, were collected with a frame width of $1.0^{\circ}$ and a detector-to-crystal distance, Dx, of $40.0 \mathrm{~mm}$. Each frame was exposed twice (for the purpose of de-zingering) for a total of $80 \mathrm{~s}$. The data frames were processed and scaled using the DENZO software package. ${ }^{1}$

\section{Data Reduction}

A total of 17456 reflections were collected of which 9801 were unique and observed $\left(\mathrm{R}_{\text {int }}\right.$ $=0.0395)$. The linear absorption coefficient, $\mu$, for Mo-K $\alpha$ radiation is $13.95 \mathrm{~cm}^{-1}$, and no absorption correction was applied. The data were corrected for Lorentz and polarization effects.

\section{$\underline{\text { Structure Solution and Refinement }}$}

The structure was solved by direct methods and expanded using Fourier techniques ${ }^{2}$. The non-hydrogen atoms were refined anisotropically, and hydrogen atoms were treated as idealized contributions. The final cycle of full-matrix least-squares refinement ${ }^{3}$ on $\mathrm{F}$ was based on 9801 observed reflections (I > 2.00 $(\mathrm{I})$ ) and 408 variable parameters and converged with unweighted and weighted agreement factors of:

$$
\begin{gathered}
\mathrm{R}=\Sigma\|\mathrm{Fo}|-| \mathrm{Fc}\| / \Sigma|\mathrm{Fo}|=0.0448 \\
\mathrm{R}_{\mathrm{W}}=\left\{\Sigma\left[\mathrm{w}\left(\mathrm{F}_{\mathrm{o}}^{2}-\mathrm{F}_{\mathrm{c}}^{2}\right)^{2}\right] / \Sigma\left[\mathrm{w}\left(\mathrm{F}_{\mathrm{o}}\right)^{2}\right]\right\}^{1 / 2}=0.1077
\end{gathered}
$$


The maximum and minimum peaks on the final difference Fourier map corresponded to 0.619 and $-0.849 \mathrm{e}^{-} / \AA^{3}$ respectively.

\section{REFERENCES}

(1) Z. Otwinowski and W. Minor, "Processing of X-Ray Diffraction Data Collected in Oscillation Mode," Methods in Enzymology, vol. 276: Macromolecular Crystallography, part A, 307-326, 1997, C.W. Carter, Jr. \& R.M. Sweet, Eds., Academic Press.

(2) Acta Cryst. A46 (1990) 467-473

(3) Least Squares function minimized:

$$
\Sigma w\left(\mathrm{~F}_{\mathrm{o}}{ }^{2}-\mathrm{F}_{\mathrm{c}}{ }^{2}\right)^{2}
$$




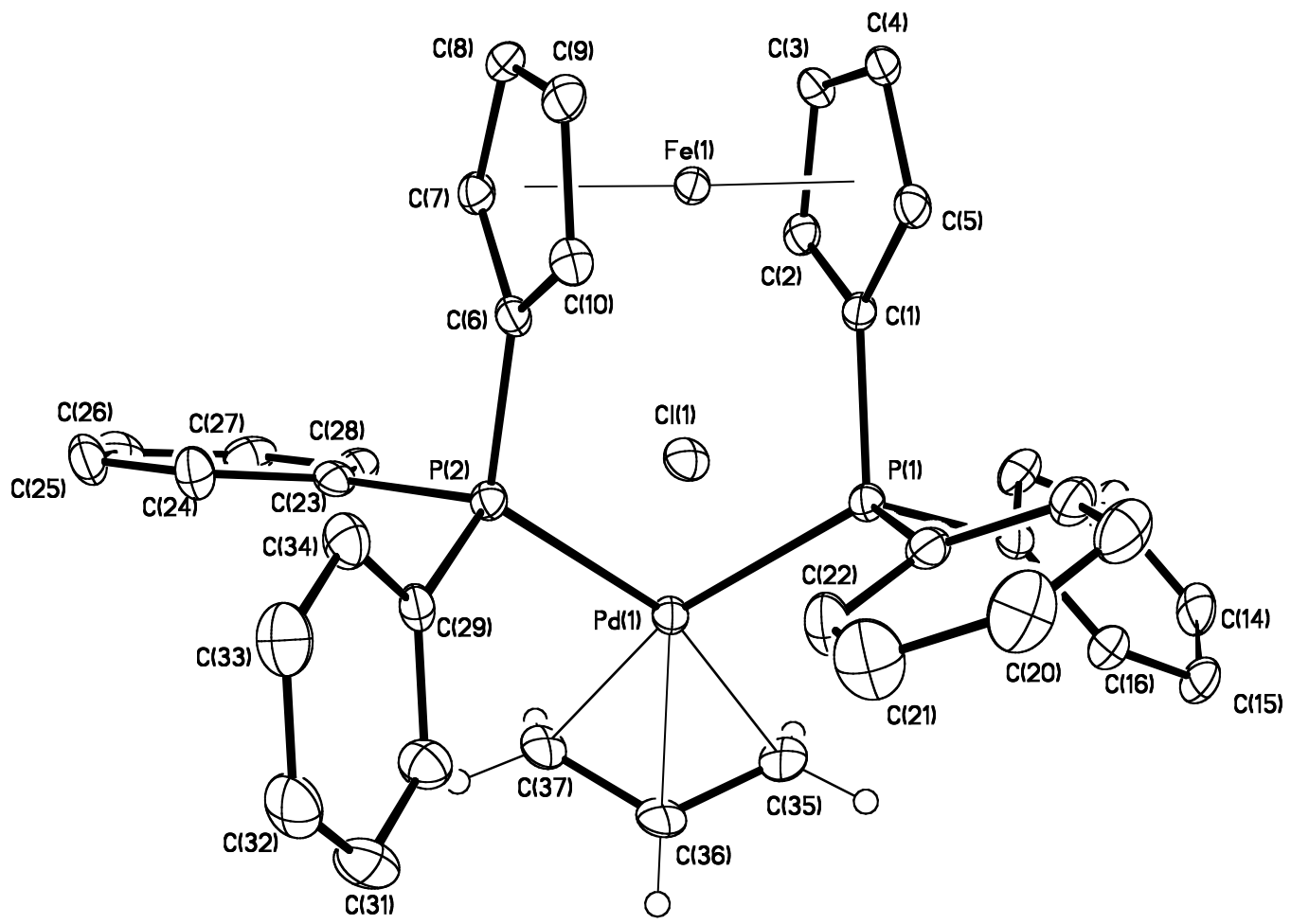

Figure S10. ORTEP diagram of 12 
Table S21. Crystal data and structure refinement for 12.

Empirical formula

Formula weight

Temperature

Wavelength

Crystal system

Space group

Unit cell dimensions

Volume

Z

Density (calculated)

Absorption coefficient

$\mathrm{F}(000)$

Crystal size

Theta range for data collection

Index ranges

Reflections collected

Independent reflections

Completeness to theta $=28.25^{\circ}$

Absorption correction

Max. and min. transmission

Refinement method

Data / restraints / parameters

Goodness-of-fit on $\mathrm{F}^{2}$

Final $\mathrm{R}$ indices [I $>2 \operatorname{sigma}(\mathrm{I})]$

$\mathrm{R}$ indices (all data)

Largest diff. peak and hole
$\mathrm{C}_{40} \mathrm{H}_{39} \mathrm{Cl}_{7} \mathrm{Fe} \mathrm{P} 2 \mathrm{Pd}$

992.05

173(2) K

$0.71073 \AA$

Monoclinic

$\mathrm{P} 2(1) / \mathrm{n}$

$\mathrm{a}=9.5147(19) \AA \quad \alpha=90^{\circ}$.

$\mathrm{b}=22.092(4) \AA \quad \beta=96.19(3)^{\circ}$.

$\mathrm{c}=19.067(4) \AA \quad \gamma=90^{\circ}$.

3984.5(14) $\AA^{3}$

4

$1.654 \mathrm{~g} / \mathrm{cm}^{3}$

$13.95 \mathrm{~cm}^{-1}$

2000

$0.25 \times 0.08 \times 0.08 \mathrm{~mm}^{3}$

2.15 to $28.25^{\circ}$.

$-12<=\mathrm{h}<=12,-26<=\mathrm{k}<=29,-25<=\mathrm{l}<=25$

17456

$9801[\mathrm{R}(\mathrm{int})=0.0395]$

$99.5 \%$

None

0.8966 and 0.7217

Full-matrix least-squares on $\mathrm{F}^{2}$

$9801 / 0 / 408$

1.009

$\mathrm{R} 1=0.0448, \mathrm{wR} 2=0.1077$

$\mathrm{R} 1=0.0745, \mathrm{wR} 2=0.1143$

0.619 and -0.849 e. $\AA^{-3}$ 
Table S22. Atomic coordinates ( $\times \mathbf{1 0}^{4}$ ) and equivalent isotropic displacement parameters $\left(\AA^{2} \mathbf{x} \mathbf{1 0}^{3}\right)$ for 12 . U(eq) is defined as one third of the trace of the orthogonalized Uij tensor.

\begin{tabular}{|c|c|c|c|c|}
\hline & $\mathrm{x}$ & $\mathrm{y}$ & $\mathrm{z}$ & $\mathrm{U}(\mathrm{eq})$ \\
\hline $\operatorname{Pd}(1)$ & $7322(1)$ & $2056(1)$ & $4335(1)$ & $24(1)$ \\
\hline $\mathrm{Fe}(1)$ & $3512(1)$ & $1078(1)$ & $4538(1)$ & $21(1)$ \\
\hline $\mathrm{Cl}(1)$ & $8123(1)$ & $816(1)$ & $3655(1)$ & $36(1)$ \\
\hline $\mathrm{P}(1)$ & $5220(1)$ & $2119(1)$ & $3618(1)$ & $23(1)$ \\
\hline $\mathrm{P}(2)$ & $6524(1)$ & $1763(1)$ & $5386(1)$ & $22(1)$ \\
\hline $\mathrm{C}(1)$ & $4041(3)$ & $1487(1)$ & $3645(2)$ & $22(1)$ \\
\hline$C(2)$ & $4442(3)$ & $866(2)$ & $3653(2)$ & $25(1)$ \\
\hline$C(3)$ & $3190(3)$ & $508(2)$ & $3674(2)$ & $25(1)$ \\
\hline$C(4)$ & 2011(3) & $910(2)$ & $3691(2)$ & $25(1)$ \\
\hline$C(5)$ & $2522(3)$ & $1507(2)$ & $3665(2)$ & $24(1)$ \\
\hline$C(6)$ & $4840(3)$ & $1376(1)$ & $5389(2)$ & $23(1)$ \\
\hline$C(7)$ & $4688(3)$ & $732(2)$ & $5415(2)$ & $25(1)$ \\
\hline $\mathrm{C}(8)$ & $3234(3)$ & $597(2)$ & $5437(2)$ & $28(1)$ \\
\hline$C(9)$ & $2482(3)$ & $1149(2)$ & $5423(2)$ & $30(1)$ \\
\hline$C(10)$ & $3458(3)$ & $1631(2)$ & $5383(2)$ & $25(1)$ \\
\hline$C(11)$ & $5511(3)$ & $2161(2)$ & $2685(2)$ & $26(1)$ \\
\hline$C(12)$ & $5827(4)$ & $1635(2)$ & $2344(2)$ & $35(1)$ \\
\hline$C(13)$ & $6243(4)$ & $1654(2)$ & $1664(2)$ & $42(1)$ \\
\hline$C(14)$ & $6346(4)$ & $2204(2)$ & $1337(2)$ & $41(1)$ \\
\hline$C(15)$ & $6028(4)$ & $2729(2)$ & $1669(2)$ & $39(1)$ \\
\hline$C(16)$ & $5618(4)$ & $2710(2)$ & $2342(2)$ & $34(1)$ \\
\hline$C(17)$ & $4112(3)$ & $2767(2)$ & $3777(2)$ & $28(1)$ \\
\hline$C(18)$ & $2927(3)$ & $2916(2)$ & $3309(2)$ & $35(1)$ \\
\hline$C(19)$ & $2050(4)$ & $3388(2)$ & $3476(2)$ & $45(1)$ \\
\hline$C(20)$ & $2330(5)$ & $3707(2)$ & $4094(2)$ & $55(1)$ \\
\hline$C(21)$ & $3504(5)$ & $3560(2)$ & $4553(3)$ & $61(1)$ \\
\hline$C(22)$ & $4385(4)$ & $3091(2)$ & $4401(2)$ & $40(1)$ \\
\hline$C(23)$ & $7714(3)$ & $1248(1)$ & $5916(2)$ & $25(1)$ \\
\hline$C(24)$ & $7808(4)$ & $1235(2)$ & $6648(2)$ & $33(1)$ \\
\hline$C(25)$ & $8726(4)$ & $852(2)$ & $7036(2)$ & $39(1)$ \\
\hline
\end{tabular}




\begin{tabular}{lrrrr}
$\mathrm{C}(26)$ & $9573(3)$ & $469(2)$ & $6703(2)$ & $38(1)$ \\
$\mathrm{C}(27)$ & $9485(3)$ & $463(2)$ & $5977(2)$ & $35(1)$ \\
$\mathrm{C}(28)$ & $8563(3)$ & $850(2)$ & $5576(2)$ & $30(1)$ \\
$\mathrm{C}(29)$ & $6386(3)$ & $2411(2)$ & $5973(2)$ & $26(1)$ \\
$\mathrm{C}(30)$ & $7325(4)$ & $2893(2)$ & $5936(2)$ & $36(1)$ \\
$\mathrm{C}(31)$ & $7293(4)$ & $3386(2)$ & $6371(2)$ & $46(1)$ \\
$\mathrm{C}(32)$ & $6301(4)$ & $3422(2)$ & $6855(2)$ & $46(1)$ \\
$\mathrm{C}(33)$ & $5367(4)$ & $2952(2)$ & $6894(2)$ & $44(1)$ \\
$\mathrm{C}(34)$ & $5398(4)$ & $2444(2)$ & $6468(2)$ & $34(1)$ \\
$\mathrm{C}(35)$ & $8625(3)$ & $2406(2)$ & $3553(2)$ & $37(1)$ \\
$\mathrm{C}(36)$ & $9271(3)$ & $2543(2)$ & $4218(2)$ & $36(1)$ \\
$\mathrm{C}(37)$ & $9566(3)$ & $2076(2)$ & $4707(2)$ & $33(1)$ \\
$\mathrm{C}(38)$ & $5917(5)$ & $-359(2)$ & $2735(2)$ & $60(1)$ \\
$\mathrm{Cl}(2)$ & $4927(2)$ & $-68(1)$ & $1988(1)$ & $87(1)$ \\
$\mathrm{Cl}(3)$ & $6651(1)$ & $-1064(1)$ & $2587(1)$ & $70(1)$ \\
& & & & \\
\hline
\end{tabular}


Table S23. Bond lengths $[\AA]$ and angles $\left[{ }^{\circ}\right]$ for 12 .

\begin{tabular}{|c|c|c|c|}
\hline $\operatorname{Pd}(1)-C(36)$ & $2.175(3)$ & $C(11)-C(16)$ & $1.385(5)$ \\
\hline $\operatorname{Pd}(1)-C(37)$ & $2.177(3)$ & $\mathrm{C}(12)-\mathrm{C}(13)$ & $1.396(5)$ \\
\hline $\operatorname{Pd}(1)-C(35)$ & $2.180(3)$ & $C(13)-C(14)$ & $1.373(5)$ \\
\hline $\mathrm{Pd}(1)-\mathrm{P}(1)$ & $2.3017(11)$ & $C(14)-C(15)$ & $1.370(5)$ \\
\hline $\operatorname{Pd}(1)-P(2)$ & $2.3109(10)$ & $C(15)-C(16)$ & $1.383(5)$ \\
\hline $\mathrm{Fe}(1)-\operatorname{cent}(1)$ & $1.717(2)$ & $C(17)-C(22)$ & $1.389(5)$ \\
\hline $\mathrm{Fe}(1)-\operatorname{cent}(2)$ & $1.714(2)$ & $\mathrm{C}(17)-\mathrm{C}(18)$ & $1.400(5)$ \\
\hline $\mathrm{Fe}(1)-\mathrm{C}(10)$ & $2.028(3)$ & $\mathrm{C}(18)-\mathrm{C}(19)$ & $1.393(5)$ \\
\hline $\mathrm{Fe}(1)-\mathrm{C}(1)$ & $2.039(3)$ & $C(19)-C(20)$ & $1.374(6)$ \\
\hline $\mathrm{Fe}(1)-\mathrm{C}(2)$ & $2.042(3)$ & $C(20)-C(21)$ & $1.382(6)$ \\
\hline $\mathrm{Fe}(1)-\mathrm{C}(9)$ & $2.045(3)$ & $C(21)-C(22)$ & $1.382(5)$ \\
\hline $\mathrm{Fe}(1)-\mathrm{C}(5)$ & $2.054(3)$ & $C(23)-C(24)$ & $1.389(5)$ \\
\hline $\mathrm{Fe}(1)-\mathrm{C}(6)$ & $2.055(3)$ & $\mathrm{C}(23)-\mathrm{C}(28)$ & $1.400(4)$ \\
\hline $\mathrm{Fe}(1)-\mathrm{C}(7)$ & $2.056(3)$ & $C(24)-C(25)$ & $1.373(5)$ \\
\hline $\mathrm{Fe}(1)-\mathrm{C}(8)$ & $2.057(3)$ & $C(25)-C(26)$ & $1.371(5)$ \\
\hline $\mathrm{Fe}(1)-\mathrm{C}(3)$ & $2.071(3)$ & $C(26)-C(27)$ & $1.377(5)$ \\
\hline $\mathrm{Fe}(1)-\mathrm{C}(4)$ & $2.071(3)$ & $\mathrm{C}(27)-\mathrm{C}(28)$ & $1.392(5)$ \\
\hline $\mathrm{P}(1)-\mathrm{C}(1)$ & $1.795(3)$ & $C(29)-C(30)$ & $1.396(5)$ \\
\hline$P(1)-C(17)$ & $1.823(3)$ & $C(29)-C(34)$ & $1.403(4)$ \\
\hline $\mathrm{P}(1)-\mathrm{C}(11)$ & $1.831(3)$ & $C(30)-C(31)$ & $1.372(5)$ \\
\hline $\mathrm{P}(2)-\mathrm{C}(6)$ & $1.817(3)$ & $C(31)-C(32)$ & $1.391(5)$ \\
\hline$P(2)-C(23)$ & $1.831(3)$ & $C(32)-C(33)$ & $1.372(5)$ \\
\hline $\mathrm{P}(2)-\mathrm{C}(29)$ & $1.831(3)$ & $C(33)-C(34)$ & $1.389(5)$ \\
\hline $\mathrm{C}(1)-\mathrm{C}(2)$ & $1.424(4)$ & $C(35)-C(36)$ & $1.381(5)$ \\
\hline$C(1)-C(5)$ & $1.451(4)$ & $C(36)-C(37)$ & $1.398(5)$ \\
\hline$C(2)-C(3)$ & $1.435(4)$ & $\mathrm{C}(38)-\mathrm{Cl}(2)$ & $1.744(5)$ \\
\hline$C(3)-C(4)$ & $1.434(4)$ & $\mathrm{C}(38)-\mathrm{Cl}(3)$ & $1.743(4)$ \\
\hline$C(4)-C(5)$ & $1.409(4)$ & & \\
\hline$C(6)-C(10)$ & $1.430(4)$ & $C(36)-P d(1)-C(37)$ & $37.48(13)$ \\
\hline$C(6)-C(7)$ & $1.431(4)$ & $C(36)-P d(1)-C(35)$ & $36.97(13)$ \\
\hline$C(7)-C(8)$ & $1.421(4)$ & $\mathrm{C}(37)-\mathrm{Pd}(1)-\mathrm{C}(35)$ & $66.76(13)$ \\
\hline $\mathrm{C}(8)-\mathrm{C}(9)$ & $1.413(5)$ & $\mathrm{C}(36)-\mathrm{Pd}(1)-\mathrm{P}(1)$ & $127.21(10)$ \\
\hline $\mathrm{C}(9)-\mathrm{C}(10)$ & $1.422(4)$ & $\mathrm{C}(37)-\mathrm{Pd}(1)-\mathrm{P}(1)$ & $162.05(10)$ \\
\hline$C(11)-C(12)$ & $1.382(5)$ & $\mathrm{C}(35)-\mathrm{Pd}(1)-\mathrm{P}(1)$ & $95.42(10)$ \\
\hline
\end{tabular}




\begin{tabular}{|c|c|c|c|}
\hline $\mathrm{C}(36)-\mathrm{Pd}(1)-\mathrm{P}(2)$ & $126.21(10)$ & $\mathrm{C}(9)-\mathrm{Fe}(1)-\mathrm{C}(3)$ & $131.27(13)$ \\
\hline$C(37)-P d(1)-P(2)$ & $97.22(10)$ & $\mathrm{C}(5)-\mathrm{Fe}(1)-\mathrm{C}(3)$ & $67.71(13)$ \\
\hline $\mathrm{C}(35)-\mathrm{Pd}(1)-\mathrm{P}(2)$ & $163.00(10)$ & $\mathrm{C}(6)-\mathrm{Fe}(1)-\mathrm{C}(3)$ & $148.08(12)$ \\
\hline $\mathrm{P}(1)-\mathrm{Pd}(1)-\mathrm{P}(2)$ & $100.73(4)$ & $\mathrm{C}(7)-\mathrm{Fe}(1)-\mathrm{C}(3)$ & $116.19(13)$ \\
\hline $\operatorname{Cent}(1)-\mathrm{Fe}(1)-\operatorname{cent}(1)$ & $149.70(10)$ & $\mathrm{C}(8)-\mathrm{Fe}(1)-\mathrm{C}(3)$ & $109.07(13)$ \\
\hline $\mathrm{C}(10)-\mathrm{Fe}(1)-\mathrm{C}(1)$ & $115.19(13)$ & $\mathrm{C}(10)-\mathrm{Fe}(1)-\mathrm{C}(4)$ & $130.84(13)$ \\
\hline $\mathrm{C}(10)-\mathrm{Fe}(1)-\mathrm{C}(2)$ & $147.63(13)$ & $\mathrm{C}(1)-\mathrm{Fe}(1)-\mathrm{C}(4)$ & $68.87(12)$ \\
\hline $\mathrm{C}(1)-\mathrm{Fe}(1)-\mathrm{C}(2)$ & $40.85(12)$ & $\mathrm{C}(2)-\mathrm{Fe}(1)-\mathrm{C}(4)$ & $68.80(13)$ \\
\hline $\mathrm{C}(10)-\mathrm{Fe}(1)-\mathrm{C}(9)$ & $40.86(13)$ & $\mathrm{C}(9)-\mathrm{Fe}(1)-\mathrm{C}(4)$ & $107.80(13)$ \\
\hline $\mathrm{C}(1)-\mathrm{Fe}(1)-\mathrm{C}(9)$ & $146.93(13)$ & $\mathrm{C}(5)-\mathrm{Fe}(1)-\mathrm{C}(4)$ & $39.95(12)$ \\
\hline $\mathrm{C}(2)-\mathrm{Fe}(1)-\mathrm{C}(9)$ & $170.87(14)$ & $\mathrm{C}(6)-\mathrm{Fe}(1)-\mathrm{C}(4)$ & $170.83(13)$ \\
\hline $\mathrm{C}(10)-\mathrm{Fe}(1)-\mathrm{C}(5)$ & $108.47(13)$ & $\mathrm{C}(7)-\mathrm{Fe}(1)-\mathrm{C}(4)$ & $146.79(13)$ \\
\hline $\mathrm{C}(1)-\mathrm{Fe}(1)-\mathrm{C}(5)$ & $41.52(11)$ & $\mathrm{C}(8)-\mathrm{Fe}(1)-\mathrm{C}(4)$ & $114.66(13)$ \\
\hline $\mathrm{C}(2)-\mathrm{Fe}(1)-\mathrm{C}(5)$ & $68.80(13)$ & $\mathrm{C}(3)-\mathrm{Fe}(1)-\mathrm{C}(4)$ & $40.51(12)$ \\
\hline $\mathrm{C}(9)-\mathrm{Fe}(1)-\mathrm{C}(5)$ & $114.46(13)$ & $\mathrm{C}(1)-\mathrm{P}(1)-\mathrm{C}(17)$ & $103.35(15)$ \\
\hline $\mathrm{C}(10)-\mathrm{Fe}(1)-\mathrm{C}(6)$ & $41.01(11)$ & $\mathrm{C}(1)-\mathrm{P}(1)-\mathrm{C}(11)$ & $103.20(15)$ \\
\hline $\mathrm{C}(1)-\mathrm{Fe}(1)-\mathrm{C}(6)$ & $109.34(12)$ & $\mathrm{C}(17)-\mathrm{P}(1)-\mathrm{C}(11)$ & $105.80(15)$ \\
\hline $\mathrm{C}(2)-\mathrm{Fe}(1)-\mathrm{C}(6)$ & $116.15(12)$ & $\mathrm{C}(1)-\mathrm{P}(1)-\mathrm{Pd}(1)$ & $116.21(10)$ \\
\hline $\mathrm{C}(9)-\mathrm{Fe}(1)-\mathrm{C}(6)$ & $68.49(13)$ & $C(17)-P(1)-P d(1)$ & $115.46(12)$ \\
\hline$C(5)-F e(1)-C(6)$ & $132.95(13)$ & $\mathrm{C}(11)-\mathrm{P}(1)-\mathrm{Pd}(1)$ & $111.54(11)$ \\
\hline $\mathrm{C}(10)-\mathrm{Fe}(1)-\mathrm{C}(7)$ & $68.68(13)$ & $C(6)-P(2)-C(23)$ & $101.42(14)$ \\
\hline $\mathrm{C}(1)-\mathrm{Fe}(1)-\mathrm{C}(7)$ & $132.99(12)$ & $C(6)-P(2)-C(29)$ & $104.18(15)$ \\
\hline $\mathrm{C}(2)-\mathrm{Fe}(1)-\mathrm{C}(7)$ & $109.85(13)$ & $\mathrm{C}(23)-\mathrm{P}(2)-\mathrm{C}(29)$ & $103.24(15)$ \\
\hline $\mathrm{C}(9)-\mathrm{Fe}(1)-\mathrm{C}(7)$ & 68.02(13) & $C(6)-P(2)-P d(1)$ & $120.50(11)$ \\
\hline $\mathrm{C}(5)-\mathrm{Fe}(1)-\mathrm{C}(7)$ & $172.86(12)$ & $C(23)-P(2)-P d(1)$ & $114.29(10)$ \\
\hline$C(6)-F e(1)-C(7)$ & $40.74(12)$ & $C(29)-P(2)-P d(1)$ & $111.30(11)$ \\
\hline$C(10)-F e(1)-C(8)$ & $68.51(14)$ & $C(2)-C(1)-C(5)$ & $107.2(3)$ \\
\hline $\mathrm{C}(1)-\mathrm{Fe}(1)-\mathrm{C}(8)$ & $172.00(13)$ & $C(2)-C(1)-P(1)$ & $125.6(2)$ \\
\hline $\mathrm{C}(2)-\mathrm{Fe}(1)-\mathrm{C}(8)$ & $132.49(14)$ & $C(5)-C(1)-P(1)$ & $127.2(2)$ \\
\hline $\mathrm{C}(9)-\mathrm{Fe}(1)-\mathrm{C}(8)$ & $40.28(13)$ & $\mathrm{C}(2)-\mathrm{C}(1)-\mathrm{Fe}(1)$ & $69.72(17)$ \\
\hline $\mathrm{C}(5)-\mathrm{Fe}(1)-\mathrm{C}(8)$ & $145.54(12)$ & $\mathrm{C}(5)-\mathrm{C}(1)-\mathrm{Fe}(1)$ & $69.80(17)$ \\
\hline $\mathrm{C}(6)-\mathrm{Fe}(1)-\mathrm{C}(8)$ & $68.37(13)$ & $\mathrm{P}(1)-\mathrm{C}(1)-\mathrm{Fe}(1)$ & $125.50(17)$ \\
\hline $\mathrm{C}(7)-\mathrm{Fe}(1)-\mathrm{C}(8)$ & $40.43(12)$ & $\mathrm{C}(1)-\mathrm{C}(2)-\mathrm{C}(3)$ & $108.0(3)$ \\
\hline$C(10)-F e(1)-C(3)$ & $170.09(12)$ & $C(1)-C(2)-F e(1)$ & $69.44(17)$ \\
\hline $\mathrm{C}(1)-\mathrm{Fe}(1)-\mathrm{C}(3)$ & $68.53(13)$ & $\mathrm{C}(3)-\mathrm{C}(2)-\mathrm{Fe}(1)$ & $70.63(17)$ \\
\hline $\mathrm{C}(2)-\mathrm{Fe}(1)-\mathrm{C}(3)$ & $40.84(12)$ & $C(4)-C(3)-C(2)$ & $108.2(3)$ \\
\hline
\end{tabular}




\begin{tabular}{|c|c|c|c|}
\hline $\mathrm{C}(4)-\mathrm{C}(3)-\mathrm{Fe}(1)$ & $69.76(18)$ & $\mathrm{C}(18)-\mathrm{C}(17)-\mathrm{P}(1)$ & $121.2(3)$ \\
\hline $\mathrm{C}(2)-\mathrm{C}(3)-\mathrm{Fe}(1)$ & $68.53(18)$ & $C(19)-C(18)-C(17)$ & $119.3(4)$ \\
\hline $\mathrm{C}(5)-\mathrm{C}(4)-\mathrm{C}(3)$ & $107.8(3)$ & $C(20)-C(19)-C(18)$ & $121.0(4)$ \\
\hline $\mathrm{C}(5)-\mathrm{C}(4)-\mathrm{Fe}(1)$ & $69.38(18)$ & $C(19)-C(20)-C(21)$ & $119.4(4)$ \\
\hline $\mathrm{C}(3)-\mathrm{C}(4)-\mathrm{Fe}(1)$ & $69.73(17)$ & $C(22)-C(21)-C(20)$ & $120.6(4)$ \\
\hline$C(4)-C(5)-C(1)$ & 108.7(3) & $C(21)-C(22)-C(17)$ & $120.3(4)$ \\
\hline $\mathrm{C}(4)-\mathrm{C}(5)-\mathrm{Fe}(1)$ & $70.67(18)$ & $C(24)-C(23)-C(28)$ & 118.3(3) \\
\hline $\mathrm{C}(1)-\mathrm{C}(5)-\mathrm{Fe}(1)$ & $68.68(17)$ & $\mathrm{C}(24)-\mathrm{C}(23)-\mathrm{P}(2)$ & $122.5(2)$ \\
\hline$C(10)-C(6)-C(7)$ & 107.3(3) & C(28)-C(23)-P(2) & 119.2(3) \\
\hline $\mathrm{C}(10)-\mathrm{C}(6)-\mathrm{P}(2)$ & $128.6(2)$ & $C(25)-C(24)-C(23)$ & $121.5(3)$ \\
\hline $\mathrm{C}(7)-\mathrm{C}(6)-\mathrm{P}(2)$ & $124.1(2)$ & $C(26)-C(25)-C(24)$ & $120.1(3)$ \\
\hline $\mathrm{C}(10)-\mathrm{C}(6)-\mathrm{Fe}(1)$ & $68.48(18)$ & $C(25)-C(26)-C(27)$ & 119.7(3) \\
\hline $\mathrm{C}(7)-\mathrm{C}(6)-\mathrm{Fe}(1)$ & $69.68(17)$ & $C(26)-C(27)-C(28)$ & $120.8(3)$ \\
\hline $\mathrm{P}(2)-\mathrm{C}(6)-\mathrm{Fe}(1)$ & $128.00(17)$ & $C(27)-C(28)-C(23)$ & 119.5(3) \\
\hline $\mathrm{C}(8)-\mathrm{C}(7)-\mathrm{C}(6)$ & $108.2(3)$ & $C(30)-C(29)-C(34)$ & $118.2(3)$ \\
\hline $\mathrm{C}(8)-\mathrm{C}(7)-\mathrm{Fe}(1)$ & $69.84(18)$ & C(30)-C(29)-P(2) & $118.5(3)$ \\
\hline $\mathrm{C}(6)-\mathrm{C}(7)-\mathrm{Fe}(1)$ & $69.58(17)$ & $\mathrm{C}(34)-\mathrm{C}(29)-\mathrm{P}(2)$ & 123.3(3) \\
\hline $\mathrm{C}(9)-\mathrm{C}(8)-\mathrm{C}(7)$ & 108.1(3) & $C(31)-C(30)-C(29)$ & $121.2(4)$ \\
\hline $\mathrm{C}(9)-\mathrm{C}(8)-\mathrm{Fe}(1)$ & $69.40(18)$ & $C(30)-C(31)-C(32)$ & $120.5(4)$ \\
\hline $\mathrm{C}(7)-\mathrm{C}(8)-\mathrm{Fe}(1)$ & $69.74(18)$ & $\mathrm{C}(33)-\mathrm{C}(32)-\mathrm{C}(31)$ & $118.9(4)$ \\
\hline$C(8)-C(9)-C(10)$ & $108.4(3)$ & $C(32)-C(33)-C(34)$ & $121.6(4)$ \\
\hline $\mathrm{C}(8)-\mathrm{C}(9)-\mathrm{Fe}(1)$ & 70.31(18) & $C(33)-C(34)-C(29)$ & 119.6(3) \\
\hline $\mathrm{C}(10)-\mathrm{C}(9)-\mathrm{Fe}(1)$ & $68.89(18)$ & $C(36)-C(35)-P d(1)$ & $71.35(19)$ \\
\hline $\mathrm{C}(9)-\mathrm{C}(10)-\mathrm{C}(6)$ & $108.0(3)$ & $C(35)-C(36)-C(37)$ & $119.2(4)$ \\
\hline $\mathrm{C}(9)-\mathrm{C}(10)-\mathrm{Fe}(1)$ & 70.24(19) & $C(35)-C(36)-P d(1)$ & $71.69(19)$ \\
\hline $\mathrm{C}(6)-\mathrm{C}(10)-\mathrm{Fe}(1)$ & 70.51(18) & $C(37)-C(36)-P d(1)$ & $71.31(18)$ \\
\hline$C(12)-C(11)-C(16)$ & $118.9(3)$ & $C(36)-C(37)-P d(1)$ & $71.21(19)$ \\
\hline C(12)-C(11)-P(1) & 118.6(3) & $\mathrm{Cl}(2)-\mathrm{C}(38)-\mathrm{Cl}(3)$ & $112.8(2)$ \\
\hline $\mathrm{C}(16)-\mathrm{C}(11)-\mathrm{P}(1)$ & $122.0(3)$ & & \\
\hline $\mathrm{C}(11)-\mathrm{C}(12)-\mathrm{C}(13)$ & $120.6(3)$ & & \\
\hline$C(14)-C(13)-C(12)$ & 119.3(4) & & \\
\hline $\mathrm{C}(15)-\mathrm{C}(14)-\mathrm{C}(13)$ & 120.6(3) & & \\
\hline $\mathrm{C}(14)-\mathrm{C}(15)-\mathrm{C}(16)$ & $120.0(3)$ & & \\
\hline$C(15)-C(16)-C(11)$ & $120.5(4)$ & & \\
\hline $\mathrm{C}(22)-\mathrm{C}(17)-\mathrm{C}(18)$ & 119.3(3) & & \\
\hline C(22)-C(17)-P(1) & 119.3(3) & & \\
\hline
\end{tabular}


Table S24. Anisotropic displacement parameters $\left(\AA^{2} x 0^{3}\right)$ for 12. The anisotropic displacement factor exponent takes the form: $-2 \pi^{2}\left[\mathrm{~h}^{2} \mathrm{a}^{* 2} \mathrm{U}^{11}+\ldots+2 \mathrm{hk} \mathrm{a} \mathrm{a}^{*} \mathrm{U}^{12}\right]$

\begin{tabular}{|c|c|c|c|c|c|c|}
\hline & $\mathrm{U}^{11}$ & $\mathrm{U}^{22}$ & $\mathrm{U}^{33}$ & $\mathrm{U}^{23}$ & $\mathrm{U}^{13}$ & $\mathrm{U}^{12}$ \\
\hline $\operatorname{Pd}(1)$ & $18(1)$ & $31(1)$ & $22(1)$ & $3(1)$ & $2(1)$ & $-3(1)$ \\
\hline $\mathrm{Fe}(1)$ & $17(1)$ & $26(1)$ & $20(1)$ & $3(1)$ & $2(1)$ & $0(1)$ \\
\hline $\mathrm{Cl}(1)$ & $24(1)$ & $38(1)$ & $45(1)$ & $1(1)$ & $1(1)$ & $3(1)$ \\
\hline $\mathrm{P}(1)$ & $20(1)$ & $27(1)$ & $22(1)$ & $4(1)$ & $1(1)$ & $-1(1)$ \\
\hline $\mathrm{P}(2)$ & $20(1)$ & $26(1)$ & $20(1)$ & $1(1)$ & $3(1)$ & $0(1)$ \\
\hline $\mathrm{C}(1)$ & $18(2)$ & $28(2)$ & $21(2)$ & $6(1)$ & $1(1)$ & $-1(1)$ \\
\hline $\mathrm{C}(2)$ & $25(2)$ & $32(2)$ & $19(2)$ & $1(1)$ & $5(1)$ & $1(1)$ \\
\hline $\mathrm{C}(3)$ & $23(2)$ & $27(2)$ & $23(2)$ & $-2(1)$ & $-2(1)$ & $-2(1)$ \\
\hline$C(4)$ & $19(2)$ & $34(2)$ & $20(2)$ & $5(1)$ & $-1(1)$ & $-4(1)$ \\
\hline $\mathrm{C}(5)$ & $18(2)$ & $31(2)$ & $24(2)$ & $4(1)$ & $0(1)$ & $3(1)$ \\
\hline$C(6)$ & $21(2)$ & $26(2)$ & $20(2)$ & $0(1)$ & $-1(1)$ & $-1(1)$ \\
\hline$C(7)$ & $21(2)$ & $30(2)$ & $24(2)$ & $5(1)$ & $-1(1)$ & $2(1)$ \\
\hline $\mathrm{C}(8)$ & $26(2)$ & $33(2)$ & $25(2)$ & $9(2)$ & $1(1)$ & $-6(1)$ \\
\hline $\mathrm{C}(9)$ & $25(2)$ & $43(2)$ & $22(2)$ & $3(2)$ & $9(1)$ & $-2(2)$ \\
\hline$C(10)$ & $21(2)$ & $32(2)$ & $24(2)$ & $-2(1)$ & $4(1)$ & $4(1)$ \\
\hline$C(11)$ & $20(2)$ & $35(2)$ & $24(2)$ & $4(2)$ & $2(1)$ & $-3(1)$ \\
\hline$C(12)$ & $39(2)$ & $36(2)$ & $32(2)$ & $10(2)$ & $11(2)$ & $1(2)$ \\
\hline$C(13)$ & $47(2)$ & $53(3)$ & $27(2)$ & $7(2)$ & $14(2)$ & $8(2)$ \\
\hline$C(14)$ & $34(2)$ & $66(3)$ & $23(2)$ & $14(2)$ & $8(2)$ & $-3(2)$ \\
\hline$C(15)$ & $39(2)$ & $47(2)$ & $32(2)$ & $18(2)$ & $4(2)$ & $-8(2)$ \\
\hline$C(16)$ & $37(2)$ & $36(2)$ & $29(2)$ & $9(2)$ & $2(2)$ & $-5(2)$ \\
\hline$C(17)$ & $25(2)$ & $25(2)$ & $34(2)$ & $6(2)$ & $2(2)$ & $-1(1)$ \\
\hline$C(18)$ & $30(2)$ & $34(2)$ & $39(2)$ & $13(2)$ & $2(2)$ & $1(2)$ \\
\hline$C(19)$ & $34(2)$ & $44(2)$ & $58(3)$ & $19(2)$ & $9(2)$ & $12(2)$ \\
\hline$C(20)$ & $59(3)$ & $50(3)$ & $59(3)$ & $4(2)$ & $15(2)$ & $25(2)$ \\
\hline $\mathrm{C}(21)$ & $73(3)$ & $50(3)$ & $58(3)$ & $-15(2)$ & $0(3)$ & $18(2)$ \\
\hline $\mathrm{C}(22)$ & $47(2)$ & $38(2)$ & $33(2)$ & $-2(2)$ & $-7(2)$ & $10(2)$ \\
\hline$C(23)$ & $15(2)$ & $27(2)$ & $30(2)$ & $2(2)$ & $1(1)$ & $-3(1)$ \\
\hline$C(24)$ & $38(2)$ & $34(2)$ & $27(2)$ & $-1(2)$ & $-4(2)$ & $7(2)$ \\
\hline$C(25)$ & $49(2)$ & $36(2)$ & $28(2)$ & $0(2)$ & $-14(2)$ & $-1(2)$ \\
\hline
\end{tabular}




\begin{tabular}{lcccccc}
$\mathrm{C}(26)$ & $26(2)$ & $30(2)$ & $53(3)$ & $6(2)$ & $-13(2)$ & $0(2)$ \\
$\mathrm{C}(27)$ & $21(2)$ & $31(2)$ & $54(3)$ & $5(2)$ & $10(2)$ & $-2(1)$ \\
$\mathrm{C}(28)$ & $26(2)$ & $30(2)$ & $33(2)$ & $2(2)$ & $8(2)$ & $-2(1)$ \\
$\mathrm{C}(29)$ & $29(2)$ & $30(2)$ & $18(2)$ & $0(1)$ & $-3(1)$ & $1(1)$ \\
$\mathrm{C}(30)$ & $33(2)$ & $40(2)$ & $36(2)$ & $-4(2)$ & $7(2)$ & $-4(2)$ \\
$\mathrm{C}(31)$ & $52(3)$ & $33(2)$ & $53(3)$ & $-13(2)$ & $1(2)$ & $-7(2)$ \\
$\mathrm{C}(32)$ & $64(3)$ & $35(2)$ & $36(2)$ & $-13(2)$ & $-2(2)$ & $5(2)$ \\
$\mathrm{C}(33)$ & $50(2)$ & $54(3)$ & $30(2)$ & $-8(2)$ & $11(2)$ & $12(2)$ \\
$\mathrm{C}(34)$ & $38(2)$ & $41(2)$ & $24(2)$ & $-4(2)$ & $4(2)$ & $0(2)$ \\
$\mathrm{C}(35)$ & $20(2)$ & $54(2)$ & $38(2)$ & $11(2)$ & $14(2)$ & $-9(2)$ \\
$\mathrm{C}(36)$ & $18(2)$ & $46(2)$ & $43(2)$ & $1(2)$ & $6(2)$ & $-12(2)$ \\
$\mathrm{C}(37)$ & $14(2)$ & $47(2)$ & $37(2)$ & $0(2)$ & $3(1)$ & $-3(1)$ \\
$\mathrm{C}(38)$ & $73(3)$ & $52(3)$ & $51(3)$ & $-16(2)$ & $-8(2)$ & $0(2)$ \\
$\mathrm{Cl}(2)$ & $122(1)$ & $59(1)$ & $73(1)$ & $-5(1)$ & $-26(1)$ & $13(1)$ \\
$\mathrm{Cl}(3)$ & $75(1)$ & $52(1)$ & $79(1)$ & $-13(1)$ & $-5(1)$ & $8(1)$ \\
& & & & & & \\
\hline
\end{tabular}


Table S25. Hydrogen coordinates $\left(\begin{array}{lll}x & 10^{4}\end{array}\right)$ and isotropic displacement parameters $\left(\AA^{2} x 1^{3}\right)$ for 12 .

\begin{tabular}{|c|c|c|c|c|}
\hline & $\mathrm{x}$ & $\mathrm{y}$ & $\mathrm{z}$ & $\mathrm{U}(\mathrm{eq})$ \\
\hline $\mathrm{H}(2 \mathrm{~A})$ & 5424 & 708 & 3642 & 30 \\
\hline $\mathrm{H}(3 \mathrm{~A})$ & 3150 & 56 & 3690 & 30 \\
\hline $\mathrm{H}(4 \mathrm{~A})$ & 1006 & 788 & 3719 & 30 \\
\hline $\mathrm{H}(5 \mathrm{~A})$ & 1937 & 1883 & 3670 & 29 \\
\hline $\mathrm{H}(7 \mathrm{~A})$ & 5470 & 429 & 5413 & 30 \\
\hline $\mathrm{H}(8 \mathrm{~A})$ & 2814 & 182 & 5453 & 34 \\
\hline $\mathrm{H}(9 \mathrm{~A})$ & 1437 & 1192 & 5430 & 35 \\
\hline $\mathrm{H}(10 \mathrm{~A})$ & 3224 & 2073 & 5367 & 30 \\
\hline $\mathrm{H}(12 \mathrm{~A})$ & 5760 & 1256 & 2573 & 42 \\
\hline $\mathrm{H}(13 \mathrm{~A})$ & 6452 & 1291 & 1430 & 50 \\
\hline $\mathrm{H}(14 \mathrm{~A})$ & 6641 & 2220 & 877 & 49 \\
\hline $\mathrm{H}(15 \mathrm{~A})$ & 6090 & 3107 & 1436 & 47 \\
\hline $\mathrm{H}(16 \mathrm{~A})$ & 5408 & 3075 & 2572 & 41 \\
\hline $\mathrm{H}(18 \mathrm{~A})$ & 2722 & 2697 & 2881 & 41 \\
\hline $\mathrm{H}(19 \mathrm{~A})$ & 1247 & 3490 & 3159 & 54 \\
\hline $\mathrm{H}(20 \mathrm{~A})$ & 1720 & 4025 & 4205 & 66 \\
\hline $\mathrm{H}(21 \mathrm{~A})$ & 3709 & 3783 & 4978 & 73 \\
\hline $\mathrm{H}(22 \mathrm{~A})$ & 5180 & 2990 & 4725 & 48 \\
\hline $\mathrm{H}(24 \mathrm{~A})$ & 7225 & 1497 & 6886 & 40 \\
\hline $\mathrm{H}(25 \mathrm{~A})$ & 8774 & 852 & 7536 & 47 \\
\hline $\mathrm{H}(26 \mathrm{~A})$ & 10219 & 209 & 6972 & 45 \\
\hline $\mathrm{H}(27 \mathrm{~A})$ & 10060 & 192 & 5747 & 42 \\
\hline $\mathrm{H}(28 \mathrm{~A})$ & 8511 & 843 & 5076 & 35 \\
\hline $\mathrm{H}(30 \mathrm{~A})$ & 7999 & 2879 & 5603 & 43 \\
\hline $\mathrm{H}(31 \mathrm{~A})$ & 7952 & 3705 & 6342 & 55 \\
\hline $\mathrm{H}(32 \mathrm{~A})$ & 6270 & 3765 & 7153 & 55 \\
\hline $\mathrm{H}(33 \mathrm{~A})$ & 4683 & 2976 & 7221 & 53 \\
\hline $\mathrm{H}(34 \mathrm{~A})$ & 4755 & 2121 & 6510 & 41 \\
\hline
\end{tabular}




$\begin{array}{lrrrr}\mathrm{H}(35 \mathrm{~A}) & 9104 & 2101 & 3281 & 44 \\ \mathrm{H}(35 \mathrm{~B}) & 8233 & 2751 & 3264 & 44 \\ \mathrm{H}(36 \mathrm{~A}) & 9376 & 2974 & 4373 & 43 \\ \mathrm{H}(37 \mathrm{~A}) & 9819 & 2199 & 5203 & 39 \\ \mathrm{H}(37 \mathrm{~B}) & 10143 & 1736 & 4557 & 39 \\ \mathrm{H}(38 \mathrm{~B}) & 5302 & -396 & 3120 & 72 \\ \mathrm{H}(38 \mathrm{~A}) & 6686 & -72 & 2891 & 72 \\ \end{array}$




\section{Experimental Procedure for the X-Ray Diffraction of 18}

\section{$\underline{\text { Data Collection }}$}

A red-orange plate crystal of $\mathrm{C}_{46} \mathrm{H}_{39} \mathrm{BrOP}_{2} \mathrm{Pd}$ having approximate dimensions of $0.15 \mathrm{x}$ $0.08 \times 0.08 \mathrm{~mm}^{3}$ was mounted with epoxy cement on the tip of a fine glass fiber. All measurements were made on a Nonius KappaCCD diffractometer with graphite monochromated Mo-K $\alpha$ radiation.

Cell constants and an orientation matrix for data collection corresponded to a primitive monoclinic cell with dimensions:

$$
\begin{array}{ll}
\mathrm{a}=17.295(4) \AA & \alpha=90^{\mathrm{o}} \\
\mathrm{b}=10.086(2) \AA & \beta=94.23(3)^{\circ} \\
\mathrm{c}=21.398(4) \AA & \gamma=90^{\circ} \\
\mathrm{V}=3722.4(13) \AA^{3} &
\end{array}
$$

For $Z=4$ and F.W. $=856.02$, the calculated density is $1.527 \mathrm{~g} / \mathrm{cm}^{3}$. Based on a statistical analysis of intensity distribution, and the successful solution and refinement of the structure, the space group was determined to be $P 2_{1} / c$ (\#14).

The data were collected at a temperature of $173(2) \mathrm{K}$ to a maximum $2 \theta$ value of $56.66^{\circ}$. Four omega scans consisting of 77, 84, 77, and 49 data frames, respectively, were collected with a frame width of $0.9^{\circ}$ and a detector-to-crystal distance, Dx, of $35.0 \mathrm{~mm}$. Each frame was exposed twice (for the purpose of de-zingering) for a total of $180 \mathrm{~s}$. The data frames were processed and scaled using the DENZO software package. ${ }^{1}$

\section{$\underline{\text { Data Reduction }}$}

A total of 16406 reflections were collected of which 9232 were unique and observed $\left(R_{\text {int }}\right.$ $=0.0806)$. The linear absorption coefficient, $\mu$, for Mo-K $\alpha$ radiation is $16.94 \mathrm{~cm}^{-1}$, and no absorption correction was applied. The data were corrected for Lorentz and polarization effects.

\section{$\underline{\text { Structure Solution and Refinement }}$}

The structure was solved by direct methods and expanded using Fourier techniques ${ }^{2}$. The non-hydrogen atoms were refined anisotropically, and hydrogen atoms were treated as idealized contributions. The final cycle of full-matrix least-squares refinement ${ }^{3}$ on $\mathrm{F}$ was based on 9232 observed reflections (I $>2.00 \sigma(\mathrm{I})$ ) and 472 variable parameters and converged with unweighted and weighted agreement factors of:

$$
\begin{gathered}
\mathrm{R}=\Sigma|| \mathrm{Fo}|-| \mathrm{Fc} \| / \Sigma|\mathrm{Fo}|=0.0525 \\
\mathrm{R}_{\mathrm{W}}=\left\{\Sigma\left[\mathrm{w}\left(\mathrm{F}_{\mathrm{o}}{ }^{2}-\mathrm{F}_{\mathrm{c}}{ }^{2}\right)^{2}\right] / \Sigma\left[\mathrm{w}\left(\mathrm{F}_{\mathrm{o}}{ }^{2}\right)^{2}\right]\right\}^{1 / 2}=0.0789
\end{gathered}
$$


The maximum and minimum peaks on the final difference Fourier map corresponded to 0.600 and $-1.022 \mathrm{e}^{-/} \AA^{3}$ respectively.

\section{REFERENCES}

(1) Z. Otwinowski and W. Minor, "Processing of X-Ray Diffraction Data Collected in Oscillation Mode," Methods in Enzymology, vol. 276: Macromolecular Crystallography, part A, 307-326, 1997, C.W. Carter, Jr. \& R.M. Sweet, Eds., Academic Press.

(2) Acta Cryst. A46 (1990) 467-473

(3) Least Squares function minimized:

$$
\Sigma w\left(\mathrm{~F}_{\mathrm{o}}^{2}-\mathrm{F}_{\mathrm{c}}{ }^{2}\right)^{2}
$$




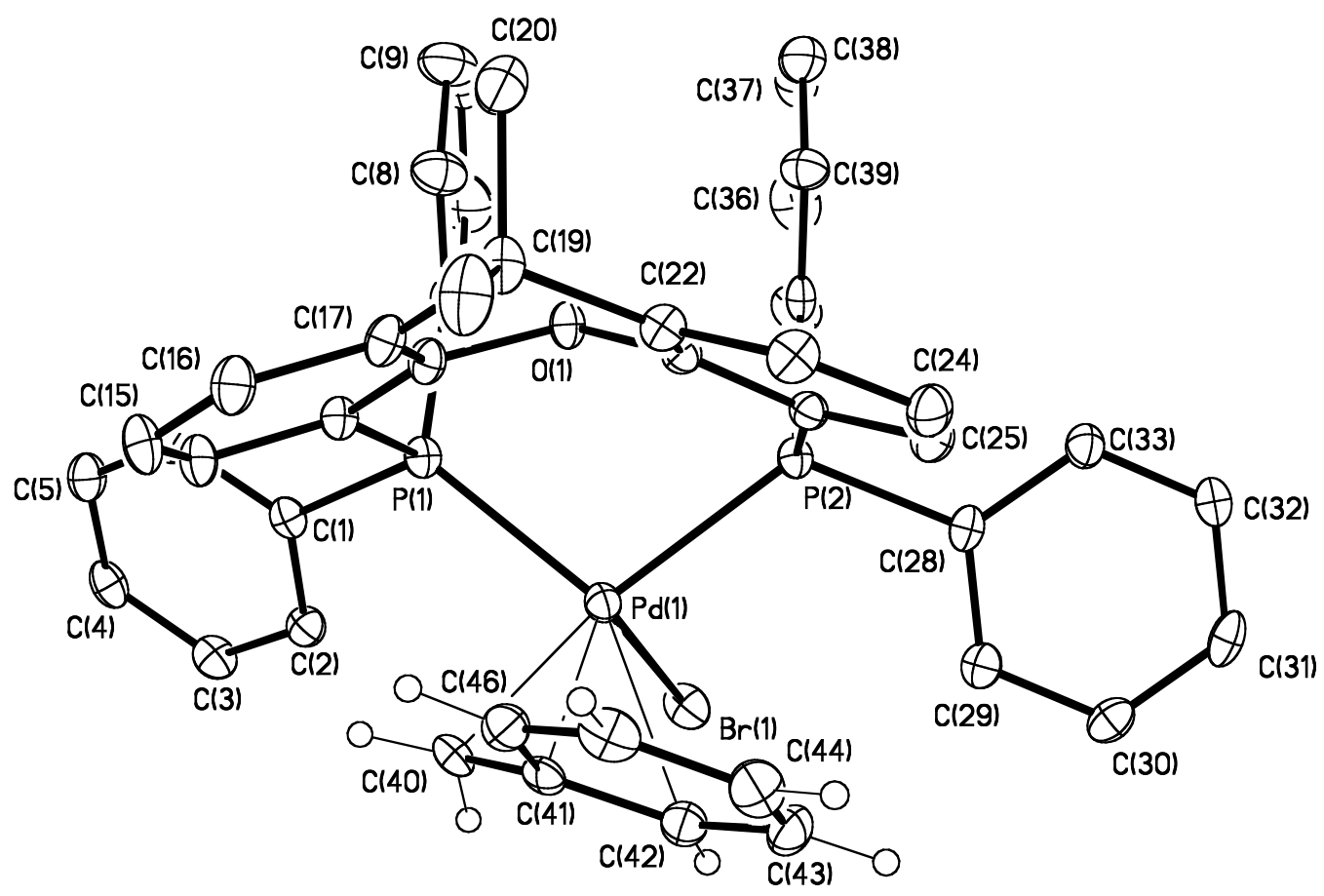

Figure S11. ORTEP diagram of 18 
Table S26. Crystal data and structure refinement for 18.

Empirical formula

Formula weight

Temperature

Wavelength

Crystal system

Space group

Unit cell dimensions

Volume

Z

Density (calculated)

Absorption coefficient

$\mathrm{F}(000)$

Crystal size

Theta range for data collection

Index ranges

Reflections collected

Independent reflections

Completeness to theta $=28.33^{\circ}$

Absorption correction

Max. and min. transmission

Refinement method

Data / restraints / parameters

Goodness-of-fit on $\mathrm{F}^{2}$

Final $\mathrm{R}$ indices [I $>2 \operatorname{sigma}(\mathrm{I})]$

$\mathrm{R}$ indices (all data)

Largest diff. peak and hole
$\mathrm{C}_{46} \mathrm{H}_{39} \mathrm{Br} \mathrm{O} \mathrm{P} 2 \mathrm{Pd}$

856.02

173(2) K

$0.71073 \AA$

Monoclinic

$\mathrm{P} 2(1) / \mathrm{c}$

$\mathrm{a}=17.295(4) \AA$

$\alpha=90^{\circ}$.

$\mathrm{b}=10.086(2) \AA$

$\beta=94.23(3)^{\circ}$.

$\mathrm{c}=21.398(4) \AA$

$\gamma=90^{\circ}$.

3722.4(13) $\AA^{3}$

4

$1.527 \mathrm{~g} / \mathrm{cm}^{3}$

$16.94 \mathrm{~cm}^{-1}$

1736

$0.15 \times 0.08 \times 0.08 \mathrm{~mm}^{3}$

2.17 to $28.33^{\circ}$.

$-22<=\mathrm{h}<=23,-12<=\mathrm{k}<=13,-28<=\mathrm{k}<=28$

16406

$9232[\mathrm{R}(\mathrm{int})=0.0806]$

$99.3 \%$

None

0.8764 and 0.7852

Full-matrix least-squares on $\mathrm{F}^{2}$

9232 / 0 / 472

1.001

$\mathrm{R} 1=0.0525, \mathrm{wR} 2=0.0789$

$\mathrm{R} 1=0.1239, \mathrm{wR} 2=0.0950$

0.600 and -1.022 e. $\AA^{-3}$ 
Table S27. Atomic coordinates ( $x 1^{4}$ ) and equivalent isotropic displacement parameters $\left(\AA^{2} \times 1^{3}\right)$ for 18 . U(eq) is defined as one third of the trace of the orthogonalized Uij tensor.

\begin{tabular}{|c|c|c|c|c|}
\hline & $\mathrm{x}$ & $\mathrm{y}$ & z & $\mathrm{U}(\mathrm{eq})$ \\
\hline $\operatorname{Pd}(1)$ & $2759(1)$ & $6350(1)$ & $845(1)$ & $25(1)$ \\
\hline $\operatorname{Br}(1)$ & $3025(1)$ & $4888(1)$ & $1915(1)$ & $35(1)$ \\
\hline $\mathrm{P}(1)$ & $3704(1)$ & $7878(1)$ & $685(1)$ & $24(1)$ \\
\hline $\mathrm{P}(2)$ & $1695(1)$ & $7619(1)$ & $1146(1)$ & $23(1)$ \\
\hline $\mathrm{O}(1)$ & $2330(1)$ & $9164(2)$ & 161(1) & $23(1)$ \\
\hline $\mathrm{C}(1)$ & $4708(2)$ & $7301(4)$ & $770(2)$ & $24(1)$ \\
\hline$C(2)$ & $4897(2)$ & $6093(4)$ & $1047(2)$ & $29(1)$ \\
\hline$C(3)$ & $5673(2)$ & $5738(4)$ & $1172(2)$ & $33(1)$ \\
\hline$C(4)$ & $6250(2)$ & $6588(4)$ & $1023(2)$ & $33(1)$ \\
\hline$C(5)$ & $6067(2)$ & $7796(4)$ & $749(2)$ & $34(1)$ \\
\hline$C(6)$ & $5304(2)$ & $8153(4)$ & $625(2)$ & $33(1)$ \\
\hline$C(7)$ & $3839(2)$ & $9355(4)$ & $1177(2)$ & $25(1)$ \\
\hline $\mathrm{C}(8)$ & $3660(2)$ & $10618(4)$ & $988(2)$ & $41(1)$ \\
\hline$C(9)$ & $3836(3)$ & $11683(5)$ & $1380(2)$ & $51(1)$ \\
\hline$C(10)$ & $4203(3)$ & $11506(5)$ & $1957(2)$ & $51(1)$ \\
\hline$C(11)$ & $4395(3)$ & $10239(5)$ & $2155(2)$ & $57(1)$ \\
\hline$C(12)$ & $4200(3)$ & $9171(5)$ & $1769(2)$ & $47(1)$ \\
\hline$C(13)$ & $3558(2)$ & $8461(4)$ & $-122(2)$ & $23(1)$ \\
\hline$C(14)$ & $4060(2)$ & $8227(4)$ & $-594(2)$ & $31(1)$ \\
\hline$C(15)$ & $3831(2)$ & $8531(4)$ & $-1215(2)$ & $34(1)$ \\
\hline$C(16)$ & $3107(2)$ & $9041(4)$ & $-1379(2)$ & $33(1)$ \\
\hline$C(17)$ & $2580(2)$ & $9281(4)$ & $-926(2)$ & $26(1)$ \\
\hline$C(18)$ & $2834(2)$ & $8980(4)$ & $-311(2)$ & $25(1)$ \\
\hline$C(19)$ & $1778(2)$ & 9881(4) & $-1055(2)$ & $29(1)$ \\
\hline$C(20)$ & $1814(2)$ & $11386(4)$ & $-890(2)$ & $40(1)$ \\
\hline$C(21)$ & $1470(2)$ & $9730(5)$ & $-1739(2)$ & $43(1)$ \\
\hline$C(22)$ & $1243(2)$ & $9209(4)$ & $-609(2)$ & $25(1)$ \\
\hline$C(23)$ & $468(2)$ & $8928(4)$ & $-748(2)$ & $29(1)$ \\
\hline$C(24)$ & $39(2)$ & $8333(4)$ & $-309(2)$ & $32(1)$ \\
\hline$C(25)$ & $369(2)$ & $7957(4)$ & $273(2)$ & $28(1)$ \\
\hline
\end{tabular}




\begin{tabular}{lrrrr}
$\mathrm{C}(26)$ & $1148(2)$ & $8219(3)$ & $435(2)$ & $22(1)$ \\
$\mathrm{C}(27)$ & $1558(2)$ & $8874(3)$ & $-12(2)$ & $23(1)$ \\
$\mathrm{C}(28)$ & $940(2)$ & $6770(4)$ & $1552(2)$ & $24(1)$ \\
$\mathrm{C}(29)$ & $946(2)$ & $5403(4)$ & $1601(2)$ & $28(1)$ \\
$\mathrm{C}(30)$ & $338(2)$ & $4749(4)$ & $1862(2)$ & $32(1)$ \\
$\mathrm{C}(31)$ & $-266(2)$ & $5458(4)$ & $2089(2)$ & $33(1)$ \\
$\mathrm{C}(32)$ & $-265(2)$ & $6814(4)$ & $2052(2)$ & $31(1)$ \\
$\mathrm{C}(33)$ & $331(2)$ & $7470(4)$ & $1790(2)$ & $29(1)$ \\
$\mathrm{C}(34)$ & $1880(2)$ & $9080(4)$ & $1630(2)$ & $25(1)$ \\
$\mathrm{C}(35)$ & $2279(2)$ & $8896(4)$ & $2215(2)$ & $39(1)$ \\
$\mathrm{C}(36)$ & $2464(3)$ & $9959(5)$ & $2598(2)$ & $49(1)$ \\
$\mathrm{C}(37)$ & $2275(3)$ & $11217(5)$ & $2412(2)$ & $45(1)$ \\
$\mathrm{C}(38)$ & $1877(3)$ & $11413(5)$ & $1840(2)$ & $46(1)$ \\
$\mathrm{C}(39)$ & $1676(2)$ & $10354(4)$ & $1447(2)$ & $33(1)$ \\
$\mathrm{C}(40)$ & $3360(2)$ & $5088(4)$ & $271(2)$ & $29(1)$ \\
$\mathrm{C}(41)$ & $2586(2)$ & $5030(4)$ & $-22(2)$ & $28(1)$ \\
$\mathrm{C}(42)$ & $1999(2)$ & $4430(4)$ & $300(2)$ & $33(1)$ \\
$\mathrm{C}(43)$ & $1225(3)$ & $4498(4)$ & $65(2)$ & $39(1)$ \\
$\mathrm{C}(44)$ & $1030(3)$ & $5136(4)$ & $-488(2)$ & $41(1)$ \\
$\mathrm{C}(45)$ & $1602(3)$ & $5752(4)$ & $-811(2)$ & $38(1)$ \\
$\mathrm{C}(46)$ & $2361(2)$ & $5721(4)$ & $-584(2)$ & $33(1)$ \\
& & & & \\
\hline
\end{tabular}


Table S28. Bond lengths $[\AA ̊]$ and angles $\left[^{\circ}\right]$ for 18 .

\begin{tabular}{|c|c|c|c|}
\hline $\operatorname{Pd}(1)-C(40)$ & $2.097(4)$ & $\mathrm{C}(19)-\mathrm{C}(22)$ & $1.534(5)$ \\
\hline $\mathrm{Pd}(1)-\mathrm{P}(1)$ & $2.2898(11)$ & $C(19)-C(20)$ & $1.558(5)$ \\
\hline $\operatorname{Pd}(1)-C(41)$ & $2.286(3)$ & $C(22)-C(27)$ & $1.393(5)$ \\
\hline $\mathrm{Pd}(1)-\mathrm{P}(2)$ & $2.3701(11)$ & $C(22)-C(23)$ & $1.381(5)$ \\
\hline $\operatorname{Pd}(1)-C(42)$ & $2.571(4)$ & $C(23)-C(24)$ & $1.378(5)$ \\
\hline $\operatorname{Pd}(1)-\operatorname{Br}(1)$ & $2.7345(7)$ & $C(24)-C(25)$ & $1.383(5)$ \\
\hline$P(1)-C(13)$ & $1.824(3)$ & $C(25)-C(26)$ & $1.391(5)$ \\
\hline $\mathrm{P}(1)-\mathrm{C}(1)$ & $1.828(4)$ & $C(26)-C(27)$ & $1.397(5)$ \\
\hline$P(1)-C(7)$ & $1.829(4)$ & $\mathrm{C}(28)-\mathrm{C}(29)$ & $1.383(5)$ \\
\hline$P(2)-C(34)$ & $1.816(4)$ & $\mathrm{C}(28)-\mathrm{C}(33)$ & $1.396(5)$ \\
\hline $\mathrm{P}(2)-\mathrm{C}(28)$ & $1.833(4)$ & $C(29)-C(30)$ & $1.391(5)$ \\
\hline$P(2)-C(26)$ & $1.835(4)$ & $\mathrm{C}(30)-\mathrm{C}(31)$ & $1.384(5)$ \\
\hline $\mathrm{O}(1)-\mathrm{C}(18)$ & $1.394(4)$ & $\mathrm{C}(31)-\mathrm{C}(32)$ & $1.370(5)$ \\
\hline $\mathrm{O}(1)-\mathrm{C}(27)$ & $1.392(4)$ & $C(32)-C(33)$ & $1.378(5)$ \\
\hline $\mathrm{C}(1)-\mathrm{C}(2)$ & $1.384(5)$ & $\mathrm{C}(34)-\mathrm{C}(39)$ & $1.381(5)$ \\
\hline$C(1)-C(6)$ & $1.395(5)$ & $C(34)-C(35)$ & $1.397(5)$ \\
\hline$C(2)-C(3)$ & $1.397(5)$ & $C(35)-C(36)$ & $1.372(6)$ \\
\hline$C(3)-C(4)$ & $1.370(5)$ & $C(36)-C(37)$ & $1.362(6)$ \\
\hline$C(4)-C(5)$ & $1.379(5)$ & $\mathrm{C}(37)-\mathrm{C}(38)$ & $1.374(6)$ \\
\hline$C(5)-C(6)$ & $1.374(5)$ & $\mathrm{C}(38)-\mathrm{C}(39)$ & $1.389(5)$ \\
\hline$C(7)-C(8)$ & $1.365(5)$ & $\mathrm{C}(40)-\mathrm{H}(40 \mathrm{~A})$ & $0.93(4)$ \\
\hline$C(7)-C(12)$ & $1.383(5)$ & $\mathrm{C}(40)-\mathrm{H}(40 \mathrm{~B})$ & $1.02(3)$ \\
\hline $\mathrm{C}(8)-\mathrm{C}(9)$ & $1.383(6)$ & $C(40)-C(41)$ & $1.437(5)$ \\
\hline C(9)-C(10) & $1.357(6)$ & $C(41)-C(42)$ & $1.406(5)$ \\
\hline$C(10)-C(11)$ & $1.380(6)$ & $C(41)-C(46)$ & $1.418(5)$ \\
\hline$C(11)-C(12)$ & $1.384(6)$ & $\mathrm{C}(42)-\mathrm{H}(42 \mathrm{~A})$ & $0.96(3)$ \\
\hline$C(13)-C(14)$ & $1.400(5)$ & $C(42)-C(43)$ & $1.397(6)$ \\
\hline$C(13)-C(18)$ & $1.389(5)$ & $C(43)-C(44)$ & $1.368(6)$ \\
\hline$C(14)-C(15)$ & $1.393(5)$ & $C(44)-C(45)$ & $1.393(6)$ \\
\hline$C(15)-C(16)$ & $1.375(5)$ & $C(45)-C(46)$ & $1.366(5)$ \\
\hline$C(16)-C(17)$ & $1.399(5)$ & & \\
\hline$C(17)-C(18)$ & $1.391(5)$ & $\mathrm{C}(40)-\mathrm{Pd}(1)-\mathrm{P}(1)$ & $86.06(13)$ \\
\hline$C(17)-C(19)$ & $1.520(5)$ & $C(40)-P d(1)-C(41)$ & $37.98(14)$ \\
\hline$C(19)-C(21)$ & $1.529(5)$ & $\mathrm{P}(1)-\mathrm{Pd}(1)-\mathrm{C}(41)$ & $108.87(10)$ \\
\hline
\end{tabular}




\begin{tabular}{|c|c|c|c|}
\hline $\mathrm{C}(40)-\mathrm{Pd}(1)-\mathrm{P}(2)$ & $156.26(12)$ & $\mathrm{C}(7)-\mathrm{C}(8)-\mathrm{C}(9)$ & $120.6(4)$ \\
\hline $\mathrm{P}(1)-\mathrm{Pd}(1)-\mathrm{P}(2)$ & $104.71(4)$ & $C(10)-C(9)-C(8)$ & $121.2(4)$ \\
\hline $\mathrm{C}(41)-\mathrm{Pd}(1)-\mathrm{P}(2)$ & $118.51(10)$ & $C(9)-C(10)-C(11)$ & $119.2(4)$ \\
\hline$C(40)-P d(1)-C(42)$ & $62.39(15)$ & $C(10)-C(11)-C(12)$ & $119.6(4)$ \\
\hline $\mathrm{P}(1)-\mathrm{Pd}(1)-\mathrm{C}(42)$ & $141.83(9)$ & $C(7)-C(12)-C(11)$ & $121.1(4)$ \\
\hline$C(41)-P d(1)-C(42)$ & $33.00(12)$ & $C(14)-C(13)-C(18)$ & $116.5(3)$ \\
\hline $\mathrm{P}(2)-\mathrm{Pd}(1)-\mathrm{C}(42)$ & $98.53(10)$ & $\mathrm{C}(14)-\mathrm{C}(13)-\mathrm{P}(1)$ & $125.4(3)$ \\
\hline $\mathrm{C}(40)-\mathrm{Pd}(1)-\mathrm{Br}(1)$ & $95.95(12)$ & $\mathrm{C}(18)-\mathrm{C}(13)-\mathrm{P}(1)$ & 117.1(3) \\
\hline $\mathrm{P}(1)-\mathrm{Pd}(1)-\mathrm{Br}(1)$ & $114.21(3)$ & $C(13)-C(14)-C(15)$ & $120.2(4)$ \\
\hline $\mathrm{C}(41)-\operatorname{Pd}(1)-\mathrm{Br}(1)$ & 111.71(10) & $C(16)-C(15)-C(14)$ & $121.0(3)$ \\
\hline $\mathrm{P}(2)-\mathrm{Pd}(1)-\mathrm{Br}(1)$ & $98.60(3)$ & $C(15)-C(16)-C(17)$ & $121.0(3)$ \\
\hline$C(42)-\operatorname{Pd}(1)-\operatorname{Br}(1)$ & $91.28(10)$ & $C(18)-C(17)-C(16)$ & $116.2(4)$ \\
\hline $\mathrm{C}(13)-\mathrm{P}(1)-\mathrm{C}(1)$ & $105.12(16)$ & $C(18)-C(17)-C(19)$ & $118.6(3)$ \\
\hline $\mathrm{C}(13)-\mathrm{P}(1)-\mathrm{C}(7)$ & $106.54(17)$ & $C(16)-C(17)-C(19)$ & $125.2(3)$ \\
\hline $\mathrm{C}(1)-\mathrm{P}(1)-\mathrm{C}(7)$ & $97.03(17)$ & $\mathrm{C}(17)-\mathrm{C}(18)-\mathrm{O}(1)$ & $119.1(3)$ \\
\hline$C(13)-P(1)-P d(1)$ & $107.91(12)$ & $C(17)-C(18)-C(13)$ & $124.9(3)$ \\
\hline $\mathrm{C}(1)-\mathrm{P}(1)-\mathrm{Pd}(1)$ & $117.00(13)$ & $\mathrm{O}(1)-\mathrm{C}(18)-\mathrm{C}(13)$ & $115.9(3)$ \\
\hline $\mathrm{C}(7)-\mathrm{P}(1)-\mathrm{Pd}(1)$ & $121.65(12)$ & $C(17)-C(19)-C(21)$ & $112.5(3)$ \\
\hline $\mathrm{C}(34)-\mathrm{P}(2)-\mathrm{C}(28)$ & $102.17(16)$ & $C(17)-C(19)-C(22)$ & $107.2(3)$ \\
\hline$C(34)-P(2)-C(26)$ & $105.27(17)$ & $C(21)-C(19)-C(22)$ & $111.6(3)$ \\
\hline$C(28)-P(2)-C(26)$ & $101.89(16)$ & $C(17)-C(19)-C(20)$ & $109.0(3)$ \\
\hline$C(34)-P(2)-P d(1)$ & $119.01(12)$ & $C(21)-C(19)-C(20)$ & $108.6(3)$ \\
\hline$C(28)-P(2)-P d(1)$ & $118.12(13)$ & $C(22)-C(19)-C(20)$ & $107.8(3)$ \\
\hline$C(26)-P(2)-P d(1)$ & $108.44(11)$ & $C(27)-C(22)-C(23)$ & $117.0(3)$ \\
\hline $\mathrm{C}(18)-\mathrm{O}(1)-\mathrm{C}(27)$ & $114.7(3)$ & $C(27)-C(22)-C(19)$ & $117.7(3)$ \\
\hline $\mathrm{C}(2)-\mathrm{C}(1)-\mathrm{C}(6)$ & $118.9(3)$ & $C(23)-C(22)-C(19)$ & $125.2(3)$ \\
\hline $\mathrm{C}(2)-\mathrm{C}(1)-\mathrm{P}(1)$ & $121.0(3)$ & $C(24)-C(23)-C(22)$ & $120.4(3)$ \\
\hline$C(6)-C(1)-P(1)$ & $119.7(3)$ & $C(23)-C(24)-C(25)$ & $121.7(4)$ \\
\hline$C(1)-C(2)-C(3)$ & $120.1(4)$ & $C(24)-C(25)-C(26)$ & $120.0(3)$ \\
\hline$C(4)-C(3)-C(2)$ & $120.0(4)$ & $C(27)-C(26)-C(25)$ & $116.7(3)$ \\
\hline$C(3)-C(4)-C(5)$ & $120.3(4)$ & $\mathrm{C}(27)-\mathrm{C}(26)-\mathrm{P}(2)$ & $117.8(3)$ \\
\hline$C(6)-C(5)-C(4)$ & $120.0(4)$ & $\mathrm{C}(25)-\mathrm{C}(26)-\mathrm{P}(2)$ & $125.1(3)$ \\
\hline$C(5)-C(6)-C(1)$ & $120.8(4)$ & $\mathrm{C}(22)-\mathrm{C}(27)-\mathrm{O}(1)$ & 119.7(3) \\
\hline$C(8)-C(7)-C(12)$ & $118.3(4)$ & $C(22)-C(27)-C(26)$ & $124.0(3)$ \\
\hline $\mathrm{C}(8)-\mathrm{C}(7)-\mathrm{P}(1)$ & $124.9(3)$ & $\mathrm{O}(1)-\mathrm{C}(27)-\mathrm{C}(26)$ & $116.3(3)$ \\
\hline $\mathrm{C}(12)-\mathrm{C}(7)-\mathrm{P}(1)$ & $116.6(3)$ & $C(29)-C(28)-C(33)$ & $118.6(3)$ \\
\hline
\end{tabular}




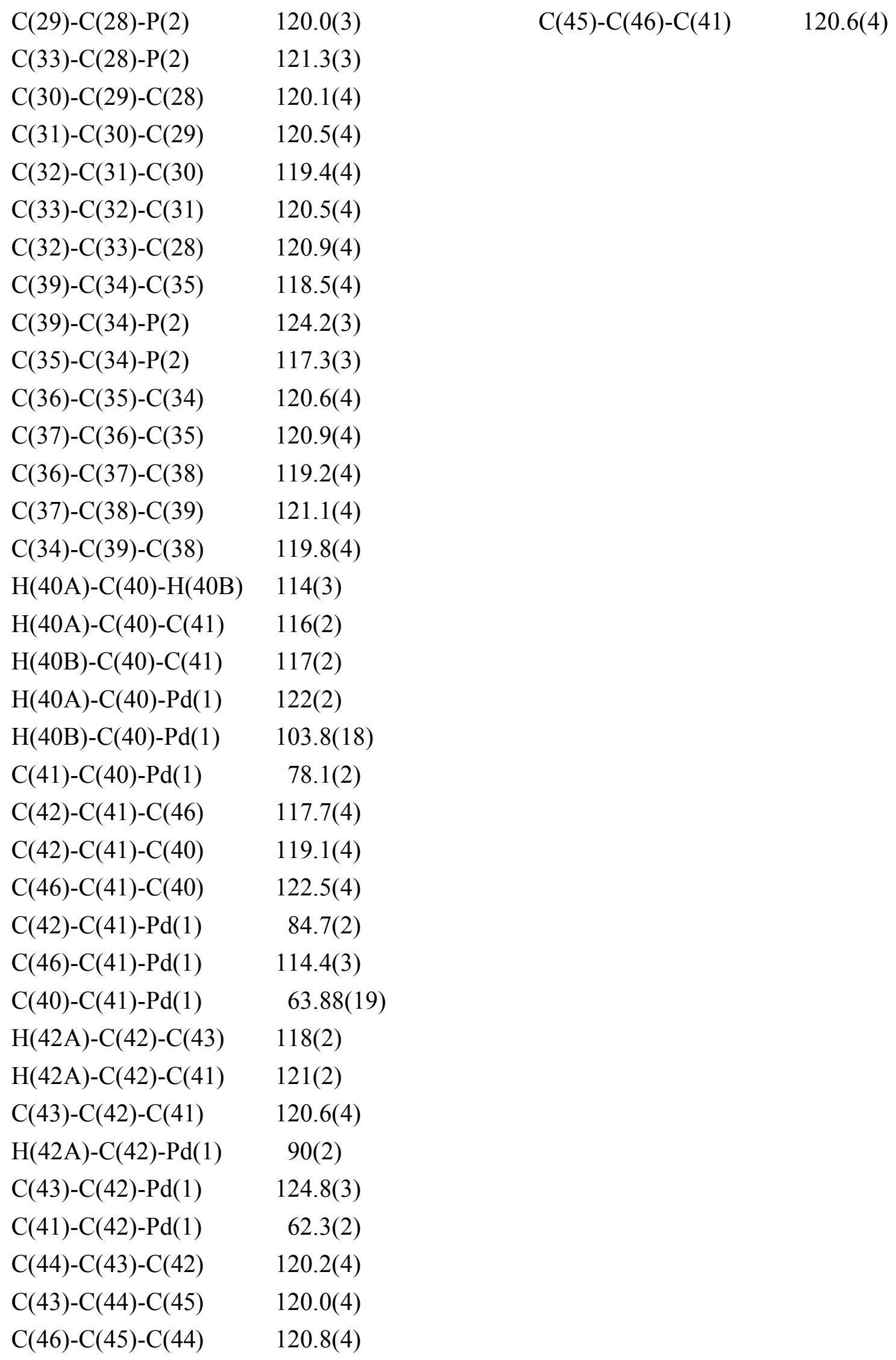


Table S29. Anisotropic displacement parameters $\left(\AA^{2} \times 10^{3}\right)$ for 18. The anisotropic displacement factor exponent takes the form: $-2 \pi^{2}\left[h^{2} a^{* 2} U^{11}+\ldots+2 h k a^{*} b^{*} U^{12}\right]$

\begin{tabular}{|c|c|c|c|c|c|c|}
\hline & $\mathrm{U}^{11}$ & $\mathrm{U}^{22}$ & $\mathrm{U}^{33}$ & $\mathrm{U}^{23}$ & $\mathrm{U}^{13}$ & $\mathrm{U}^{12}$ \\
\hline $\operatorname{Pd}(1)$ & $24(1)$ & $26(1)$ & $24(1)$ & $1(1)$ & $4(1)$ & $2(1)$ \\
\hline $\operatorname{Br}(1)$ & $44(1)$ & $39(1)$ & $23(1)$ & $5(1)$ & $3(1)$ & $10(1)$ \\
\hline $\mathrm{P}(1)$ & $20(1)$ & $29(1)$ & $22(1)$ & $1(1)$ & $-1(1)$ & $1(1)$ \\
\hline $\mathrm{P}(2)$ & $21(1)$ & $27(1)$ & $21(1)$ & $1(1)$ & $3(1)$ & $1(1)$ \\
\hline $\mathrm{O}(1)$ & $18(1)$ & $33(2)$ & $19(1)$ & $2(1)$ & $1(1)$ & $0(1)$ \\
\hline $\mathrm{C}(1)$ & $23(2)$ & $32(2)$ & $18(2)$ & $-4(2)$ & $-1(2)$ & $2(2)$ \\
\hline $\mathrm{C}(2)$ & $32(2)$ & $30(2)$ & $25(2)$ & $3(2)$ & $4(2)$ & $2(2)$ \\
\hline$C(3)$ & $35(3)$ & $36(2)$ & $28(2)$ & $5(2)$ & $-2(2)$ & $10(2)$ \\
\hline$C(4)$ & $23(2)$ & $47(3)$ & $28(2)$ & $-5(2)$ & $-1(2)$ & $9(2)$ \\
\hline$C(5)$ & $23(2)$ & $44(3)$ & $34(2)$ & $0(2)$ & $1(2)$ & $-1(2)$ \\
\hline$C(6)$ & $29(2)$ & $34(2)$ & $35(2)$ & $6(2)$ & $1(2)$ & $0(2)$ \\
\hline$C(7)$ & $16(2)$ & $31(2)$ & $26(2)$ & $-1(2)$ & $4(2)$ & $-2(2)$ \\
\hline $\mathrm{C}(8)$ & $40(3)$ & $36(3)$ & $47(3)$ & $-3(2)$ & $-8(2)$ & $6(2)$ \\
\hline $\mathrm{C}(9)$ & $47(3)$ & $35(3)$ & $70(3)$ & $-11(3)$ & $-5(3)$ & $3(2)$ \\
\hline$C(10)$ & $40(3)$ & $55(3)$ & $58(3)$ & $-31(3)$ & $15(3)$ & $-14(3)$ \\
\hline$C(11)$ & $66(4)$ & $72(4)$ & $31(3)$ & $-18(3)$ & $4(2)$ & $-4(3)$ \\
\hline$C(12)$ & $65(3)$ & $45(3)$ & $30(2)$ & $-2(2)$ & $3(2)$ & $-4(3)$ \\
\hline$C(13)$ & $20(2)$ & $28(2)$ & $21(2)$ & $2(2)$ & $0(2)$ & $1(2)$ \\
\hline$C(14)$ & $20(2)$ & $40(3)$ & $35(2)$ & $1(2)$ & $7(2)$ & $1(2)$ \\
\hline$C(15)$ & $30(2)$ & $50(3)$ & $23(2)$ & $1(2)$ & $9(2)$ & $5(2)$ \\
\hline$C(16)$ & $33(3)$ & $45(3)$ & $21(2)$ & $6(2)$ & $5(2)$ & $-2(2)$ \\
\hline$C(17)$ & $23(2)$ & $34(2)$ & $21(2)$ & $4(2)$ & $0(2)$ & $-6(2)$ \\
\hline$C(18)$ & $26(2)$ & $30(2)$ & $17(2)$ & $2(2)$ & $5(2)$ & $-3(2)$ \\
\hline$C(19)$ & $24(2)$ & $41(3)$ & $23(2)$ & $7(2)$ & $0(2)$ & $2(2)$ \\
\hline$C(20)$ & $28(2)$ & $43(3)$ & $47(3)$ & $17(2)$ & $0(2)$ & $3(2)$ \\
\hline$C(21)$ & $33(3)$ & $73(3)$ & $22(2)$ & $12(2)$ & $-2(2)$ & $-3(2)$ \\
\hline$C(22)$ & $26(2)$ & $28(2)$ & $20(2)$ & $2(2)$ & $-2(2)$ & $0(2)$ \\
\hline$C(23)$ & $29(2)$ & $34(2)$ & $24(2)$ & $3(2)$ & $-6(2)$ & $-1(2)$ \\
\hline$C(24)$ & $22(2)$ & $34(3)$ & $39(2)$ & $1(2)$ & $0(2)$ & $-3(2)$ \\
\hline$C(25)$ & $27(2)$ & $31(2)$ & $25(2)$ & $2(2)$ & $2(2)$ & $-2(2)$ \\
\hline
\end{tabular}




\begin{tabular}{lllllll}
$\mathrm{C}(26)$ & $23(2)$ & $21(2)$ & $21(2)$ & $1(2)$ & $3(2)$ & $2(2)$ \\
$\mathrm{C}(27)$ & $20(2)$ & $22(2)$ & $25(2)$ & $2(2)$ & $1(2)$ & $1(2)$ \\
$\mathrm{C}(28)$ & $19(2)$ & $34(2)$ & $18(2)$ & $3(2)$ & $-1(2)$ & $-4(2)$ \\
$\mathrm{C}(29)$ & $29(2)$ & $34(2)$ & $21(2)$ & $3(2)$ & $2(2)$ & $-2(2)$ \\
$\mathrm{C}(30)$ & $38(3)$ & $32(2)$ & $27(2)$ & $6(2)$ & $0(2)$ & $-8(2)$ \\
$\mathrm{C}(31)$ & $26(2)$ & $51(3)$ & $21(2)$ & $6(2)$ & $1(2)$ & $-14(2)$ \\
$\mathrm{C}(32)$ & $23(2)$ & $40(3)$ & $30(2)$ & $1(2)$ & $6(2)$ & $2(2)$ \\
$\mathrm{C}(33)$ & $27(2)$ & $32(2)$ & $30(2)$ & $3(2)$ & $6(2)$ & $0(2)$ \\
$\mathrm{C}(34)$ & $18(2)$ & $36(2)$ & $22(2)$ & $0(2)$ & $7(2)$ & $-2(2)$ \\
$\mathrm{C}(35)$ & $42(3)$ & $44(3)$ & $29(2)$ & $-4(2)$ & $-5(2)$ & $7(2)$ \\
$\mathrm{C}(36)$ & $44(3)$ & $72(4)$ & $31(2)$ & $-14(3)$ & $-4(2)$ & $0(3)$ \\
$\mathrm{C}(37)$ & $40(3)$ & $53(3)$ & $43(3)$ & $-24(3)$ & $11(2)$ & $-12(3)$ \\
$\mathrm{C}(38)$ & $53(3)$ & $37(3)$ & $51(3)$ & $-1(2)$ & $22(2)$ & $-3(2)$ \\
$\mathrm{C}(39)$ & $41(3)$ & $28(2)$ & $29(2)$ & $-1(2)$ & $3(2)$ & $1(2)$ \\
$\mathrm{C}(40)$ & $31(2)$ & $31(2)$ & $25(2)$ & $-6(2)$ & $6(2)$ & $7(2)$ \\
$\mathrm{C}(41)$ & $31(2)$ & $28(2)$ & $24(2)$ & $-13(2)$ & $5(2)$ & $-1(2)$ \\
$\mathrm{C}(42)$ & $34(3)$ & $34(3)$ & $30(2)$ & $-5(2)$ & $4(2)$ & $-2(2)$ \\
$\mathrm{C}(43)$ & $34(3)$ & $41(3)$ & $43(3)$ & $-9(2)$ & $8(2)$ & $-13(2)$ \\
$\mathrm{C}(44)$ & $32(3)$ & $51(3)$ & $40(3)$ & $-14(2)$ & $-6(2)$ & $-4(2)$ \\
$\mathrm{C}(45)$ & $45(3)$ & $41(3)$ & $25(2)$ & $-5(2)$ & $-8(2)$ & $5(2)$ \\
$\mathrm{C}(46)$ & $39(3)$ & $34(2)$ & $25(2)$ & $-5(2)$ & $6(2)$ & $-4(2)$ \\
& & & & & & \\
\hline
\end{tabular}


Table S30. Hydrogen coordinates $\left(\begin{array}{lll}x & 10^{4}\end{array}\right)$ and isotropic displacement parameters $\left(\AA^{2} x 1^{3}\right)$ for 18 .

\begin{tabular}{|c|c|c|c|c|}
\hline & $\mathrm{x}$ & $\mathrm{y}$ & z & $\mathrm{U}(\mathrm{eq})$ \\
\hline $\mathrm{H}(2 \mathrm{~A})$ & 4498 & 5503 & 1151 & 35 \\
\hline $\mathrm{H}(3 \mathrm{~A})$ & 5802 & 4906 & 1361 & 40 \\
\hline $\mathrm{H}(4 \mathrm{~A})$ & 6777 & 6345 & 1109 & 39 \\
\hline $\mathrm{H}(5 \mathrm{~A})$ & 6468 & 8382 & 645 & 40 \\
\hline $\mathrm{H}(6 \mathrm{~A})$ & 5182 & 8990 & 439 & 39 \\
\hline $\mathrm{H}(8 \mathrm{~A})$ & 3413 & 10767 & 582 & 50 \\
\hline $\mathrm{H}(9 \mathrm{~A})$ & 3697 & 12553 & 1243 & 61 \\
\hline $\mathrm{H}(10 \mathrm{~A})$ & 4325 & 12246 & 2220 & 61 \\
\hline $\mathrm{H}(11 \mathrm{~A})$ & 4660 & 10101 & 2555 & 68 \\
\hline $\mathrm{H}(12 \mathrm{~A})$ & 4317 & 8298 & 1913 & 56 \\
\hline $\mathrm{H}(14 \mathrm{~A})$ & 4559 & 7859 & -490 & 38 \\
\hline $\mathrm{H}(15 \mathrm{~A})$ & 4180 & 8384 & -1530 & 41 \\
\hline $\mathrm{H}(16 \mathrm{~A})$ & 2962 & 9233 & -1806 & 39 \\
\hline $\mathrm{H}(20 \mathrm{~A})$ & 2009 & 11496 & -451 & 59 \\
\hline $\mathrm{H}(20 \mathrm{~B})$ & 1294 & 11771 & -953 & 59 \\
\hline $\mathrm{H}(20 \mathrm{C})$ & 2163 & 11836 & -1162 & 59 \\
\hline $\mathrm{H}(21 \mathrm{~A})$ & 1446 & 8788 & -1850 & 64 \\
\hline $\mathrm{H}(21 \mathrm{~B})$ & 1816 & 10191 & -2010 & 64 \\
\hline $\mathrm{H}(21 \mathrm{C})$ & 949 & 10116 & -1797 & 64 \\
\hline $\mathrm{H}(23 \mathrm{~A})$ & 230 & 9146 & -1149 & 35 \\
\hline $\mathrm{H}(24 \mathrm{~A})$ & -498 & 8177 & -408 & 38 \\
\hline $\mathrm{H}(25 \mathrm{~A})$ & 64 & 7519 & 561 & 33 \\
\hline $\mathrm{H}(29 \mathrm{~A})$ & 1365 & 4909 & 1456 & 33 \\
\hline $\mathrm{H}(30 \mathrm{~A})$ & 339 & 3809 & 1885 & 39 \\
\hline $\mathrm{H}(31 \mathrm{~A})$ & -679 & 5008 & 2268 & 39 \\
\hline $\mathrm{H}(32 \mathrm{~A})$ & -678 & 7306 & 2209 & 37 \\
\hline$H(33 A)$ & 327 & 8411 & 1771 & 35 \\
\hline $\mathrm{H}(35 \mathrm{~A})$ & 2422 & 8027 & 2350 & 46 \\
\hline
\end{tabular}




\begin{tabular}{lllll}
$\mathrm{H}(36 \mathrm{~A})$ & 2728 & 9816 & 2997 & 59 \\
$\mathrm{H}(37 \mathrm{~A})$ & 2417 & 11950 & 2675 & 54 \\
$\mathrm{H}(38 \mathrm{~A})$ & 1737 & 12288 & 1712 & 55 \\
$\mathrm{H}(39 \mathrm{~A})$ & 1398 & 10504 & 1053 & 39 \\
$\mathrm{H}(40 \mathrm{~A})$ & $3740(20)$ & $5400(40)$ & $24(17)$ & $38(12)$ \\
$\mathrm{H}(40 \mathrm{~B})$ & $3530(20)$ & $4320(30)$ & $562(15)$ & $26(10)$ \\
$\mathrm{H}(42 \mathrm{~A})$ & $2120(20)$ & $3880(30)$ & $659(15)$ & $23(10)$ \\
$\mathrm{H}(43 \mathrm{~A})$ & 832 & 4099 & 290 & 47 \\
$\mathrm{H}(44 \mathrm{~A})$ & 505 & 5160 & -653 & 50 \\
$\mathrm{H}(45 \mathrm{~A})$ & 1463 & 6198 & -1194 & 45 \\
$\mathrm{H}(46 \mathrm{~A})$ & 2742 & 6168 & -804 & 39 \\
& & & & \\
\hline
\end{tabular}




\section{Experimental Procedure for the X-Ray Diffraction of 24}

\section{Data Collection}

A yellow plate crystal of $\mathrm{C}_{39} \mathrm{H}_{32} \mathrm{Cl}_{2} \mathrm{OP}_{2} \mathrm{Pd} \cdot 3.5 \mathrm{CH}_{2} \mathrm{Cl}_{2}, \mathrm{C}_{42.5} \mathrm{H}_{39} \mathrm{Cl}_{9} \mathrm{OP}_{2} \mathrm{Pd}$, having approximate dimensions of $0.20 \times 0.20 \times 0.20 \mathrm{~mm}$ was mounted with epoxy cement on the tip of a fine glass fiber. All measurements were made on a Nonius KappaCCD diffractometer with graphite monochromated Mo-Ka radiation.

Cell constants and an orientation matrix for data collection corresponded to a triclinic cell

with dimensions:

$$
\begin{array}{lll}
\mathrm{a}= & 10.981(2) \AA & \alpha=77.86(3) \mathrm{o} \\
\mathrm{b}= & 13.175(3) \AA & \beta=71.19(3) \mathrm{o} \\
\mathrm{c}= & 16.691(3) \AA & \gamma=85.61(3)^{\mathrm{o}} \\
\mathrm{V}= & 2234.6(8) \AA^{3} &
\end{array}
$$

For $\mathrm{Z}=2$ and F.W. $=1053.13$, the calculated density is $1.565 \mathrm{~g} / \mathrm{cm}^{3}$. Based on a statistical analysis of intensity distribution, and the successful solution and refinement of the structure, the space group was determined to be: $P-1(\# 2)$

The data were collected at a temperature of $183(2) \mathrm{K}$ to a maximum $2 \theta$ value of $52.00 \mathrm{o}$. Four omega scans consisting of 108, 97, 65, and 74 data frames, respectively, were collected with a frame width of $1.1^{\circ}$ and a detector-to-crystal distance, Dx, of $35 \mathrm{~mm}$. Each frame was exposed twice (for the purpose of de-zingering) for a total of 132 seconds. The data frames were processed and scaled using the DENZO software package. ${ }^{1}$

\section{Data Reduction}

A total of 13470 reflections were collected of which 8568 were unique and observed $\left(\mathrm{R}_{\text {int }}\right.$ $=0.0299$ ). The linear absorption coefficient, $\mu$, for Mo-K $\alpha$ radiation is $10.59 \mathrm{~cm}^{-1}$ and no absorption correction was applied. The data were corrected for Lorentz and polarization effects.

\section{Structure Solution and Refinement}

The structure was solved by direct methods and expanded using Fourier techniques ${ }^{2}$. The non-hydrogen atoms were refined anisotropically and hydrogen atoms were treated as idealized contributions. The final cycle of full-matrix least-squares refinement ${ }^{3}$ on $\mathrm{F}$ was based on 8568 
observed reflections (I $>2.00 \sigma(\mathrm{I}))$ and 498 variable parameters and converged with unweighted and weighted agreement factors of:

$$
\begin{gathered}
\mathrm{R}=\Sigma|| \mathrm{Fo}|-| \mathrm{Fc} \| / \Sigma|\mathrm{Fo}|=0.0644 \\
\mathrm{R}_{\mathrm{W}}=\left[\Sigma \mathrm{W}(|\mathrm{Fo}|-|\mathrm{Fc}|)^{2} / \Sigma \mathrm{W} \mathrm{Fo}^{2}\right]^{1 / 2}=0.1796
\end{gathered}
$$

The maximum and minimum peaks on the final difference Fourier map corresponded to 1.382 and $-1.051 \mathrm{e}^{-} / \AA^{3}$, respectively.

\section{REFERENCES}

(1) Z. Otwinowski and W. Minor, "Processing of X-Ray Diffraction Data Collected in Oscillation Mode," Methods in Enzymology, vol. 276: Macromolecular Crystallography, part A, 307-326, 1997, C.W. Carter, Jr. \& R.M. Sweet, Eds., Academic Press.

(2) Acta Cryst. A46 (1990) 467-473

(3) Least Squares function minimized:

$$
\Sigma w\left(\left|\mathrm{~F}_{\mathrm{O}}\right|-\left|\mathrm{F}_{\mathrm{c}}\right|\right)^{2}
$$




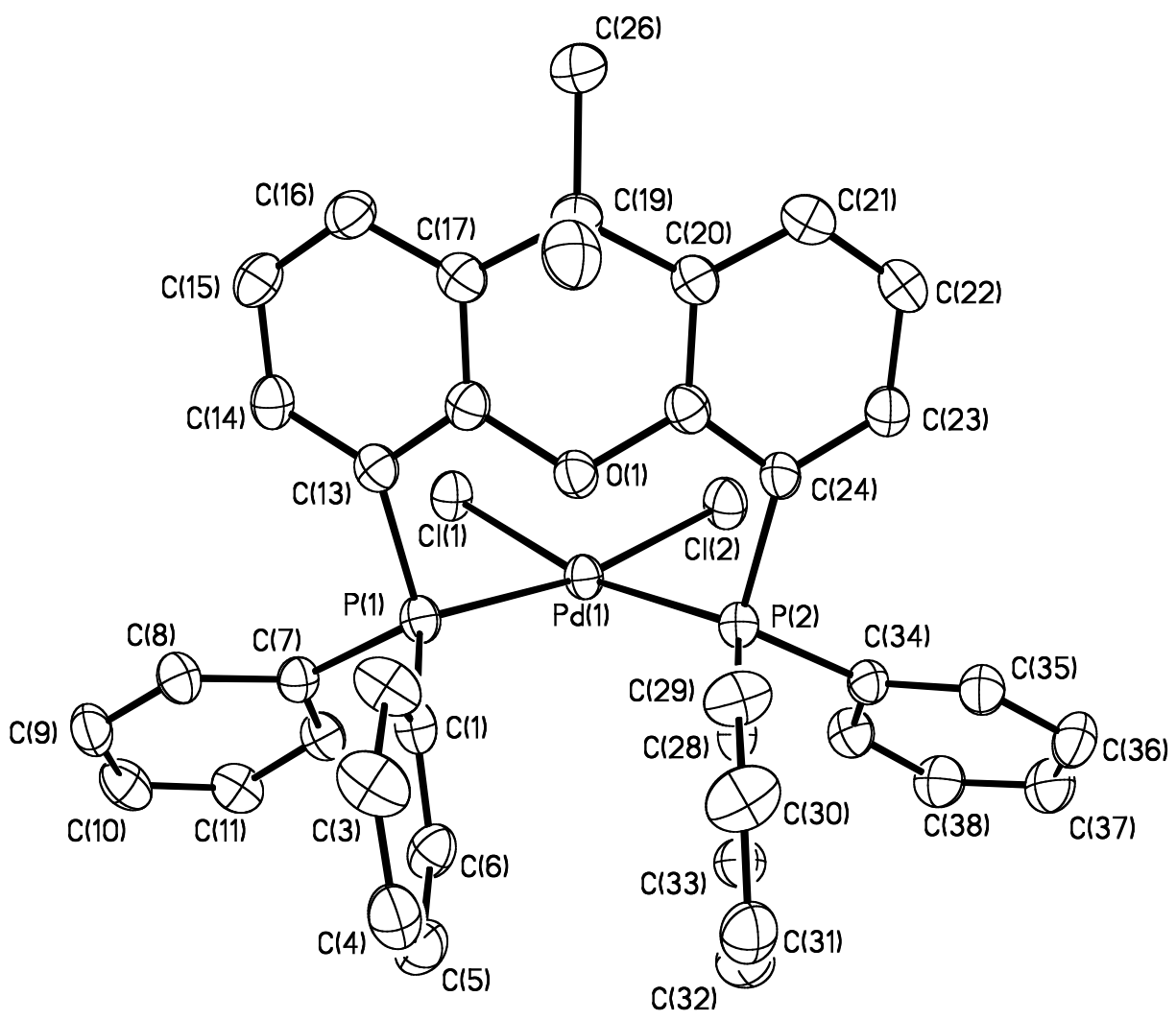

Figure S12. ORTEP diagram of 24 
Table S31. Crystal data and structure refinement for 24 .

Empirical formula

Formula weight

Temperature

Wavelength

Crystal system

Space group

Unit cell dimensions

Volume

Z

Density (calculated)

Absorption coefficient

$\mathrm{F}(000)$

Crystal size

Theta range for data collection

Index ranges

Reflections collected

Independent reflections

Completeness to theta $=26.00^{\circ}$

Absorption correction

Max. and min. transmission

Refinement method

Data / restraints / parameters

Goodness-of-fit on $\mathrm{F}^{2}$

Final $\mathrm{R}$ indices [I $>2 \operatorname{sigma}(\mathrm{I})]$

$\mathrm{R}$ indices (all data)

Extinction coefficient

Largest diff. peak and hole
$\mathrm{C}_{42.50} \mathrm{H}_{39} \mathrm{Cl}_{9} \mathrm{O} \mathrm{P}_{2} \mathrm{Pd}$

1053.13

183(2) K

$0.71073 \AA$

Triclinic

$\mathrm{P}-1$

$\mathrm{a}=10.981(2) \AA$

$\alpha=77.86(3)^{\circ}$.

$\mathrm{b}=13.175(3) \AA$

$\beta=71.19(3)^{\circ}$.

$\mathrm{c}=16.691(3) \AA$

$\gamma=85.61(3)^{\circ}$.

2234.6(8) $\AA^{3}$

2

$1.565 \mathrm{~g} / \mathrm{cm}^{3}$

$10.59 \mathrm{~cm}^{-1}$

1062

$0.20 \times 0.20 \times 0.20 \mathrm{~mm}^{3}$

3.23 to $26.00^{\circ}$.

$-12<=\mathrm{h}<=13,-16<=\mathrm{k}<=14,-20<=\mathrm{l}<=20$

13470

$8568[\mathrm{R}(\mathrm{int})=0.0299]$

$97.4 \%$

None

0.8162 and 0.8162

Full-matrix least-squares on $\mathrm{F}^{2}$

8568 / 6 / 498

1.276

$\mathrm{R} 1=0.0644, \mathrm{wR} 2=0.1796$

$\mathrm{R} 1=0.0866, \mathrm{wR} 2=0.1907$

$0.0105(19)$

1.382 and -1.051 e. $\AA^{-3}$ 
Table S32. Atomic coordinates ( $x \mathbf{1 0}^{4}$ ) and equivalent isotropic displacement parameters $\left(\AA^{2} \times 1^{3}\right)$ for 24 . U(eq) is defined as one third of the trace of the orthogonalized Uij tensor.

\begin{tabular}{|c|c|c|c|c|}
\hline & $\mathrm{x}$ & $\mathrm{y}$ & $\mathrm{z}$ & $\mathrm{U}(\mathrm{eq})$ \\
\hline $\operatorname{Pd}(1)$ & $1548(1)$ & $7233(1)$ & $3089(1)$ & $38(1)$ \\
\hline $\mathrm{P}(2)$ & $3251(1)$ & $7207(1)$ & $1869(1)$ & $40(1)$ \\
\hline $\mathrm{P}(1)$ & $2586(1)$ & $7482(1)$ & $4018(1)$ & $39(1)$ \\
\hline $\mathrm{Cl}(2)$ & $277(1)$ & $7263(1)$ & $2187(1)$ & $56(1)$ \\
\hline $\mathrm{Cl}(1)$ & $-315(1)$ & $7559(1)$ & $4181(1)$ & $49(1)$ \\
\hline $\mathrm{O}(1)$ & $3631(3)$ & $8973(3)$ & 2493(2) & $42(1)$ \\
\hline$C(1)$ & $4287(5)$ & $7186(4)$ & $3885(3)$ & $44(1)$ \\
\hline$C(2)$ & $5195(6)$ & $7970(5)$ & $3726(4)$ & $60(2)$ \\
\hline$C(3)$ & $6436(6)$ & $7682(6)$ & $3701(4)$ & $68(2)$ \\
\hline$C(4)$ & $6820(6)$ & $6678(5)$ & $3783(4)$ & $59(2)$ \\
\hline$C(5)$ & $5934(6)$ & $5917(5)$ & $3946(4)$ & $58(2)$ \\
\hline$C(6)$ & $4666(5)$ & $6172(4)$ & 3991(3) & $50(1)$ \\
\hline$C(7)$ & $1899(5)$ & $6818(4)$ & $5129(3)$ & $41(1)$ \\
\hline$C(8)$ & $2174(5)$ & $7129(4)$ & $5800(3)$ & $50(1)$ \\
\hline$C(9)$ & $1714(6)$ & $6576(5)$ & $6639(3)$ & $57(2)$ \\
\hline$C(10)$ & 1016(6) & $5677(5)$ & $6807(4)$ & $60(2)$ \\
\hline $\mathrm{C}(11)$ & $765(5)$ & $5340(5)$ & $6154(4)$ & $55(1)$ \\
\hline$C(12)$ & $1187(5)$ & $5914(4)$ & $5315(3)$ & $49(1)$ \\
\hline$C(13)$ & $2427(5)$ & $8889(4)$ & $3966(3)$ & $41(1)$ \\
\hline$C(14)$ & $1737(5)$ & $9402(4)$ & $4622(3)$ & $46(1)$ \\
\hline$C(15)$ & $1590(5)$ & $10462(4)$ & $4448(4)$ & $50(1)$ \\
\hline$C(16)$ & $2066(5)$ & $11039(4)$ & $3619(3)$ & $50(1)$ \\
\hline$C(17)$ & $2790(5)$ & $10563(4)$ & $2929(3)$ & $45(1)$ \\
\hline$C(18)$ & $2944(5)$ & 9494(4) & $3143(3)$ & $41(1)$ \\
\hline$C(19)$ & $3399(5)$ & 11091(4) & 2001(3) & $48(1)$ \\
\hline$C(20)$ & $3219(5)$ & $10372(4)$ & 1441(3) & $48(1)$ \\
\hline$C(21)$ & $2970(6)$ & $10674(5)$ & $663(4)$ & $62(2)$ \\
\hline$C(22)$ & $2831(7)$ & $9932(5)$ & $209(4)$ & $65(2)$ \\
\hline$C(23)$ & $2966(6)$ & $8870(4)$ & $527(3)$ & $52(1)$ \\
\hline$C(24)$ & $3254(5)$ & $8539(4)$ & 1294(3) & $41(1)$ \\
\hline
\end{tabular}




\begin{tabular}{|c|c|c|c|c|}
\hline$C(25)$ & $3380(5)$ & $9312(4)$ & $1718(3)$ & $43(1)$ \\
\hline$C(26)$ & $2832(7)$ & $12172(4)$ & $1790(4)$ & $63(2)$ \\
\hline$C(27)$ & $4864(6)$ & $11207(5)$ & $1842(4)$ & $64(2)$ \\
\hline $\mathrm{C}(28)$ & $4921(5)$ & $6899(4)$ & $1818(3)$ & $41(1)$ \\
\hline C(29) & $5864(6)$ & $7643(5)$ & $1573(4)$ & $62(2)$ \\
\hline$C(30)$ & $7115(6)$ & $7334(5)$ & $1513(5)$ & $69(2)$ \\
\hline $\mathrm{C}(31)$ & $7448(6)$ & $6316(5)$ & $1680(4)$ & $61(2)$ \\
\hline$C(32)$ & $6516(6)$ & $5561(5)$ & $1942(4)$ & $60(2)$ \\
\hline$C(33)$ & $5268(6)$ & $5859(4)$ & $2006(3)$ & $50(1)$ \\
\hline$C(34)$ & $3075(5)$ & $6372(4)$ & $1175(3)$ & $43(1)$ \\
\hline$C(35)$ & $3891(6)$ & $6476(5)$ & $334(3)$ & $52(1)$ \\
\hline$C(36)$ & $3788(7)$ & $5783(5)$ & $-170(4)$ & $63(2)$ \\
\hline$C(37)$ & $2966(7)$ & $4966(5)$ & $169(4)$ & $67(2)$ \\
\hline$C(38)$ & $2179(6)$ & $4824(5)$ & $1024(4)$ & $60(2)$ \\
\hline C(39) & $2252(6)$ & $5522(4)$ & $1518(4)$ & $51(1)$ \\
\hline$C(40)$ & $-6736(7)$ & $3298(7)$ & $4168(5)$ & $105(3)$ \\
\hline $\mathrm{Cl}(3)$ & $-5134(3)$ & $3208(2)$ & $3616(3)$ & $178(2)$ \\
\hline $\mathrm{Cl}(4)$ & $-7565(2)$ & $4027(2)$ & $3552(1)$ & $89(1)$ \\
\hline $\mathrm{C}(41)$ & $-645(7)$ & $9807(6)$ & $2325(5)$ & $88(2)$ \\
\hline $\mathrm{Cl}(5)$ & $-2005(3)$ & 9891(3) & 1991(2) & $138(1)$ \\
\hline $\mathrm{Cl}(6)$ & $-566(3)$ & $10805(2)$ & $2825(2)$ & $120(1)$ \\
\hline$C(42)$ & $-857(12)$ & $2692(8)$ & $267(10)$ & $166(6)$ \\
\hline $\mathrm{Cl}(7)$ & $-333(6)$ & $3574(4)$ & $683(4)$ & $248(3)$ \\
\hline $\mathrm{Cl}(8)$ & $-16(4)$ & $1577(4)$ & 153(3) & $156(2)$ \\
\hline $\mathrm{Cl}\left(8^{\prime}\right)$ & $-2316(11)$ & $2497(9)$ & $1299(7)$ & $119(3)$ \\
\hline
\end{tabular}


Table S33. Bond lengths $[\AA]]$ and angles $\left[^{\circ}\right]$ for 24 .

\begin{tabular}{|c|c|c|c|}
\hline $\mathrm{Pd}(1)-\mathrm{P}(2)$ & $2.2828(15)$ & $C(20)-C(21)$ & $1.384(8)$ \\
\hline $\operatorname{Pd}(1)-\mathrm{P}(1)$ & $2.2854(13)$ & $C(20)-C(25)$ & $1.391(7)$ \\
\hline $\operatorname{Pd}(1)-\mathrm{Cl}(1)$ & $2.3411(15)$ & $\mathrm{C}(21)-\mathrm{C}(22)$ & $1.401(9)$ \\
\hline $\mathrm{Pd}(1)-\mathrm{Cl}(2)$ & $2.3535(14)$ & $C(22)-C(23)$ & $1.403(8)$ \\
\hline$P(2)-C(24)$ & $1.814(5)$ & $C(23)-C(24)$ & $1.390(7)$ \\
\hline$P(2)-C(34)$ & $1.816(5)$ & $C(24)-C(25)$ & $1.395(7)$ \\
\hline $\mathrm{P}(2)-\mathrm{C}(28)$ & $1.826(5)$ & $\mathrm{C}(28)-\mathrm{C}(29)$ & $1.384(8)$ \\
\hline$P(1)-C(7)$ & $1.813(5)$ & $\mathrm{C}(28)-\mathrm{C}(33)$ & $1.393(7)$ \\
\hline $\mathrm{P}(1)-\mathrm{C}(1)$ & $1.829(5)$ & $C(29)-C(30)$ & $1.381(8)$ \\
\hline$P(1)-C(13)$ & $1.835(5)$ & $\mathrm{C}(30)-\mathrm{C}(31)$ & $1.358(9)$ \\
\hline $\mathrm{O}(1)-\mathrm{C}(25)$ & $1.385(6)$ & $C(31)-C(32)$ & $1.384(9)$ \\
\hline $\mathrm{O}(1)-\mathrm{C}(18)$ & $1.386(6)$ & $C(32)-C(33)$ & $1.373(8)$ \\
\hline$C(1)-C(6)$ & $1.360(8)$ & $C(34)-C(35)$ & $1.385(7)$ \\
\hline$C(1)-C(2)$ & $1.415(8)$ & $\mathrm{C}(34)-\mathrm{C}(39)$ & $1.397(8)$ \\
\hline$C(2)-C(3)$ & $1.376(8)$ & $C(35)-C(36)$ & $1.397(8)$ \\
\hline$C(3)-C(4)$ & $1.349(9)$ & $C(36)-C(37)$ & $1.362(9)$ \\
\hline$C(4)-C(5)$ & $1.375(9)$ & $\mathrm{C}(37)-\mathrm{C}(38)$ & $1.391(9)$ \\
\hline$C(5)-C(6)$ & $1.389(8)$ & $\mathrm{C}(38)-\mathrm{C}(39)$ & $1.381(8)$ \\
\hline$C(7)-C(8)$ & $1.395(7)$ & $\mathrm{C}(40)-\mathrm{Cl}(4)$ & $1.691(7)$ \\
\hline$C(7)-C(12)$ & $1.398(7)$ & $\mathrm{C}(40)-\mathrm{Cl}(3)$ & $1.711(7)$ \\
\hline $\mathrm{C}(8)-\mathrm{C}(9)$ & $1.385(8)$ & $\mathrm{C}(41)-\mathrm{Cl}(6)$ & $1.720(6)$ \\
\hline $\mathrm{C}(9)-\mathrm{C}(10)$ & $1.389(9)$ & $\mathrm{C}(41)-\mathrm{Cl}(5)$ & $1.743(7)$ \\
\hline$C(10)-C(11)$ & $1.369(9)$ & $\mathrm{C}(42)-\mathrm{Cl}(8)$ & $1.685(8)$ \\
\hline$C(11)-C(12)$ & $1.393(7)$ & $\mathrm{C}(42)-\mathrm{Cl}(7)$ & $1.693(8)$ \\
\hline$C(13)-C(14)$ & $1.388(7)$ & $\mathrm{C}(42)-\mathrm{Cl}\left(8^{\prime}\right)$ & $1.924(18)$ \\
\hline$C(13)-C(18)$ & $1.396(7)$ & & \\
\hline$C(14)-C(15)$ & $1.373(8)$ & $\mathrm{P}(2)-\mathrm{Pd}(1)-\mathrm{P}(1)$ & $100.61(5)$ \\
\hline$C(15)-C(16)$ & $1.385(8)$ & $\mathrm{P}(2)-\mathrm{Pd}(1)-\mathrm{Cl}(1)$ & $168.67(5)$ \\
\hline$C(16)-C(17)$ & $1.410(7)$ & $\mathrm{P}(1)-\mathrm{Pd}(1)-\mathrm{Cl}(1)$ & $84.30(5)$ \\
\hline$C(17)-C(18)$ & $1.391(7)$ & $\mathrm{P}(2)-\mathrm{Pd}(1)-\mathrm{Cl}(2)$ & $85.00(5)$ \\
\hline$C(17)-C(19)$ & $1.510(7)$ & $\mathrm{P}(1)-\mathrm{Pd}(1)-\mathrm{Cl}(2)$ & $169.40(5)$ \\
\hline$C(19)-C(20)$ & $1.527(7)$ & $\mathrm{Cl}(1)-\mathrm{Pd}(1)-\mathrm{Cl}(2)$ & $88.64(5)$ \\
\hline$C(19)-C(26)$ & $1.528(8)$ & $\mathrm{C}(24)-\mathrm{P}(2)-\mathrm{C}(34)$ & $107.5(2)$ \\
\hline$C(19)-C(27)$ & $1.559(9)$ & $\mathrm{C}(24)-\mathrm{P}(2)-\mathrm{C}(28)$ & $105.7(2)$ \\
\hline
\end{tabular}




\begin{tabular}{|c|c|c|c|}
\hline $\mathrm{C}(34)-\mathrm{P}(2)-\mathrm{C}(28)$ & $99.0(2)$ & $\mathrm{O}(1)-\mathrm{C}(18)-\mathrm{C}(17)$ & $118.3(4)$ \\
\hline$C(24)-P(2)-P d(1)$ & $101.53(16)$ & $\mathrm{O}(1)-\mathrm{C}(18)-\mathrm{C}(13)$ & $116.8(4)$ \\
\hline $\mathrm{C}(34)-\mathrm{P}(2)-\mathrm{Pd}(1)$ & $115.53(18)$ & $\mathrm{C}(17)-\mathrm{C}(18)-\mathrm{C}(13)$ & $124.9(4)$ \\
\hline $\mathrm{C}(28)-\mathrm{P}(2)-\mathrm{Pd}(1)$ & $126.35(15)$ & $C(17)-C(19)-C(20)$ & $106.8(4)$ \\
\hline $\mathrm{C}(7)-\mathrm{P}(1)-\mathrm{C}(1)$ & $99.1(2)$ & $C(17)-C(19)-C(26)$ & $112.4(4)$ \\
\hline$C(7)-P(1)-C(13)$ & 109.2(2) & $C(20)-C(19)-C(26)$ & $112.0(5)$ \\
\hline $\mathrm{C}(1)-\mathrm{P}(1)-\mathrm{C}(13)$ & $105.2(2)$ & $C(17)-C(19)-C(27)$ & $108.2(4)$ \\
\hline$C(7)-P(1)-P d(1)$ & 115.39(17) & $C(20)-C(19)-C(27)$ & $109.1(5)$ \\
\hline$C(1)-P(1)-P d(1)$ & $124.67(16)$ & $C(26)-C(19)-C(27)$ & $108.2(5)$ \\
\hline $\mathrm{C}(13)-\mathrm{P}(1)-\mathrm{Pd}(1)$ & $102.52(16)$ & $C(21)-C(20)-C(25)$ & $116.6(5)$ \\
\hline $\mathrm{C}(25)-\mathrm{O}(1)-\mathrm{C}(18)$ & $114.5(4)$ & $C(21)-C(20)-C(19)$ & $126.3(5)$ \\
\hline$C(6)-C(1)-C(2)$ & $119.4(5)$ & $C(25)-C(20)-C(19)$ & $117.0(4)$ \\
\hline $\mathrm{C}(6)-\mathrm{C}(1)-\mathrm{P}(1)$ & $118.3(4)$ & $C(20)-C(21)-C(22)$ & $120.6(5)$ \\
\hline $\mathrm{C}(2)-\mathrm{C}(1)-\mathrm{P}(1)$ & $122.2(4)$ & $\mathrm{C}(21)-\mathrm{C}(22)-\mathrm{C}(23)$ & $120.5(5)$ \\
\hline $\mathrm{C}(3)-\mathrm{C}(2)-\mathrm{C}(1)$ & $118.8(6)$ & $C(24)-C(23)-C(22)$ & $120.5(5)$ \\
\hline $\mathrm{C}(4)-\mathrm{C}(3)-\mathrm{C}(2)$ & $121.7(6)$ & $C(23)-C(24)-C(25)$ & $116.4(5)$ \\
\hline$C(3)-C(4)-C(5)$ & 119.3(6) & $\mathrm{C}(23)-\mathrm{C}(24)-\mathrm{P}(2)$ & $126.1(4)$ \\
\hline$C(4)-C(5)-C(6)$ & $120.7(6)$ & $\mathrm{C}(25)-\mathrm{C}(24)-\mathrm{P}(2)$ & $116.8(3)$ \\
\hline$C(1)-C(6)-C(5)$ & $119.9(5)$ & $\mathrm{O}(1)-\mathrm{C}(25)-\mathrm{C}(20)$ & $118.7(4)$ \\
\hline$C(8)-C(7)-C(12)$ & $118.6(5)$ & $\mathrm{O}(1)-\mathrm{C}(25)-\mathrm{C}(24)$ & $116.0(4)$ \\
\hline$C(8)-C(7)-P(1)$ & $121.1(4)$ & $C(20)-C(25)-C(24)$ & $125.2(5)$ \\
\hline $\mathrm{C}(12)-\mathrm{C}(7)-\mathrm{P}(1)$ & $119.9(4)$ & $\mathrm{C}(29)-\mathrm{C}(28)-\mathrm{C}(33)$ & $118.5(5)$ \\
\hline$C(9)-C(8)-C(7)$ & $120.8(5)$ & $\mathrm{C}(29)-\mathrm{C}(28)-\mathrm{P}(2)$ & $123.4(4)$ \\
\hline$C(8)-C(9)-C(10)$ & $119.4(5)$ & $\mathrm{C}(33)-\mathrm{C}(28)-\mathrm{P}(2)$ & $118.1(4)$ \\
\hline$C(11)-C(10)-C(9)$ & $120.8(5)$ & $C(30)-C(29)-C(28)$ & $119.3(6)$ \\
\hline$C(10)-C(11)-C(12)$ & $119.9(5)$ & $C(31)-C(30)-C(29)$ & $121.7(6)$ \\
\hline$C(11)-C(12)-C(7)$ & $120.4(5)$ & $C(30)-C(31)-C(32)$ & $119.9(6)$ \\
\hline$C(14)-C(13)-C(18)$ & $117.3(5)$ & $C(33)-C(32)-C(31)$ & $118.9(6)$ \\
\hline $\mathrm{C}(14)-\mathrm{C}(13)-\mathrm{P}(1)$ & $127.4(4)$ & $C(32)-C(33)-C(28)$ & $121.6(6)$ \\
\hline $\mathrm{C}(18)-\mathrm{C}(13)-\mathrm{P}(1)$ & $114.9(4)$ & $\mathrm{C}(35)-\mathrm{C}(34)-\mathrm{C}(39)$ & $118.8(5)$ \\
\hline$C(15)-C(14)-C(13)$ & $120.0(5)$ & $\mathrm{C}(35)-\mathrm{C}(34)-\mathrm{P}(2)$ & $120.3(4)$ \\
\hline$C(14)-C(15)-C(16)$ & $121.6(5)$ & $\mathrm{C}(39)-\mathrm{C}(34)-\mathrm{P}(2)$ & $120.2(4)$ \\
\hline$C(15)-C(16)-C(17)$ & $120.9(5)$ & $C(34)-C(35)-C(36)$ & $119.2(6)$ \\
\hline$C(18)-C(17)-C(16)$ & $115.2(5)$ & $C(37)-C(36)-C(35)$ & $121.2(6)$ \\
\hline $\mathrm{C}(18)-\mathrm{C}(17)-\mathrm{C}(19)$ & $117.8(4)$ & $\mathrm{C}(36)-\mathrm{C}(37)-\mathrm{C}(38)$ & $120.3(5)$ \\
\hline$C(16)-C(17)-C(19)$ & $127.0(5)$ & $C(39)-C(38)-C(37)$ & $118.8(6)$ \\
\hline
\end{tabular}




$\begin{array}{lr}\mathrm{C}(38)-\mathrm{C}(39)-\mathrm{C}(34) & 121.5(5) \\ \mathrm{Cl}(4)-\mathrm{C}(40)-\mathrm{Cl}(3) & 112.4(4) \\ \mathrm{Cl}(6)-\mathrm{C}(41)-\mathrm{Cl}(5) & 112.5(4) \\ \mathrm{Cl}(8)-\mathrm{C}(42)-\mathrm{Cl}(7) & 118.9(7) \\ \mathrm{Cl}(8)-\mathrm{C}(42)-\mathrm{Cl}\left(8^{\prime}\right) & 111.3(9) \\ \mathrm{Cl}(7)-\mathrm{C}(42)-\mathrm{Cl}\left(8^{\prime}\right) & 87.0(6)\end{array}$


Table S34. Anisotropic displacement parameters $\left(\AA^{2} \times 10^{3}\right)$ for 24. The anisotropic displacement factor exponent takes the form: $-2 \pi^{2}\left[\mathrm{~h}^{2} \mathrm{a}^{* 2} \mathrm{U}^{11}+\ldots+2 \mathrm{hk} \mathrm{a}^{*} \mathrm{~b}^{*} \mathrm{U}^{12}\right]$

\begin{tabular}{|c|c|c|c|c|c|c|}
\hline & $\mathrm{U}^{11}$ & $\mathrm{U}^{22}$ & $\mathrm{U}^{33}$ & $\mathrm{U}^{23}$ & $\mathrm{U}^{13}$ & $\mathrm{U}^{12}$ \\
\hline $\operatorname{Pd}(1)$ & $35(1)$ & $46(1)$ & $35(1)$ & $-8(1)$ & $-12(1)$ & $-4(1)$ \\
\hline $\mathrm{P}(2)$ & $41(1)$ & $43(1)$ & $34(1)$ & $-8(1)$ & $-10(1)$ & $-2(1)$ \\
\hline $\mathrm{P}(1)$ & $38(1)$ & $45(1)$ & $35(1)$ & $-7(1)$ & $-12(1)$ & $-7(1)$ \\
\hline $\mathrm{Cl}(2)$ & $48(1)$ & $74(1)$ & $52(1)$ & $-17(1)$ & $-23(1)$ & $1(1)$ \\
\hline $\mathrm{Cl}(1)$ & $37(1)$ & $65(1)$ & $44(1)$ & $-16(1)$ & $-10(1)$ & $-2(1)$ \\
\hline $\mathrm{O}(1)$ & $42(2)$ & $47(2)$ & $34(2)$ & $-6(1)$ & $-10(1)$ & $1(2)$ \\
\hline$C(1)$ & $41(3)$ & $56(3)$ & $33(2)$ & $-7(2)$ & $-11(2)$ & $-8(2)$ \\
\hline$C(2)$ & $47(3)$ & $60(4)$ & $69(4)$ & $9(3)$ & $-24(3)$ & $-14(3)$ \\
\hline$C(3)$ & $49(4)$ & $72(4)$ & $82(4)$ & $4(3)$ & $-29(3)$ & $-15(3)$ \\
\hline$C(4)$ & $47(3)$ & $83(4)$ & $46(3)$ & $-13(3)$ & $-15(3)$ & $-1(3)$ \\
\hline$C(5)$ & $52(3)$ & $69(4)$ & $63(3)$ & $-31(3)$ & $-21(3)$ & $12(3)$ \\
\hline$C(6)$ & $45(3)$ & $56(3)$ & $56(3)$ & $-23(3)$ & $-17(2)$ & $-3(2)$ \\
\hline$C(7)$ & $36(3)$ & $47(3)$ & $38(2)$ & $-9(2)$ & $-9(2)$ & $-2(2)$ \\
\hline$C(8)$ & $51(3)$ & $57(3)$ & $47(3)$ & $-10(2)$ & $-22(2)$ & $-4(3)$ \\
\hline$C(9)$ & $59(4)$ & $75(4)$ & $37(3)$ & $-11(3)$ & $-18(3)$ & $7(3)$ \\
\hline$C(10)$ & $52(3)$ & $68(4)$ & $47(3)$ & $10(3)$ & $-11(3)$ & $-6(3)$ \\
\hline$C(11)$ & $43(3)$ & $58(3)$ & $55(3)$ & $3(3)$ & $-10(3)$ & $-11(3)$ \\
\hline$C(12)$ & $43(3)$ & $57(3)$ & $47(3)$ & $-6(2)$ & $-17(2)$ & $-7(2)$ \\
\hline$C(13)$ & $38(3)$ & $46(3)$ & $44(3)$ & $-9(2)$ & $-19(2)$ & $-5(2)$ \\
\hline$C(14)$ & $36(3)$ & $63(3)$ & $38(3)$ & $-14(2)$ & $-7(2)$ & $-3(2)$ \\
\hline$C(15)$ & $46(3)$ & $50(3)$ & $55(3)$ & $-21(3)$ & $-10(2)$ & $-8(2)$ \\
\hline$C(16)$ & $55(3)$ & $43(3)$ & $56(3)$ & $-16(2)$ & $-20(3)$ & $1(2)$ \\
\hline$C(17)$ & $44(3)$ & $45(3)$ & $47(3)$ & $-5(2)$ & $-19(2)$ & $-6(2)$ \\
\hline $\mathrm{C}(18)$ & $38(3)$ & $46(3)$ & $43(3)$ & $-13(2)$ & $-15(2)$ & $-2(2)$ \\
\hline$C(19)$ & $57(3)$ & $40(3)$ & $47(3)$ & $-7(2)$ & $-15(2)$ & $-3(2)$ \\
\hline$C(20)$ & $53(3)$ & $46(3)$ & $40(3)$ & $-4(2)$ & $-11(2)$ & $-1(2)$ \\
\hline $\mathrm{C}(21)$ & $80(4)$ & $48(3)$ & $53(3)$ & $-2(3)$ & $-20(3)$ & $2(3)$ \\
\hline$C(22)$ & $87(5)$ & $61(4)$ & $44(3)$ & $-2(3)$ & $-24(3)$ & $8(3)$ \\
\hline$C(23)$ & $66(4)$ & $49(3)$ & $41(3)$ & $-11(2)$ & $-17(3)$ & $0(3)$ \\
\hline $\mathrm{C}(24)$ & $43(3)$ & $47(3)$ & $31(2)$ & $-7(2)$ & $-7(2)$ & $2(2)$ \\
\hline
\end{tabular}




\begin{tabular}{|c|c|c|c|c|c|c|}
\hline$C(25)$ & $40(3)$ & $49(3)$ & $37(2)$ & $-5(2)$ & $-10(2)$ & $-2(2)$ \\
\hline$C(26)$ & $85(5)$ & $43(3)$ & $57(3)$ & $-10(3)$ & $-18(3)$ & $4(3)$ \\
\hline$C(27)$ & $66(4)$ & $68(4)$ & $52(3)$ & $-5(3)$ & $-11(3)$ & $-21(3)$ \\
\hline $\mathrm{C}(28)$ & $43(3)$ & $51(3)$ & $29(2)$ & $-10(2)$ & $-7(2)$ & $-5(2)$ \\
\hline C(29) & $50(3)$ & $50(3)$ & $79(4)$ & $-13(3)$ & $-11(3)$ & $-2(3)$ \\
\hline$C(30)$ & $38(3)$ & $71(4)$ & $92(5)$ & $-22(4)$ & $-8(3)$ & $-7(3)$ \\
\hline $\mathrm{C}(31)$ & $46(3)$ & $80(4)$ & $57(3)$ & $-21(3)$ & $-15(3)$ & $6(3)$ \\
\hline$C(32)$ & $51(4)$ & $65(4)$ & $61(3)$ & $-11(3)$ & $-17(3)$ & $11(3)$ \\
\hline$C(33)$ & $50(3)$ & $49(3)$ & $52(3)$ & $-10(2)$ & $-17(2)$ & $2(2)$ \\
\hline$C(34)$ & $46(3)$ & $48(3)$ & $40(3)$ & $-13(2)$ & $-18(2)$ & $4(2)$ \\
\hline$C(35)$ & $53(3)$ & $57(3)$ & $44(3)$ & $-11(2)$ & $-13(2)$ & $2(3)$ \\
\hline$C(36)$ & $73(4)$ & $70(4)$ & $51(3)$ & $-28(3)$ & $-18(3)$ & $7(3)$ \\
\hline$C(37)$ & $82(5)$ & $72(4)$ & $69(4)$ & $-33(3)$ & $-41(4)$ & $6(4)$ \\
\hline$C(38)$ & $64(4)$ & $58(4)$ & $68(4)$ & $-19(3)$ & $-28(3)$ & $-6(3)$ \\
\hline C(39) & $53(3)$ & $53(3)$ & $52(3)$ & $-12(2)$ & $-20(3)$ & $-8(3)$ \\
\hline$C(40)$ & $117(7)$ & $116(7)$ & $82(5)$ & $3(5)$ & $-35(5)$ & $-49(6)$ \\
\hline $\mathrm{Cl}(3)$ & $89(2)$ & $104(2)$ & $315(5)$ & $51(2)$ & $-84(2)$ & $-7(2)$ \\
\hline $\mathrm{Cl}(4)$ & $75(1)$ & $90(1)$ & $97(1)$ & $-18(1)$ & $-22(1)$ & $-4(1)$ \\
\hline$C(41)$ & $92(6)$ & $84(5)$ & $94(5)$ & $-18(4)$ & $-39(5)$ & $-2(4)$ \\
\hline $\mathrm{Cl}(5)$ & 161(3) & $149(2)$ & $121(2)$ & $11(2)$ & $-81(2)$ & $-52(2)$ \\
\hline $\mathrm{Cl}(6)$ & $117(2)$ & $140(2)$ & $127(2)$ & $-54(2)$ & $-49(2)$ & $-12(2)$ \\
\hline$C(42)$ & $120(9)$ & $236(16)$ & 189(13) & $-92(12)$ & $-89(9)$ & $28(10)$ \\
\hline $\mathrm{Cl}(7)$ & $313(7)$ & $245(5)$ & $271(6)$ & $-5(4)$ & $-213(6)$ & $-95(5)$ \\
\hline $\mathrm{Cl}(8)$ & $110(3)$ & $190(4)$ & $121(3)$ & $46(3)$ & $-22(2)$ & $-5(3)$ \\
\hline $\mathrm{Cl}\left(8^{\prime}\right)$ & $109(7)$ & $145(8)$ & $106(6)$ & $17(6)$ & $-59(6)$ & $-20(6)$ \\
\hline
\end{tabular}


Table S35. Hydrogen coordinates ( $x \mathbf{1 0}^{4}$ ) and isotropic displacement parameters $\left(\AA^{2} \times 10^{3}\right)$ for 24.

\begin{tabular}{|c|c|c|c|c|}
\hline & $\mathrm{X}$ & $\mathrm{y}$ & $\mathrm{Z}$ & $\mathrm{U}(\mathrm{eq})$ \\
\hline $\mathrm{H}(2 \mathrm{~A})$ & 4952 & 8682 & 3638 & 72 \\
\hline $\mathrm{H}(3 \mathrm{~A})$ & 7038 & 8203 & 3623 & 81 \\
\hline $\mathrm{H}(4 \mathrm{~A})$ & 7694 & 6500 & 3728 & 70 \\
\hline $\mathrm{H}(5 \mathrm{~A})$ & 6193 & 5208 & 4028 & 70 \\
\hline $\mathrm{H}(6 \mathrm{~A})$ & 4065 & 5641 & 4096 & 60 \\
\hline $\mathrm{H}(8 \mathrm{~A})$ & 2683 & 7727 & 5680 & 60 \\
\hline $\mathrm{H}(9 \mathrm{~A})$ & 1876 & 6809 & 7097 & 68 \\
\hline $\mathrm{H}(10 \mathrm{~A})$ & 709 & 5292 & 7381 & 72 \\
\hline $\mathrm{H}(11 \mathrm{~A})$ & 305 & 4715 & 6274 & 66 \\
\hline $\mathrm{H}(12 \mathrm{~A})$ & 989 & 5691 & 4866 & 58 \\
\hline $\mathrm{H}(14 \mathrm{~A})$ & 1367 & 9020 & 5191 & 55 \\
\hline $\mathrm{H}(15 \mathrm{~A})$ & 1151 & 10808 & 4908 & 60 \\
\hline $\mathrm{H}(16 \mathrm{~A})$ & 1904 & 11766 & 3514 & 60 \\
\hline $\mathrm{H}(21 \mathrm{~A})$ & 2892 & 11391 & 435 & 74 \\
\hline $\mathrm{H}(22 \mathrm{~A})$ & 2643 & 10150 & -319 & 78 \\
\hline $\mathrm{H}(23 \mathrm{~A})$ & 2859 & 8374 & 217 & 62 \\
\hline $\mathrm{H}(26 \mathrm{~A})$ & 1911 & 12116 & 1880 & 94 \\
\hline $\mathrm{H}(26 \mathrm{~B})$ & 3267 & 12488 & 1186 & 94 \\
\hline $\mathrm{H}(26 \mathrm{C})$ & 2956 & 12606 & 2167 & 94 \\
\hline $\mathrm{H}(27 \mathrm{~A})$ & 5249 & 10520 & 1968 & 96 \\
\hline $\mathrm{H}(27 \mathrm{~B})$ & 4985 & 11641 & 2221 & 96 \\
\hline $\mathrm{H}(27 \mathrm{C})$ & 5281 & 11532 & 1238 & 96 \\
\hline $\mathrm{H}(29 \mathrm{~A})$ & 5653 & 8359 & 1448 & 74 \\
\hline $\mathrm{H}(30 \mathrm{~A})$ & 7760 & 7846 & 1349 & 82 \\
\hline $\mathrm{H}(31 \mathrm{~A})$ & 8320 & 6123 & 1618 & 73 \\
\hline $\mathrm{H}(32 \mathrm{~A})$ & 6735 & 4848 & 2075 & 72 \\
\hline$H(33 A)$ & 4625 & 5343 & 2184 & 60 \\
\hline $\mathrm{H}(35 \mathrm{~A})$ & 4513 & 7011 & 102 & 62 \\
\hline $\mathrm{H}(36 \mathrm{~A})$ & 4301 & 5884 & -760 & 76 \\
\hline $\mathrm{H}(37 \mathrm{~A})$ & 2931 & 4492 & -180 & 81 \\
\hline
\end{tabular}




$\begin{array}{lrrrr}\mathrm{H}(38 \mathrm{~A}) & 1602 & 4258 & 1263 & 72 \\ \mathrm{H}(39 \mathrm{~A}) & 1730 & 5422 & 2106 & 62 \\ \mathrm{H}(40 \mathrm{~A}) & -7104 & 2593 & 4378 & 126 \\ \mathrm{H}(40 \mathrm{~B}) & -6836 & 3608 & 4677 & 126 \\ \mathrm{H}(41 \mathrm{~A}) & -652 & 9137 & 2728 & 105 \\ \mathrm{H}(41 \mathrm{~B}) & 134 & 9816 & 1817 & 105 \\ \end{array}$

